\title{
Sex differences in spatial ability
}

Catherine Mary Jones

A thesis submitted to the University of Edinburgh in application for the degree of Doctor of Philosophy

February 2003 


\section{Declaration}

The work contained within this thesis is my own and has not been done in collaboration, except where otherwise stated. The text does not exceed 70,000 words. No part of this thesis has been submitted to any other university in application for a higher degree. 


\section{Abstract}

Males outperform females on spatial tasks in a number of mammalian species. However, sex differences are not consistently found in laboratory rats and I investigated possible reasons for this. I found no significant effect of the female oestrous cycle, landmark cues, and different types of spatial task on sex differences in spatial ability. I then hypothesised that the performance of male and female rats on spatial tasks could be due to sex differences in response to stress or food restriction. I tested groups of male and female rats in the radial arm maze under high and low stress conditions and high and low food restriction. The results were not clear-cut, but female, and not male, rats' spatial performance may have been adversely affected by high levels of food restriction. If food restricted female rats perform worse than food restricted males on spatial tasks, this could result in sex differences on appetitively-motivated spatial tasks.

In a separate experiment I predicted that male Mongolian gerbils (Meriones unguiculatus) would have better spatial ability than conspecific females because they have larger ranges and larger hippocampal volumes. Both range size and hippocampal volume is positively correlated with spatial ability. I did not find a sex difference in spatial ability in gerbils. There were also no sex differences in hippocampal volume in these subjects.

Women perform better than men on certain 'object-location' spatial tasks. This difference between the sexes is unique because men perform better than women on all other spatial tasks. I developed a test that split the object-location task into two parts, one of which involved only location memory, the other only memory for visual features. I found that women were better able to remember features than men, while the reverse was true for location memory. Therefore, women's superior performance on the object-location task may be due to their better memory for features, rather than any spatial advantage.

To conclude, I found that high levels of food restriction adversely affect female rats' spatial performance and could cause sex differences in spatial ability on appetitively- 
motivated tasks. I did not find any sex differences in either hippocampal morphology or spatial ability in gerbils. I found evidence to suggest that women's superior performance on object-location tasks is due to better feature memory rather than spatial memory, as was previously thought. 


\section{Acknowledgements}

I would like to thank the following:

The Biotechnology and Biological Sciences Research Council for funding this research;

My supervisor, Sue Healy, for giving me the chance to do this project and for always having time to speak to me;

My second supervisor, Victoria Braithwaite, for generous practical advice and equipment lending, and for her contribution to chapter 1 ;

The staff of the March animal house, especially John Tweedy and Sheena for looking after my animals and giving me practical advice on rodent husbandry;

Neil Watt and John Verth for advice on Home Office rules and regulations;

Steve Martin (ch.2 \& 3), Celine Caquineau (ch.3), Maureen Cameron (ch.3), Yvonne Harcus (ch.5), Jane Knox (ch.6), John Deag (ch.6), Lloyd Mitchell (throughout), the Darwin Workshop (especially Graham, ch.2, 4 \& 5), Graham Stone (ch.6) and Alex Hayward (ch.6) for a wide variety of essential practical assistance;

The undergraduates who allowed me to use the data they collected as part of their $4^{\text {th }}$ year projects, namely Toby Beveridge (ch.4), Stephanie Gerbert (ch.6), and Catriona Greig, Mikey Hartley, Paul Wright, Moheet Verma and Olivia Ashman (all ch.7);

All of the volunteers who provided data for chapter 7;

The Association for the Study of Animal Behaviour for providing money so that I could to attend conferences;

Edinburgh behaviour lab group for useful suggestions and comments on my data;

Robert Biegler, who wrote the program that I modified to use in chapter 7, and for useful comments on chapters 1 and 7; 
Sara Shettleworth and Josephine Pemberton for helpful comments on early versions of chapter 1;

Andy Koppe, who helped immensely with the computer program in chapter 7 , and who patiently read chapters and supported me throughout;

My family, especially my parents, Sue and Mike Jones; brother, William Jones; and grandma, Betty Leigh, for their love and support;

My office-mates (Kate, Lucy, Phil, Jono, Kirk, Zoë, Beccy, Helen, Carolyn and Marian), the other annexe inhabitants, and my various flat-mates also deserve thanks for putting up with me and keeping me entertained over the years.

A version of section 1.2 (evolutionary theories) has been published: Jones, C.M., Braithwaite, V.A. \& Healy, S.D. (2003) The evolution of sex differences in spatial ability. Behavioral Neuroscience, 117(3) 403-411.

All of the animals used in this project were treated in accordance with UK Home Office guidelines. Work in chapters $2,3 \& 6$ was carried out under Home Office project licence number 60/2786. All animals were sacrificed by an overdose of carbon dioxide gas after the experiments were completed; other groups in the department subsequently used some of the animals' organs in an attempt to reduce the overall number of animals used. 


\section{Contents}

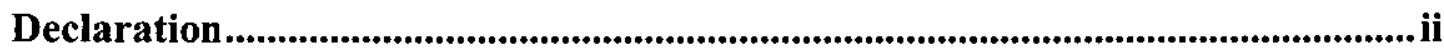

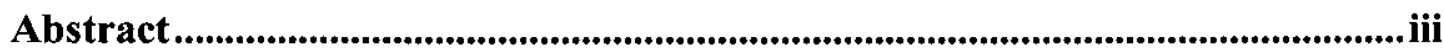

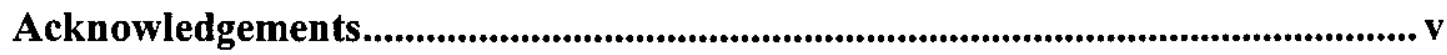

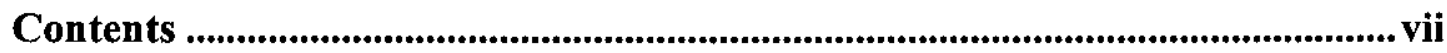

Chapter 1: Introduction and literature review ................................................ 1

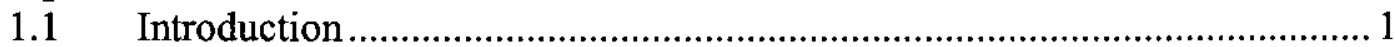

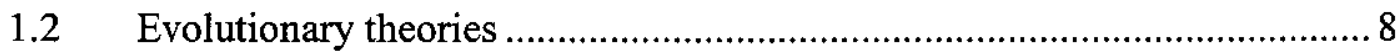

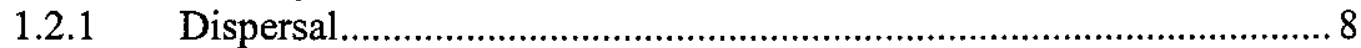

1.2.2 Fertility and parental care........................................................ 10

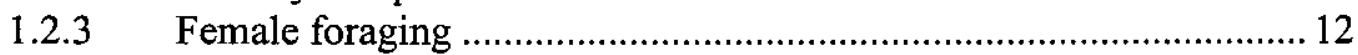

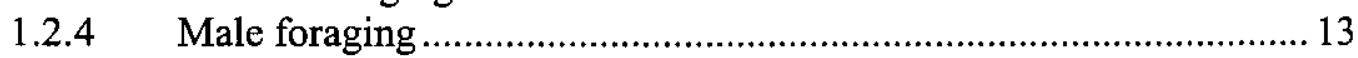

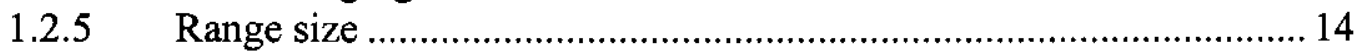

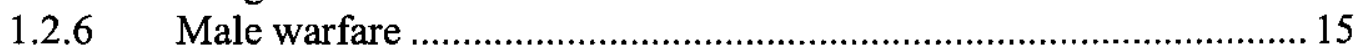

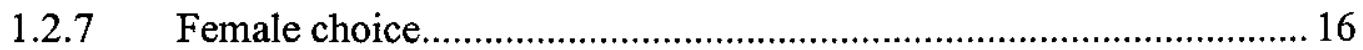

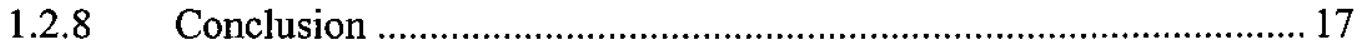

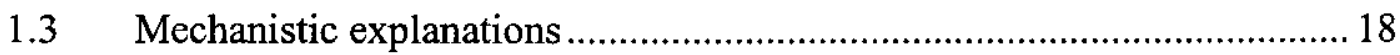

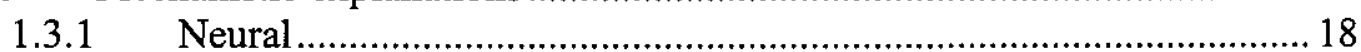

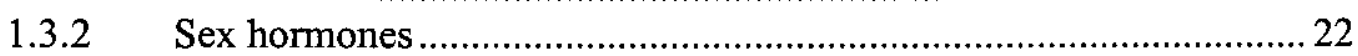

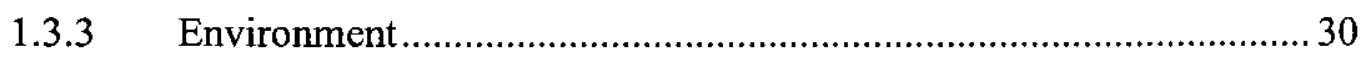

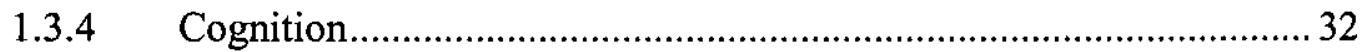

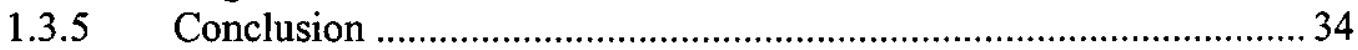

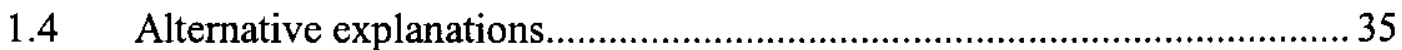

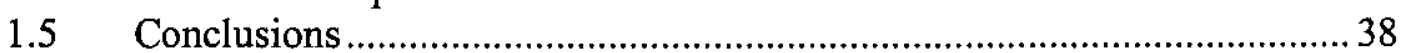

Chapter 2: Are there differences in the ways that male and female laboratory

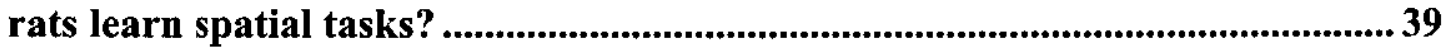

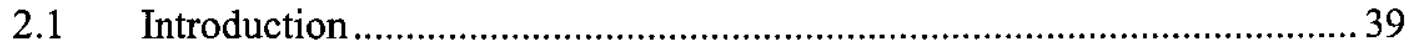

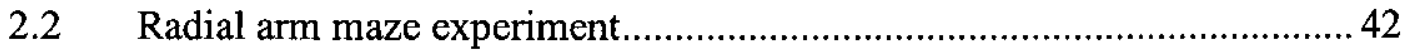

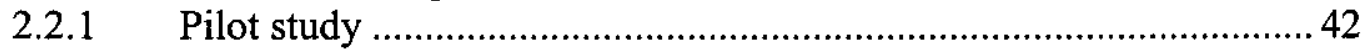

2.2.2 Materials and methods ............................................................ 42

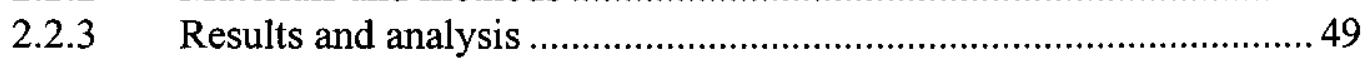

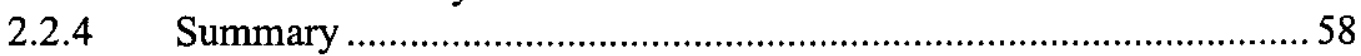

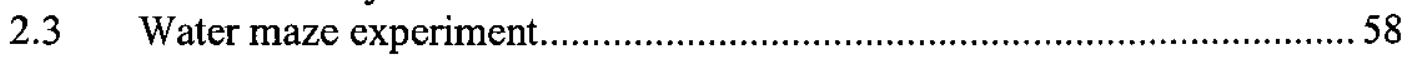

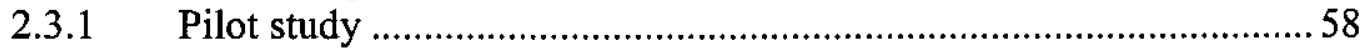

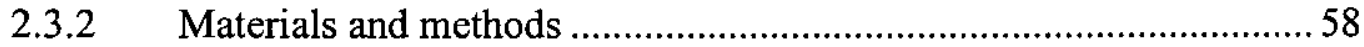

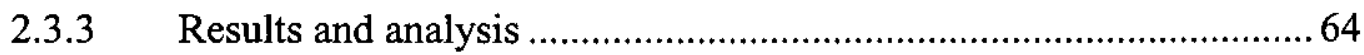

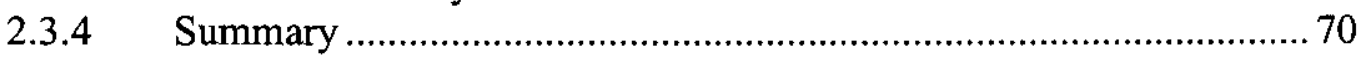

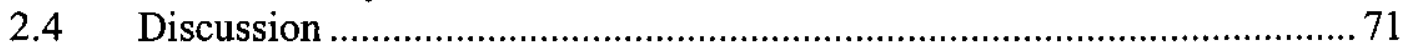

Chapter 3: Does oestrous cycle stage affect the performance of female laboratory rats on a reference memory water maze task?................................... 77

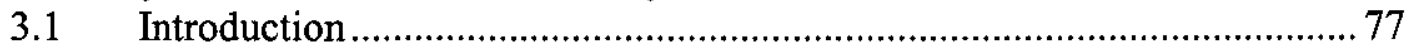

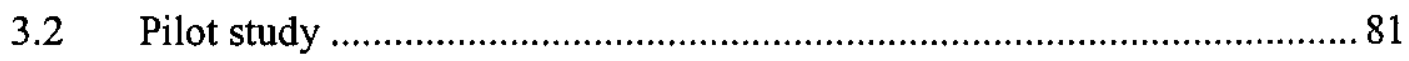




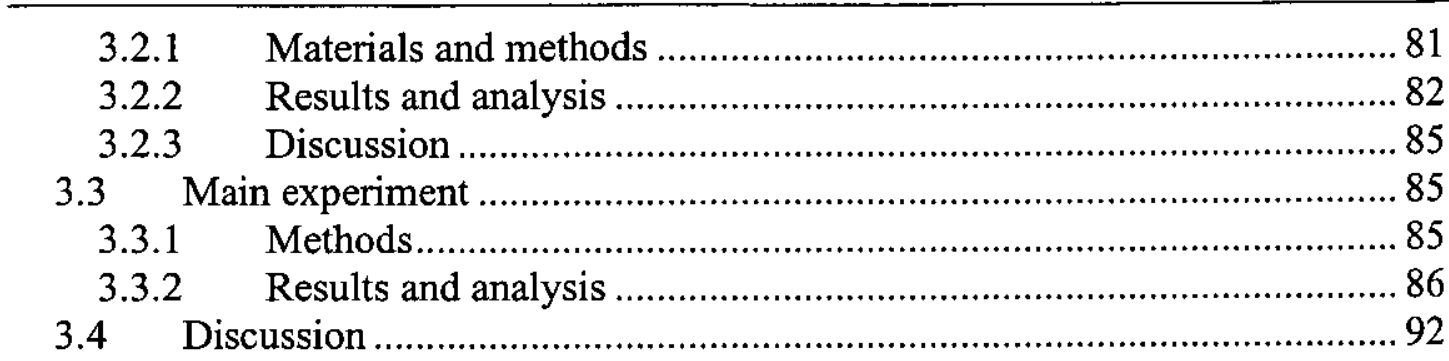

Chapter 4: The effects of acute restraint stress on the performance of male and female laboratory rats in the radial arm maze ............................................95

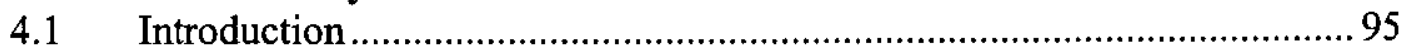

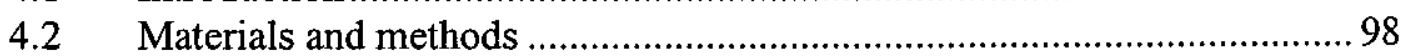

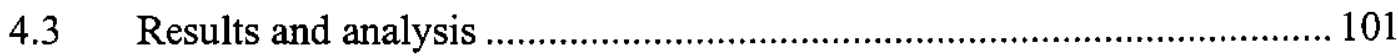

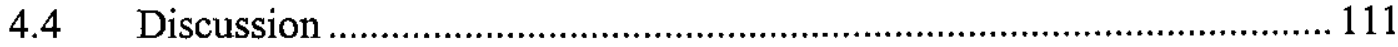

Chapter 5: The effects of different levels of food restriction on the performance of male and female laboratory rats in the radial arm maze........ 114

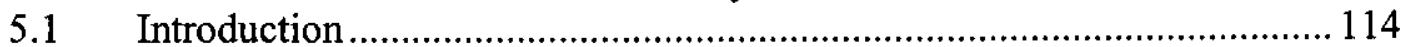

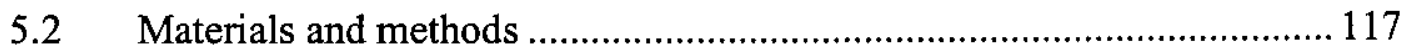

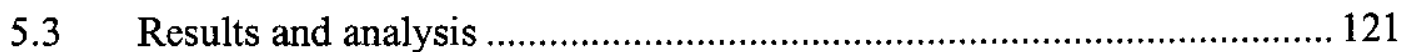

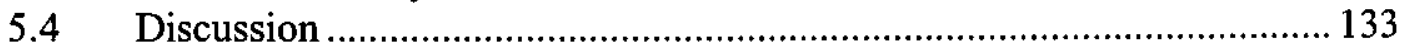

Chapter 6: Sex differences in spatial ability and hippocampal volume in Mongolian gerbils (Meriones unguiculatus)?...................................................... 138

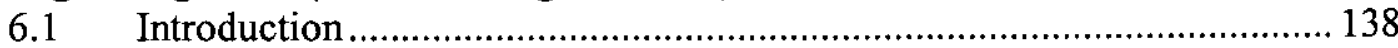

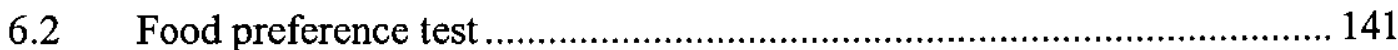

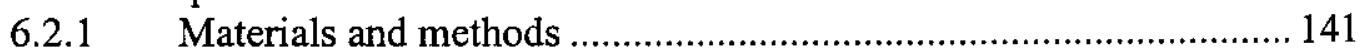

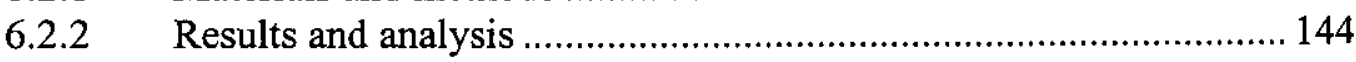

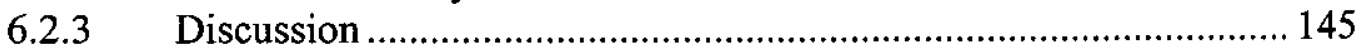

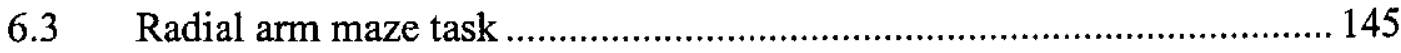

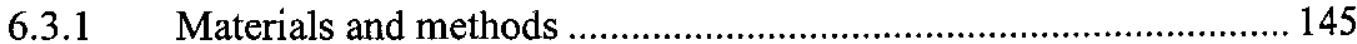

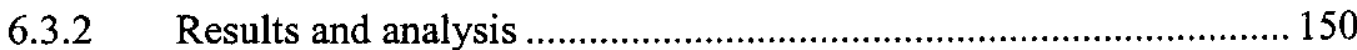

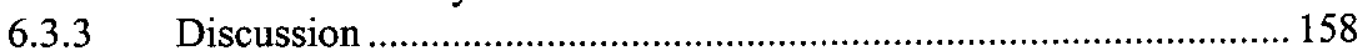

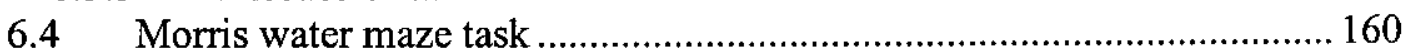

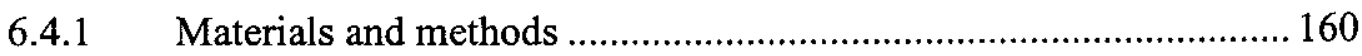

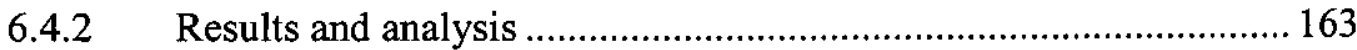

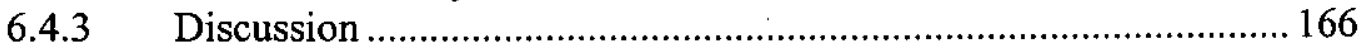

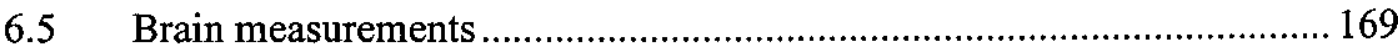

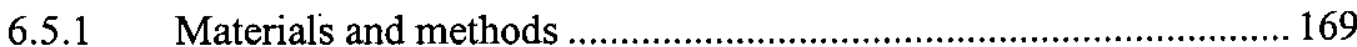

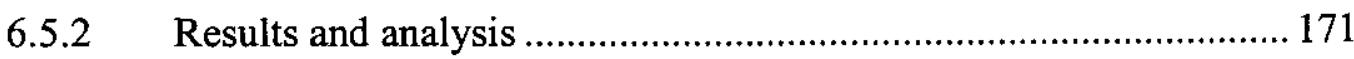

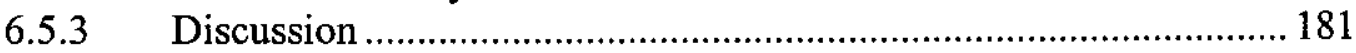

6.6 General discussion and future work .............................................. 183

Chapter 7: Sex differences in spatial and object memory in humans, and the 'hunter-gatherer' hypothesis. ................................................................................ 187

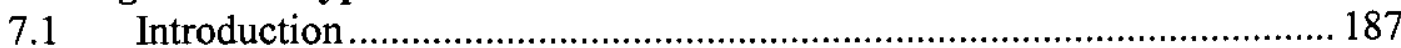

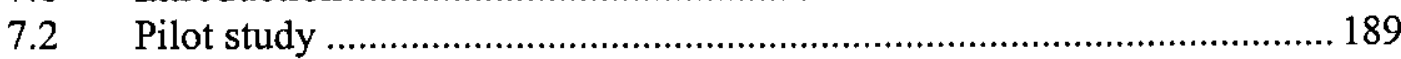

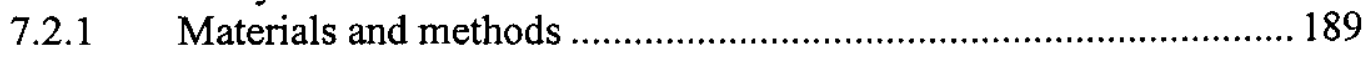

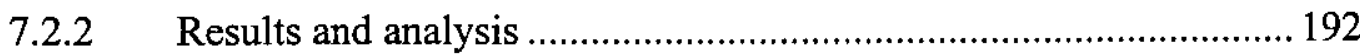




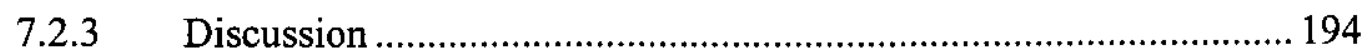

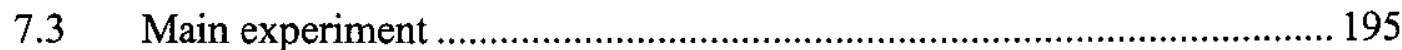

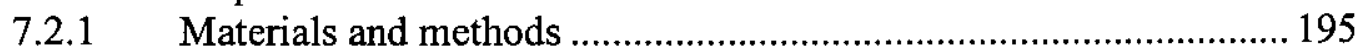

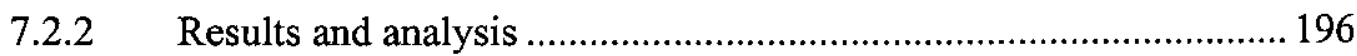

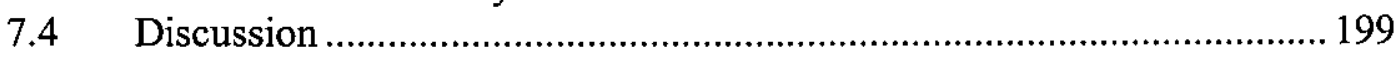

Chapter 8: General discussion..................................................................... 203

8.1 Sex differences in spatial ability in laboratory rats .................................203

8.2 Sex differences in spatial ability in Mongolian gerbils........................... 205

8.3 Sex differences in spatial ability in humans..........................................206

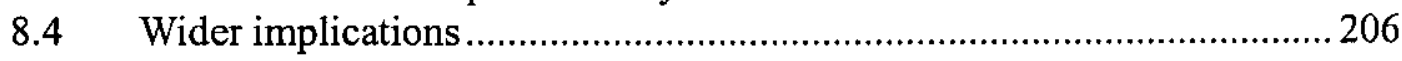

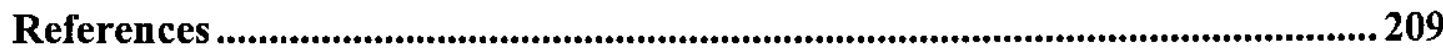




\section{Chapter 1: Introduction and literature review}

\section{$1.1 \quad$ Introduction}

Many species show morphological and physiological differences between the sexes and there is also evidence for sex differences in cognitive abilities (e.g. Halpern, 1991; Kimura, 1999; Mealey, 2000). One of the best-studied cognitive sex differences is an apparent male advantage in spatial cognition. There are a number of evolutionary hypotheses that have been proposed to explain this difference, and there are also endocrinological and neurophysiological data providing the mechanistic basis for such a difference between the sexes.

\section{What is spatial ability?}

Spatial ability is not a unitary ability; rather the concept is likely to encompass several different cognitive abilities all of which are involved in navigation and the perception of three-dimensional objects. In humans spatial ability has been defined as "the ability to imagine what an irregular figure would look like if it were rotated in space or the ability to discern the relationship among shapes and objects" (Halpern, 1991). However, Linn and Peterson's (1985) meta-analysis of studies on sex differences in spatial abilities found that the studies tested three distinct types of spatial abilities: spatial perception, the ability to ignore distracting information in order to determine spatial relations; mental rotation, the ability to use the imagination to quickly and accurately rotate figures; and spatial visualization, being able to manipulate complex spatial information through several stages to reach the correct solution.

In both rodents and humans many different tests of spatial ability are used (figures $1.1 \& 1.2)$ and different types of test may measure different cognitive abilities. Spatial ability tests in rodents usually involve the subjects learning to complete a maze. However, even within maze tests different protocols may measure different 
things. For example, the memory involved may be either working or reference memory. Working memory can be defined as retaining trial-specific information, which is usually only useful for a short period of time, while reference memory involves information that remains constant over time (task-specific information) and is usually of a longer duration (Bimonte et al., 2000).

Maze tasks can be solved using many different strategies; for example, distal cues such as distant landmarks or room geometry, local landmarks, cues relative to the subject's body, or path integration (a form of dead reckoning where a compass and odometer update an accumulator to record current position). Such strategies can be classified as either egocentric or allocentric (O'Keefe \& Nadel, 1978; Benhamou, 1997). Egocentric strategies use the body as a frame of reference, for example response chains (i.e. remembering a list of left and right turns). Allocentric (also called exocentric) strategies, on the other hand, are relative to the environment or objects within the environment. Examples of allocentric strategies include the use of landmarks, gradient maps and compass directions.

Which strategy is used may depend on the maze and the task: a Davenport maze may be solved by a response chain; a radial arm maze (RAM) with all arms rewarded (working memory task) may be effectively solved using an adjacent arm strategy; but in order to solve a partially rewarded RAM (working and reference memory task) efficiently and avoid unrewarded arms, cues either within or outside the maze must be used to distinguish individual arms; the Morris water maze (MWM) has been designed to exclude intra-maze cues and so can only be solved using cues outside the maze (Hodges, 1996). Different strategies may also be used to solve the same maze, for example a MWM may be solved using either landmarks outside the maze or geometrical cues. There is evidence that the cues used may depend on sex (discussed in detail in section 1.3.4).

Therefore, when assessing the spatial ability literature it is important to consider the type of test used. Different tests may measure different forms of spatial ability, which may in turn show different magnitudes of sex differences. The working definition of spatial ability that I will use, unless otherwise stated, is that it is the 
ability used by an animal to navigate around its environment, as this is a demand common to all animal species.

Figure 1.1: Examples of tests used to assess spatial ability in humans.

a) Mental rotation test. Which of the figures on the right are the same as the one on the left, except for orientation?
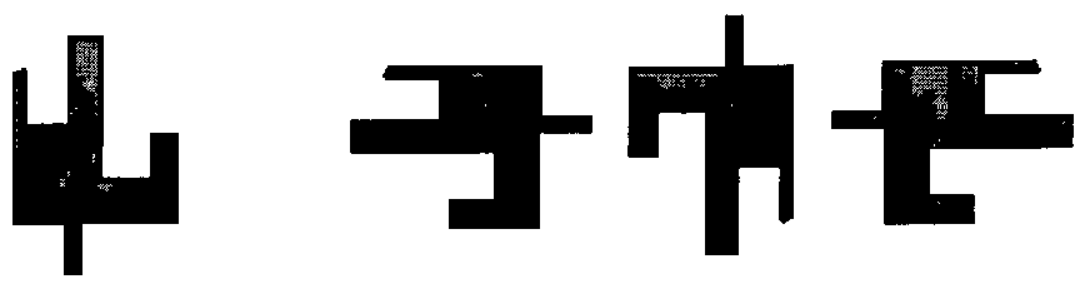

b) Embedded figures test. Trace the outline of the shape on the left within the figure on the right.
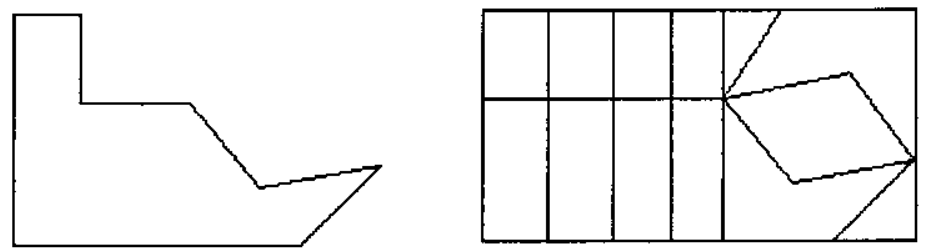
Figure 1.2: Maze tasks used to study sex differences in spatial ability in rodents.

a) Morris water maze

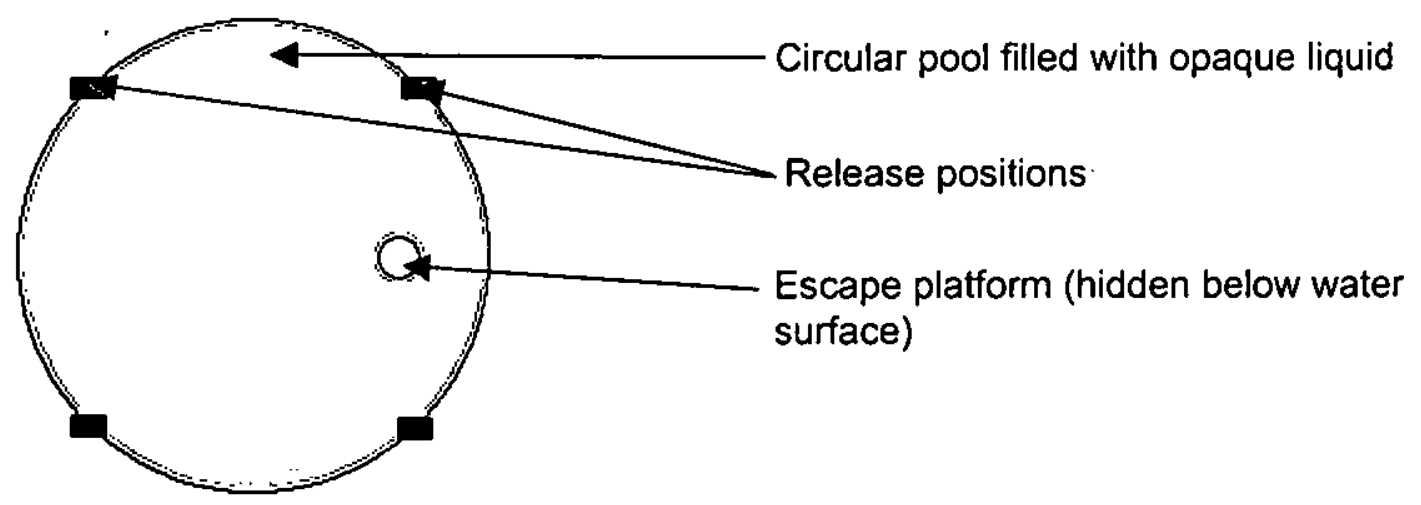

b) Radial arm maze (8-arm maze fully rewarded for a working memory task)

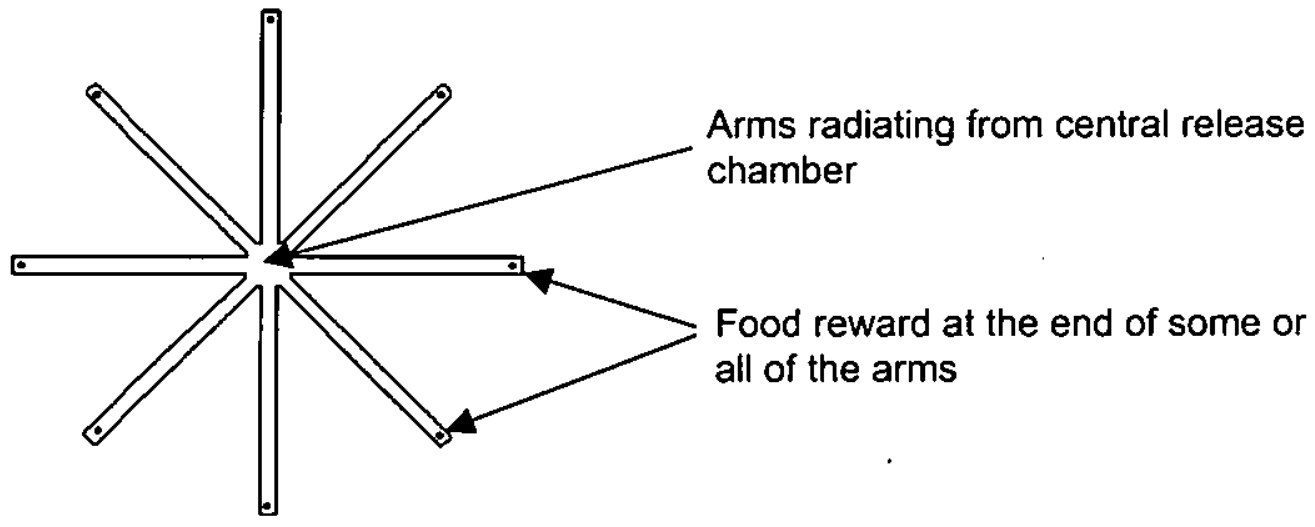

c) Davenport maze

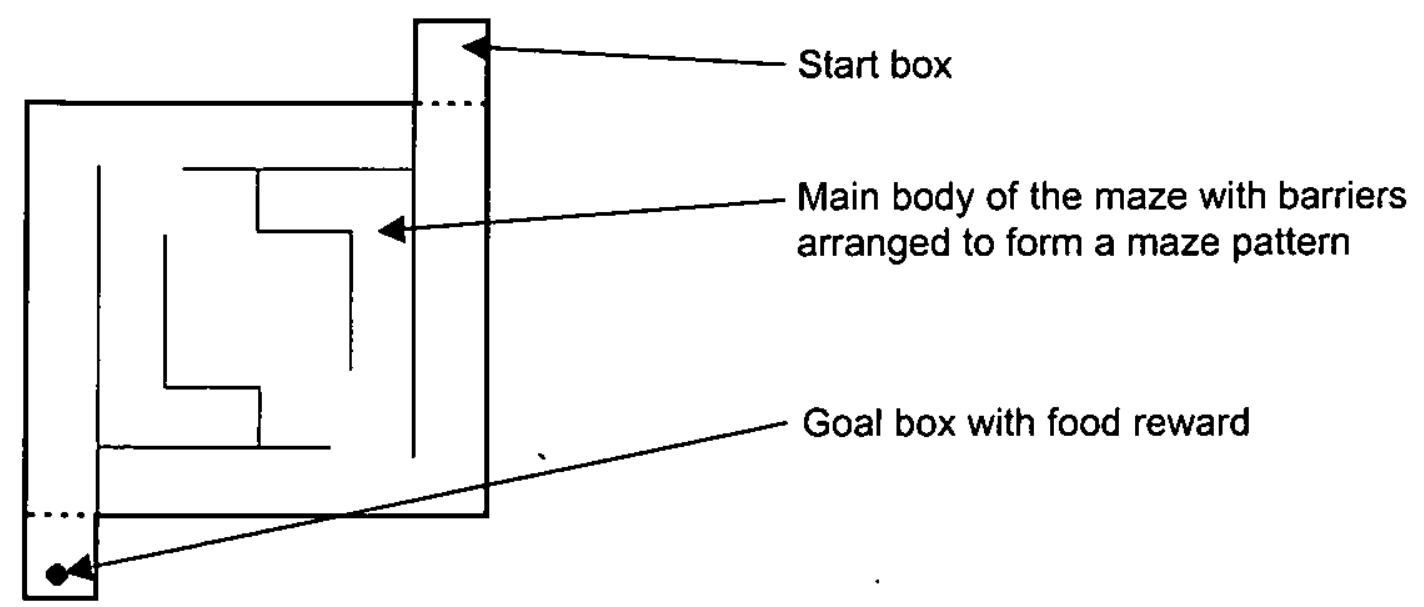




\section{Which species show sex differences in spatial ability?}

Although the male advantage in spatial cognition is sometimes considered to be universal, to my knowledge only 11 species have actually been tested for sex differences in spatial ability (table 1.1). Of these, only six species, all of which are primates or rodents, show a male advantage in spatial ability. Five other species (more rodents and a single bird species) have been tested but no differences have been found.

Laboratory rats were the first animals in which males were shown to have better spatial ability than females (e.g. Corey, 1930), and they are still the species of choice for most of the work in this area. However, they are also the species in which sex differences in spatial ability are least consistently found. Some studies find superior male performance on spatial tasks and some do not for no obvious reason, even within the same laboratory (e.g. Kanit et al., 1998a \& b; table 1.2).

There is variation, both within and between species, in sex differences in spatial ability that needs to be explained.

\section{What causes sex differences in spatial ability?}

There are several levels at which sex differences in spatial ability may be explained. First, there is the evolutionary level, under the auspices of which many hypotheses have been proposed, but few rigorous tests have been carried out. Second, sex differences in spatial ability may be explained mechanistically, and a considerable number of experiments have been carried out to investigate such possibilities. Third, the observed sex differences in spatial ability may simply be a by-product of selection for another feature. They may exist, but be non-adaptive; or not exist under natural conditions. They may be observed only because of the way that spatial ability is tested in the laboratory. This third possibility is discussed with particular reference to laboratory rats because of the inconsistent findings of sex differences in spatial ability in these animals. 
Table 1.1: Species that have been tested for sex differences in spatial ability.

\begin{tabular}{|c|c|c|}
\hline Species & Sex differences? & Reference \\
\hline $\begin{array}{l}\text { Shiny cowbird } \\
\text { (Molothrus } \\
\text { bonariensis) }\end{array}$ & No & Astie et al., 1998 \\
\hline $\begin{array}{l}\text { Rhesus monkey } \\
\text { (Macaca mulatta) }\end{array}$ & Male>female & Lacreuse et al., 1999 \\
\hline Human & $\begin{array}{l}\text { Male>female (except for } \\
\text { object-location tasks where } \\
\text { female>male) }\end{array}$ & $\begin{array}{l}\text { E.g. Dabbs et al., 1998; } \\
\text { Eals \& Silverman, 1994 }\end{array}$ \\
\hline $\begin{array}{l}\text { Guinea pig (Cavia } \\
\text { porcellus) }\end{array}$ & No & Dringenberg et al., 2001 \\
\hline $\begin{array}{l}\text { Kangaroo rat } \\
\text { (Dipdomys deserti) }\end{array}$ & No & Langley, 1994 \\
\hline $\begin{array}{l}\text { Deer mouse } \\
\text { (Peromyscus } \\
\text { maniculatus) }\end{array}$ & Male>female & Galea et al., 1994a \\
\hline $\begin{array}{l}\text { Prairie vole (Microtus } \\
\text { ochrogaster) }\end{array}$ & No & Gaulin \& Fitzgerald, 1989 \\
\hline Laboratory mouse & $\begin{array}{l}\text { Depends on strain, when } \\
\text { found male>female }\end{array}$ & $\begin{array}{l}\text { Mishima et al., 1986; } \\
\text { Berger-Sweeney et al., } \\
\text { 1995; Frick et al., } 2000\end{array}$ \\
\hline $\begin{array}{l}\text { Meadow vole } \\
\text { (Microtus } \\
\text { pennsylvanicus) }\end{array}$ & Male>female & Gaulin \& Fitzgerald, 1986 \\
\hline $\begin{array}{l}\text { Pine vole (Microtus } \\
\text { pinetorum) }\end{array}$ & No & Gaulin \& Fitzgerald, 1986 \\
\hline Laboratory rat & $\begin{array}{l}\text { Depends on study, but when } \\
\text { found male>female }\end{array}$ & See table 1.2 \\
\hline
\end{tabular}


Table 1.2: Examples of studies that have tested for sex differences in spatial ability in laboratory rats. All results are from comparisons of control (un-manipulated) males and females. $M W M=$ Morris water maze, $\mathrm{RAM}=$ radial arm maze.

\begin{tabular}{|c|c|c|c|}
\hline Study & Maze type & $\begin{array}{l}\text { Working or reference } \\
\text { memory task? }\end{array}$ & $\begin{array}{l}\text { Were there sex } \\
\text { differences? }\end{array}$ \\
\hline $\begin{array}{l}\text { Cimadevilla et al., } \\
1999\end{array}$ & MWM & Reference & Yes \\
\hline Markowska, 1999 & MWM & Reference & No \\
\hline Kanit et al., 1998a & MWM & Reference & No \\
\hline Kanit et al., 1998b & MWM & Reference & Yes \\
\hline $\begin{array}{l}\text { Isgor \& Sengelaub, } \\
1998\end{array}$ & MWM & Reference & Yes \\
\hline $\begin{array}{l}\text { Warren \& Juraska, } \\
1997\end{array}$ & MWM & Working & No \\
\hline Bucci et al., 1995 & MWM & Reference & No \\
\hline Roof et al., 1993 & MWM & Working & No \\
\hline $\begin{array}{l}\text { Roof \& Havens, } \\
1992\end{array}$ & MWM & Reference & Yes \\
\hline $\begin{array}{l}\text { Seymoure et al., } \\
1996\end{array}$ & RAM & $\begin{array}{l}\text { Working \& reference (11/17 } \\
\text { arms rewarded) }\end{array}$ & Yes \\
\hline $\begin{array}{l}\text { Luine \& Rodriguez, } \\
1994\end{array}$ & RAM & $\begin{array}{l}\text { Working (all } 8 \text { arms } \\
\text { rewarded) }\end{array}$ & Yes \\
\hline $\begin{array}{l}\text { Maier \& Pohorecky, } \\
1986\end{array}$ & RAM & $\begin{array}{l}\text { Working \& reference ( } 4 / 8 \\
\text { arms rewarded) }\end{array}$ & No \\
\hline Juraska et al., 1984 & RAM & $\begin{array}{l}\text { Working (all } 17 \text { arms } \\
\text { rewarded) }\end{array}$ & No \\
\hline Einon, 1980 & RAM & $\begin{array}{l}\text { Working (all } 8 \text { arms } \\
\text { rewarded) }\end{array}$ & Yes \\
\hline Joseph, 1979 & $\begin{array}{l}\text { Davenport } \\
\text { maze }\end{array}$ & Reference & Yes \\
\hline Joseph et al., 1978 & $\begin{array}{l}\text { Davenport } \\
\text { maze }\end{array}$ & Reference & Yes \\
\hline $\begin{array}{l}\text { Davenport et al., } \\
1970\end{array}$ & $\begin{array}{l}\text { Davenport } \\
\text { maze }\end{array}$ & Reference & Yes \\
\hline
\end{tabular}




\subsection{Evolutionary theories}

Two different selection processes can result in the evolution of differences between males and females of the same species. The first is natural selection, which may occur within species if the males and females occupy different niches and so are exposed to different selection pressures. For example, males and females of the same species may exploit different foods or be exposed to different predation regimes. The second adaptive process is sexual selection. Sexual selection theory has been proposed specifically to explain obvious sexual dimorphisms such as the peacock's tail and stag's antlers. This theory states that a character can evolve if it gives the individual possessing it an advantage in attracting mates, even if the character is disadvantageous for survival. Such selection is intra-sexual if the character results from direct competition between members of the same sex for mates, or inter-sexual if the character is selected because it makes the individual displaying it preferred as a mate by members of the opposite sex.

At least seven different evolutionary theories have been proposed to explain sex differences in spatial ability. Some involve natural and some sexual selection. Many of them have been proposed with reference only to humans but most of these can be extended to apply to any species. Many of the theories have undergone little rigorous testing. I shall summarize each in turn, paying particular attention to supporting data and any weaknesses.

\subsubsection{Dispersal}

The dispersal hypothesis states that in species with sex-biased juvenile dispersal the further dispersing sex will show superior spatial ability (Silverman \& Eals, 1992). There are few species for which data about sex differences in both dispersal and spatial ability are available, but what data there are offer ambivalent support.

Women tend to disperse further than men in most human societies (e.g. Koenig, 1989), so in humans this hypothesis would predict greater spatial ability in women. The data from tests of spatial ability in humans using real navigation tasks such as 
way-finding do not support this prediction (e.g. Moffat et al., 1998; Silverman et al., 2000).

In meadow voles and prairie voles, males disperse further than females (Boonstra et al., 1987). Therefore the prediction is that there should be sex differences in spatial ability favouring males in both of these species. This is supported by the data from meadow voles as males perform better on a symmetrical maze task than females. However, contrary to the prediction, there are no sex differences in prairie voles tested on the same task (Gaulin \& Fitzgerald, 1989).

A correlation between dispersal and spatial ability can be found in rhesus monkeys and deer mice. Male rhesus monkeys are the dispersing sex and they appear to have superior spatial abilities to females (Drickamer \& Vessey, 1973; Kaplan et al., 1995; Lacreuse et al, 1999). Male deer mice also tend to disperse further than females and perform better on spatial tasks (Dice \& Howard, 1951; King, 1983; Galea et al., 1994a).

Data from three out of five species appear to lend support to the dispersal hypothesis. However, tasks used to test spatial ability typically test short-range, frequent-use spatial ability, rather than mimicking the one-off long-range spatial ability that is presumably needed for dispersal.

In the avian literature there is a wealth of evidence suggesting that movements over long and short distances utilise different cognitive abilities. First, behavioural studies have shown that birds use different cues for long- and short-range journeys. Birds on long distance journeys tend to use information from stellar and magnetic compasses, and use landmarks such as mountain ranges in a compass-like way to indicate direction rather than as a map (Bruderer, 1982; Papi \& Wallraff, 1992). Over short, familiar distances birds appear to make navigation decisions using local landmarks (Braithwaite \& Guilford, 1991). The second line of evidence comes from the hippocampus, which is a region of the brain that is heavily implicated in the acquisition and use of spatial information, both in birds and in mammals (e.g. Morris et al., 1982; Sherry et al., 1992). Strasser et al. (1998) found that pigeons with 
hippocampal lesions had disrupted short, but not long-range navigation. This again supports the theory that travelling long and short distances requires different cognitive processes.

These various avian experiments suggest that dispersal and day-to-day spatial tasks may involve two separate abilities and that dispersal is therefore unlikely to have acted as a strong selective force on the type of spatial memory measured in conventional tests. Sex differences in dispersal are unlikely to be the cause of the observed sex differences in spatial ability.

\subsubsection{Fertility and parental care}

The fertility and parental care hypothesis (Sherry \& Hampson, 1997) states that female reproductive success is enhanced by reduced mobility during reproductive periods, in order to save energy for reproduction and reduce the risk of accidents and predation. This hypothesis is then used to predict that spatial ability will be reduced in females during reproductive periods.

It is unclear exactly what constitutes a 'reproductive period'. Sherry and Hampson (1997) state that it is not simply the stage of a female's fertility cycle when she is most likely to conceive. Rather it seems to be the period during which the female is actually reproducing. In mammals there is some evidence that late pregnancy is correlated with reduced mobility. Mice in residential mazes are less active during late pregnancy (Barnett \& McEwan, 1973), and pregnant women spend more time doing sedentary activities and resting than non-pregnant women (English \& Hitchcock, 1968; Durnin, 1991; Prentice et al., 1996). Late pregnancy therefore seems to be the time at which this hypothesis predicts female mammals to have reduced spatial ability.

A study that tested women's spatial ability when they were pregnant and again following childbirth provides data that are consistent with the predictions of this hypothesis (Woodfield, 1984; using the embedded figures test, figure 1.1.b). The women were tested in the $38^{\text {th }}-40^{\text {th }}$ week of pregnancy and again while they were breastfeeding six weeks after giving birth. They performed better on the test while 
they were breastfeeding than when they were pregnant, an improvement that was greater than that of a control group of non-pregnant women tested at intervals of similar duration. The women had reduced spatial ability during the late stages of pregnancy, as predicted by the fertility and parental care hypothesis.

Galea et al. (2000) found that pregnant rats in the third trimester travelled further to reach the hidden platform in a MWM than non-pregnant animals, suggesting some impairment of their spatial ability. However, pregnant rats in the first and second trimesters tended to be better at the test than non-pregnant females. There were no differences in swim speed between the groups. However, it should be noted that swim speed does not automatically correlate with activity measured on dry land, and neither of these are necessarily related to mobility (Lehmann et al., 2002). These data are, then, equivocal in their support for the hypothesis: the reduced spatial ability during late pregnancy is in accordance with the hypothesis, but the lack of a reduction in swim speed is not.

Data from spatial tests of deer mice also support the hypothesis. Female deer mice in the breeding season perform more poorly on a water maze task than do males (although it is not clear in this study whether the females were pregnant or caring for young; Galea et al., 1994a). Outside the breeding season there is no sex difference in water maze performance. Swim speed did not differ between the sexes during the breeding season. However, this result is also consistent with the range size hypothesis (section 1.2.5).

The fertility and parental care hypothesis is the only evolutionary hypothesis that can explain the observed negative correlation between oestrogen levels and spatial ability (section 1.3.2.2), as it is the only hypothesis that predicts that sex differences in spatial ability have evolved through selection to reduce female spatial ability. However, testosterone levels are also correlated with spatial ability, suggesting that selection has also acted on male spatial ability. Therefore the fertility and parental care hypothesis is unlikely to be the sole explanation for the observed sex differences in spatial ability. 
There is some experimental evidence in support of this hypothesis, however it must be remembered that the assumption on which this hypothesis is based, namely that females move less during reproductive periods, has not been tested. Even if females are shown to have reduced mobility during reproductive periods, decreased spatial ability is only one possible cause of this effect. Other possible mechanisms such as having dependent young, or reduced internal motivation to move need to be investigated.

\subsubsection{Female foraging}

Silverman and Eals (1992) proposed the female foraging hypothesis specifically in relation to humans. It forms a pair with the male foraging hypothesis in that both are based on the assumption that humans have evolved a division of foraging labour between the sexes. Men evolved to hunt for food, while women are adapted for gathering it (Hawkes, 1991). Successful female foraging would therefore involve remembering the location of food plants within complex arrays of vegetation over timescales ranging from a few days to a year. This leads to the prediction that women will show an advantage over men on specific spatial tasks that involve remembering the locations of objects within a complex array, so called objectlocation tasks. This hypothesis is the only one to predict superior female spatial ability.

Object-location tasks typically involve the subject being allowed to view an array of objects for a certain amount of time. The objects are then hidden and the subject has to remember their locations. For example, in Silverman and Eals' (1992) original study, the subjects were given a piece of paper showing pictures of 27 objects and allowed to view it for $1 \mathrm{~min}$. The paper was then put away and they were given a new sheet showing the same pictures as before, but with some of them moved. The subjects were asked to identify which objects had moved location. In accordance with the female foraging hypothesis women are typically better than men at object location tasks, both on paper and in three dimensions (Silverman \& Eals, 1992; Eals \& Silverman, 1994; James \& Kimura, 1997; McBurney et al., 1997; Montello et al., 
1999; Duff \& Hampson, 2001), although there are some exceptions (Dabbs et al., 1998; Postma et al., 1998; 1999; Duff \& Hampson, 2001).

Unfortunately the female foraging hypothesis has been proposed exclusively in relation to humans and is difficult to generalise to other species.

\subsubsection{Male foraging}

This hypothesis is complementary to the female foraging hypothesis. It is again based on division of foraging labour between men and women. It states that men will be better than women at tasks involved in hunting (Lovejoy, 1981).

There is evidence that men are more accurate at motor tasks such as throwing and intercepting a ball, although whether or not these are truly spatial remains in doubt (Watson \& Kimura, 1991).

Men are also better than women at geographic tasks such as giving directions or way-finding in woodlands (Dabbs et al., 1998; Silverman et al., 2000). The mental rotation test is a pencil-and-paper task that consistently shows large sex differences favouring men. Mental rotation scores are positively correlated with performance on navigational tasks, which suggests that these tasks are being solved in the same way (e.g. Dabbs et al., 1998; Moffat et al., 1998; Silverman et al., 2000). However, these findings are also consistent with the range size, male warfare and female choice evolutionary hypotheses (sections 1.2.5-7). The results show that men are better than women at finding their way around the environment, but they do not shed any light on the selective pressures that resulted in men being better at navigation tasks.

Again, this hypothesis has been proposed solely in relation to humans. However, unlike the female foraging hypothesis it may be possible to extend this hypothesis to other species where the male forages in order to provision his more stationary mate, such as hornbills (Bucerotidae) and wolves (Canis lupus). 


\subsubsection{Range size}

The range size hypothesis links spatial ability to range size and mating system (Gray \& Buffery, 1971). It predicts that sex differences in spatial abilities will only be found in species where males have larger home ranges than females. In such species, males will show enhanced spatial ability compared to females because they range over a larger area in order to father offspring with several females and maximize their reproductive success (Gaulin, 1995).

Data from three species of vole provide some support for this prediction: the promiscuous male meadow vole has a range that is much larger than the conspecific female range, whereas there is no difference in range size between the sexes in the monogamous prairie and pine voles. Male meadow voles outperformed conspecific females on maze tasks, but there were no sex differences in performance in either the pine or the prairie voles as predicted by the hypothesis (Gaulin \& Fitzgerald, 1986; 1989). The sex difference in meadow voles has also been observed in the water maze, which suggests that the result is robust to changes in task type (Kavaliers et al., 1998).

Tests of spatial ability in deer mice and desert kangaroo rats (Dipodomys deserti) have also produced results that are consistent with the range size hypothesis. Deer mice are polygynous with males having larger home ranges than females during the breeding season, while in the monogamous desert kangaroo rat there are no sex differences in range size. In the breeding season, male deer mice outperformed conspecific females in a MWM task but there were no sex differences among the kangaroo rats on a task where they were required to remember the location of a token buried in a sandbox (Galea et al., 1994a; Langley, 1994).

The predictions of this hypothesis are also compatible with the observed sex differences in spatial ability in rats and humans, as both species are thought to be somewhat polygynous with males tending to have larger spatial ranges than females (Taylor, 1978; Macdonald \& Fenn, 1995; Gaulin \& Hoffman, 1998). Data from mice are similarly supportive: wild male house mice have larger ranges than 
conspecific females, and male laboratory mice outperform females in a RAM (Mishima et al., 1986; Zielinski et al., 1992; Chambers et al., 2000).

Comparisons within species also provide support for the range size hypothesis. Deer mice and meadow vole males only have larger ranges than females during the breeding season (Gaulin \& Fitzgerald, 1989; Galea et al., 1994a). Male deer mice perform better than females in the water maze during the breeding season but not when tested during the non-breeding season (Galea et al., 1994a). There are also sex differences in meadow voles in breeding condition but not between non-breeding subjects (Galea et al., 1994c; Galea et al., 1995; Kavaliers et al., 1998).

The mammalian data conform better to the predictions of the range size hypothesis than to any of the others. However, it must be noted that in humans there is still little to distinguish this hypothesis from the male foraging, male warfare and female choice hypotheses which all predict the same correlation between the size of the area over which an animal ranges and spatial ability. The hypotheses vary only in the rationale as to why the sexes might differ in range size.

\subsubsection{Male warfare}

This hypothesis proposes that sex differences in human spatial ability have evolved by intra-sexual competition in the form of small-scale warfare between men (Geary, 1995; Sherry \& Hampson, 1997). The male warfare hypothesis proposes that men travel long distances in order to ambush other men (and therefore reduce competition for resources) and capture females, and therefore need superior navigational ability (Buss \& Shackelford, 1997).

Although small-scale warfare does appear to be related to male mortality and individual differences in offspring number in extant hunter-gatherer societies there is very little other support or evidence for this hypothesis (Chagnon, 1983). Men are better than women at navigational tasks, but this finding is also predicted by three other evolutionary hypotheses. I am also unsure of the relevance of this hypothesis when compared to the male foraging hypothesis, for example. Men might travel for warfare once every few months, but travel to hunt for food every few days. Warfare 
usually involves attacking acquaintances rather than total strangers, and therefore when men travel for warfare they are usually going to places that they already know through hunting or trading trips (Chagnon, 1983). Therefore, warfare seems to be unlikely to have been as strong a selection pressure on male spatial ability as hunting. This hypothesis is not only logically flawed, but also only applies to humans and is therefore of limited value in explaining the rodent data.

\subsubsection{Female choice}

This hypothesis proposes that females will prefer males who are successful hunters as mates, and that hunters are successful because of their superior spatial ability (Sherry \& Hampson, 1997). Like the range size and male warfare hypotheses, this hypothesis predicts that sex differences in spatial ability have evolved via sexual rather than natural selection, and that variation in male spatial ability will therefore correlate with mating success rather than with survival. The female choice hypothesis differs from the other two by proposing that the mechanism is intersexual selection rather than intra-sexual selection.

There have been no direct tests of this hypothesis. However, in hunter-gatherer societies such as the Ache tribe of Eastern Paraguay, men share animals that they have killed among the whole tribe, rather than just within their family group (Hawkes, 1991). This public sharing may have a display function, by conspicuously demonstrating foraging success. Whether or not the women of the tribe use this sharing to make subsequent mate choice decisions is not clear. Bird et al. (2001) observed spear fishing and turtle hunting in the Meriam people of Torres Strait, Australia, and concluded that these hunting practices fulfilled criteria to be costly signals honestly displaying quality. Again, it is unknown whether women use the signals to make mate choices, and also whether hunting success is correlated with spatial ability.

Unlike the female foraging and male warfare hypotheses, this hypothesis may plausibly be investigated in non-human animals. Courtship feeding, particularly in birds, is often considered a way in which females assess the quality of their males 
(e.g. Gonzalez-Solis et al., 2001; Bussiere, 2002), although the possibility that the male is thereby specifically demonstrating his spatial ability has not been discussed previously.

\subsubsection{Conclusion}

The seven hypotheses are summarised in table 1.3. Many evolutionary hypotheses have been proposed to explain sex differences in spatial ability, but little work has been done to test them. Of the seven theories that I have discussed the dispersal and male warfare hypotheses appear to contain logical flaws that make them unlikely to be good explanations for the evolution of sex differences in spatial ability. The female foraging hypothesis only relates to a subset of spatial tasks in a single species (humans) and is therefore of limited value as a general explanation of the evolution of sex differences in in spatial ability. The dispersal, fertility and parental care, male warfare and female choice hypotheses remain untested. There is some evidence for the male and female foraging hypotheses in humans, and for the range size hypothesis in some rodent species.

One of the main problems with the hypotheses is that four of them revolve around the same premise, namely that males travel further than females and therefore require better spatial ability to navigate. They only differ in the reason for males travelling further. The data confirm that males of some species do have better navigational abilities than females, but do not allow us to distinguish between the hypotheses.

Whichever evolutionary pressures have been acting to cause sex differences in spatial ability they are mediated by the effects that genes have on the body. Although evolutionary pressures are the ultimate cause of sex differences in spatial ability, differences between males' and females' bodies are the proximate cause. Describing the mechanism of spatial cognition and how it differs between males and females is therefore a complementary way to explain sex differences in spatial abilities. 
Table 1.3: A summary of the evolutionary hypotheses that have been proposed to explain sex differences in spatial ability.

\begin{tabular}{l|l|l|l} 
Hypothesis & Species & Prediction & Selective pressure \\
\hline $\begin{array}{l}\text { Dispersal } \\
\text { pertility and }\end{array}$ & Any & $\begin{array}{l}\text { Dispersing sex } \\
\text { better } \\
\text { Female<male }\end{array}$ & Natal dispersal distance \\
$\begin{array}{l}\text { Female foraging } \\
\text { Male foraging }\end{array}$ & Humans & Female>male & $\begin{array}{l}\text { Females reduce mobility to } \\
\text { reproductive periods } \\
\text { Division of foraging labour }- \\
\text { women remember locations of food } \\
\text { sources }\end{array}$ \\
Range size & Any & Male>female & $\begin{array}{l}\text { Division of foraging labour - men } \\
\text { use navigation skills for hunting }\end{array}$ \\
Male warfare & Humans & Male>female & $\begin{array}{l}\text { Polygynous males have larger } \\
\text { ranges to mate with more females } \\
\text { Men travel long distances to kill } \\
\text { competitors and capture women }\end{array}$ \\
Female choice & Humans & Male>female & $\begin{array}{l}\text { Women choose mates on the basis } \\
\text { of their hunting success }\end{array}$
\end{tabular}

\subsection{Mechanistic explanations}

The mechanism of why the males and females of certain species have different spatial abilities can be described in several different ways: the ways in which their brains differ, the effects of sex hormones, possible effects of differential experience, and sex-specific cognitive styles.

\subsubsection{Neural}

\subsubsection{Sexual dimorphism in brain regions involved in spatial ability}

Due to the difficulties of experimental work on the human brain, most of the work on how sex differences in spatial ability are controlled by the brain has been carried out on laboratory rodents. The human data form a discrete set and hypothesis, which shall be discussed separately (section 1.3.1.2). 
The hippocampus is the brain region most frequently implicated in spatial learning and memory (e.g. Morris et al., 1982; Sherry et al., 1992; Poucet \& Benhamou, 1997; Pearce et al., 1998; figure 1.3). It is a discrete, evolutionarily ancient structure found in both hemispheres of the brain in birds and mammals (figure 1.4). It is thought to be involved in navigation and the representation of spatial information in an environment-centred (allocentric) framework (reviewed in Burgess et al., 1999). The rodent hippocampus has been shown to contain 'place cells'; these are neurons that only fire when the subject is in a spatially limited, stable region of its environment. The discovery of place cells led to the suggestion that the rodent hippocampus might contain a cognitive map of the environment, with different neurons relating to different spatial locations (O'Keefe \& Dostrovsky, 1971). Primates' hippocampi, however, contain 'spatial view cells' that respond to a region of visual space being looked at, independent of where the subject is located. It has been suggested that this difference between the properties of rodent and primate hippocampal cells could be due to a common mechanism operating on different visual receptive fields as rodents tend to have much larger visual fields than primates (de Araujo et al., 2001).

The associative (also called posterior) parietal cortex is also concerned with spatial cognition, although it may have a slightly different function to the hippocampus (Kolb et al., 1994; Poucet \& Benhamou, 1997; Kesner \& Long, 1998; Ellard, 2000; Chiba et al., 2002; figure 1.4). Save and Poucet (2000) gave rats with hippocampus or associative parietal cortex lesions two water maze tasks, one of which could only be solved using landmarks within the pool, the other of which had to be solved using cues from the room outside the maze. The subjects with hippocampus lesions were impaired on both tasks, while those with parietal cortex lesions were only impaired on the proximal landmark condition. This result, in conjunction with other studies, suggests that the associative parietal cortex is more involved in body-centred (egocentric) spatial cognition than the hippocampus (McDaniel et al., 1998, Save et al., 1998; Burgess et al., 1999).

Several other brain regions have also been implicated in spatial ability in rodents, including the retrosplenial cortex, perirhinal cortex, anteroventral thalamic nucleus 
and prefrontal cortex (Kolb et al., 1994; Kessels et al., 2000; Cooper \& Mizumori, 2001; Muir \& Bilkey, 2001; Harker \& Whishaw, 2002; Machin et al., 2002; Mitchell et al., 2002). Most of the evidence comes from lesion studies on laboratory rats. However, some of these regions may be involved in general encoding and retrieval, rather than specifically spatial processing (Kessels et al., 2000).

Figure 1.3: Cross-section of a rat hippocampus (after Amaral \& Witter, 1989).

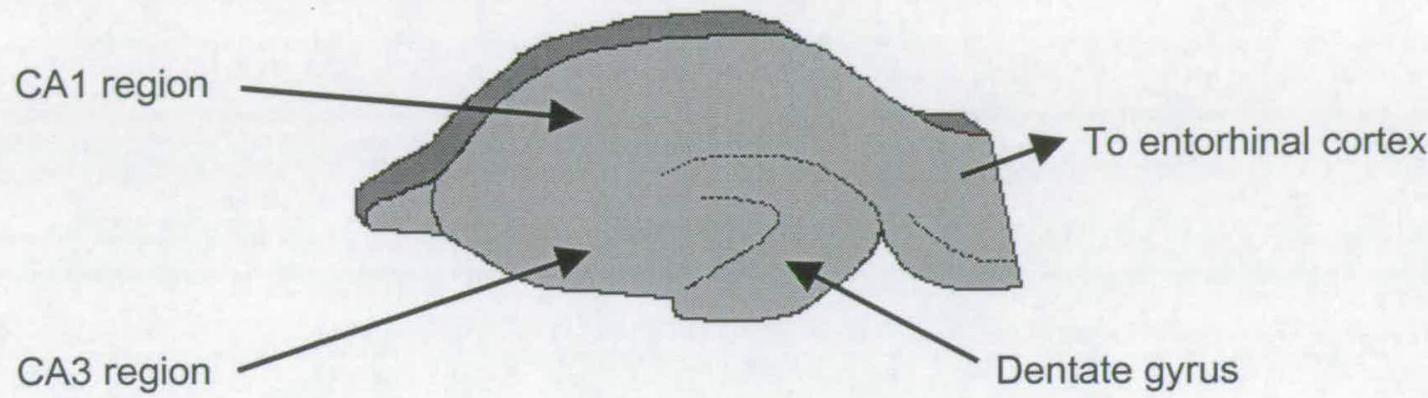

Figure 1.4: Positions of the regions in the human brain thought to be involved in spatial cognition (after Nolte, 1999).

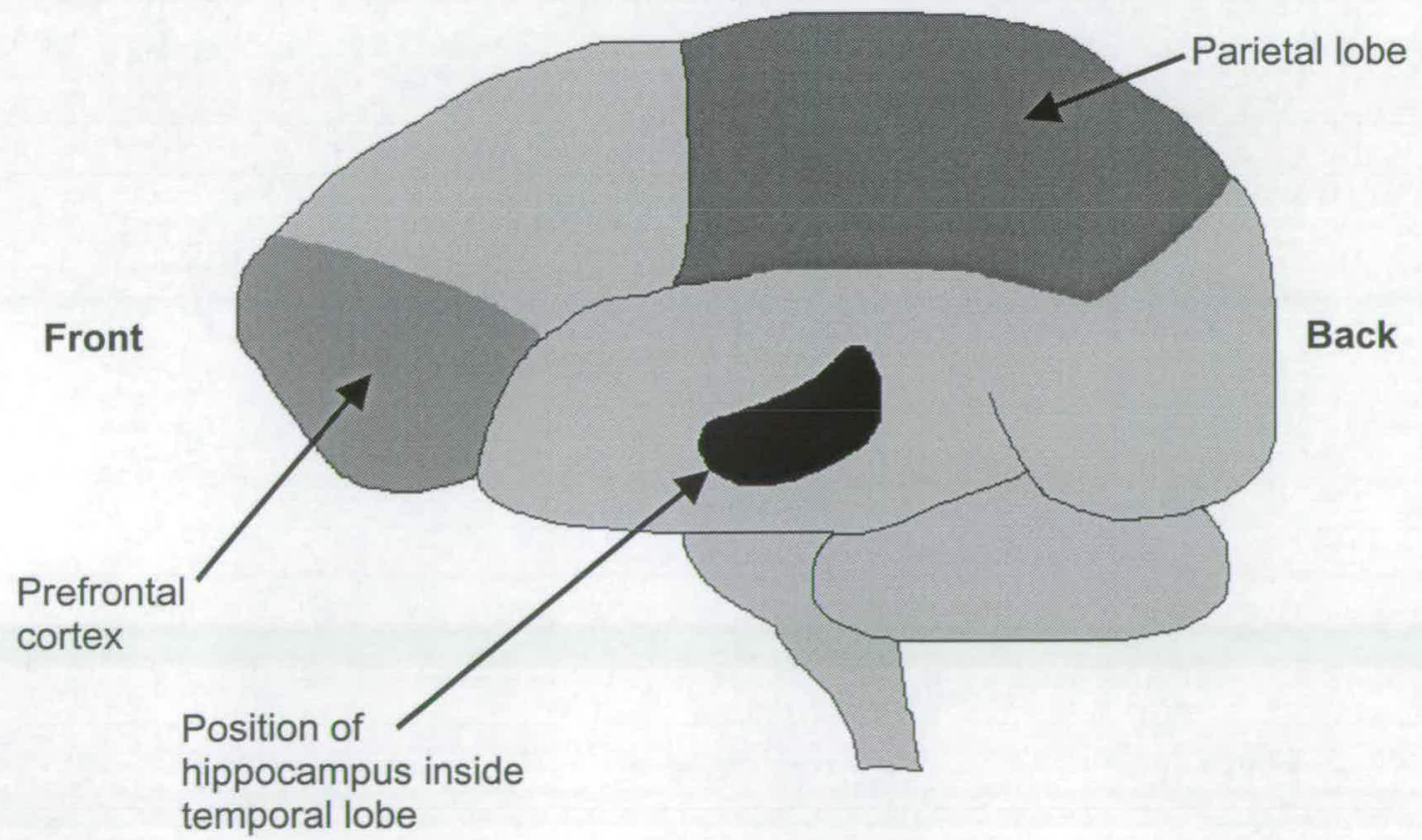


Therefore, there is good evidence that the hippocampus and various cortical areas are involved in controlling spatial ability in rodents. There is also evidence that these regions are sexually dimorphic, thus providing a possible mechanism for the observed sex differences in spatial ability.

Sexual dimorphisms have been found in the rat hippocampus in its size, in the neuronal morphology, and at the biochemical level (Loy, 1986; Diamond, 1987; Juraska, 1991; Maderia et al., 1991; Roof \& Havens, 1992). Hippocampal morphology is also sexually dimorphic in some strains of mice (Tabibnia et al., 1999). The cellular (dendritic) morphology of rat and meadow vole prefrontal and parietal cortical areas is also sexually dimorphic (Kolb \& Stewart, 1991; Kavaliers et al., 1998). The effects of frontal lesions depend on sex in rats: males given medial frontal lesions were much less impaired on MWM and RAM tasks than females (Kolb \& Cioe, 1996).

Spatial ability is also known to be correlated with hippocampal parameters: Jacobs et al. (1990) found that male meadow voles had larger relative hippocampal volumes than females, but that there were no sex differences in hippocampus size in pine voles. Male meadow voles perform better than conspecific females on spatial tasks, while there are no sex differences in spatial ability in pine voles (Gaulin \& Fitzgerald, 1986). More directly, Roof and Havens (1992) found a positive correlation between the size of the dentate gyrus granule cell layer of the rat hippocampus and performance in a MWM. Females tended to have smaller layers and poorer spatial ability than males. There is, therefore, evidence for a correlation between sexual dimorphisms in the spatial brain regions and sex differences in spatial behaviour.

\subsubsection{Functional lateralisation of the brain}

Probably due to the difficulties inherent in experimental work on the human brain, there is less evidence to provide a neural mechanism for sex differences in spatial ability in humans than in rodents. In humans, a hypothesis has been proposed that centres on hemispheric lateralisation of function. Hemispheric lateralisation refers to 
a functional asymmetry of the brain, so that an ability is more localised in one hemisphere than the other. For spatial ability, it has been suggested that the observed sex difference is due to men being more lateralised for this ability than women, and that increased lateralisation results in better spatial ability. Men have spatial ability localised in the right hemisphere, while in women it is more equally localised on both sides (Levy, 1969). This lateralisation may be due to intrinsic differences in the hemispheres, or because women tend to have a larger connection between the hemispheres (corpus callosum). The evidence for and against this theory has been discussed and reviewed many times and as it is not directly relevant to the experimental work in this thesis I shall not describe it again here (e.g. Nyborg, 1983; Iaccino, 1993; Gaulin \& Hoffman, 1998; Kimura, 1999).

There is little evidence for functional lateralisation in the rodent brain. However, in several species the areas of the brain known to be involved in spatial ability are more laterally asymmetrical in males than females. In rats, meadow voles, and some strains of mice, males have larger right dentate gyrus granule cell layers than left, while females display no such laterality (Roof \& Havens, 1992; Roof, 1993a; Galea et al., 1999; Tabibnia et al., 1999). Also in accordance with lateralisation theory, female rats have more axons in the corpus callosum than males (Juraska, 1991). If the size of a brain structure is directly proportional to the amount of processing that it does, then the lateralisation hypothesis could be an explanation of sex differences in spatial ability in rodents.

\subsubsection{Sex hormones}

Vertebrates are exposed to sex hormones in two ways. Organisational effects occur during early development (before or soon after birth) and result in "permanent designation of sex or of a sexual characteristic" (Norris, 1997). Activational hormones refer to those circulating in the subject at the time of testing; these tend to have smaller, transient effects, which fluctuate with the hormone concentration.

Testosterone is the basic androgen (male hormone) of most vertebrates, and is primarily secreted by the testis. Oestrogens and progesterone are among the most 
important female hormones; of the oestrogens oestradiol is the most biologically active and is secreted by the ovaries. Confusingly, testosterone can be locally metabolised to oestradiol, and therefore can activate either androgen or oestrogen signalling pathways in the brain. Adult males have high levels of circulating testosterone and low levels of circulating oestradiol, most of which have arisen from conversion of testosterone. Adult females also have measurable levels of circulating testosterone (in humans, approximately ten times lower than those seen in normal males) as well as circulating oestrogens and progesterone, the levels of which vary with fertility cycle phase.

\subsubsection{Organisational hormones}

\section{Testosterone}

Manipulations of the gonadal hormone levels to which neonatal rats are exposed affect spatial ability. The administration of testosterone or oestradiol to newborn female rats increases adult female spatial ability (Dawson et al., 1975; Stewart et al., 1975; Joseph et al., 1978; Williams et al., 1990; Roof, 1993b). Neonatal administration of testosterone to males decreases adult spatial ability (Roof, 1993b). Similar effects are seen when exposure to gonadal hormones before birth is manipulated (Isgor \& Sengelaub, 1998). Male neonatal castration, which removes the major source of testosterone, decreases spatial ability in rats (Dawson et al., 1975; Joseph et al., 1978; Williams et al., 1990).

Before birth foetuses are naturally exposed to testosterone from up to three sources from the mother, from their own developing gonads (if they are male), and in some species from male littermates. Therefore, individuals from litters containing more males than females will be exposed to more prenatal testosterone than individuals from litters containing mainly females, and this could affect spatial ability. Female rats from litters with a high proportion of male foetuses perform more accurately during the acquisition of a RAM task than females from litters with a low proportion of male foetuses (reported in Williams \& Meck, 1991). Similarly, meadow voles of 
both sexes from a male-biased litter performed significantly better as adults on a MWM task than voles born into a female-biased litter (Galea et al., 1994b; 1996).

Together these results suggest that there is an optimal level of organisational testosterone for maximum adult spatial ability, which is approximately the level experienced by normal males (or perhaps normal males from a male-biased litter). The fact that oestradiol administration has the same effect as testosterone suggests that it is not testosterone itself, but its metabolite, that is having the effect.

Human studies have centred on observations of people who have conditions that result in them being exposed to abnormal levels of hormones during early development. The conditions studied include androgen insensitivity syndrome (equivalent to having no androgens) and congenital adrenal hyperplasia (overproduction of androgens). The results from these studies are consistent with the rodent work: increased organisational testosterone results in higher spatial ability in females and lower spatial ability in males, while reductions in organisational testosterone are correlated with lower than average spatial ability in males (Hampson et al, 1998; Kimura, 1999).

\section{Oestrogens and progesterone}

Studies that examine the possible organisational influences of hormones secreted by the ovaries are rare. This is probably because the default developmental sex is female in mammals. The female brain has therefore been considered the default option, i.e. what develops in the absence of testicular hormones. However, recent work suggests that ovarian hormones can have significant effects on the development of sexually dimorphic brain structures (Fitch \& Denenberg, 1998) and so they may be important in the organisational development of female-type spatial ability.

Turner syndrome is a condition in humans where the patients are female but have only a single $\mathrm{X}$ sex chromosome and have impaired ovarian function from early in life. Turner women tend to be worse than normal women at spatial tasks, even if they are receiving (activational) hormone replacement therapy (Collaer et al., 2002). 
This suggests that there may be an organisational role for ovarian hormones in female sexual differentiation.

Hull et al. (1980) gave female rats progesterone implants during pregnancy and lactation, and found that the male offspring had impaired spatial performance on a maze task when tested as adults, compared to the offspring of dams given placebo implants. The spatial ability of the female offspring was unaffected by progesterone treatment.

\subsubsection{Activational hormones}

Activational hormone levels are the concentrations of hormones experienced by the subject at the time of testing. These do not seem to have as substantial an effect on spatial ability as organisational hormone levels. For example, sex differences in spatial abilities have been found in rats that were gonadectomised as adults even though this means that the major activational source of sex hormones was removed (Williams et al., 1990; Luine \& Rodriguez, 1994). Sex differences can also be found in prepubertal rats, which have low levels of activational sex hormones (Roof, 1993a; Kanit et al., 1998b).

\section{Testosterone}

In spite of the evidence linking testosterone levels to spatial ability there are few studies looking at the influence of activational testosterone levels on spatial ability in rodents, even though testosterone levels in rodents have been shown to vary from month to month (Moeller et al., 1988). Gonadectomised adult male rats given testosterone implants performed better on a T-maze than gonadectomised males given placebo implants (Kritzer et al., 2001). Similarly, Galea et al. (1994a) found that male deer mice in breeding condition (associated with high testosterone levels) performed significantly better in the MWM than males in non-breeding condition. Conversely, Galea et al. (1995) found that there were no significant performance differences between male meadow voles with high and low levels of plasma testosterone. This may have been because the meadow voles were assigned to high and low testosterone groups based on a median split of plasma testosterone levels. 
The magnitude of difference in plasma testosterone levels may be much greater between breeding and non-breeding males than between the high and low testosterone groups used in this study. Also, testosterone was not assayed until two to four weeks after behavioural testing and so may not represent the true levels at the time of testing. Taken together these studies suggest that spatial ability is positively correlated with testosterone levels.

There are many studies of activational testosterone effects in humans. Some have looked for correlations between testosterone levels and spatial ability while trying to control for the variables that may influence testosterone or cognitive performance (e.g. Neave et al., 1999). Others have looked at groups of people given exogenous hormones (e.g. transsexuals, Van Goozen et al., 1995; women given testosterone, Postma et al., 2000). Still others have measured testosterone and spatial ability at two separate times and compared the changes within individuals (across seasons, Kimura \& Hampson, 1994; across a day, Moffat \& Hampson, 1996; Silverman et al., 1999; Hausmann et al., 2000; Cherrier et al., 2001). The consensus from these studies is that there is a curvilinear relationship between activational levels of testosterone and spatial ability, with maximal spatial ability found in the low male range.

\section{Oestrogens and progesterone}

There has been a lot of research on activational hormone levels in female humans and rats due to the regular natural fluctuations in concentration that they experience during their fertility (oestrous or menstrual) cycles (figure 1.5). The consensus from studies of fertility cycles in humans, rats and rhesus monkeys is that spatial skills are reduced at the high oestrogen and progesterone phases (pro-oestrus or luteal) relative to the rest of the cycle (Hampson \& Kimura, 1988; Frye, 1994; Moody, 1997; Hausmann et al., 2000; Lacreuse et al. 2001; McCormick \& Teillon, 2001; Phillips \& Silverman, 1997; Warren \& Juraska, 1997). However, others have found the opposite effect: that spatial ability was enhanced during high oestrogen cycle phases (Healy et al., 1999; Postma et al., 1999; Frick \& Berger-Sweeney, 2001). Frye (1994) gave hormone implants that mimicked hormone levels of the oestrus and di- 
oestrus phases of the rat oestrous cycle to ovariectomised female rats. The 'oestrus' subjects were impaired on a water maze task compared to the 'di-oestrus' subjects.

Figure 1.5: Changes in progesterone and oestradiol (an oestrogen) across the rat oestrous cycle (after Norris, 1997).

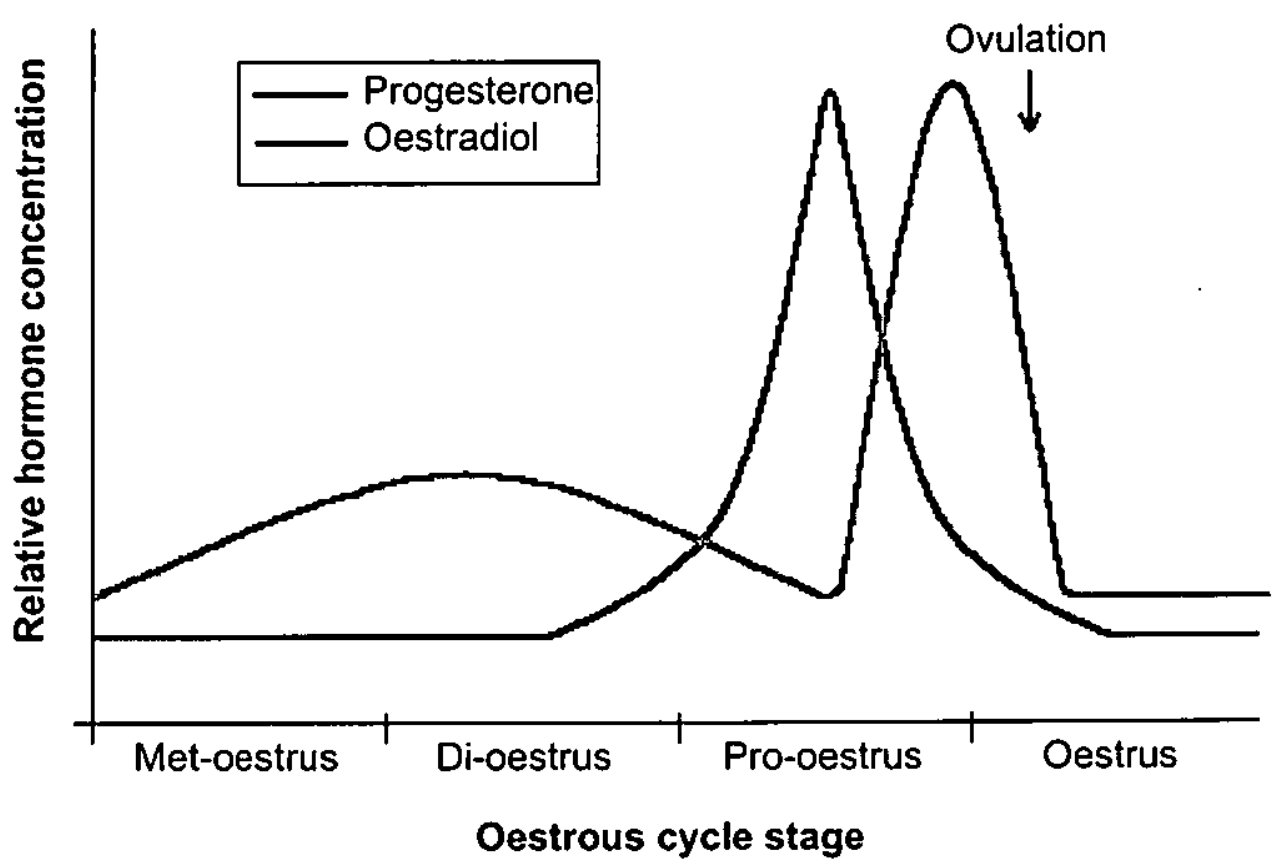

There are also a number of studies that have found no relationship between fertility cycle and spatial ability. This latter group includes studies that assayed hormone levels to determine cycle stage so the lack of a relationship between spatial ability and stage of fertility cycle cannot, therefore, be attributed solely to inaccurate methods of cycle phase determination (Gordon \& Lee, 1993; Berry et al., 1997; Stackman et al., 1997; Epting \& Overman, 1998; Halpern \& Tan, 2001; Mumenthaler et al., 2001). It is not clear whether the inconsistency in observing the spatial ability fluctuations is due to variation in experimental testing conditions, or because the effect is small and easy to miss.

Unlike rats, deer mice and Microtus spp. have a seasonal period of fertility, rather than a regular oestrous cycle. Galea and colleagues tested breeding and non- 
breeding deer mice in a MWM and only found sex differences in latency to complete the maze during the breeding season (Galea et al., 1994a; Galea et al., 1996). This is when the range size varies between the sexes and, presumably, levels of gonadal hormones are higher. Female performance was significantly better in the nonbreeding subjects compared to deer mice in breeding condition. This finding has been replicated in meadow voles: female meadow voles with high oestradiol levels exhibit longer latencies to find the hidden platform in a MWM than females with low oestradiol levels (Galea et al., 1995; 1996). High oestradiol levels are associated with females in breeding condition.

Peaks in oestrogen and progesterone occur at similar times during natural fertility cycles (figure 1.5) so it is difficult to separate the effects of these two hormones. In an attempt to do this, ovariectomised female rats were given hormone injections and then tested on a water maze task (Chesler \& Juraska, 2000). Neither oestrogen nor progesterone alone impaired spatial performance relative to control animals that were given a placebo injection, only subjects given a combination of oestrogen and progesterone had impaired spatial ability. Conversely, Sandstrom and Williams (2001) found that when ovariectomised female rats were given injections of oestradiol and progesterone their memory for a water maze task was enhanced. Hausmann et al. (2000) compared concentrations of circulating oestradiol and progesterone with spatial ability in women at different menstrual cycle phases. They found a negative relationship between spatial ability and oestradiol levels, but no relationship between progesterone and spatial ability.

The most consistent correlation is a negative one between oestrogen and spatial ability, although progesterone may also have an effect. Oestrogen and/or progesterone only seem to have a detrimental effect on spatial ability when they are high, short-term changes. Studies in which oestrogens are given in low doses over a long time (chronic oestrogen replacement) tend to find an improvement in spatial ability. This effect is seen in women (for example hormone replacement therapy), female rats, and female rhesus monkeys (Daniel et al., 1997; Bimonte \& Denenberg, 1999; Duff \& Hampson, 2000; Gibbs, 2000; Lacreuse et al., 2002). 
Just as females generally have low levels of circulating testosterone, males generally have some oestrogens in their blood because oestrogens are produced by the testes and adrenal glands as well as by the ovaries. There is evidence that activational oestrogen levels in males may influence spatial ability. Silverman et al. (1996) report a study that found an inverse relationship between oestrogen levels and performance on a mental rotation task in men. Similarly, Kritzer et al. (2001) found that gonadectomised male rats given oestradiol implants took longer to learn a Tmaze than either intact controls, or gonadectomised subjects treated with testosterone.

\subsubsection{Hormonal effects on the brain}

There is evidence that brain regions controlling spatial ability differ between the sexes, and also that sex hormones affect spatial ability. In this section I summarise the evidence that sex hormones affect regions of the brain involved in spatial ability, thus providing a mechanism whereby sex differences in spatial ability could develop.

\section{Organisational hormones and the brain}

Organisational levels of sex hormones have been shown to influence the development of the hippocampus. Roof and Havens (1992) found that neonatal testosterone treatment made the female hippocampus look more like a typical male's, by increasing the size of the dentate gyrus granule cell layer and making it laterally asymmetrical. Sex differences in CA1 and CA3 (figure 1.3) pyramidal cell field volumes and soma sizes can be reversed by prenatal administration of testosterone to females and flutamide (an anti-androgen) to males (Isgor \& Sengelaub, 1998).

The prefrontal cortex is also affected by organisational hormones: male and female rats gonadectomised immediately after birth have changes in the dendritic branching of cells in their prefrontal cortices compared to control males and females (Kolb \& Stewart, 1991).

\section{Activational hormones and the brain}


Activational hormone levels also affect the brain regions involved in spatial ability. Natural seasonal fluctuations in testosterone and oestradiol are correlated with cell proliferation in the dentate gyrus of the hippocampus in meadow voles (Galea \& McEwen, 1999). Testosterone and oestradiol levels are also correlated with the width of the dentate gyrus as well as the overall volume of the hippocampus (Galea et al., 1999). Males with high testosterone levels and females with high oestradiol levels tend to have larger hippocampal volumes, and high testosterone levels in males are associated with having a wider dentate gyrus.

The fluctuations of hormones across the oestrous cycle affect hippocampal neurons. The density of dendritic spines on hippocampal CA1 pyramidal cells fluctuates over the rat oestrous cycle: the lowest density is in the oestrus phase when oestradiol and progesterone levels are at their lowest (Woolley et al., 1990a). Synaptic plasticity (measured in terms of long-term potentiation and long-term depression) also varies across the oestrous cycle in the CA1 region of the hippocampus (Warren et al., 1995; Good et al., 1999).

Activational hormone levels also affect non-hippocampal brain regions involved in spatial ability. Female rhesus monkeys that are ovariectomised as adults have fewer axons in the prefrontal cortex than intact females. If ovariectomised subjects are given oestrogen and progesterone hormone replacement the density is restored, oestrogen treatment alone results in only partial restoration (Kritzer \& Kohama, 1998).

\subsubsection{Environment}

So far I have examined sex differences in spatial ability as if they are innate, i.e. they develop irrespective of the environment encountered by the organism. However, it is also possible that spatial abilities vary between the sexes because individuals of each sex have a different experience of the environment and develop different levels of spatial ability during their lifetime (Sherman, 1967).

Spatial experience can affect spatial ability. In humans, scores on a specific spatial test can be improved with practice (e.g. Conner et al., 1978; Baenninger \& 
Newcombe, 1989). Studies on humans have also compared the amount of spatial activities that an individual participates in and their scores on spatial tests, and have found a significant positive correlation between spatial activities and performance on spatial tests (Olson et al., 1988; Baenninger \& Newcombe, 1989). Enriching the home cages of laboratory rats by providing larger cages, companions, and/or toys such as tunnels and ladders results in superior spatial ability when individuals are compared to subjects raised in standard laboratory cages (Juraska et al., 1984; Seymoure et al., 1996; Nilsson et al., 1999). This effect could be due to the greater spatial experience of the subjects kept in enriched cages, but may also be explained by other factors such as the stress caused by being kept in deprived conditions. Exercise can also improve spatial ability (Anderson et al., 2000). However, Gaulin and Wartell (1990) found no differences in maze performance between wild caught prairie voles and their first generation laboratory reared offspring, even though the wild voles were likely to have had more spatial experience.

As spatial experience does appear to improve spatial ability, it could result in sex differences in spatial ability if the sexes have different spatial experiences or are differently affected by the same experiences. Conner et al. (1978) found that girls benefited more from practice on a spatial task than boys, and that practice eliminated sex differences in performance, but apart from this finding there is little evidence that experience causes sex differences in spatial ability. However, there is evidence to suggest that most of the sex differences seen in spatial ability are due to developmental processes rather than sex-specific experiences. First, sex differences in spatial tests in humans tend to be consistent in magnitude and direction across the world despite cultural differences in childhood experiences (Silverman et al., 1996). Second, the fact that sex differences can be found in laboratory animals that have been kept in controlled conditions where males and females cannot have had any different experiences, is also strong evidence that sex differences in spatial ability are not due to difference in experience between the sexes (e.g. Luine \& Rodriguez, 1994; Cimadevilla et al., 1999). 
It is possible that environmental effects other than spatial experience could cause sex differences in spatial ability. Endo et al. (1994) claim that sex differences in spatial ability in laboratory rats are due to them being fed a hard pellet diet, as the sex differences disappear when the rats are feed powdered food. They explain this as being due to chronic stress in female rats caused by hard food, as females fed hard food had enlarged adrenal glands. This hypothesis has not been addressed by any other study, and it is difficult to explain why only females should be stressed by being fed hard food and why this stress should result in reduced spatial ability.

To conclude, spatial ability can be affected by spatial experience. However, sex differences in spatial ability can be found in rats even when both sexes have had identical experiences. This suggests that although experience may be able to modify sex differences in spatial ability, the observed sex difference is greatly determined by biological factors, at least in rodents.

\subsubsection{Cognition}

Not only are there morphological and physiological differences in brain regions between the sexes, it has been suggested there may also be differences in the way the sexes use their brains to solve spatial problems. Studies with rats and with humans have shown that the sexes tend to learn different aspects of their spatial environment.

Roof and Stein (1999) used a working memory task in the MWM where each rat underwent two trials with a one-hour inter-trial interval, per day. The platform position was constant for both of the trials on a single day, but the release position was not. There were no sex differences in ability providing that the experimenter stood in the same position at the side of the maze during both the trials. If the experimenter moved between trials, the females took a longer path to the platform than the males during the second trial. Further analysis of the data showed that this was because the female rats spent longer in the correct quadrant relative to the experimenter, rather than the correct quadrant relative to the other room cues.

The findings of Roof and Stein's (1999) study are consistent with earlier work on rats: Williams et al. (1990) found that female rats used both landmark (movable 
objects in the room) and geometrical (the shape of the room) cues to solve a RAM, while male rats used only geometrical room cues. They proposed that female rats might take longer to learn the maze because they learn more cues. Kanit et al. (1998a) suggested that females use local visual cues to solve a MWM test, while males use navigational (distant) cues. Similar results have also been found in later studies on rats, and in humans and kangaroo rats (Galea \& Kimura, 1993; Langley, 1994; Lawton, 1994; Dabbs et al., 1998; Montello et al., 1999; Kanit et al., 2000; Tropp \& Marcus, 2001b; Saucier et al., 2002). Females rely on landmarks, while men tend to use geometric and directional information. Taken together these studies suggest differences between the sexes in the cues used to solve a spatial problem, with females relying more heavily on actual objects than males.

There also appear to be differences in the brain regions used by males and females to solve spatial tasks. Brain lesions can have different effects on spatial ability in male and female rats. Roof et al. (1993) gave rats lesions of the entorhinal cortex, which is an area with major connections (both inputs and outputs) to the hippocampus (Burgess et al, 1999, figure 1.3). They found that male rats were more impaired on the MWM than females with the same lesions. No sex differences in maze ability were found in sham-operated control rats. Lesions to the frontal cortex had more disruptive effects on performance of female rats in both the MWM and RAM than they did on the performance of male rats (Kolb \& Cioe, 1996). These results may be due to males and females having different reactions to lesions in different brain areas, but they are also consistent with the cognitive style hypothesis if the information regarding the different cues is processed in different areas of the brain. A recent study that visualized activity in the human brain during a virtual reality maze task also found sex differences in the brain regions used. Many regions were active in both sexes during the task, such as the hippocampal areas and parts of the parietal lobes, but there were also sex-specific regions of activity. Males used their left hippocampus, while females showed activity in the right parietal and right prefrontal cortex (Gron et al., 2000). 
These separate lines of evidence consistently point to two ways of solving spatial problems, which seem to be found in both humans and rodents. The 'male-typical' cognitive style may activate the hippocampus to use distant navigational or geometric cues. The 'female-typical' cognitive style, on the other hand, may use the parietal and prefrontal cortex to learn and recall more local, landmark cues.

\subsubsection{Conclusion}

Sex differences in spatial ability seem to be caused by the effects of both activational and organisational gonadal hormones on the parts of the brain involved in spatial cognition (table 1.4). Sex differences in cognitive style may be able to explain sex differences in spatial ability in terms of differences in the way that the sexes process the information involved in a spatial task. However, this hypothesis has yet to be explicitly tested. Sex specific experiences may affect spatial ability, but these seem to be unlikely to be a primary cause of observed sex differences in spatial ability.

Table 1.4: Summary of the effects of organisational and activational sex hormones on spatial ability.

\begin{tabular}{l|l|l} 
Hormone & Timing & Effect on spatial ability \\
\hline Testosterone & Organisational & Optimal level in normal male range \\
Activational & Optimal level in low normal male range \\
Oestrogen & Organisational & $\begin{array}{l}\text { Unknown, extremely low levels may impair female } \\
\text { performance }\end{array}$ \\
Progesterone & Octivational & $\begin{array}{l}\text { Negative correlation } \\
\text { Unclear, extra impairs male performance whilst not }\end{array}$ \\
& Activational & $\begin{array}{l}\text { affecting female } \\
\text { Unclear, possibly negative correlation }\end{array}$
\end{tabular}

Laboratory rats are often the subjects of choice for studies looking at the mechanism of sex differences in spatial ability. However, sex differences are not consistently 
found in studies of unmanipulated individuals (table 1.2). This is probably due to their long history of domestication. It is possible that sex differences in spatial ability do not exist in normal laboratory rats and that the differences that have been reported are a result of the incidental manipulation of factors that influence spatial ability. These factors could include organisational testosterone levels due to different litter sex ratios, or activational hormone levels due to different times of the year (testosterone) or stages of the female oestrous cycle (oestrogens). The observed sex differences may also be a by-product of the way spatial ability is tested. Variation in these factors may be further confounded by the use of different strains, which may in turn be differentially susceptible to the factors.

\subsection{Alternative explanations}

The evolutionary hypotheses all assume that sex differences in spatial ability are adaptive products of natural selection. However, it is also possible that the observed sex differences in spatial ability are a by-product of selection for another feature (Gould \& Lewontin, 1979). The way that spatial ability is tested, especially in rodents, means that spatial ability could be confounded with another feature that shows variation between the sexes, i.e. the tests might not be testing differences in spatial ability, but rather differences in another trait.

One possibility that has received some attention is the role that stress may have in the production of sex differences on spatial tasks (Perrot-Sinal et al., 1996). As males and females often respond differently to stress, and acute stress is known to impair learning of spatial tasks, it is possible that sex differences in spatial abilities are due to differences in stress responses (reviewed in Shors, 1998). There seems to be some support for this theory.

First, sex differences in rats are often only apparent during the initial stages of the task. Females tend to take longer than the males to reach an asymptotic degree of accuracy, but, once reached, there is no significant difference between the sexes' performance. For example, Williams et al. (1990) compared the performance of normal male and female rats in a RAM with 8 out of 12 arms rewarded. They found 
differences between the sexes in the number of choices required in order to obtain all the food only during the first three blocks of three trials. On the following three blocks of trials the males and females were performing equally well and seemed to have reached asymptotic performance. Males seemed to reach this asymptote a block earlier than females. Interestingly, this effect does not tend to be apparent in studies using meadow voles or deer mice (e.g. Galea et al., 1994a; 1994b; 1995). In these studies, sex differences in performance are seen consistently across the blocks. In rats, acquisition-only sex differences are seen in both RAM and MWM studies (Juraska et al., 1984; Williams et al., 1990; Williams \& Meck, 1991; Seymoure et al., 1996; Kanit et al., 1998b; Markowska, 1999). There are several possible explanations for only finding sex differences during the acquisition phase of a test. It could be due to females taking longer to learn because they are more stressed by the novelty of the maze. However, it could also be due to there being sex differences in learning but not in memory, or that the task is not difficult enough to reveal sex differences once it is learnt.

Second, pretraining may influence sex differences in the MWM. A study by PerrotSinal et al. (1996) found sex differences in path length to reach the platform in a MWM using rats that did not receive pretraining. However, these differences were not found in a group of rats that received training with a non-stationary hidden platform in the water maze prior to the spatial learning trials. The rats that did not receive pretraining took five blocks of four trials to learn the platform's location, while those that had pretraining learned in two blocks. None of the MWM studies that used extensive pretraining found sex differences (Bucci et al., 1995; Warren \& Juraska, 1997; Healy et al., 1999; Markowska, 1999). However, this cannot be the whole answer as Kanit et al. (1998a) did not use pretraining and still failed to find sex differences.

The third and final line of evidence that stress may cause apparent sex differences in spatial ability in rodents comes from experiments involving opioid manipulation. Increased endogenous opiate activity is associated with stress. In both meadow voles and deer mice administration of an opiate antagonist, or exposure to extremely low 
frequency magnetic fields (which also have an inhibitory effect on opiate function) improves female performance in the MWM and reduces sex differences (Galea et al., 1994c; Kavaliers et al., 1996). One interpretation of these data is that female rodents are more affected by spatial ability impairing opiates caused by stress, than males.

Taken together these studies suggest that female rodents are more stressed than males by maze tasks and this impairs their apparent spatial ability. This would be especially true in the early stages of testing when the task is novel. However, in tasks designed to measure stress levels, such as open-field tests and the elevated plus maze, male rats often score higher for stress than females (e.g. Barrett \& Ray, 1970; Stewart et al., 1975; Johnston \& File, 1991). Although these findings are in conflict with the above studies, they can be explained. First, these tasks were developed to measure stress in male rats, and factor analyses have shown that they may measure different variables, and therefore not be a valid test of stress, in females. Female rats' scores on such tasks are more related to activity than stress (Fernandes et al., 1999). Second, the same level of stress may have different effects on the cognitive abilities of males and females. Male rats may be able to be subjected to high levels of stress before their spatial ability is impaired, while it is possible that females may show reduced in spatial ability at much lower stress levels.

A sex difference in activity is another possible cause of the observed sex differences in spatial ability. Female rats have consistently been shown to be more active than male rats on wheels and in open-fields (Barrett \& Ray, 1970; Dawson et al., 1973; Magalhaes \& Carlini, 1974; Dawson et al., 1975; Stewart et al., 1975; Krasnoff \& Weston, 1976). Many of the mazes used to test spatial ability may confound results with differences in activity between subjects. This is especially true when time to complete the maze (latency), is used as the sole measure of ability.

There is, however, evidence against sex differences in activity affecting spatial ability. First, Kanit et al. (2000) found no sex differences in rats in a swim speed test (although they also did not find sex differences in spatial ability). Second, the activity and error production of prairie and meadow voles in a Davenport maze are not correlated (Gaulin et al., 1990). Lastly, possible sex differences in activity can 
only really explain the observed sex differences in rodent mazes, not in humans where the tests do not have a major activity component.

Wynn et al. (1996) suggested that sex differences in spatial cognition are an evolutionary by-product of selection for optimal rates of foetal development. Their theory is that sex differences in spatial ability are a result of sex differences in brain asymmetry and laterality of function, which are in turn a result of developmental timing related to growth of the hemispheres and ambient levels of testosterone. If selection acted to change the timing of foetal development, this could change the timing of exposure to testosterone and so affect hemispheric growth and spatial ability. Wynn et al. (1996) do not have a strong hypothesis as to why the rate of foetal development should have undergone selection or why this selection should differentially affect males and females.

Although there seems to be little convincing evidence for activity differences underpinning the production of sex differences in spatial cognition, the results to date, from rats at least, could be explained by stress as the causal factor.

\subsection{Conclusions}

Sex differences in spatial ability are an exciting and fast moving multi-disciplinary area of research. Many theories (both evolutionary and mechanistic) have been proposed to try to explain sex differences in spatial ability, but experimental results are often few and contradictory. In this thesis I will address what I consider to be some of the most interesting questions raised by this review, namely: why are sex differences in spatial ability not consistently found in rats, and can alternatives such as stress provide an explanation? Does the range size hypothesis' prediction of better male spatial ability in Mongolian gerbils (Meriones unguiculatus) hold true? Does the female foraging hypothesis really refer to spatial ability, or can women's better performance on object-location tasks be explained by their superior memory for objects? 


\section{Chapter 2: Are there differences in the ways that male and female laboratory rats learn spatial tasks?}

\subsection{Introduction}

Although laboratory rats are often used in studies of sex differences in spatial ability, sex differences are not consistently found in un-manipulated (control) rats even within the same laboratory (e.g. Roof, 1993b; Luine \& Rodriguez, 1994; Kolb \& Cioe, 1996; Van Haaren et al., 1987; Kanit et al, 1998a, 1998b). The evolutionary hypotheses discussed in chapter 1 do not provide any strong predictions about sex differences in spatial ability in laboratory rats. There is some evidence that male rats may have larger home ranges, which would result in a prediction of superior male spatial ability from the 'range size' hypothesis, but these data have not been confirmed (Taylor, 1978; Macdonald \& Fenn, 1995). There is also the problem of rats' long history of domestication - most of the strains used today were developed in the early twentieth century (Koolhaas, 1999). Artificial selection may have altered characteristics created by natural selection in the laboratory rats' wild ancestors in unpredictable ways.

Therefore, it seems possible that there are no sex differences in spatial ability in laboratory rats. The inconsistent study results might be due to differences in experimental methods between laboratories, such as having more or fewer landmarks, using different strains, using rats that are used to being handled to a greater or lesser extent, or differences in food restriction regime (Williams et al., 1990; Andrews, 1996; Miller \& Dess, 1996; Perrot-Sinal et al., 1996). Van Haaren et al. (1987) suggested this possibility more than 15 years ago, but little research has been done to test it. I decided to test whether some of these possibilities could result in sex differences in spatial ability in laboratory rats.

When they are found, sex differences in spatial ability in laboratory rats are often only present during the initial stages of a maze task. Females tend to take longer 
than males to reach an asymptotic performance, but, once reached, there is no significant difference between the sexes. This is apparent in both RAM and Morris water maze (MWM) studies (section 1.4; e.g. Juraska et al., 1984; Williams et al., 1990; Williams \& Meck, 1991; Seymoure et al., 1996; Kanit et al., 1998b; Markowska, 1999).

There are several possible explanations as to why sex differences are only apparent during the acquisition phase of a test. First, it may be that the tasks are too easy and have a ceiling level of performance below both sexes' maximum performance. However this seems unlikely, as acquisition-only sex differences have been found on a mixture of tasks of varying difficulty. Second, there may be sex differences in the rate of learning a spatial task, but not in memory once the task is learnt. There are several aspects that the subject has to learn during the early stages of maze training including the maze procedure (i.e. what is involved and how to solve the task), the landmarks in the room, and the position of the rewards within the maze. It is possible that females may take longer to learn some or all of these features (Williams et al., 1990; Kanit et al., 1998a). Third, there could be sex differences, not in spatial ability, but in the subject's response to the novel environment provided by the maze. This could affect apparent spatial ability in the initial stages, but not once the subject has become habituated to the task. There is some evidence that the sexes might respond differently to stress caused by the maze procedure (section 1.4).

In order to determine which, if any, part of learning to solve a spatial task results in sex differences in spatial ability I repeatedly trained rats to find rewarded locations in both a RAM (with four out of eight arms rewarded) and a MWM. Both the RAM and the MWM were used in this experiment because, although they are both tests of spatial ability, they are quite dissimilar. This difference is most notable in the type of motivation used (aversive in the MWM and appetitive in the RAM), and in their difficulty - in a typical MWM protocol the subject only has to remember a single location, while in the partially rewarded RAM several locations need to be remembered in order to solve the maze (Stewart \& Morris, 1993; Foreman \& Ermakova, 1998; D'Hooge \& De Deyn, 2001). As the two mazes probably test 
different aspects of spatial ability (Hodges, 1996; Dudchenko et al., 1997) the experiment was repeated in each maze, in order to get an overview of the possible causes of sex differences in spatial ability.

Subjects were trained on two consecutive patterns of rewarded arms in the RAM and three consecutive platform locations in the MWM. During the first training stage they were exposed to the full set of influences that could affect the sexes differently, including stress associated with the novel task, learning the procedure, learning the landmarks, and learning the location of the reward. Sex differences in performance at this stage could be due to any of these factors. During the second training stage only the position of the reward(s) was changed. The subjects were already familiar with the novel environment provided by the maze, had learnt the procedure, and had learnt the landmarks in the room. Therefore, I expected to find sex differences at this stage only if there were differences in the sexes' ability to learn the position of the reward(s) within the maze.

As training in the MWM is significantly faster than training in the RAM a third stage was introduced in the MWM study, where the room cues were disturbed as well as the location of the reward being moved. If sex differences were found at this stage, but not during the second training stage, it is likely to be due to differences in the rate that the sexes learn the extra-maze cues used to solve the maze.

To summarise, if sex differences in spatial ability were found on the first stage, but not the second or the third, I predicted that they were likely to be due to differential effects of stress or learning the procedure. If sex differences were found on all three of the stages, I predicted that they would be due to differences in the sexes' ability to learn the rewarded position(s). If sex differences were found on the first and third, but not the second, stage in the MWM, I predicted that they would be due to sex differences in the rate of learning the extra-maze cues. 


\subsection{Radial arm maze experiment}

\subsubsection{Pilot study}

A pilot study using the RAM was carried out during April-June 2000. Subjects were four (two male and two female) experimentally naïve out-bred Wistar (Hanover Origin) rats from B\&K Universal Ltd., weighing 175-200g upon arrival in the animal house and housed individually. They were food deprived to $90 \%$ of their freefeeding weight, given pretraining in the RAM and trained on two consecutive patterns of four rewarded arms. This allowed me to gain experience with both animal handling and the RAM protocol.

\subsubsection{Materials and methods}

\section{Subjects}

The main RAM experiment was carried out during September-December 2000. Ten male and ten female experimentally naïve Sprague-Dawley rats were supplied by B\&K Universal Ltd.. This albino strain was chosen because it is commonly used in studies of sex differences in spatial ability (e.g. Williams et al., 1990; Roof et al., 1993; Isgor \& Sengelaub, 1998). Although aged albino rats tend to lose their visual acuity, which may interfere with their ability to learn spatial tasks, young albino rats are able to learn spatial tasks just as well as melanic strains such as the Lister Hooded (reviewed in Andrews, 1996; D'Hooge \& De Deyn, 2001). The rats were bought by weight rather than by age, both sexes were $175-200 \mathrm{~g}$ when delivered. Buying by weight rather than age meant that the females, which tend to put on weight more slowly than males, might have been slightly older than the males. However, literature provided by the supplier suggests that both sexes are young adults at this weight and this is the age when sex differences are likely to be greatest.

The subjects were housed in the animal house in same-sex pairs in standard rack cages with plastic bases and wire lids ( $410 \mathrm{~mm}$ long, $250 \mathrm{~mm}$ wide, $200 \mathrm{~mm}$ high), a layer of wood-chips covered the floor. They were given clean cages every week and tap water was available ad libitum throughout the experiment. Harlan 41B pelleted 
food was available ad libitum except during food restriction. The animal house was lit by fluorescent tubes on a 12:12 light:dark cycle (lights on at 0530) and was kept at a temperature of $21-22^{\circ} \mathrm{C}$. Subjects were marked with coloured ink on their tails to enable identification.

The subjects were allowed to settle into the animal house for four days before being weighed and put on the food restriction regime. The target weight ranges for each week were calculated by adding 10-15g per week for the males, and 5-10g per week for the females, to the previous week's weight (or $90 \%$ of the current weight in the case of the first week). These weight gains were calculated from age/weight graphs supplied by B\&K Universal Ltd. The rats were weighed every day between 0900 and 1030 until their weight had stabilised (two weeks), when pretraining was started. During the experiment rats were weighed three times a week before the day's trials, and their food intake adjusted so they met their target weight for the week. This also allowed the pairs to be monitored in order to check that both partners had a similar weight gain. It was found that all the rats tended to gain weight albeit at slightly different rates. As different rates of weight gain would be expected under freefeeding conditions I assumed that no subject was having its food intake severely restricted by its cage-mate. Immediately prior to pretraining the rats were introduced to the food cups used in the maze by leaving one in each cage overnight, and to the food rewards by feeding each subject two rewards after weighing for five days before pretraining began.

\section{Maze}

The RAM used in this experiment was locally made. It consisted of eight arms measuring $800 \mathrm{~mm}$ long by $80 \mathrm{~mm}$ wide with $200 \mathrm{~mm}$ high sides, which could be slotted into an octagon-shaped central compartment (figure 2.1). The floor of each arm was opaque green plastic, while the sides of the arms were made from clear plastic and so prevented escape whilst allowing the subject a clear view of the room. The octagonal central compartment was made from opaque green plastic, it was a maximum of $315 \mathrm{~mm}$ wide. Each side of the central compartment was $130 \mathrm{~mm}$ long by $200 \mathrm{~mm}$ high and had a door measuring $70 \mathrm{~mm}$ by $150 \mathrm{~mm}$ cut into it to allow the 
Chapter 2: Do male and female rats learn spatial tasks differently?

subject entry to the attached arm. When assembled the overall diameter of the maze was $1.91 \mathrm{~m}$.

Figure 2.1: The radial arm maze viewed from above. Four food cups contain rewards as in the reference memory task used in the main experiment.

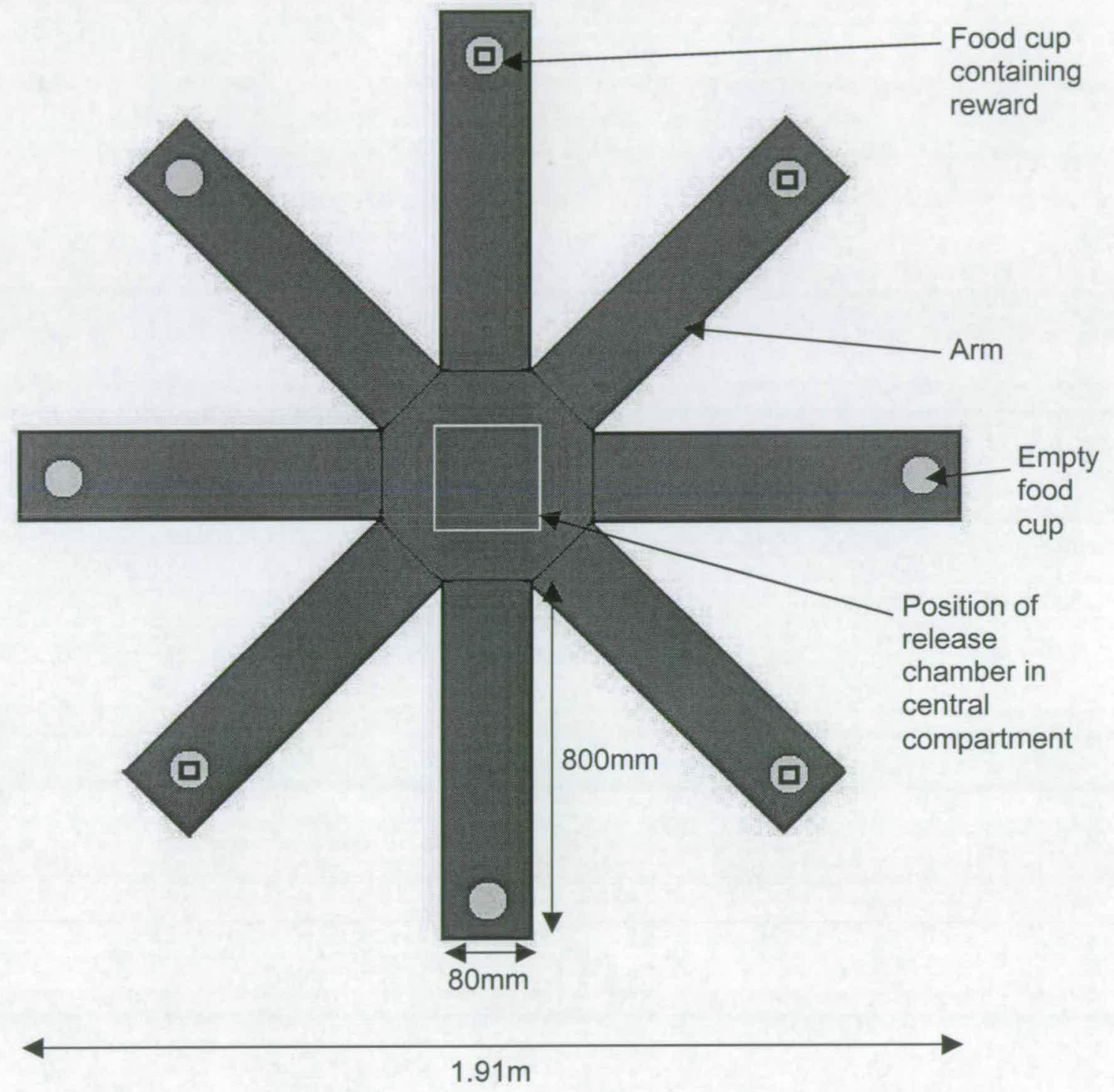


Figure 2.2: Plan of the maze room showing some of the main features. Not to scale.

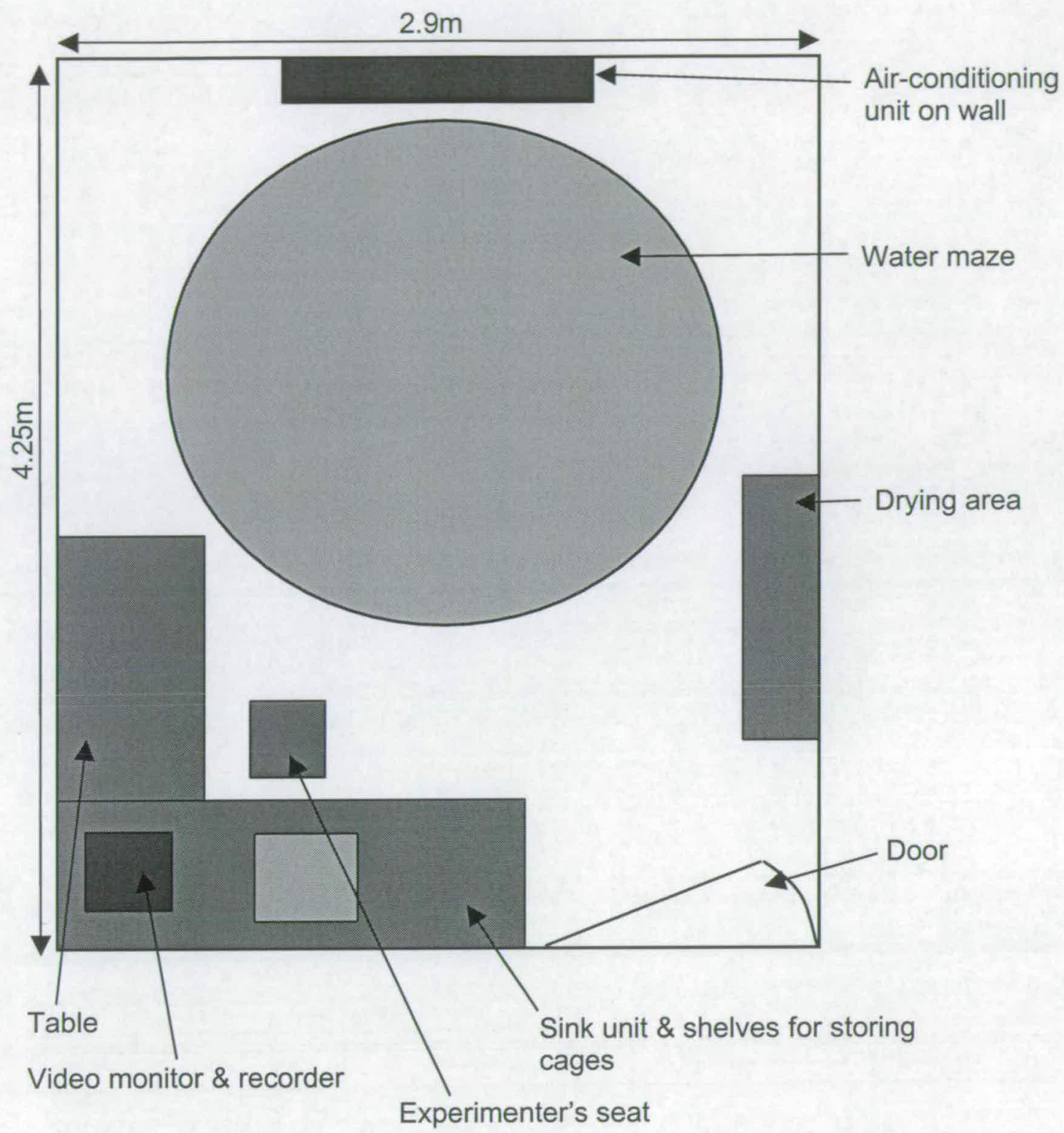

The RAM was housed in a room $(2.9 \mathrm{~m}$ by $4.25 \mathrm{~m})$ containing a water maze (figure 2.2). A radio continuously broadcasting music was located in the room. The temperature of the room was kept at $21^{\circ} \mathrm{C}$. It was a few metres away from the animal house and subjects were transported to it in their cages, which were loosely wrapped in black plastic bags and placed on a trolley. The water maze was made from white fibreglass and had a diameter of $2 \mathrm{~m}$, a depth of $650 \mathrm{~mm}$, and was raised 
$500 \mathrm{~mm}$ off the floor. The water maze contained a platform on which the RAM was placed. The floor of the RAM was $350 \mathrm{~mm}$ below the top of the water maze. The room contained many objects such as posters, an air-conditioning unit and shelves, which would have been visible to the subjects inside the maze. Two fluorescent tubes provided illumination and the one directly above the maze was dimmed by a sheet of cardboard inside the covering. A release chamber could be raised or lowered into the centre of the maze by means of a pulley. The release chamber measured $150 \mathrm{~mm}$ by $150 \mathrm{~mm}$ by $220 \mathrm{~mm}$; it was made of clear plastic and did not have a top or a bottom. A metal cup (eggcup) was placed $20 \mathrm{~mm}$ from the end of each arm, this could be filled with a food reward (a single piece of Sainsburys Honey Hoops breakfast cereal). Progress in the maze was monitored by means of a video camera above the maze and a monitor in the corner of the room; all sessions in the maze were recorded on videotape.

\section{General RAM protocol}

Each subject was given one trial in the maze per day for six days a week. The order in which the subjects went in the maze on each day was randomised. The trials started between 0930 and 1030. After the first ten subjects had been in the maze there was a break of $30 \mathrm{~min}$ before the trials continued. At the beginning of a trial the subject was placed in the release chamber for $25 \mathrm{sec}$ (timed using a stopwatch) before the chamber was raised allowing the subject free access to the maze. Some subjects developed a habit of climbing over the release chamber into the maze. Tapping their noses or shaking the release chamber discouraged this, but approximately eight. subjects persisted in this behaviour. If a subject escaped from the release chamber the chamber was lifted and the trial continued as normal. Each subject was handled for a few seconds before and after a trial to try to keep it calm. If a subject climbed on the walls it was discouraged by being gently picked up and put back in the centre of the maze. Occasionally a subject climbed out of the maze, if this happened the subject was picked up and placed back in the central compartment. No subject persistently escaped from the maze. During the trials the experimenter always wore a white lab coat and sat watching the monitor with her back to the maze. 
After all of the day's trials were completed the subjects were returned to the animal house and fed their daily ration of food. This meant that food was available to the subjects during the dark phase of the circadian cycle when they eat more (Toth \& Gardiner, 2000). The maze arms were removed from the central compartment. The floor of the entire maze was wiped with a solution of washing up liquid and each arm was randomly replaced, this prevented the subjects from learning to recognise arms by cues such as scent or scratches rather than by their position in the room. The spatial position of each arm remained constant (small red dots on the side of the water maze indicated where the arms should be) but which of the eight arms was in each position changed from day to day. It has previously been shown that rats do learn the spatial location of food rewards in the RAM rather than relying on odour cues to tell them which arms contain food (Olton \& Samuelson, 1976). The food cups were removed from the maze and washed in a solution of washing up liquid and left to dry overnight.

\section{Pretraining}

Pretraining in the radial arm maze began when the rats had stabilised at their target weights (two weeks). Most rats had 11 trials of pretraining. During pretraining all eight cups in the maze contained a reward, resulting in a working memory task designed to familiarise the rats with the procedure and the maze itself. After the release chamber was raised the subject was allowed to explore the maze for $5 \mathrm{~min}$, or until it had eaten all of the rewards. The number of rewards eaten by each subject was recorded. After eight days of pretraining the rats were freely entering the arms but many were still not eating all of the rewards. Three further days of pretraining were given where each rat was allowed to explore the fully rewarded maze for $15 \mathrm{~min}$, or until it ate all of the rewards. After this only two rats (one male and one female) were still not eating all of the rewards. These rats were given extra pretraining sessions on a day when the rest of the rats did not go in the maze. The male took two sessions and the female took three sessions until they ate all eight rewards. The main experiment was started on the following day.

\section{Experiment}


The main experiment consisted of a reference and working memory task where four out of eight arms were rewarded. Each rat was assigned an individual pattern of four rewarded arms. These were pseudorandomly generated and were different for each rat. Patterns with more than two adjacent rewarded arms, and patterns where every other arm was rewarded were excluded in order to discourage the rats from developing strategies of visiting every arm or every adjacent arm.

The rats were placed in the maze and allowed to explore until they had eaten all of the rewards. They were given one trial a day for 25 days with these patterns of rewarded arms, and then each rat was pseudorandomly assigned a different pattern of rewarded arms. The second patterns had to fulfil the same criteria as the first and also have two rewarded arms the same as in the first pattern, and two different. This meant that the second patterns of rewarded arms would be equally difficult for each subject to learn. Half of the males and half of the females had the same pattern as before, but rotated, the other subjects had totally new patterns to learn. Each subject was given a further 25 trials with the new pattern.

The number of reference memory errors (first entries into arms that never contain rewards) and the number of working memory errors (re-entries into an arm that had already been visited on that trial and did not contain a reward, either because it never did or because the reward had been eaten) were calculated for each subject per trial. These definitions take whether or not the subject ate the reward into account, for example a second entry into a rewarded arm when the reward was not eaten on the first occasion is not counted as a working memory error. However, as I did not think that the subjects would enter an arm and not eat the reward, I did not expect this to affect my results.

'Time per arm' was also calculated by dividing the total time taken to eat all of the rewards by the number of arms entered. This measure was not directly related to spatial ability, but might show sex differences. An entry into an arm was counted as such if the subject went more than $210 \mathrm{~mm}$ down the arm from the central compartment. 


\subsubsection{Results and analysis}

The data were analysed using Minitab. The first and second patterns were analysed separately. The assumptions of normality of error (Anderson-Darling normality test of the residuals) and homogeneity of residuals (plot of fitted values against residuals) were tested and appropriate transformations applied to the data. Repeated-measures analyses of variance (ANOVAs) were carried out using 'sex' as the between-subjects effect and 'block' (where each block was the mean of five trials) as the withinsubject effect. It is usual to analyse blocked data in RAM studies (e.g. Williams et al, 1990; Roof, 1993b; Seymoure et al, 1996). Although blocking did not change the significance of the sex effect, it did affect the within-subject effect by reducing variation. The following general linear model (GLM) was fitted to the data:

Response $=\operatorname{sex}+\operatorname{rat}(\operatorname{sex})+$ block + sex $*$ block

The F-ratio for the effect of sex was calculated by dividing the sex mean square (MS) by 'rat(sex)' MS in order to use an appropriate error term for the between subjects factor. When the interaction term was non-significant it was excluded from the final analysis. Working memory errors were square-root transformed for all of the analyses, neither of the other measures needed to be transformed.

\section{First pattern}

The sex by block interaction term was significant for the number of reference memory errors made (table 2.1 ). The females made significantly more reference memory errors than the males on the third block, but not on any of the other blocks (figure 2.3.a; Tukey simultaneous test $\mathrm{t}=4.183, \mathrm{p}=0.0031$ ).

The main effect of block was significant for working memory errors (table 2.1), performance tended to improve as the experiment progressed (figure 2.3.b). The effect of block was not significant for time per arm (table 2.1). The mean time spent in each arm did not change significantly as the experiment progressed (figure 2.3.c). 
Chapter 2: Do male and female rats learn spatial tasks differently?

The main effect of sex was not significant for either working memory errors or time per arm (table 2.1). However, there was a non-significant trend for males to make fewer errors than females on both of the spatial ability measures (figure 2.3.a\&b).

Table 2.1: F-ratios for the RAM repeated-measures ANOVAs. Working memory errors were square-root transformed. Non-significant (ns) block interactions $(p>0.05)$ were excluded from the analysis. Degrees of freedom for sex $=1,18$; for block $=4,76$, except when the interaction was included in the analysis when block $=4,72$ and sex block $=4,72 .{ }^{*}=p<0.1,{ }^{* *}=p<0.05,{ }^{* \star *}=p<0.01$.

\begin{tabular}{l|lll|lll} 
Response & \multicolumn{3}{|l|}{ First pattern } & \multicolumn{3}{l}{ Second pattern } \\
\cline { 2 - 7 } & sex & block & sex*block & sex & block & sex*block \\
\hline $\begin{array}{l}\text { Reference memory } \\
\text { errors }\end{array}$ & $3.76^{\star}$ & $13.17^{\star \star \star}$ & $2.61^{\star \star}$ & $<0.01$ & $2.21^{\star}$ & ns \\
$\begin{array}{l}\text { Working memory } \\
\text { errors }\end{array}$ & 2.10 & $10.03^{\star \star \star}$ & ns & 0.20 & $3.15^{* \star}$ & ns \\
Time per arm & 0.54 & 1.75 & ns & $<0.01$ & 1.97 & ns
\end{tabular}


Figure 2.3: Male and female rats' performance in the eight arm radial maze, + standard error of the mean (SEM). One block is the mean of five trials. Subjects were trained for five blocks on each of two patterns of four rewarded arms; the dashed line indicates where the pattern was changed. $N=10$ for each sex.

a) Reference memory errors (entries into arms that never contain food rewards).

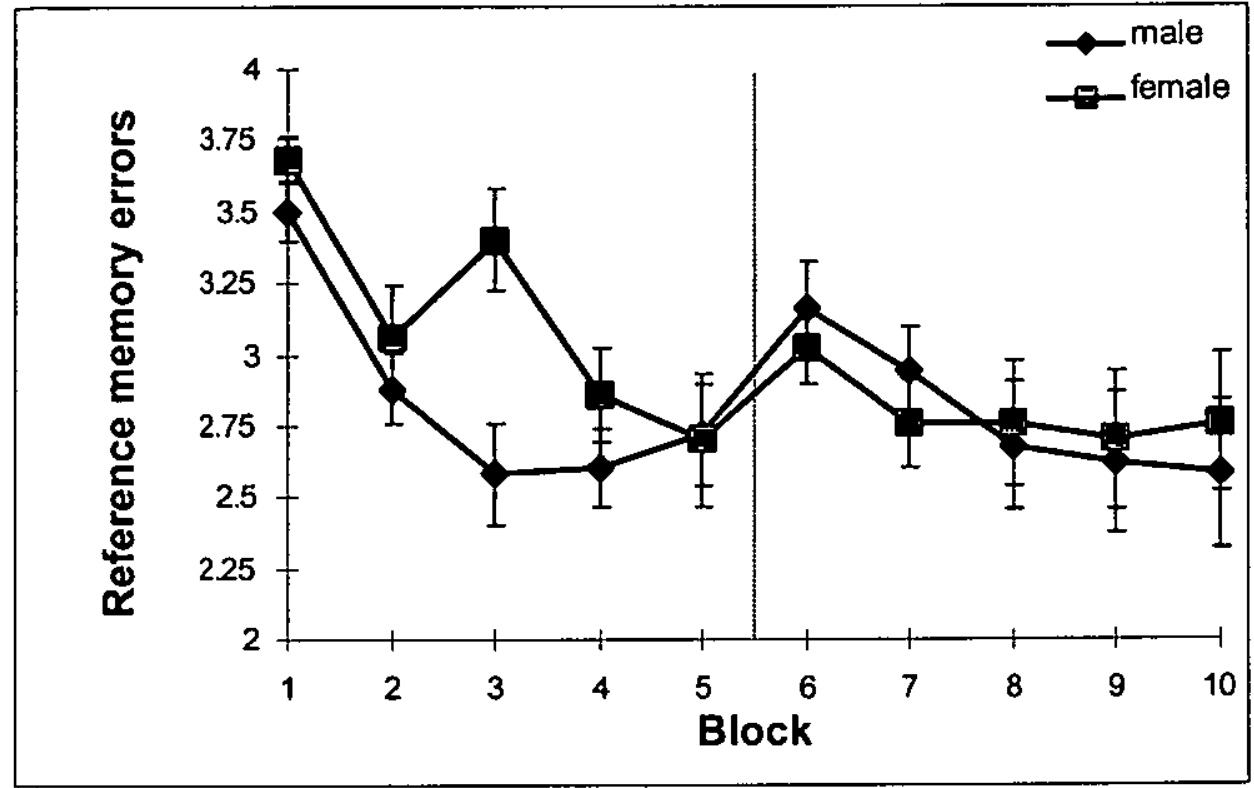

b) Working memory errors (entries into arms previously visited on that trial that did not contain a reward).

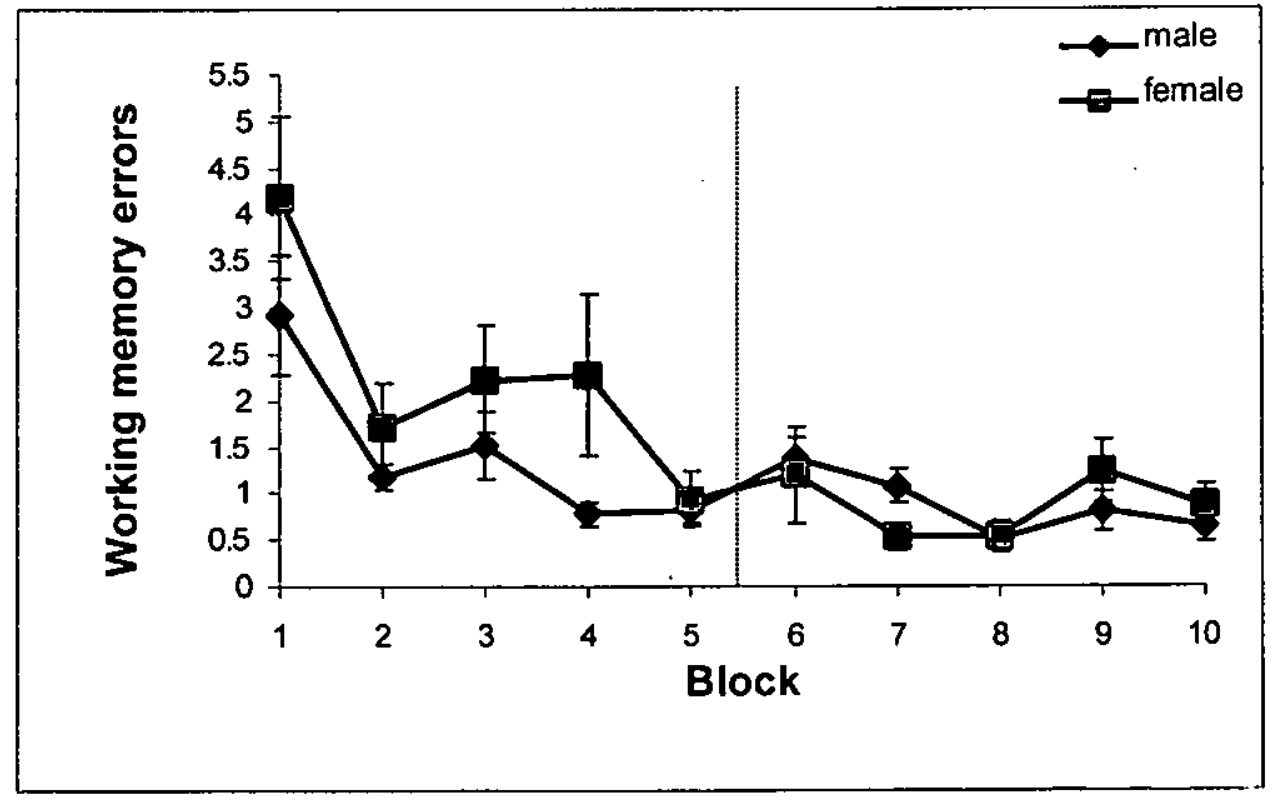


Chapter 2: Do male and female rats learn spatial tasks differently?

c) Time per arm (total time divided by number of arms entered).

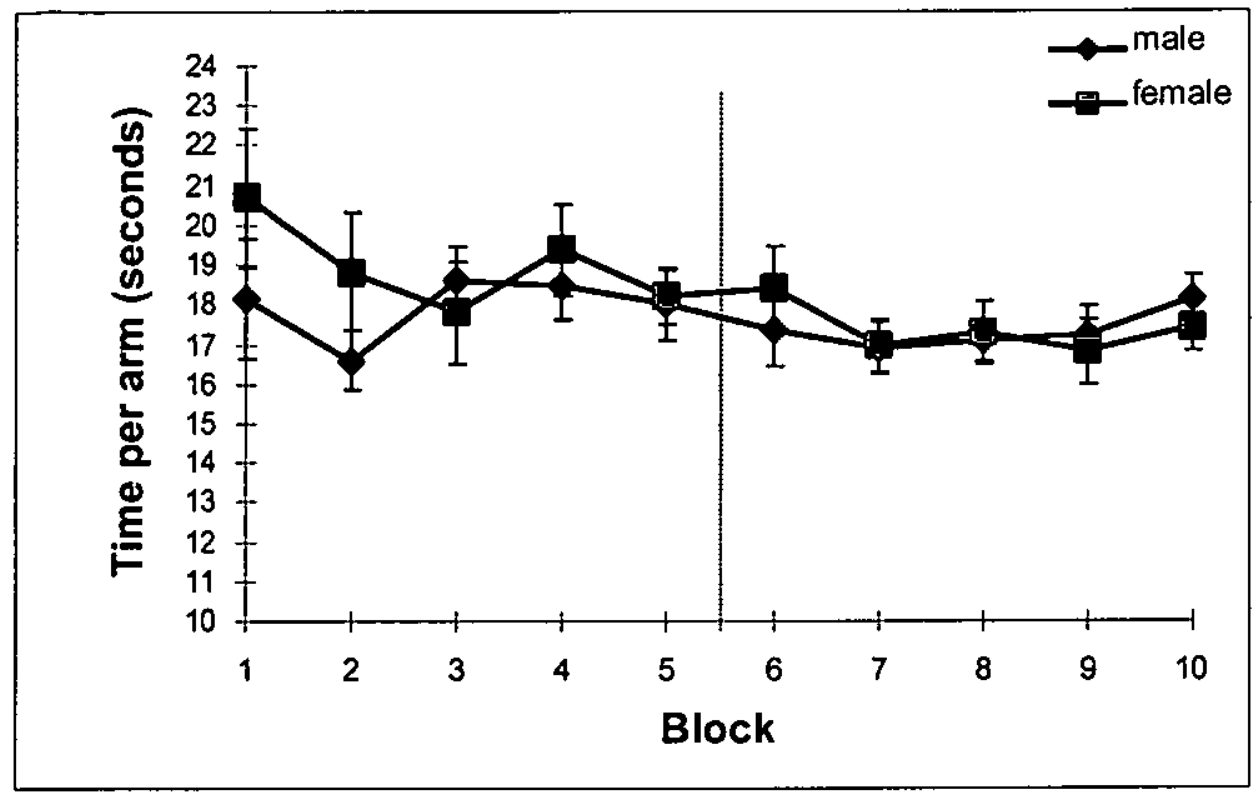

\section{Second pattern}

On the second pattern the sex by block interaction was not significant for any of the three measures. The main effect of block was significant for the number of working memory errors made, and was approaching significance for reference memory errors (table 2.1; figure 2.3.a\&b). Again, performance tended to improve with time. The time spent in each arm did not change with block (table 2.1; figure 2.3.c). There was no effect of sex on any of the measures (table 2.1; figure 2.3).

\section{Uneaten reward errors}

During the experiment it was noticed that sometimes the subjects would go down an arm that contained a reward, but fail to eat the reward. These uneaten reward errors were recorded, and analysed using a non-parametric Mann-Whitney test because the data did not meet parametric test assumptions. Females made significantly more uneaten reward errors than males during the first pattern $(w=73.0, p=0.017)$, but not during the second pattern $(\mathrm{w}=89.0, \mathrm{p}=0.241$, figure 2.4$)$. 
The significant difference between the sexes in the number of uneaten reward errors made whilst learning the first pattern could explain the trend for males to perform better than females on the spatial ability measures during the first pattern, therefore the spatial ability measures were recalculated from the raw data. This time the first entry into a rewarded arm was counted as if the reward was eaten irrespective of whether it actually was. This meant that further entries into this arm were considered to be working memory errors whether or not the arm still contained a reward. On trials where uneaten reward errors were made the overall trial length was often shortened because the trial was considered to have finished when all four rewarded arms had been visited, this meant that any working or reference memory errors made after all four rewarded arms had been visited were no longer counted.

Time per arm was not reanalysed with the uneaten reward errors removed because to do so would result in underestimating the time taken to complete the maze on the occasions that uneaten reward errors were made as time spent eating the rewards would not be included. Working and reference memory errors were analysed in the same way as before. Working memory errors were square-root transformed for both patterns.

None of the effects for working memory errors on either pattern, or for reference memory errors on the second pattern changed in significance (compare tables $2.1 \&$ 2.2). However, the trend for females to make more working memory errors during the first pattern disappeared (compare figure 2.3.b with figure 2.5.b). The main effect of sex on reference memory errors during the first pattern disappeared, but the sex by block interaction persisted (table 2.2). 
Figure 2.4: Uneaten reward errors made by male and female subjects in the radial arm maze $+/-$ SEM. One block is the mean of five trials. Subjects were trained for five blocks on each of two consecutive patterns of four rewarded arms. $N=10$ for each sex. The dashed line indicates where the pattern was changed.

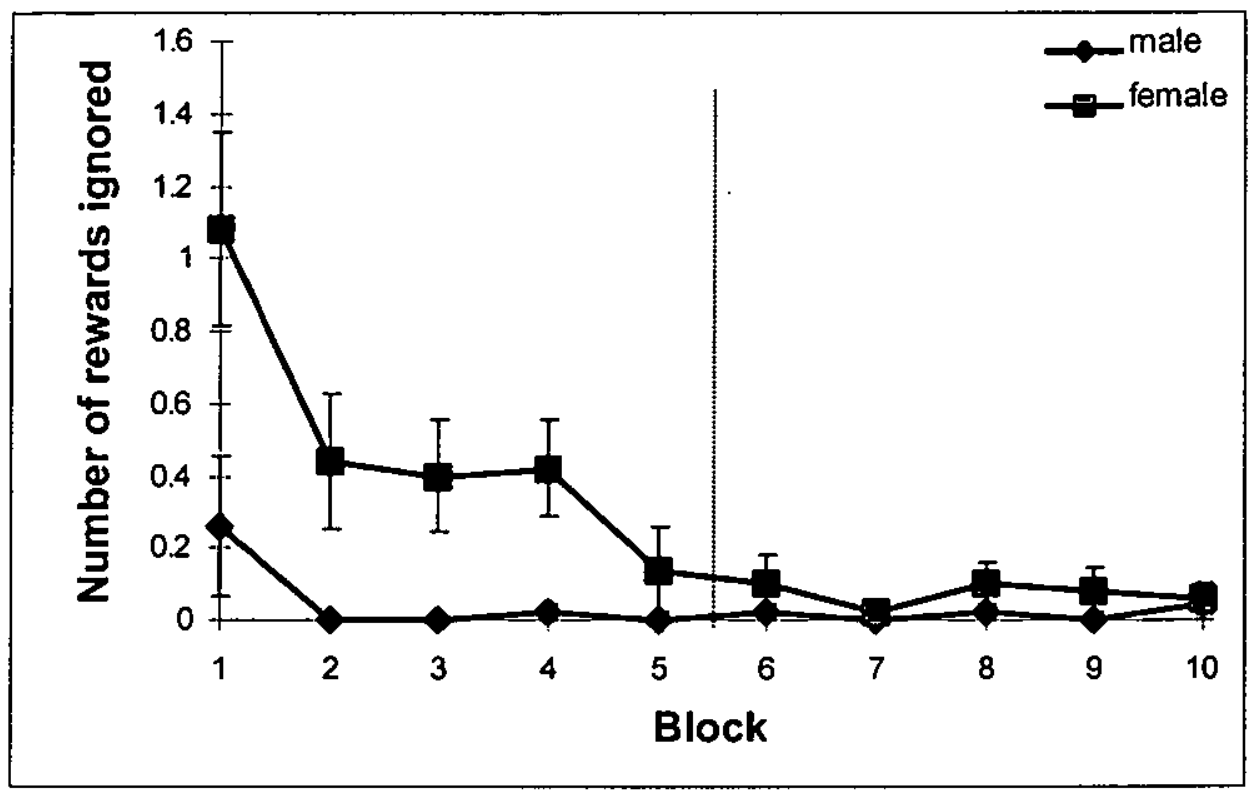

Table 2.2: F-ratios for the RAM repeated-measures ANOVAs without the uneaten reward errors. Working memory errors were square-root transformed. Nonsignificant block interactions $(p>0.05)$ were excluded from the analysis. Degrees of freedom for sex $=1,18$; for block $=4,76$, except when the interaction was included in the analysis when block $=4,72$ and sex $^{*}$ block $=4,72 .{ }^{*}=p<0.01,{ }^{* *}=p<0.05$, ${ }^{\star \star *}=p<0.01$.

\begin{tabular}{l|llll|lll}
\multirow{2}{*}{ Response } & \multicolumn{3}{|l|}{ First pattern } & \multicolumn{3}{l}{ Second pattern } \\
\cline { 2 - 7 } & sex & block & sex*block & sex & block & sex block \\
\hline Reference memory errors & 0.15 & $9.14^{\star \star \star}$ & $3.68^{\star \star}$ & 0.03 & $2.39^{\star}$ & ns \\
Working memory errors & 1.65 & $6.69^{\star \star \star}$ & ns & 0.27 & $3.05^{\star \star}$ & ns
\end{tabular}


Figure 2.5: Spatial performance excluding uneaten reward errors in the eight arm radial maze for males and females $+/$ - SEM. One block is the mean of five trials. Subjects were trained for five blocks on each of two consecutive patterns of four rewarded arms. $\mathrm{N}=10$ for each sex. The dashed line indicates where the pattern was changed.

a) Reference memory errors (entries into arms that never contain food rewards).

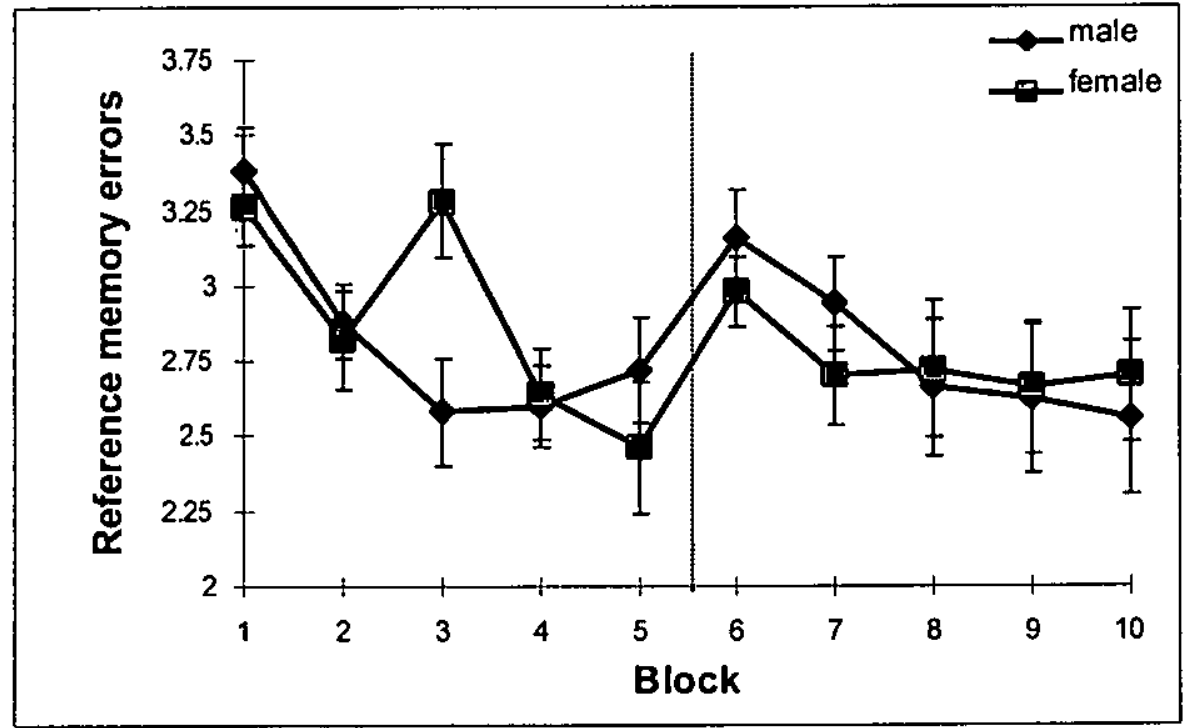

b) Working memory errors (entries into arms previously visited on that trial).

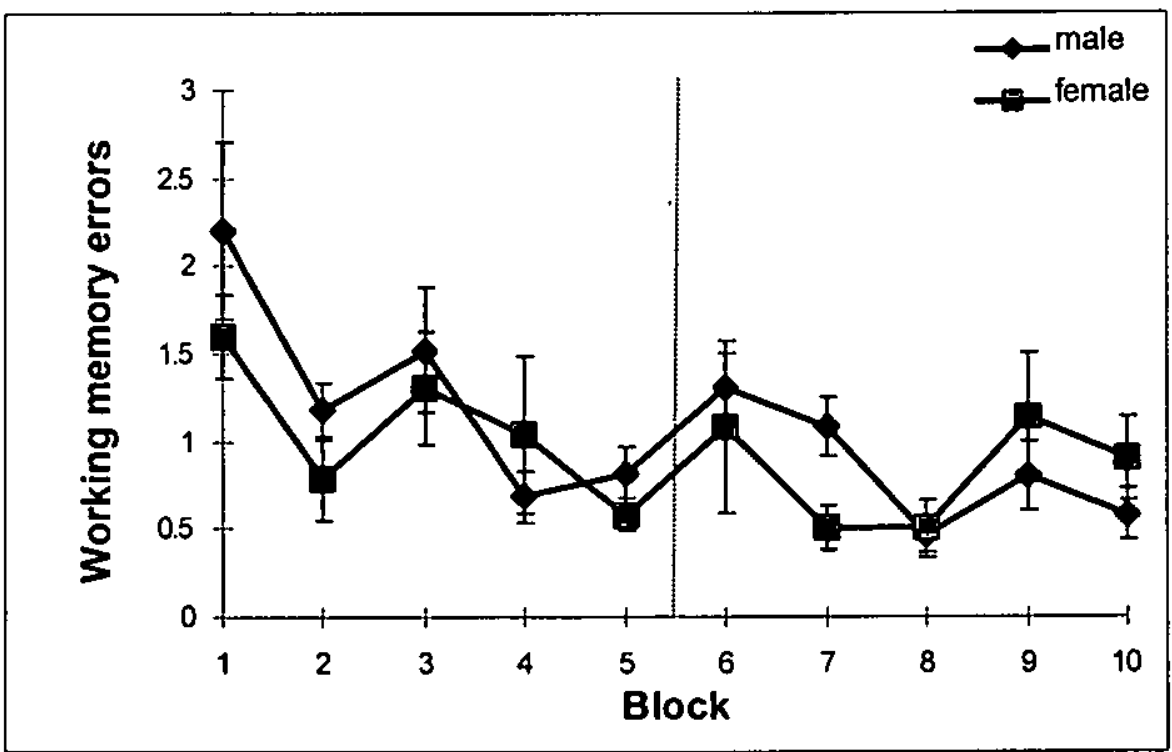




\section{Regressions}

In an attempt to determine the factors influencing the subjects' likelihood of making uneaten reward errors regressions in the form of ' $x=s e x+y$ ' were fitted to the mean number of uneaten reward errors made by each subject over both patterns using the following covariates $(\mathrm{y})$ :

1) 'Pretraining', the number of trials it took the subject to reach the criterion of eating all eight rewards within $15 \mathrm{~min}$ during pretraining. Pretraining had a significant effect on uneaten reward errors $\left(F_{1,17}=13.65, p=0.002\right)$. Subjects that took more trials to reach the criterion during pretraining made more uneaten reward errors (figure 2.6.a).

2) 'Weight gain', the total amount of weight that the subject put on during the period of food restriction for the experiment. Weight gain did not have a significant effect on uneaten reward errors which were square-root transformed for this analysis $\left(\mathrm{F}_{1,17}=0.36, \mathrm{p}=0.555\right)$.

3) 'Time per arm', the average time spent per arm for each subject during the experiment (both patterns). Time per arm had an effect approaching significance on uneaten reward errors which were square-root transformed for this analysis $\left(\mathrm{F}_{1,17}=3.71, \mathrm{p}=0.071\right)$. There was a trend for subjects that made more uneaten rewards errors to spend more time in each arm, but the effect was not very large (figure 2.6.b). 
Chapter 2: Do male and female rats learn spatial tasks differently?

Figure 2.6: Regression plots. $\mathrm{N}=10$ for each sex.

a) Mean number of uneaten reward errors made against number of trials taken to reach criterion during pretraining.

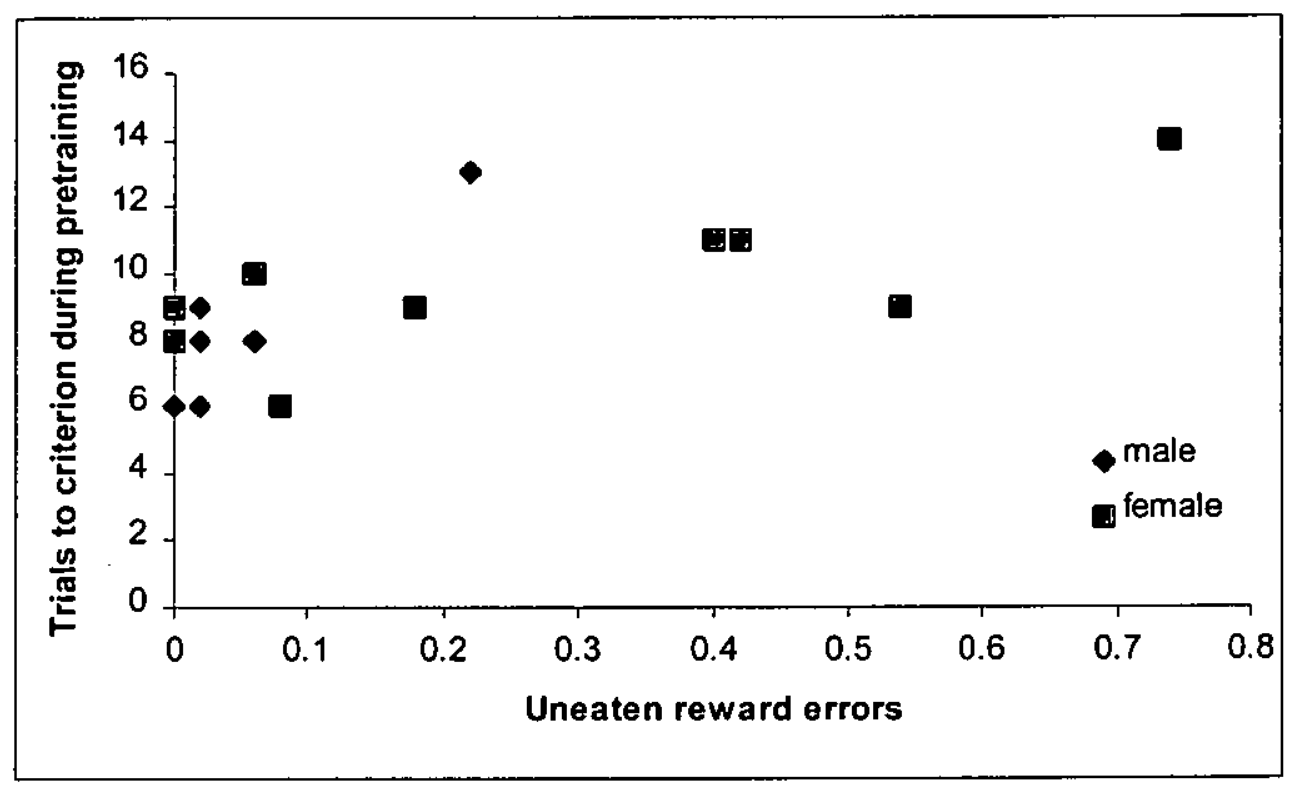

b) Mean number of uneaten reward errors made against mean time spent per arm during the main experiment.

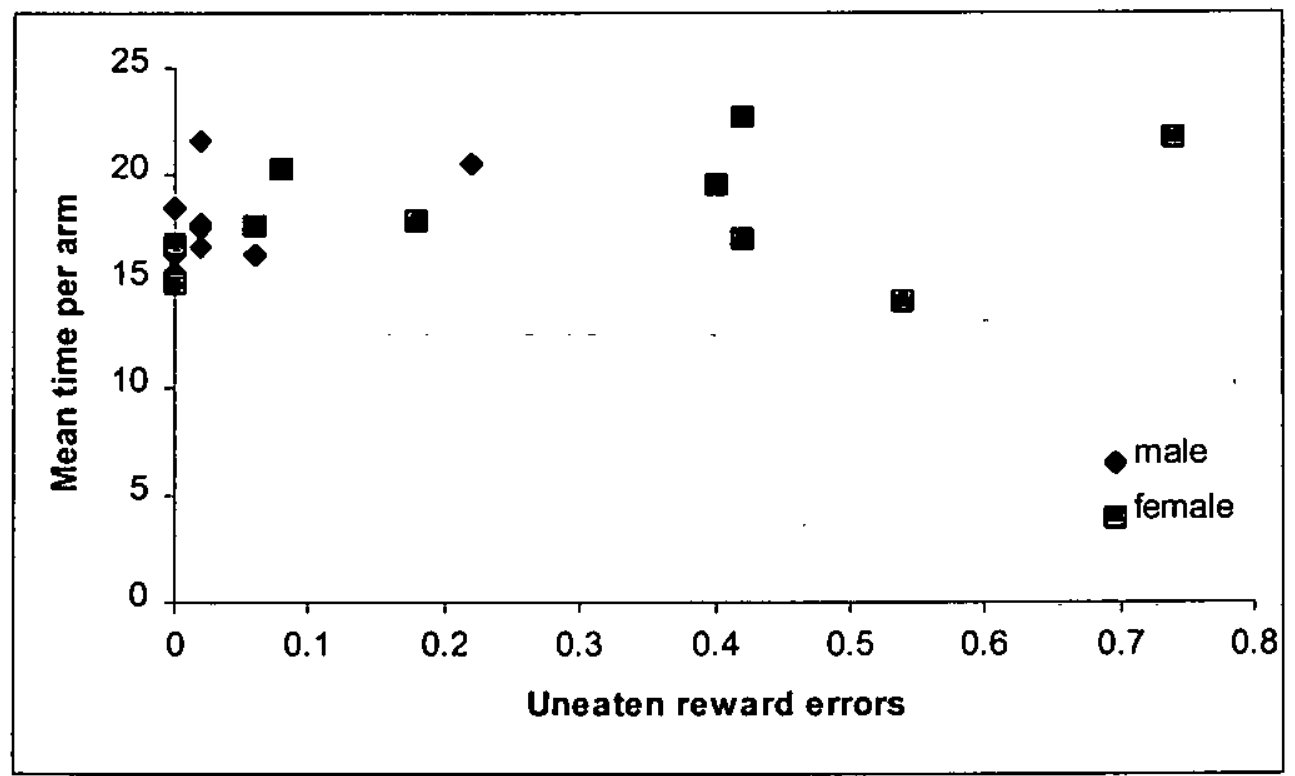




\subsubsection{Summary}

1) The male rats tended to show better spatial ability than the females whilst learning the first pattern, but not the second.

2) The mean time spent per arm entry did not differ between the sexes, nor did it change during the experiment.

3) Females were more likely than males to enter rewarded arms but fail to eat the reward. This effect was only significant during the first pattern.

4) When uneaten reward errors were removed from the data the trend towards superior male spatial ability disappeared.

5) The number of trials taken to reach criterion during pretraining was positively correlated with the number of uneaten reward errors made during the main experiment.

6) The number of uneaten reward errors made was unrelated to weight gain or the average time spent in each arm.

\subsection{Water maze experiment}

\subsubsection{Pilot study}

A MWM pilot study was carried out during May-June 2001 in order to familiarise myself with the techniques involved. Subjects were six male and six female Sprague-Dawley rats, previously used for the experiment described in chapter 4. They were approximately 20 weeks old at the start of the pilot study. All of the female rats had vaginal smears taken for 14 days prior to, and throughout, the study. Subjects were given three trials in the MWM per day for five days followed by a probe trial on the sixth day.

\subsubsection{Materials and methods}

\section{Subjects}


The main MWM experiment was carried out during June-August 2001. Subjects were eight male and 16 female young adult, experimentally naïve, Sprague-Dawley rats, weighing $175-200 \mathrm{~g}$ when supplied by B\&K Universal Ltd. All subjects had their tails marked with coloured ink to allow identification. They were housed in the animal house under the conditions described in section 2.2.2. Food and water were available ad libitum in their home cages throughout the experiment. As I intended to analyse the data from this experiment to look at the effects of the oestrous cycle on spatial ability (chapter 3 ) the female subjects had vaginal smears taken once a day from the fifth day after they arrived in the animal house until the end of the experiment. The male rats were handled for a similar period of time every day. The rats were trained on consecutive days apart from on what would have been fourth day of the first stage when I was unable to gain access to the maze room. The females were still smeared and the males handled on this day.

\section{Maze}

The water maze consisted of a $2 \mathrm{~m}$ diameter, $650 \mathrm{~mm}$ deep, white, fibreglass tub located in a small room that provided both landmark and geometric cues (described in section 2.2.2). The tub was filled with water at $21+/-1{ }^{\circ} \mathrm{C}$ and $750 \mathrm{~g}$ of coffee whitener (Sainsburys' Coffeeplus) was added to make the water opaque. A $300 \mathrm{~mm}$ high platform with a circular top area of $110 \mathrm{~mm}$ diameter was placed in the maze $350 \mathrm{~mm}$ from the side, so that it was covered by water to a depth of $25 \mathrm{~mm}$. The maze was drained at the end of every day, cleaned, and refilled the next moming.

\section{Experiment}

Each subject was pseudorandomly assigned one of two platform locations so that half of the males and half of the females had each location (east or west, figure 2.7). Using two platform positions (as opposed to one) had the advantage that it should reduce the likelihood of the subjects learning to follow odour trails to the platform (Means et al., 1992). It also allowed analysis of the whole data-set either by individual session or by block, as the mean minimum distance swam by each sex to the platform was the same irrespective of which release position was used. If only 
Chapter 2: Do male and female rats learn spatial tasks differently?

one platform position was used the fact that two of the release positions were further away from the platform position than the other two might have affected the results (figure 2.7).

Two days before training began the subjects were transported to the room that contained the water maze and left there for $3.5 \mathrm{hr}$ without swimming due to technical problems. On the day before training began each subject was allowed to swim in the maze for $1 \mathrm{~min}$ without a platform present (first probe trial). Subjects that were going to be trained with the platform in the east $(E)$ quadrant were released from the southwest (SW) release position and subjects that were going to be trained with the platform in the west (W) quadrant were released from the south-east (SE) release position. This allowed me to calculate time spent in each quadrant by untrained animals, it also allowed me to check for any quadrant biases and differences in swim speed. On the next day the water maze training began with $\mathrm{E}$ and $\mathrm{W}$ platform locations.

Figure 2.7: The Morris water maze from above, showing the platform positions used for the first training stage.

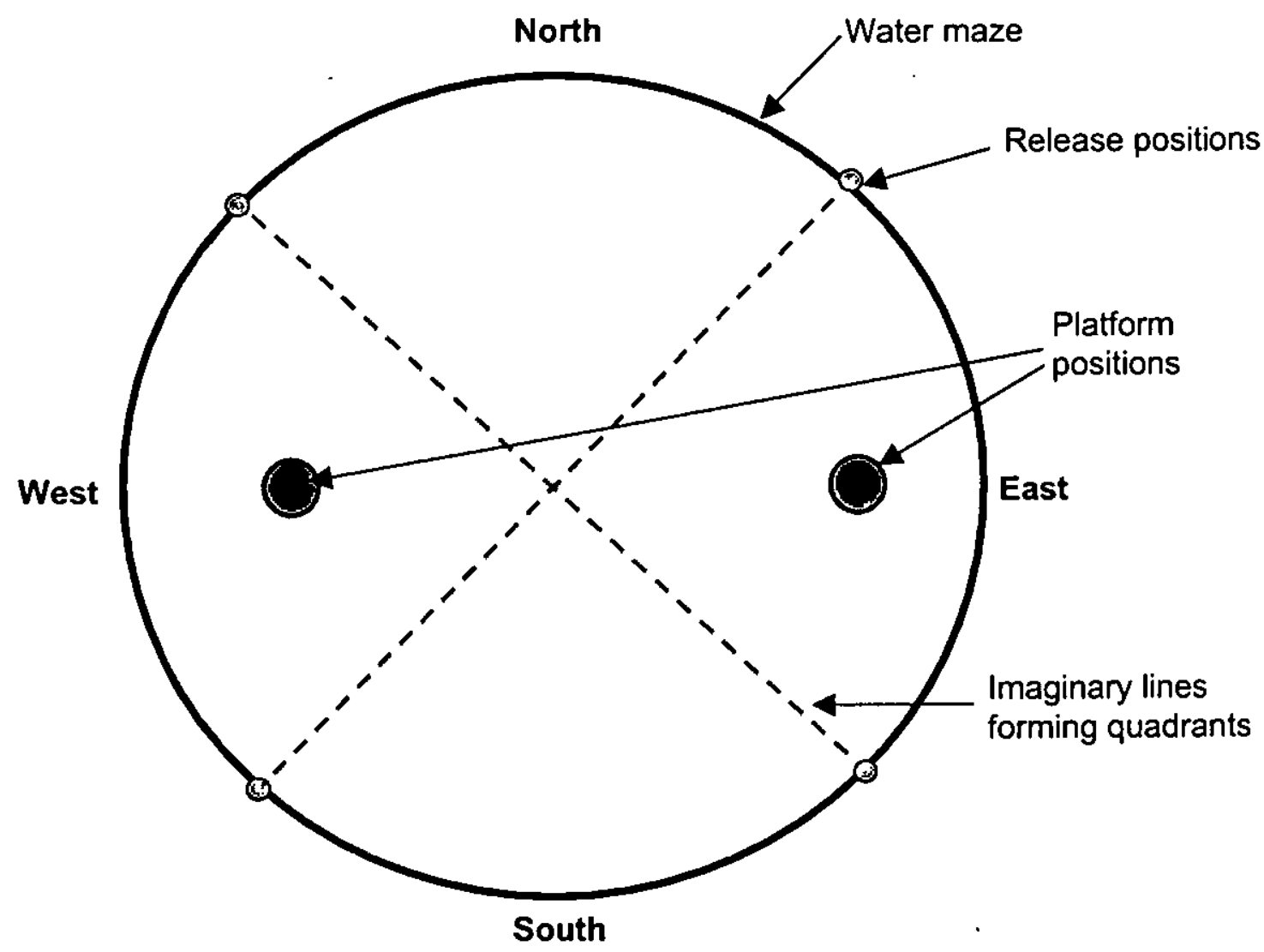


A training swim ended when the subject found and climbed on to the platform, or after $2 \mathrm{~min}$. If the rat did not find the platform within $2 \mathrm{~min}$ it was guided to the platform by the experimenter. The rat was then allowed to sit on the platform for $30 \mathrm{sec}$. Sometimes a rat jumped off the platform before the $30 \mathrm{sec}$ waiting time was finished, if this occurred it was guided back to the platform and left there for a minimum of $5 \mathrm{sec}$ before being removed from the maze. After a swim the subject was dried and placed under a heat lamp for a few minutes. Between swims faeces were removed and the water in the maze was stirred to avoid the build-up of odour trails. Each subject had two trials per day in the water maze, one in the morning session starting at approximately 1100 and one in the afternoon session, which started at about 1330. Subjects were placed in the maze in a random order within a session. There were four release positions (NE, SE, SW, NW, figure 2.7). The release position was the same for all subjects within a session, but changed between sessions so that each subject had one long swim and one short swim per day. This meant that individuals' mean scores for the day for were comparable, irrespective of platform location, because they all had the same mean minimum distance to travel in order to reach the platform. The release positions were alternated in the following order throughout the experiment: NE, SW, NW, SE, SW, NE, SE, NW. Each trial was recorded on videotape. The experimenter always wore a white coat and stood in the same place in the room in order to provide a stable landmark. Female rats, unlike males, can have their performance in the MWM disrupted if the experimenter provides an unstable landmark (Roof \& Stein, 1999).

When the subjects had reached the criterion of a mean time to find the platform for all subjects of less than $25 \mathrm{sec}$ for four consecutive trials (which took 14 trials), a second probe trial was given. However, because I needed a full day of trials to allow a mean to be taken in order to look for oestrous cycle effects, if the criterion was met on the morning trial they were still given the afternoon trial. On the day after the probe trial all subjects were pseudorandomly assigned new platform positions, either in the $\mathrm{N}$ or $\mathrm{S}$ quadrant. This was done in such a way that half of the males and half of the females had the platform in the $S$ position, also half of those that had previously been trained with the platform in $\mathrm{W}$ and half of those that had previously 
Chapter 2: Do male and female rats learn spatial tasks differently?

been trained with the platform in $\mathrm{E}$ now had the platform in $\mathrm{S}$. Trials then continued as before with the new platform positions until the learning criterion was again met (which took eight trials). Then another probe trial (probe three) was carried out. After probe three the landmarks in the room were rearranged. Posters and white sheets that could be used to provide or cover landmarks, respectively, were moved. Training then continued with each subject having the platform in the quadrant opposite to that in which it was originally trained (i.e. if originally trained with the quadrant in $\mathrm{E}$ it was now trained with the quadrant in W). Once they had again reached the criterion (which took eight trials) a fourth probe trial was carried out. Eight days after this probe trial I gave the subjects a final probe trial (probe five) to test memory retention. No smearing took place in the eight days between the fourth and fifth probe trials. The MWM training protocol is summarised in figure 2.8.

\section{Data collection}

Probe trial behaviours were recorded from the videos on two separate occasions by the same experimenter. The mean duration of three behaviours was recorded. First, time spent in the 'correct' quadrant (i.e. the most recent quadrant that the subject had been trained to find the platform in) to the nearest second. On the first probe the 'correct' quadrant was the one that would contain the platform during the first training stage. The subject was considered to be in the quadrant that the tip of its nose was in. Second, time spent in thigmotaxic behaviour, which is thought to be anxiety-related (this was defined as when the rat had the tip of its nose touching the side of the water maze; either swimming along, or scrabbling against the wall). Third, swim speed was recorded from probes one and four. This was done using a permanent marker to copy the subject's swim path between 10 and $40 \mathrm{sec}$ into the probe trial onto an acetate sheet. The length of this path was then measured using a map measurer, and the data was left in arbitrary units.

For the learning trials only the time taken to find platform to the nearest second was recorded. The subject was considered to have found the platform when both of its front feet touched it. 
Figure 2.8: Time-line showing events during the MWM experiment.

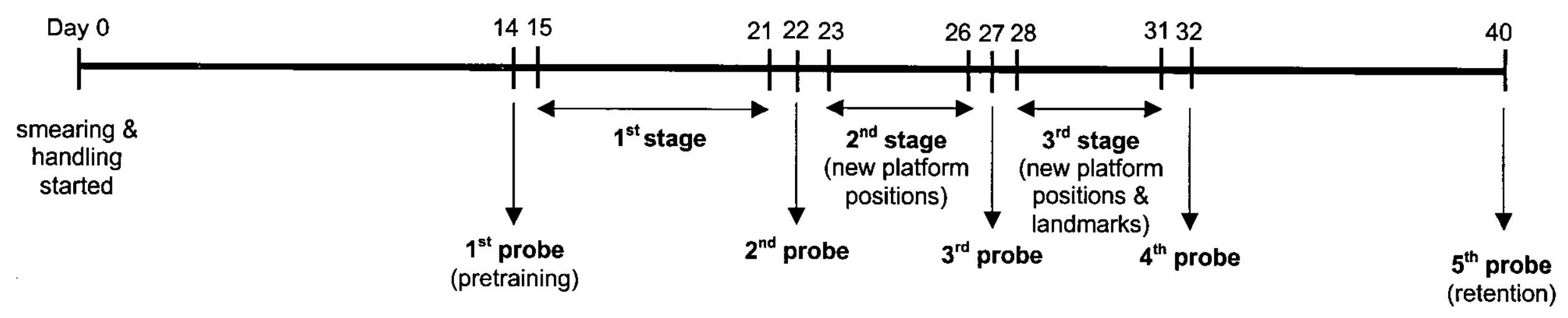




\subsubsection{Results and analysis}

Minitab was used to analyse the data. All data were checked for normality of error and homogeneity of residuals and transformations applied where appropriate.

\section{Training stages}

Repeated-measures ANOVAs were carried out on the time taken to find the platform for each of the three training stages. The first two stages were square-root transformed, the third was $\log 10$ transformed. The trials were blocked by taking the mean of the morning and afternoon trials for each day for each subject, in order to remove any time of day effects. One between-subjects effect (sex) and one withinsubject effect (day) were included in the analyses. The following model was used:

Time to platform $=\operatorname{sex}+\operatorname{rat}(\operatorname{sex})+$ day $+\operatorname{sex} *$ day

The F-ratio for the effect of sex was calculated by dividing sex MS by 'rat(sex)' MS so that an appropriate error term was used for the between subjects factor.

None of the interaction terms were significant and therefore they were removed from the final model. The within subject effect of day was significant for all three stages due to the rats finding the platform faster as training progressed (figure 2.9; stage 1, $F_{6,138}=12.73, p<0.001 ;$ stage 2, $F_{3,69}=13.01, p<0.001 ;$ stage 3, $\left.F_{3,69}=15.58, p<0.001\right)$. None of the effects of sex were significant (stage $1, F_{1,22}=2.00, p=0.172$; stage 2, $\mathrm{F}_{1,22}=0.12, \mathrm{p}=0.735$; stage $3, \mathrm{~F}_{1,22}=0.91, \mathrm{p}=0.351$ ). 
Figure 2.9: Time taken to find the hidden platform in the Morris water maze by sex $+/$ - SEM. Each day is the mean of two trials, female $n=16$. male $n=8$.

a) First training stage.

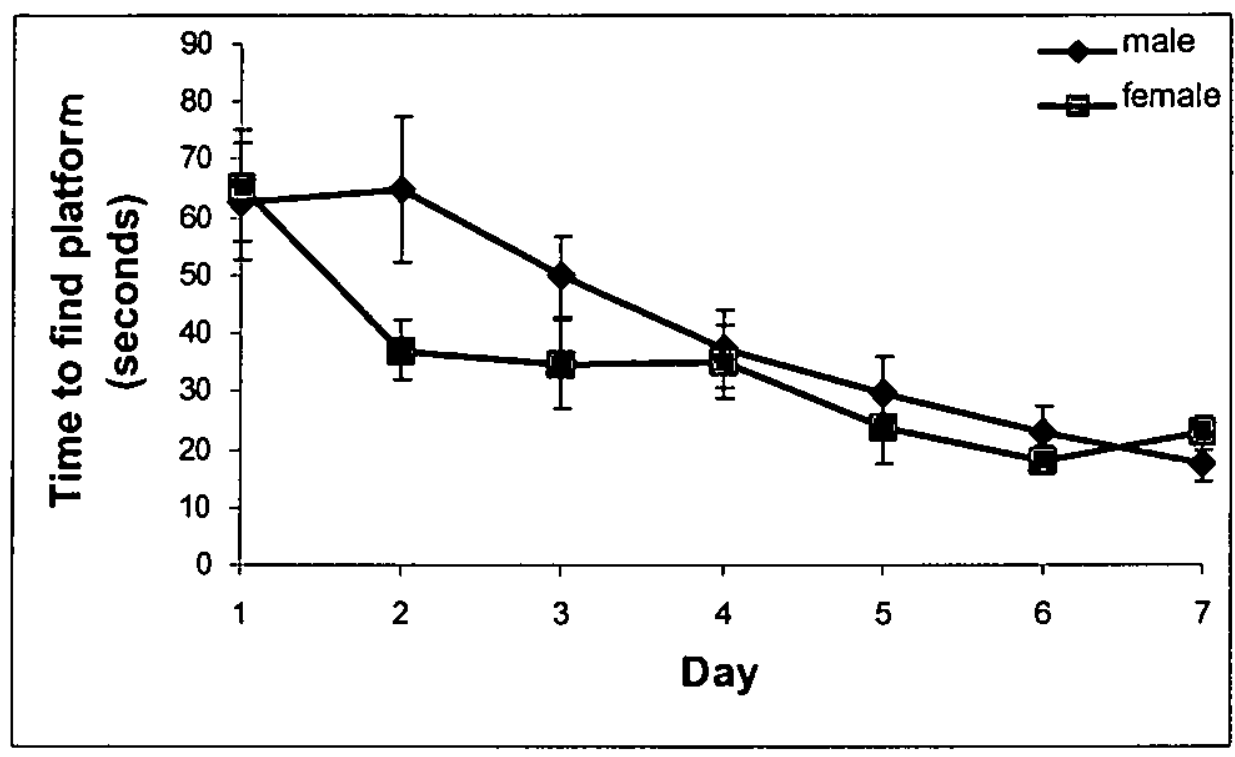

b) Second training stage, new platform positions.

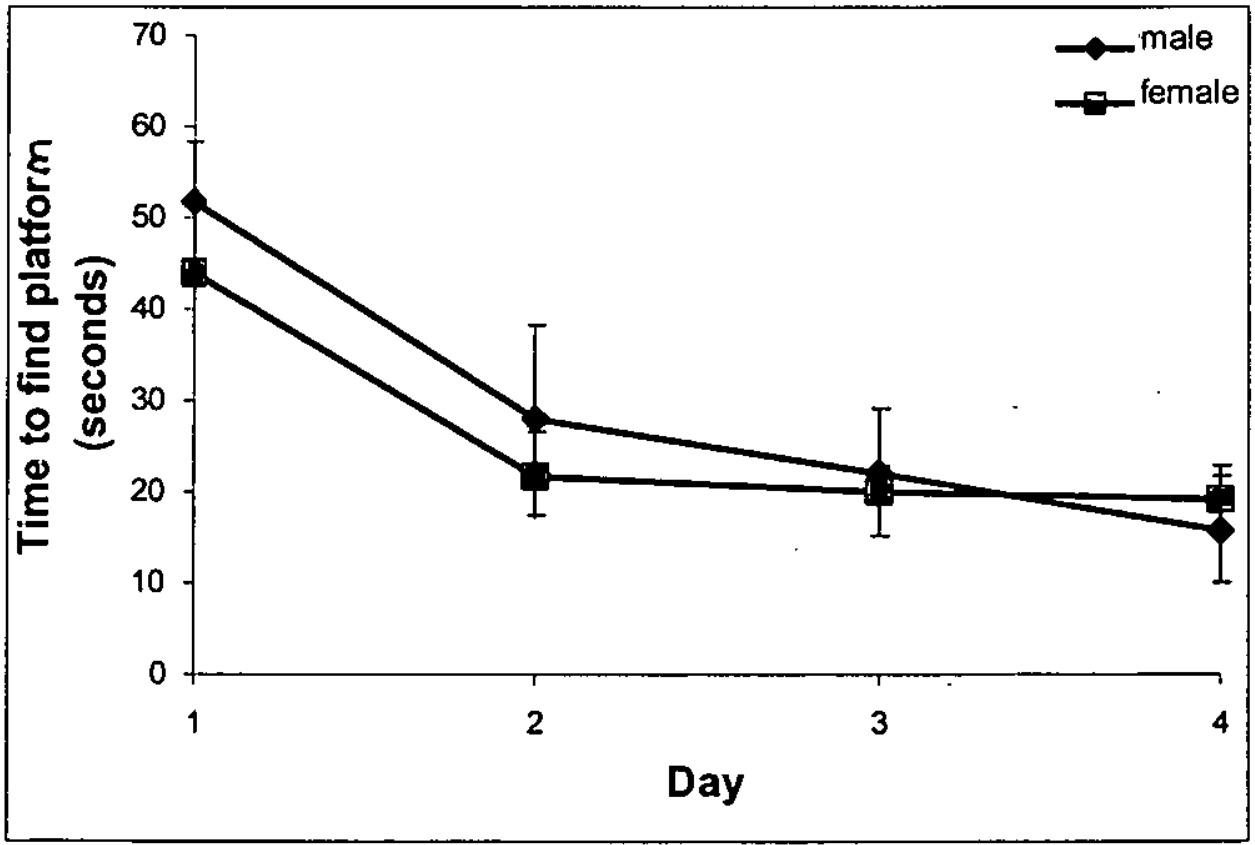


Chapter 2: Do male and female rats learn spatial tasks differently?

c) Third training stage, new platform positions and altered landmarks.

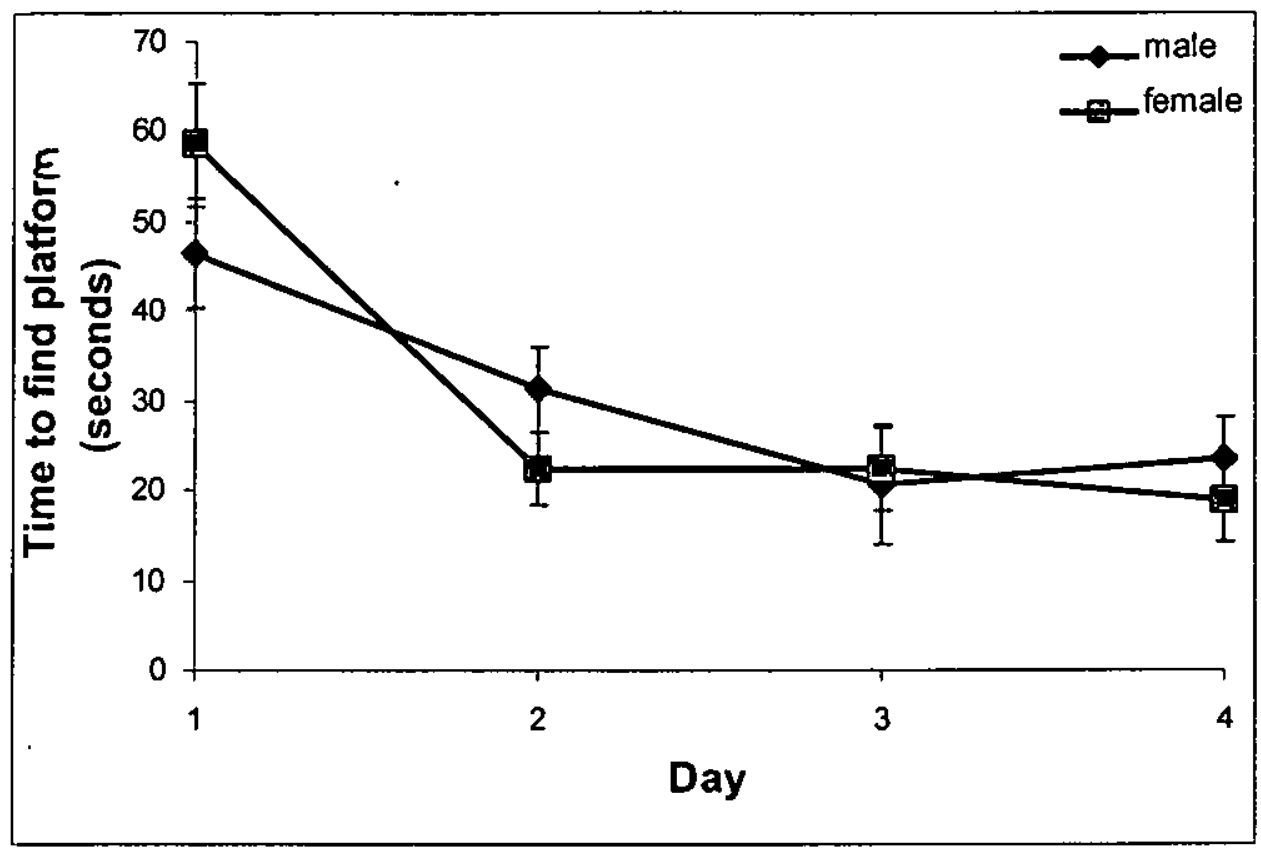

\section{Probe trials}

a) Time in correct quadrant

The time spent in the correct quadrant during the five probe trials was analysed to check for learning (or quadrant biases in the case of probe 1). This was done using a one-sample t-test to test for significant differences between the actual time and the random expectation of $15 \mathrm{sec}$ (if the subject was entering all four quadrants at random during the $60 \mathrm{sec}$ probe trial).

These tests show that subjects spent a significantly different amount of time to the random expectation of $15 \mathrm{sec}$ in the correct quadrant during probes one, two, three and four, but not during probe five (figure 2.10; one $t=-4.36, p=0.0002$; two $t=6.19$, $\mathrm{p}<0.0001$; three $\mathrm{t}=5.68, \mathrm{p}<0.0001$; four $\mathrm{t}=6.04, \mathrm{p}<0.0001$; five $\mathrm{t}=1.75, \mathrm{p}<0.094$ ). In probe one (before any training) they spent less time than expected in the 'correct' quadrant. In probes two, three and four they spent more time than expected in the correct quadrant due to having learnt where the platform was. By probe five (the 
Chapter 2: Do male and female rats learn spatial tasks differently?

retention probe, 9 days after training finished) they appeared to have forgotten where the platform had been located during the final training stage.

The GLM model 'time=sex', equivalent to a two-sample t-test, was used to test for the effect of sex on the time spent in the correct quadrant during each of the probe trials. The first probe data were square-root transformed. The sex term was not significant for any of the five probe trials (figure 2.10; probe $1, \mathrm{~F}_{1,22}=1.18, \mathrm{p}=0.290$; probe $2, \mathrm{~F}_{1,22}=0.35, \mathrm{p}=0.562$; probe $3, \mathrm{~F}_{1,22}=0.93, \mathrm{p}=0.344$; probe $4, \mathrm{~F}_{1,22}=0.53$, $\mathrm{p}=0.473$; probe $\left.5, \mathrm{~F}_{1,22}=0.19, \mathrm{p}=0.669\right)$.

Figure 2.10: Time spent in the quadrant that had previously contained the platform ('correct' quadrant) during the 60 sec probe trials $+/$ - SEM, females $n=16$, males $n=8$. Dashed line shows random expectation of $15 \mathrm{sec}$.

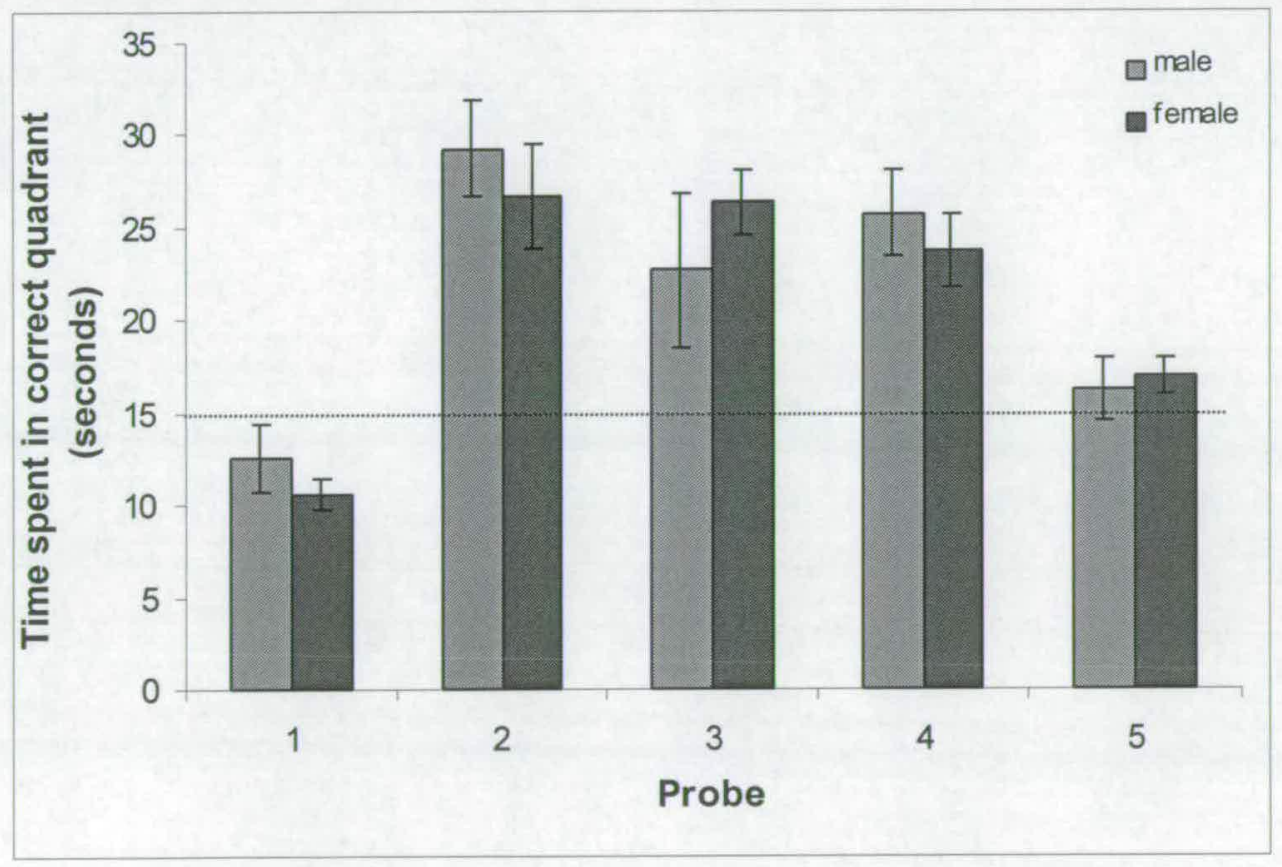

In order to investigate why the subjects were spending less time than expected in the 'correct' quadrant during the first probe, time spent in each half of the maze relative to the release position was recorded for each subject. The two maze halves were called 'near' and 'far', where near was the time spent in the two quadrants closest to 
Chapter 2: Do male and female rats learn spatial tasks differently?

the release position (south and east for those subjects released from SE, and south and west for those subjects released from SW) and far was the time spent in those quadrants opposite to the release position. The near time plus the far time should equal $60 \mathrm{sec}$, but due to recording error this was not always so. This problem was overcome by assuming that the relative proportion of time spent in each half was correct and creating standardised times by using the following equation:

Standardised time $=($ actual time/total time $) * 60$

Where 'actual time' was the recorded time for each half for each subject, and 'total time' was the sum of the near and far times for each subject.

A one-sample t-test was used to test for a departure from the random expectation of $30 \mathrm{sec}$, this was significant $(\mathrm{t}=10.14, \mathrm{p}<0.0001)$. The subjects spent more time in the half of the water maze closest to the release position, and less time in the far half than expected by chance (figure 2.11). A two-sample t-test was used to test for an effect of sex on the data, which was approaching significance $\left(\mathrm{F}_{1,22}=3.23, \mathrm{p}=0.086\right)$, females tended to spend more time in the quadrant close to the release position than males (figure 2.11).

Figure 2.11: Time spent in the near and far halves of the water maze relative to the release position during the first probe trial $+/$ - SEM, females $n=16$, males $n=8$. Dashed line shows random expectation of $30 \mathrm{sec}$ in each half.

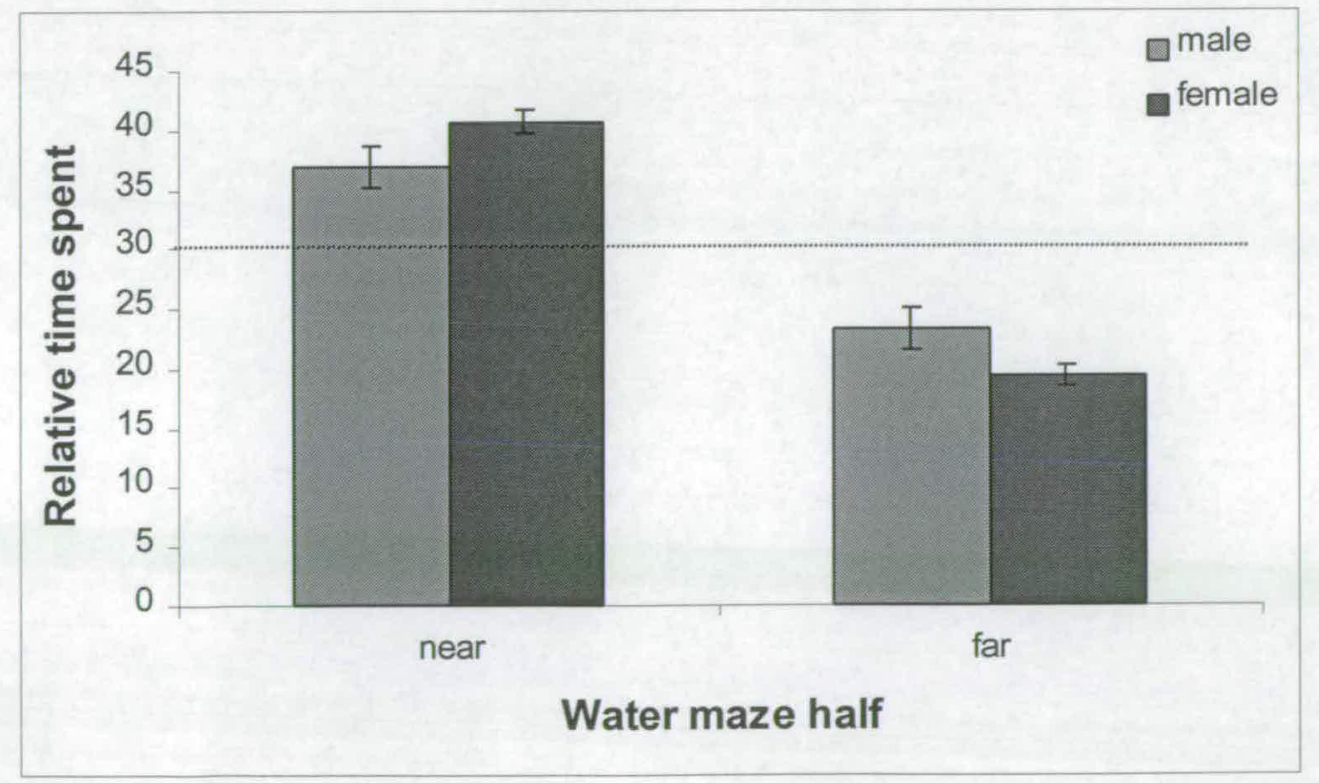




\section{b) Thigmotaxis}

Time spent in thigmotaxic behaviour during each probe trial was analysed using a repeated-measures ANOVA (GLM 'time $=\operatorname{sex}+\operatorname{rat}(\operatorname{sex})+$ probe $=s e x *$ probe') with one within-subject factor (probe) and one between-subjects factor (sex). The interaction term was not significant. The main effect of probe was significant $\left(\mathrm{F}_{4,92}=154.71, \mathrm{p}<0.001\right)$, rats spent more time in thigmotaxic behaviour during the first probe than the others (figure 2.12). The main effect of sex was not significant $\left(\mathrm{F}_{1,22}=0.01, \mathrm{p}=0.918\right)$.

Figure 2.12: Time spent in thigmotaxic behaviour during the 60 sec probe trials $+/-$ SEM, females $n=16$, males $n=8$.

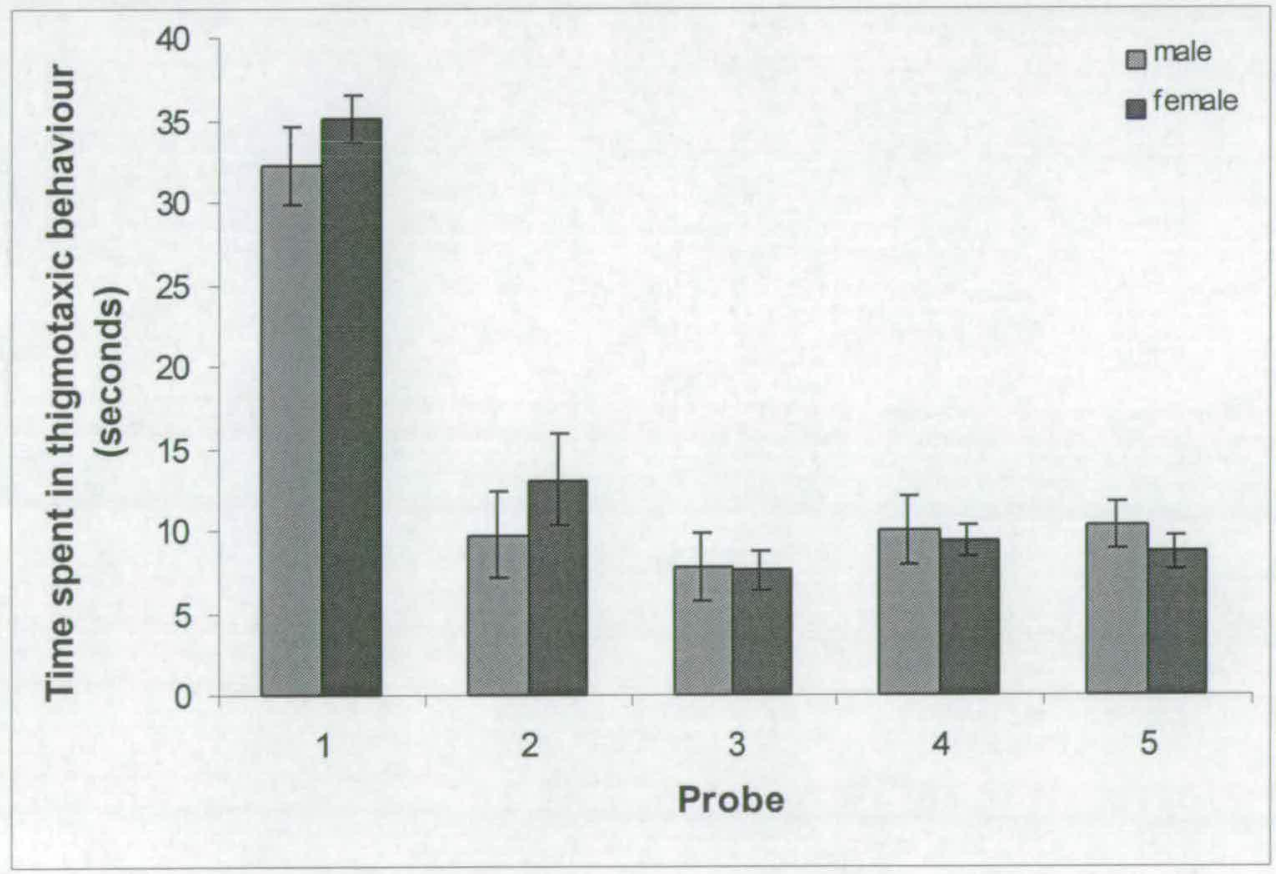

c) Swim speed

Swim speed on probes 1 and 4 was analysed to test whether there were differences in male and female swim speed, and whether swim speed changed during the experiment. A repeated-measures ANOVA (GLM 'swim speed $=\operatorname{sex}+\operatorname{rat}(\operatorname{sex})+$ 
probe + sex*probe') was used. The interaction term was not significant, neither were either of the main effects ( $\operatorname{sex} \mathrm{F}_{1,22}<0.01, \mathrm{p}=0.981$; probe $\mathrm{F}_{1,23}=1.07, \mathrm{p}=0.311$ ). There were no differences in swim speed between the sexes, neither was there a significant change in swim speed from probe 1 to probe 4 (figure 2.13).

Figure 2.13: Swim speed (distance travelled in arbitrary units between 10 and 40 sec into the probe trial) during probe trials $+/$ - SEM, females $n=16$, males $n=8$.

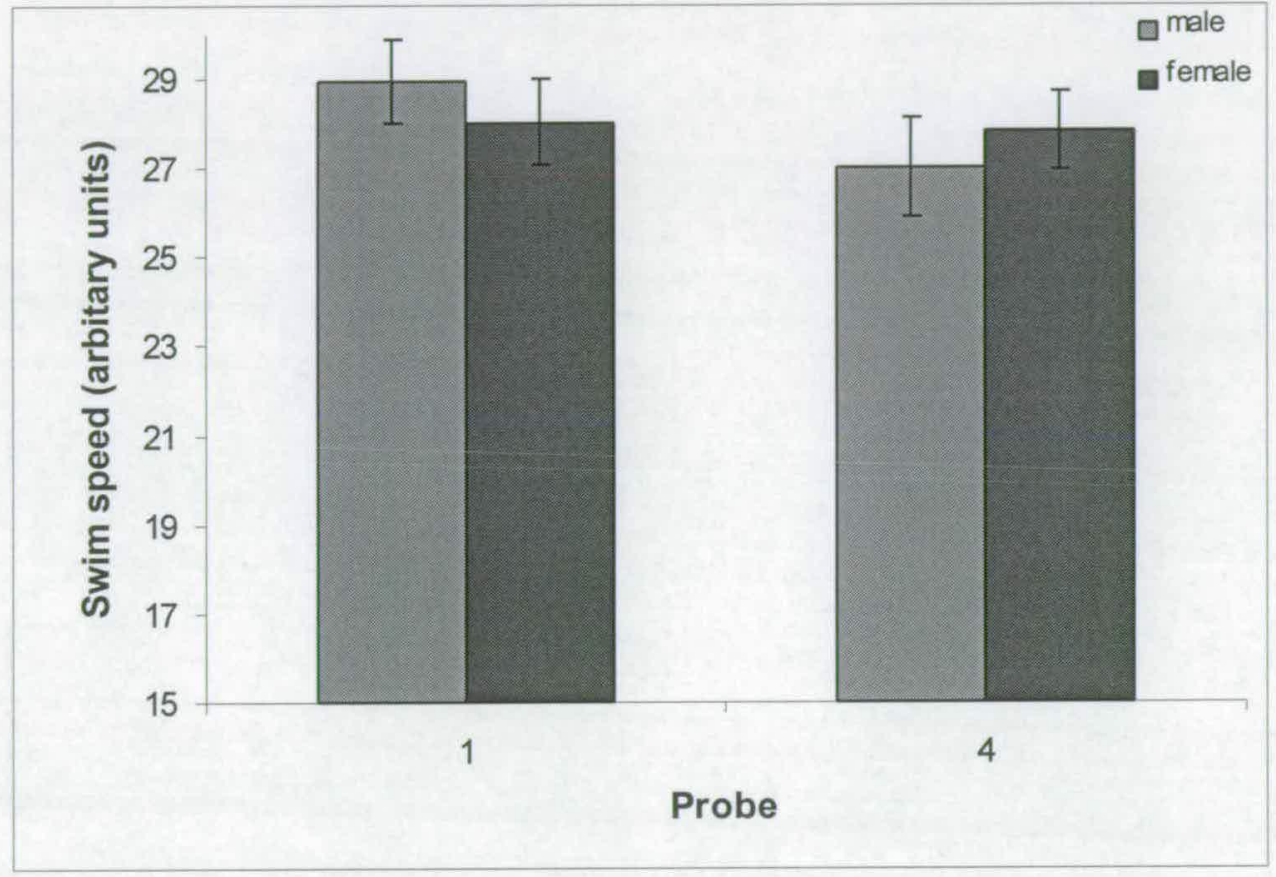

\subsubsection{Summary}

1) There were no significant effects of sex on the spatial ability of laboratory rats in the water maze.

2) The sexes did not differ significantly in the amount of thigmotaxic behaviour displayed, or in swim speed in the water maze.

3) The subjects forgot the location of the platform within eight days of their last training session. 
Chapter 2: Do male and female rats learn spatial tasks differently?

4) During the first probe trial the subjects spent significantly more time in the half of the maze near to the release position than in the far half.

\subsection{Discussion}

In summary:

1. Radial arm maze

a) First pattern: Overall, the females tended to make more reference memory errors than the males on the first pattern of the RAM task, but this effect disappeared when uneaten reward errors were taken into account. The females made more reference memory errors than the males on the third block of the RAM first pattern even when uneaten reward errors were taken into account; but as I was specifically looking for sex differences at the beginning of the stages, and this result was not replicated in the MWM experiment, I conclude that it was due to experimental variation rather than an actual sex difference in spatial ability. There were no sex differences in working memory;

b) Second pattern: There were no sex differences in either working or reference memory.

2 Morris water maze

There were no sex differences in spatial ability at any of the three stages of the water maze task.

To conclude, there were no overall sex differences in spatial ability. I had originally hypothesised that the stage of the tasks at which sex differences were found would provide information about the part of learning a spatial task in which sex differences occur. Namely, whether there are sex differences in learning the maze procedure, the rewarded positions, or the extra-maze cues. As sex differences in spatial ability were not found at any stage of either task I am unable to address these specific hypotheses. 
However, there was a sex difference in uneaten reward errors, which females made more frequently than males during the first pattern of training in the RAM. Although the RAM is a commonly used test of spatial ability, uneaten reward errors are rarely mentioned in the literature. Olton and Samuelson (1976), who invented the RAM, mention in passing that at the beginning of an experiment the subjects sometimes did not eat food rewards, even though they appeared to have noticed them. Similarly, Katoh et al. (1992) using the RAM to test gerbils, briefly mention "no-eating" (defined as "entries into the arms, in which there was a (food) pellet, without eating") and that the frequency of such behaviour decreased to about zero after four trials.

Boakes et al. (2000) analysed the number of uneaten reward errors made in an eight arm RAM with four rewarded arms by sex and strain (hooded Wistar versus albino Wistar rats). They found that these errors were more likely to be made by the hooded rats than the albinos. The hooded females tended to make more such errors than the males, but there were no sex differences within the albino rats. Boakes et al. (2000) describe uneaten reward errors as being due to 'exploration' motivation conflicting with the food incentive that is usually assumed to be driving subjects' behaviour within a RAM. The observed pattern of uneaten reward errors can therefore be explained by exploralion motivation exceeding 'food' motivation during the first few trials in the RAM. However, exploration motivation is reduced as the maze becomes familiar causing the frequency of uneaten reward errors to likewise fall as the food motivation prevails. This theory can also explain the sex differences in uneaten reward errors as female rats show more exploratory behaviour than males (a fact that has previously been suggested as a possible explanation for females' poor maze learning performance by Joseph et al., 1978).

The decreasing-with-familiarity pattern seen in the uneaten reward errors is typical of behaviours related to stress associated with novelty. Therefore, sex differences in stress response are a potential explanation for the observed sex differences in these errors. A second possible explanation is that, rather than the females being more exploration motivated, the males were more food motivated, and this caused them to eat more of the rewards that they encountered. As a partial test of this theory uneaten 
reward errors were tested for correlation with weight gain allowing for sex. Heavier rats within each sex might be more satiated, and therefore less motivated by food and more likely to make uneaten reward errors, than their lighter counterparts. However, uneaten reward errors were not correlated with weight gain and the subjects that made more uneaten reward errors were not those that gained more weight. This suggests that the tendency to make uneaten reward errors may not be related to food motivation.

Another question that must be addressed is whether the uneaten reward errors could be the cause of sex differences in spatial ability in other RAM studies. This seems to be a plausible interpretation, providing that spatial performance in the maze is calculated using 'entry into a rewarded arm and eating the reward' as the criterion for a correct choice rather than simply 'entry into a rewarded arm'. Very few studies explicitly state the criterion used to assess a correct choice (Juraska et al., 1984 and Luine et al., 1994 are among the few that do). However, this seems to be an unlikely explanation for most of the studies that find sex differences in spatial ability in rats because uneaten reward errors are specific to the RAM and sex differences in rats have also been found using other tests of spatial ability (e.g. Barrett \& Ray, 1970; Krasnoff \& Weston, 1976; Isgor \& Sengelaub, 1998; Cimadevilla et al., 1999).

There were some indications from the MWM experiment that female rats were more stressed by the maze procedure than males. On the first probe trial females tended to spend more time in the half of the maze nearest to the point that they were released from than the males (figure 2.10). This could be described as anxiety or stress related behaviour. Similarly, although there were no significant differences between the sexes in thigmotaxic behaviour (wall-hugging, which is associated with stress) on the probe trials, there was a trend across the first two probe trials for females to show more thigmotaxic behaviour than males (figure 2.12). This trend is not apparent in the later probe trials, and could be explained by the females experiencing more stress than males due to the novelty of the task. It is possible that the extensive handling received by the subjects due to smearing prior to the start of the MWM training may 
have eliminated any significant effects of stress, as handling is known to reduce stress in rats (Manser, 1992).

Stress has been shown to have sex-specific effects on many different types of learning (Shors, 1998). In spatial tasks stress-reducing measures have been shown to reduce sex differences. For example, pretraining in the MWM, which has been hypothesised to reduce the stress associated with the task, prevents sex differences from being found in rats (Perrot-Sinal et al, 1996). Some studies have also manipulated levels of opiate activity, as increased endogenous opiate activity is associated with stress. When opiate activity is inhibited the performance of female meadow voles and deer mice in the MWM is improved, and sex differences are .reduced (Galea et al., 1994c; Kavaliers et al., 1996). This could be because female rodents are more affected by spatial-ability-impairing opiates caused by stress, than males.

If female rodents are more stressed than males by maze tasks and this does impair their apparent spatial ability the effect would be likely to be especially noticeable in the early stages of testing when the task is novel. It is also possible that stress suppresses appetite (Miller \& Dess, 1996), if so this could explain why the apparent sex differences observed in the RAM were due to the females not eating the rewards.

A further question raised by the MWM experiment is why disrupting the landmarks did not differentially affect the sexes' spatial ability as had been predicted. Male and female rats tend to learn different aspects of their spatial environment; females have been shown to use local visual cues to solve the MWM, while males use navigational (distant) cues (Kanit et al, 1998a; Roof \& Stein, 1999). Williams et al. (1990) found that female rats used both landmark (movable objects in the room) and geometrical (the shape of the room) cues to solve a RAM, while male rats used only geometrical room cues. In my experiment, neither sex seemed to be very disrupted when the landmarks were disturbed (compare figure 2.9.c to figure 2.9.b where only the platform position was altered). It may be that the landmarks were not sufficiently disrupted, or that the specific landmarks that the subjects were paying attention to were not disrupted. 
The fact that the rats' performance in the RAM was disrupted by changing the pattern of rewarded arms (figure 2.5) suggests that they were using spatial information to solve the maze rather than odour cues from the rewards. This provides further support for Olton and Samuelson's (1976) findings (discussed in section 2.2.2).

It is possible that the measures used to quantify spatial ability in my two tasks were not appropriate. There are several other ways in which spatial ability may be measured in the RAM; these include the total number of arms entered until all the rewards have been found, and the number of correct entries made during the first four entries. Both of these measures include both working and reference memory errors and I did not report them because I thought that using working and reference memory errors would provide a clearer view as to whether there are sex differences and, if so, in which type of memory they are found.

Likewise, there are other ways in which spatial ability can be measured in the MWM, such as angle of departure from the release position. However, other papers report sex differences using the measures that I have used (Roof \& havens, 1992; Isgor \& Sengelaub, 1998; Cimadevilla et al., 1999), so I think that my measures were sensitive enough to reveal sex differences, had there been any.

In these experiments, I was unable to find sex differences in spatial ability in laboratory rats using either the MWM or the RAM. Manipulations resulting in the subjects learning different aspects of the maze did not have differential effects on the sexes, which suggests that there are no sex differences in spatial learning. However, there were some indications of sex differences in other behaviours (notably exploration behaviours that could be related to stress or food motivation in the RAM, and stress-related behaviours in the MWM) that could potentially cause sex differences in spatial ability.

To conclude, the results of these experiments suggest that stress and motivation may differentially affect male and female performance on spatial ability tasks. I have decided to address the effects that these external factors have on the spatial ability of 
Chapter 2: Do male and female rats learn spatial tasks differently?

male and female rats, rather than continuing to manipulate the tasks until sex differences are found. Experiments intended to address these effects are described in chapters 4 and 5 , respectively. 


\section{Chapter 3: Does oestrous cycle stage affect the performance of female laboratory rats on a reference memory water maze task?}

\subsection{Introduction}

Activational levels of the hormones oestrogen and progesterone are known to affect female spatial ability (section 1.3.2.2). Much of the evidence for these effects has come from studies of the natural fluctuations in female hormones during fertility (menstrual and oestrous) cycles. The majority of studies in humans, rats and rhesus monkeys (Macaca mulatta) have found that poor performance on spatial tasks is correlated with high oestrogen and progesterone cycle phases (e.g. Hampson \& Kimura, 1988; Hampson, 1990; Frye, 1994; Moody, 1997; Phillips \& Silverman, 1997; Warren \& Juraska, 1997; Hausmann et al., 2000; Lacreuse et al., 2001; McCormick \& Teillon, 2001; but see Gordon \& Lee, 1993; Berry et al., 1997; Stackman et al., 1997; Epting \& Overman, 1998; Healy et al., 1999; Postma et al., 1999; Frick \& Berger-Sweeney, 2001; Halpern \& Tan, 2001; Mumenthaler et al., 2001). Similarly, when ovariectomised rats are given hormones to mimic oestrous cycle phases, those given hormones that mimic the levels present during the high progesterone and oestrogen phase (pro-oestrus, figure 1.5) perform worse on a spatial task than those given lower levels of progesterone and oestrogen to mimic di-oestrus (Frye, 1994).

As levels of progesterone and oestrogen co-vary during fertility cycles it is unclear from such studies whether one or the other, or both hormones mediate reduced spatial ability. Studies that have attempted to untangle the effects of these hormones on spatial ability have produced inconclusive results (Chesler \& Juraska, 2000; Hausmann et al., 2000; Sandstrom \& Williams, 2001; discussed in section 1.3.2.2).

The 'fertility and parental care' hypothesis offers an explanation as to why spatial ability should vary across fertility cycles (Sherry \& Hampson, 1997). The 
hypothesis states that spatial ability is lowered in females during reproductive periods such as late pregnancy and lactation in order to reduce predation. Sherry and Hampson (1997) suggest that oestrogen mediates this reduction, and therefore changing levels of the hormone across fertility cycles are correlated with fluctuations in spatial ability due to a by-product of this selected effect of oestrogen.

Oestrous cycle fluctuations in spatial ability could explain the sometimes-found sex differences in spatial ability in laboratory rats (Healy et al., 1999). If the group of females tested included individuals in pro-oestrus, then the mean performance of the females would be lower than that of the males. However, sex differences would not be found if the group of females did not contain any individuals in pro-oestrus.

The studies that have tested spatial ability in female rats at different stages of the natural oestrous cycle have produced somewhat inconsistent results. Two studies found, as expected, that performance on a spatial task was worse during pro-oestrus and best during oestrus when levels of oestrogen are low (Frye, 1994; Warren \& Juraska, 1997). However, two other studies have been unable to find a difference in performance between oestrus and pro-oestrus females (Berry et al., 1997; Stackman et al., 1997), and Healy et al. (1999) found that oestrus females performed worst and pro-oestrus females performed best in the water maze.

These conflicting results may be due to differences in experimental technique between the studies. For example, Stackman et al. (1997) used an appetitively motivated radial arm maze to test spatial ability. Their subjects were on a food restriction protocol, which may have disrupted their oestrous cycles (Tropp \& Marcus, 2001a). The other four studies used water maze spatial tasks that did not involve food restriction. Another difference that may have had an effect was that the two studies that did not find changes in spatial performance across the oestrous cycle did not have a group of male subjects to compare the females to. Instead they made comparisons between females at different oestrous cycle stages (Berry et al., 1997; Stackman et al., 1997). Male rats may be an appropriate control group, as in Frye's (1994) study the only significant difference was between male and pro-oestrus 
females and there were no significant differences between the performances of the pro-oestrus and oestrus female groups.

As well as changes in behaviour during the rat oestrous cycle, there are also neuronal changes. The hippocampal region has been implicated in spatial tasks such as the water maze (e.g. Morris et al., 1982). The number of synapses on certain neurons (CA1 pyramidal cells) in the hippocampus fluctuates during the rat oestrous cycle. The greatest number of synapses is found during pro-oestrus and the least during oestrus (Woolley et al., 1990a). The electrophysiological properties of the hippocampus also vary during the oestrous cycle. Hippocampal neurones display greatest synaptic plasticity (as measured by long-term potentiation) during prooestrus (Warren et al., 1995). These results intuitively suggest that spatial ability should improve during pro-oestrus, as found by Healy et al. (1999). However, the neural data are also consistent with the behavioural data from Frye (1994) and Warren and Juraska's (1997) rat studies, if, rather than increasing learning ability, the rapid addition of flexible synapses actually results in additional noise in the system and so disrupts spatial learning (Warren \& Juraska, 1997).

The rat studies are not alone in finding inconsistent fertility cycle effects. As in Healy et al.'s (1999) study, enhanced spatial ability during high oestrogen phases of the cycle has also been found in single studies of mice and humans (Postma et al., 1999; Frick \& Berger-Sweeney, 2001). There are also a number of non-rat studies that have found no relationship between fertility cycle and spatial ability (Gordon $\&$ Lee, 1993; Epting \& Overman, 1998; Halpern \& Tan, 2001; Mumenthaler et al., 2001). These inconsistencies may be due to difficulties in determining the stage of the fertility cycle that subjects are in, this can be especially difficult as stages associated with high hormone levels may only last a few hours. However, the consensus from the cross-species studies of both natural cycles and hormonal manipulations is that spatial ability is reduced during high oestrogen phases of the cycle.

All of the studies that have examined the effects of oestrous cycle stage on spatial ability in laboratory rats have tested learning and recall of a working memory 
protocol, where memories had to be recalled after no longer than one day (Frye, 1994; Berry et al., 1997; Stackman et al., 1997; Warren \& Juraska, 1997; Healy et al., 1999). I have been unable to find any studies that tested whether oestrous cycle stage affects learning and recall of a reference memory task.

In this study I tested whether oestrous cycle stage affected the recall of a reference memory task. I predicted that the spatial working memory effects would generalise to reference memory, and therefore female rats at the high oestrogen stage of the oestrous cycle (pro-oestrus) would have impaired spatial reference memory recall compared to females at the other stages of the oestrous cycle, and male rats. It is reasonable to make this prediction because working and reference memory can be thought of as two aspects of the same process. Working memories can be consolidated into longer-term reference memories. Evidence for this sequential dependence comes from the observation that agents that inhibit working memory, such as potassium chloride and NMDA receptor antagonists, also prevent the formation of reference memory. Other agents, such as protein synthesis inhibitors, only inhibit the formation of reference memory (Gibbs \& $\mathrm{Ng}, 1977$; Rosenzweig \& Leiman, 1982; Rosenzweig et al., 2002). The evolutionary hypothesis that predicts that oestrogen should reduce spatial ability (the fertility and parental care hypothesis, section 1.2.2), states that the effect will be present during 'reproductive periods'. These are likely to have a timescale of weeks or even months, and therefore it seems likely that oestrogen will affect spatial reference memory as well as spatial working memory.

The reference memory spatial task used was a Morris water maze (MWM). Oestrous cycle stage effects were examined on individual probe trials after reference memory training, rather than during the training itself. This avoided a complicated repeatedmeasures design as the oestrous cycle stage of individual female rats changed from day to day during the reference memory training. This experiment is analysed in with reference to sex differences in spatial ability in section 2.3 , but here it is reanalysed for oestrous cycle effects. 


\subsection{Pilot study}

\subsubsection{Materials and methods}

\section{Subjects and maze training}

A pilot study was carried out during May-June 2001 in order to familiarise myself with the techniques involved. Subjects were six male and six female SpragueDawley rats, previously used for the experiment described in chapter 4 . They were approximately 20 weeks old at the start of the pilot study and were housed in the animal house under the conditions described in section 2.2.2. Food was available ad libitum in their home cages throughout the study. All of the female rats had vaginal smears taken for 14 days prior to, and throughout, the study.

The methods used were similar to those described in section 2.3.2. The subjects had no pretraining in the MWM. A single platform location was used. The order in which the subjects went into the maze and the release position used for each session were fully randomised. The subjects were given three trials per day in the MWM for five days. Each trial consisted of the subject being released into the water from the designated release position and being allowed to swim until the platform was found. If the subject did not find the platform within $2 \mathrm{~min}$ the experimenter guided it to it. The subject was left on the platform for $30 \mathrm{sec}$ before being removed from the maze, dried, and retumed to its home cage. On the sixth day they were given a probe trial, which consisted of a $60 \mathrm{sec}$ swim in the maze from which the platform had been removed.

\section{Smearing}

Smears were taken by restraining the rat and using a plastic pipette to flush a small quantity of sterile saline (approx $0.25 \mathrm{ml}$ ) in and out of the vagina two or three times. A drop of the saline/cell mixture was placed onto a glass slide. The smears were fixed and stained with Giemsa. In order to do this they were first air-dried, once dry the slides were fixed in methanol for $1 \mathrm{~min}$, and then washed with buffer solution. Excess buffer was allowed to drain off and the slide was stained in Giemsa solution 
for $30 \mathrm{~min}$. The slide was again washed with buffer, and the back of it dried with a tissue.

The slides were examined under a microscope using a x10 objective lens and the proportions of cell types present were recorded. Pipettes and slides were rinsed in clean water and reused, after checking that this did not result in contamination.

Both the naming of stages and the expected cell types at each stage varies somewhat between sources (e.g. Waynforth \& Flecknall, 1992; Shors, 1998) but the general consensus is that there are four stages: oestrus, met-oestrus, di-oestrus and prooestrus (figure 3.1). An oestrus smear consists almost entirely of large, irregularshaped, non-nucleated, cornified epithelial cells. A pro-oestrus smear contains mainly round, nucleated intermediate cells. Met-oestrus and di-oestrus smears are less easy to differentiate. Met-oestrus smears consist of a mixture of leukocytes and intermediate cells, while di-oestrus smears are a mixture of all three cell types with leucocytes predominating. Di-oestrus is the longest stage of the cycle, lasting up to 2.5 days; pro-oestrus lasts approximately half a day, as does oestrus; met-oestrus lasts from half to one day. This results in a total cycle of between four and five days.

\subsubsection{Results and analysis}

None of the previous studies had reported differences in performance between the met-oestrus and di-oestrus females so these were grouped together in order to increase sample size. During the probe trial two females were in pro-oestrus and four were in met or di-oestrus, none were in oestrus. There was a trend for females in pro-oestrus to spend less time in the 'correct' quadrant (i.e. the one in which they had been trained to find the platform) than the met/di-oestrus females and the males (figure 3.2).

Statistical analyses were carried out using Minitab. The general linear model (GLM) 'time = group' (equivalent to a one-way analysis of variance or ANOVA) was applied to the data, the groups were male, pro-oestrus, or met/di-oestrus. The data were not transformed. There was no significant effect of group on the time spent in the correct quadrant $\left(\mathrm{F}_{2,9}=2.77, \mathrm{p}=0.116\right)$. A two-tailed, two-sample $\mathrm{t}$-test of the pro- 
oestrus versus met/di-oestrus groups' times was also carried out. This was also nonsignificant $(\mathrm{DF}=4, \mathrm{~T}=-1.72, \mathrm{p}=0.16$, assuming equal variances).

Due to the small sample size non-parametric tests were also used to test for differences between the stages. The Kruskal-Wallis test was used as an alternative to the one-way analysis of variance. There were no significant differences between the groups ( $\mathrm{DF}=2, \mathrm{H}=3.30, \mathrm{p}=0.192$ adjusted for ties). The Mann-Whitney test was used as the non-parametric equivalent of the two-sample $t$-test, the results from this were also non-significant $(\mathrm{W}=4.0, \mathrm{p}=0.247)$.

Figure 3.1: Typical proportions of cell types in vaginal smears at the four stages of the rat oestrous cycle.

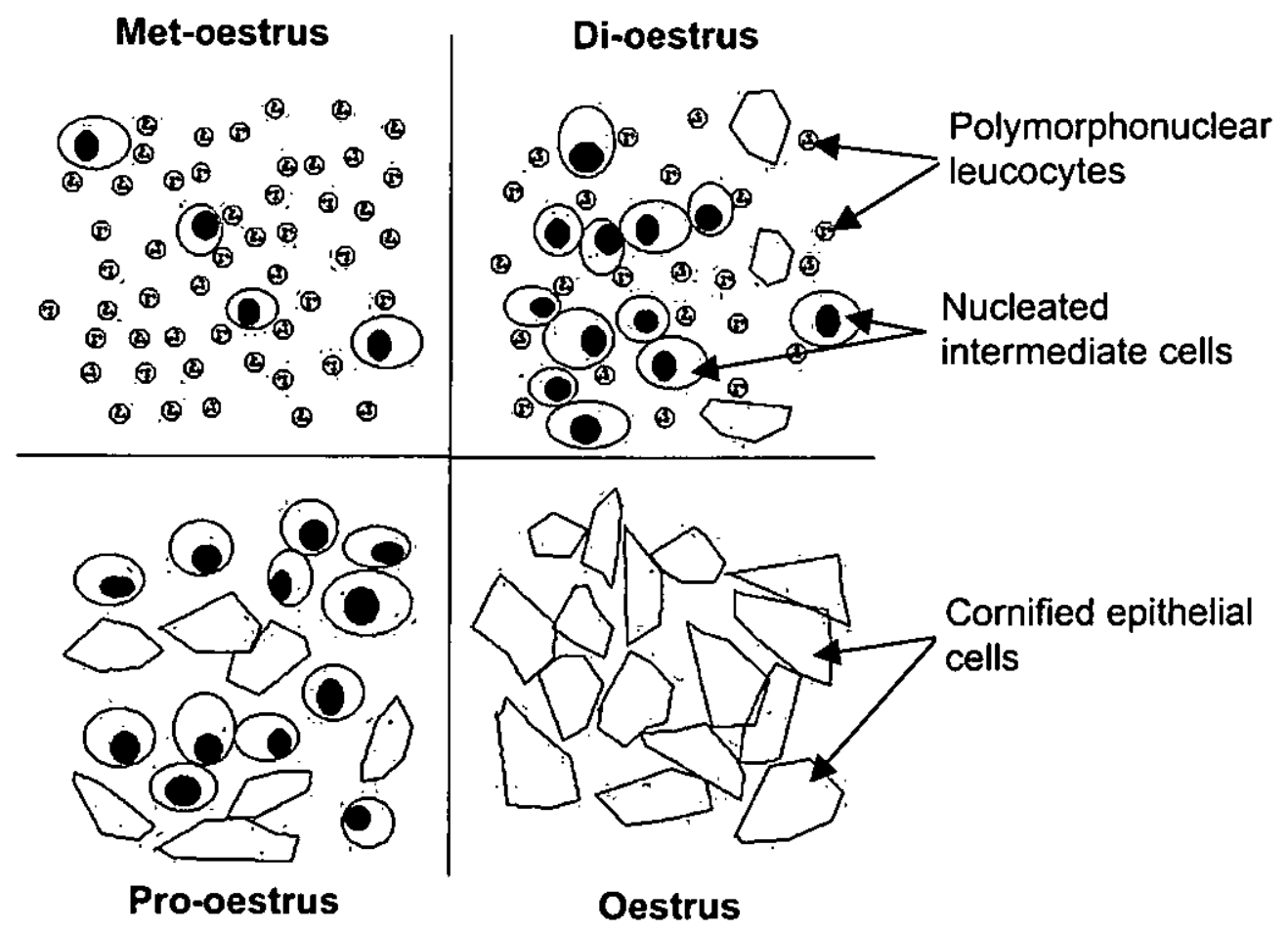


Figure 3.2: Time spent in the 'correct' quadrant during the pilot study probe trial $+/$ standard error of the mean (SEM). Dashed line indicates random expectation of 15sec. Male $n=6$, pro-oestrus $n=2$, met/di-oestrus $n=4$.

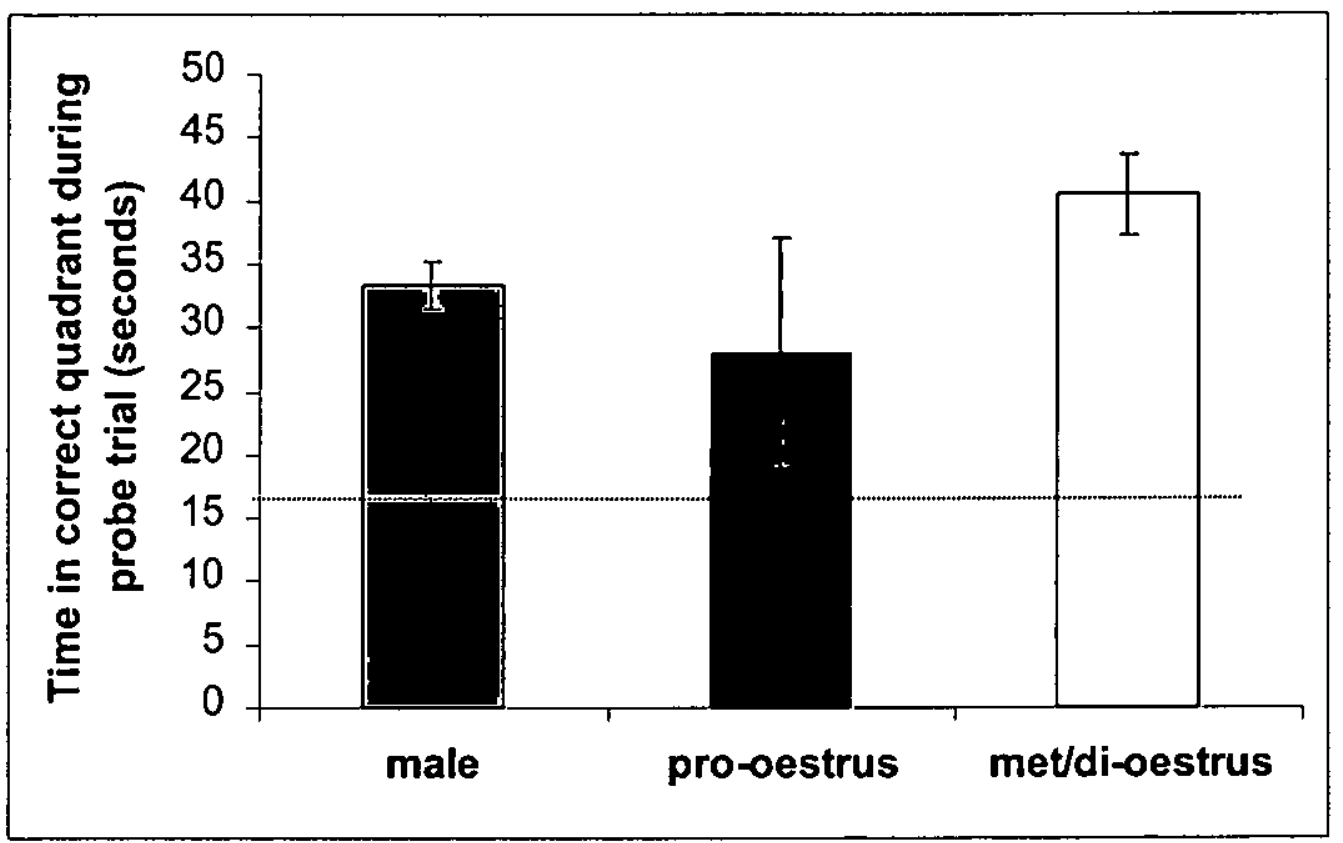

Power analyses were carried out using G*Power (Faul \& Erdfelder, 1992). A post hoc power analysis of the one-way ANOVA had a power of 0.2975 , while the t-test of the pro-oestrus versus met/di-oestrus groups had a power of 0.2175 . Power is defined as $1-B$, where $B$ is the probability of accepting the null hypothesis when it is false (i.e. concluding that there are no differences between the groups when there really are; Buchner et al., 1997). Therefore, power should be as large as possible so as to be likely to get a statistically significant result when there actually is a difference between the groups. The levels of power for the pilot study were very low and therefore it is unlikely that statistically significant differences would be found, even if there were actually differences between the groups.

A priori power analyses were also carried out to estimate how many subjects should be used in the main experiment. These showed that for the one-way ANOVA (time = group) a total of 36 subjects would give a power of $0.8,24$ subjects would reduce the power to 0.6 . For a two-sample t-test between the pro-oestrus and met/di-oestrus 
Chapter 3: Oestrous cycle effects in a reference memory water maze task?

females a total group size of 22 would give a power of $0.8,16$ subjects would result in a power of 0.65 .

\subsubsection{Discussion}

The results of the pilot study suggested that the female rat's oestrous cycle stage may affect recall of the water maze reference memory task in the predicted direction. The power analyses suggested that a reasonable level of power could be obtained, and therefore there would be a good chance of getting a statistically significant difference if there actually is a difference between the groups, by increasing the sample size. Time and space constrained subjects to a maximum of 24 ; therefore it was decided to use eight males and 16 females, which was expected to give a power greater than 0.6 for the planned comparisons.

\subsection{Main experiment}

\subsubsection{Methods}

\section{Subjects and maze training}

For detailed information about the subjects, maze, experimental protocol \& data collection see section 2.3.2. Briefly, the subjects were eight male \& 16 female young adult, experimentally naïve, Sprague-Dawley rats. They were housed in the animal house under the conditions described in section 2.2.2 and had food was available ad libitum in their home cages throughout the study. They were trained twice a day on a MWM hidden platform protocol until they had learnt the platform location; they were then given a $60 \mathrm{sec}$ probe trial without the platform. Training was repeated twice more with different platform locations. Each subject was given a total of five probe trials: the first before any training was given; the second, third and fourth after each of the training stages; and the fifth eight days after the fourth probe trial, during which time the subjects were given no water maze training.

\section{Smearing}


Four days after arriving in the animal house the subjects were handled and marked with coloured ink on their tails to allow identification. The next day smearing began; this was carried out daily for 14 days before water maze training commenced, and throughout the training. Throughout the experiment smearing took place at approximately 1000 , before the first trial in the water maze. Males were also removed from their cages and restrained for a similar period of time as the females in order to control for any handling effects.

The method used for smearing was the same as that used in the pilot study, with the exception that the slides were not stained but viewed while still wet, as it was found that the cell types could be clearly differentiated in this way. All female subjects had at least two consecutive four day cycles before water maze training began, and continued to exhibit regular four (or very occasionally five) day cycles throughout the experiment.

\subsubsection{Results and analysis}

The group sizes were as follows: probe one; oestrus $n=8$, pro-oestrus $n=4$, met/dioestrus $n=4$. Probe two; oestrus $n=5$, pro-oestrus $n=2$, met/di-oestrus $n=9$. Probe three; oestrus $n=2$, pro-oestrus $n=2$, met/di-oestrus $n=12$. Probe four; oestrus $n=3$, pro-oestrus $n=7$, met/di-oestrus $n=6$. Probe five oestrus $n=3$, pro-oestrus $n=6$, met/di-oestrus $n=7$.

The GLM 'response = group' was used to test for time in the quadrant in which they had been trained to find the platform (correct quadrant) on probes 1-5; thigmotaxis on probes 1-5; swim speed on probes 1 and 4; and relative time spent in the half of the water maze furthest from the release position on probe 1 . There were no significant differences on any of the tests (table 3.1). Due to the small and unequal group sizes equivalent Kruskal-Wallis non-parametric tests were also carried out. None of these results were significant either (table 3.1).

There were several non-significant trends: pro-oestrus females tended to spend more time near the release position during the first probe trial (figure 3.3); on the three probe trials after training stages (probes 2,3 and 4 ) the pro-oestrus females tended to 
Chapter 3: Oestrous cycle effects in a reference memory water maze task?

perform worse than the males (figure 3.4); and the oestrus females tended to show less thigmotaxic behaviour than females at other stages of the cycle (figure 3.5). There were no apparent trends in swim speed (figure 3.6).

Two-sample, two-tailed t-tests, and their non-parametric equivalents, were carried out between the male and pro-oestrus female groups on the three probe trials immediately after the training stages (probes 2,3, and 4) as this was where an effect was predicted (figure 3.4). None of the tests were significant (table 3.2).

Figure 3.3: Time spent in the near and far halves of the water maze relative to the release position, during the first probe trial +/- SEM. See text for number of subjects per group. Dashed line indicates random expectation of $30 \mathrm{sec}$.

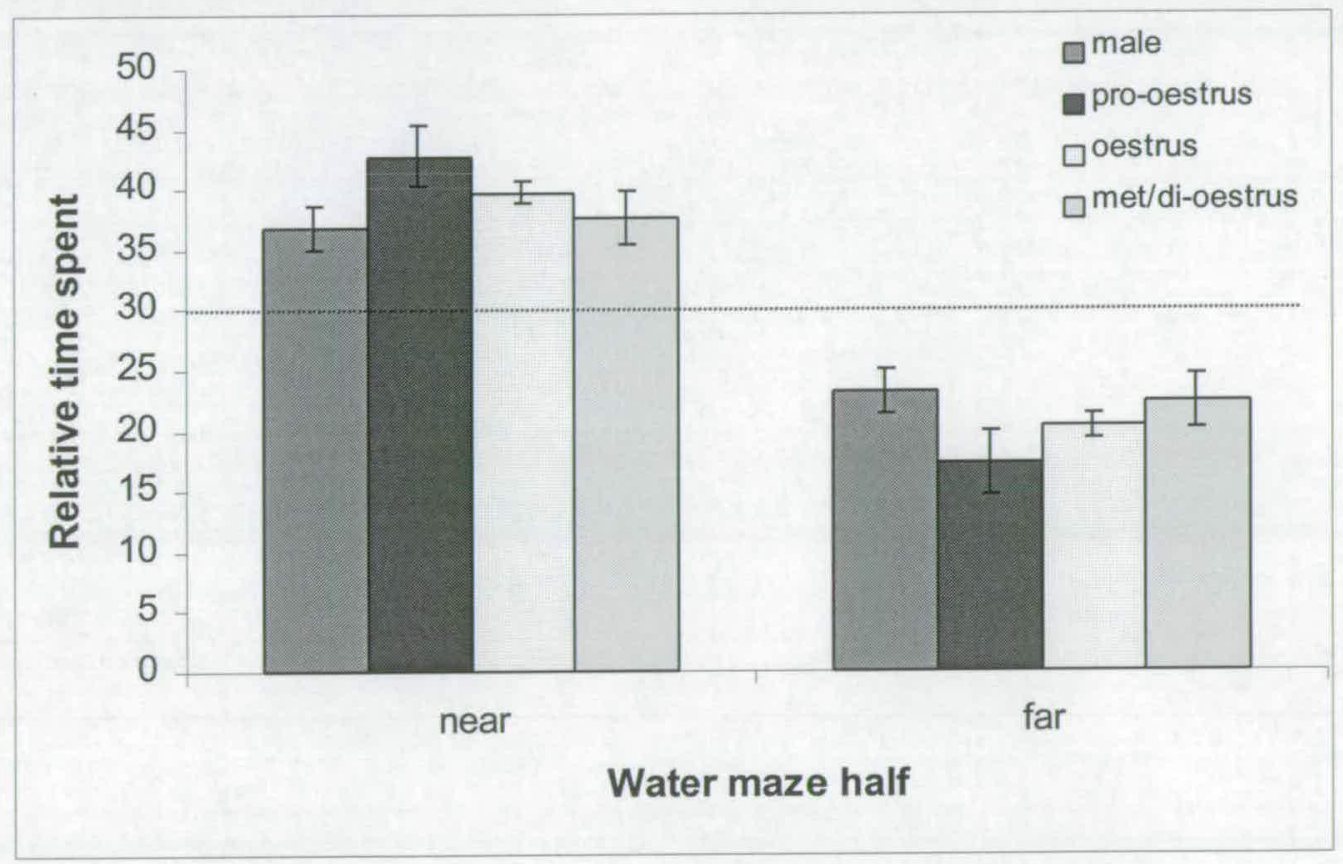


Table 3.1: Results from the parametric and non-parametric tests for an effect of group (male, pro-oestrus female, oestrus female, met/di-oestrus female) on parameters in a MWM reference memory spatial task. See text for numbers of subjects in each group. GLM degrees of freedom $(D F)=3,20$. All Kruskal-Wallis tests are adjusted for ties, $D F=3$. Time in correct quadrant on probe 1 was squareroot transformed for the GLM analysis. None of the test statistics had p-values of less than 0.1 .

\begin{tabular}{|c|c|c|c|}
\hline Probe & Response & $\begin{array}{l}\text { GLM response=group } \\
\text { (F-ratio) }\end{array}$ & $\begin{array}{l}\text { Kruskal-Wallis test } \\
\text { (H statistic) }\end{array}$ \\
\hline 1 & $\begin{array}{l}\text { Time in correct } \\
\text { quadrant }\end{array}$ & 0.50 & 1.19 \\
\hline 2 & $\begin{array}{l}\text { Time in correct } \\
\text { quadrant }\end{array}$ & 1.25 & 3.88 \\
\hline 3 & $\begin{array}{l}\text { Time in correct } \\
\text { quadrant }\end{array}$ & 0.72 & 2.18 \\
\hline 4 & $\begin{array}{l}\text { Time in correct } \\
\text { quadrant }\end{array}$ & 0.31 & 0.96 \\
\hline 5 & $\begin{array}{l}\text { Time in correct } \\
\text { quadrant }\end{array}$ & 1.17 & 2.97 \\
\hline 1 & Thigmotaxis & 1.43 & 4.80 \\
\hline 2 & Thigmotaxis & 1.00 & 3.14 \\
\hline 3 & Thigmotaxis & 0.39 & 1.69 \\
\hline 4 & Thigmotaxis & 0.35 & 1.56 \\
\hline 5 & Thigmotaxis & 0.42 & 2.04 \\
\hline 1 & Swim speed & 0.21 & 1.13 \\
\hline 4 & Swim speed & 0.20 & 0.65 \\
\hline 1 & $\begin{array}{l}\text { Time spent near } \\
\text { release position }\end{array}$ & 1.92 & 3.34 \\
\hline
\end{tabular}


Figure 3.4: Time spent in the 'correct' quadrant during the five probe trials in the main experiment $+/$ - SEM. Dashed line indicates random expectation of $15 \mathrm{sec}$. See text for number of subjects per group.

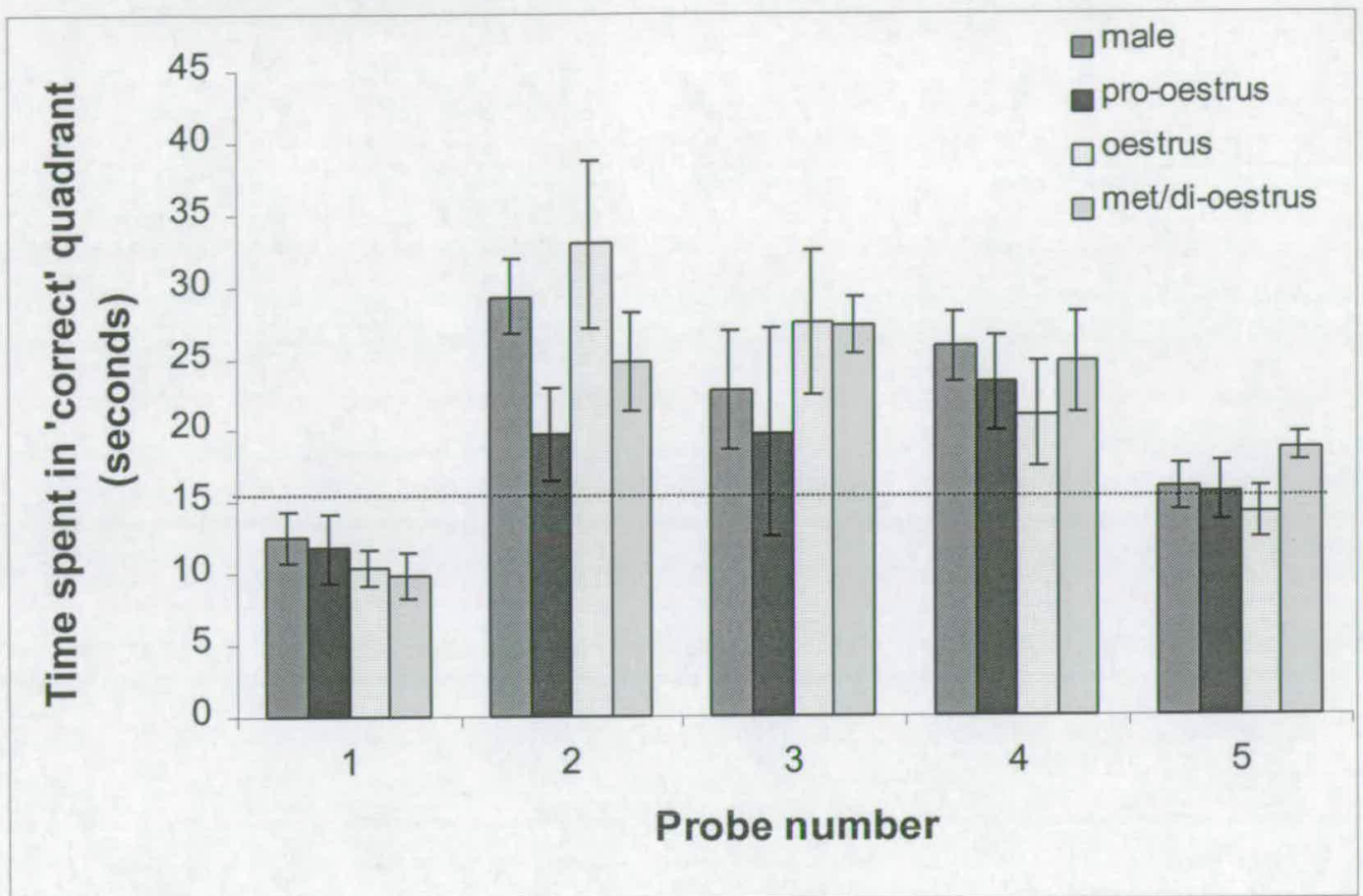

Figure 3.5: Time spent in thigmotaxic behaviour during the five main experiment probe trials $+/$ - SEM. See text for number of subjects per group.

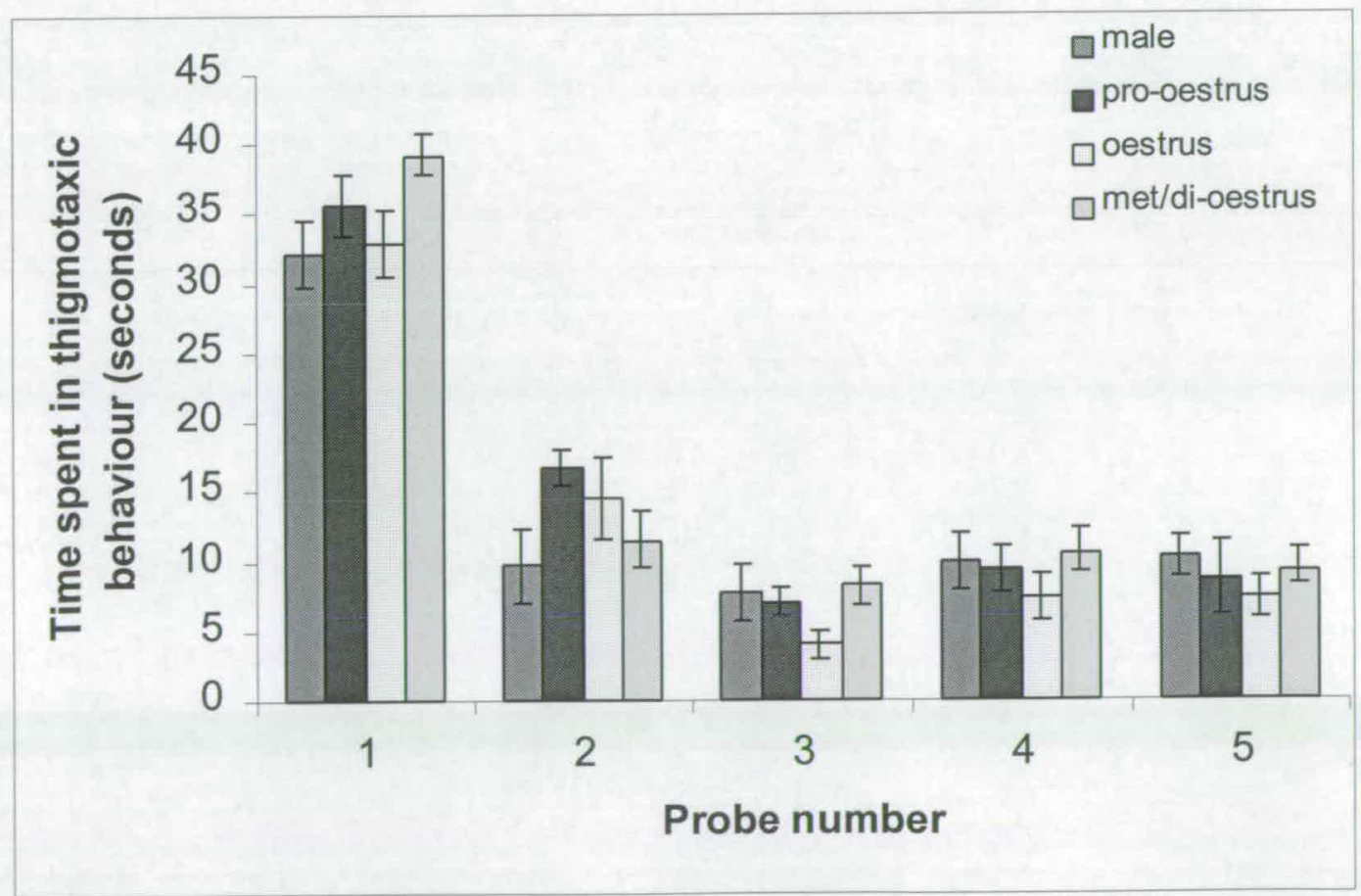


Chapter 3: Oestrous cycle effects in a reference memory water maze task?

Figure 3.6: Swim speed, calculated as distance travelled in 30 seconds in arbitrary units, during the first and fourth probe trials +/- SEM. See text for number of subjects per group.

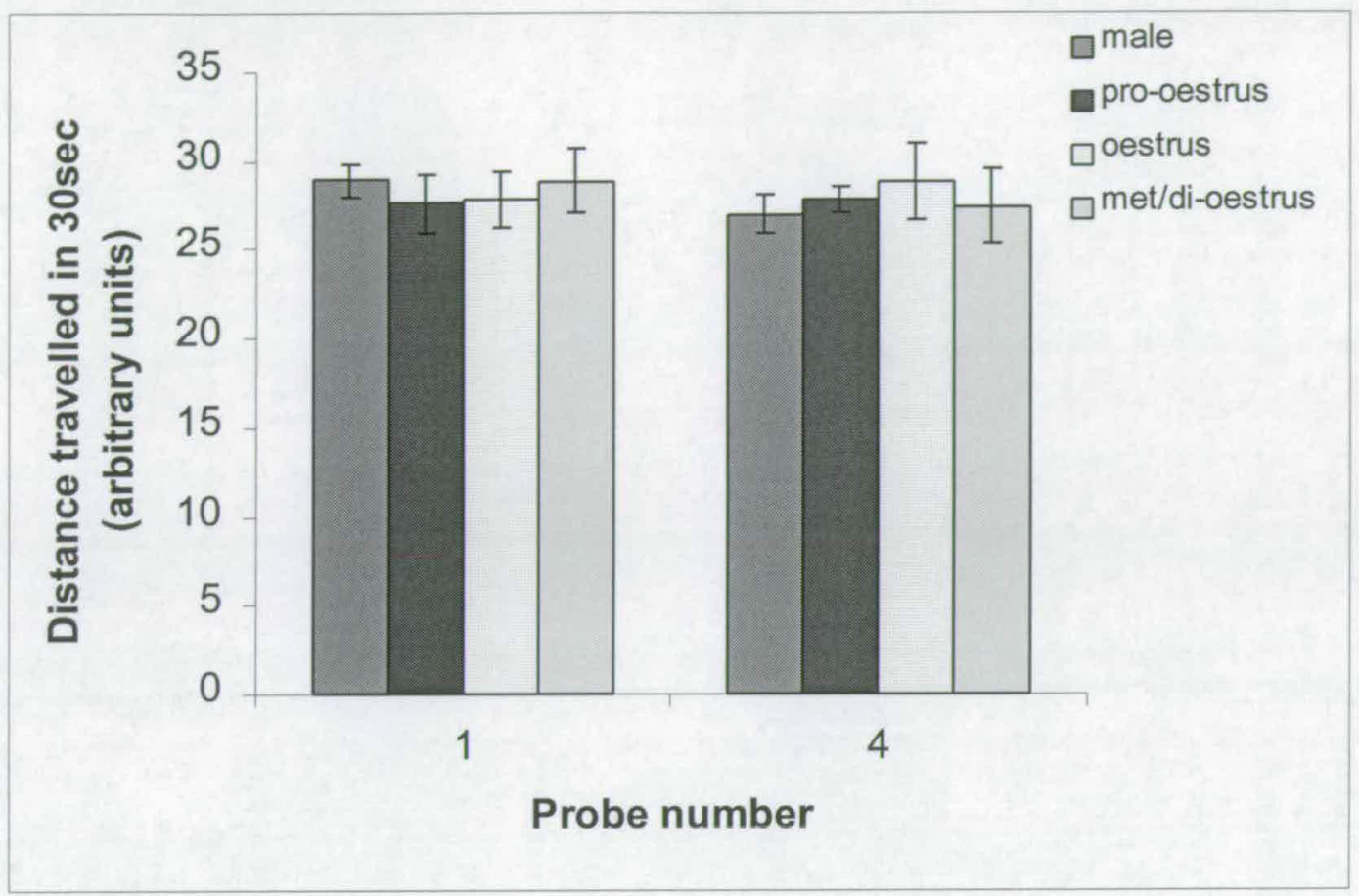

Table 3.2: Results from the parametric and non-parametric tests for a difference between the male and pro-oestrus female groups on time spent in the correct quadrant during probe trial after training in a MWM reference memory spatial task. See text for numbers of subjects in each group. The two-sample t-test was two-way and assumed equal variances. None of the test statistics had p-values of less than 0.1 .

\begin{tabular}{l|l|l|l}
$\begin{array}{l}\text { Probe } \\
\text { trial }\end{array}$ & $\begin{array}{l}\text { Degrees of } \\
\text { freedom }\end{array}$ & $\begin{array}{l}\text { Two-sample t-test (T } \\
\text { statistic) }\end{array}$ & $\begin{array}{l}\text { Mann-Whitney test } \mathbf{W} \\
\text { statistic) }\end{array}$ \\
\hline 2 & 8 & 0.32 & 50.5 \\
3 & 8 & 0.13 & 47.0 \\
4 & 13 & 0.55 & 68.5
\end{tabular}

Post hoc power calculations were carried out on the three probe trials immediately after the training stages (probes 2, 3, and 4) for both one-way ANOVAs to test for differences between the groups, and for two-sample, two-tailed t-tests between the 
Chapter 3: Oestrous cycle effects in a reference memory water maze task?

male and pro-oestrus female groups (figure 3.4). The results showed that power was low for the second probe trial $(0.3425$ for the one-way ANOVA, 0.5735 for the twosample t-test), and extremely low for the third and second probe trials (below 0.2 for all tests). These figures were lower than the power analysis on the pilot study had suggested that they would be. A priori power calculations suggested that in order to increase the power to reasonable levels a much larger sample size would be necessary (table 3.3).

Table 3.3: Results from a priori power analyses using the data obtained in the main experiment. The ANOVA model used was 'time spent in correct quadrant = group', the t-test was two-sample, two-tailed between the male and pro-oestrus female groups.

\begin{tabular}{l|l|l|l} 
Probe & Statistical test & Power & $\begin{array}{l}\text { Total number of } \\
\text { samples needed }\end{array}$ \\
\hline 2 & ANOVA & 0.6 & 44 \\
2 & ANOVA & 0.8 & 64 \\
2 & t-test & 0.6 & 10 \\
2 & t-test & 0.8 & 12 \\
3 & ANOVA & 0.6 & 84 \\
3 & ANOVA & 0.8 & 124 \\
3 & t-test & 0.6 & 204 \\
3 & t-test & 0.8 & 350 \\
4 & ANOVA & 0.6 & 176 \\
4 & ANOVA & 0.8 & 264 \\
4 & t-test & 0.6 & 144 \\
4 & t-test & 0.8 & 244
\end{tabular}




\subsection{Discussion}

This experiment found no significant differences in the recall of a reference memory water maze task between female rats at different oestrous cycle stages and males. However, the study suffered from low statistical power and therefore I am unable to reliably conclude that such differences do not exist. One of the problems with the study was that the females' oestrous cycle was not controlled and this resulted in small and unequal group sizes for some of the probe trials. Power could be increased by using more subjects and/or by having equal numbers of subjects in each group. Therefore, perhaps the easiest way to overcome this problem would be to use a larger sample size of female rats. Alternatively, ovariectomised females given hormone replacement therapy to mimic the oestrous cycle phases could be tested. This would ensure that there were equal numbers of subjects in each phase. However, such subjects would be exposed to constant levels of hormones for several days before and during the experiment rather than experiencing the natural hormonal fluctuations of the oestrous cycle and therefore the data obtained from them may not reflect the effects of the natural oestrous cycle. It may be possible to overcome this problem by giving the ovariectomised rats hormone injections, instead of implants (e.g. Chesler \& Juraska, 2000).

Another problem with my experiment was that no allowance was made for that fact that the females may have had different amounts of time in each cycle stage during training. This could have an effect if learning a reference memory task is impaired at certain cycle phases. This could be partially controlled for in future experiments by training the subjects for multiples of four days (which is the average length of the rat oestrous cycle) before the probe trials. Under this protocol each subject would have equal numbers of training days at each cycle stage, albeit at different parts of the training stage.

The data suggest that it might be productive to continue with this line of study, for example on probe two (the first memory probe) the results are in expected direction, with the pro-oestrus females spending the least time in the correct quadrant of any of the groups (figure 3.3). 
The data also show a trend for pro-oestrus females to display more anxiety-related behaviour than the other females and the males. On the first and second probes the pro-oestrus females show more thigmotaxic behaviour than the males (figure 3.5), the pro-oestrus females also spend more time near the release position on probe one than the other groups (figure 3.4). These behaviours could be interpreted as being related to increased anxiety (e.g. Perrot-Sinal et al., 1996).

Increased anxiety could in turn result in poor apparent spatial ability as tested in the water maze, and therefore may be a possible explanation as to why pro-oestrus female rats are often found to have poor spatial ability. Other studies have found that reducing stress and anxiety improves spatial performance in female rodents (Galea et al., 1994c; Perrot-Sinal et al., 1996; Kavaliers et al., 1996).

Data from stress hormones supports the trends apparent in my data. Serum levels of glucocorticoid hormones are positively correlated with stress in rats (Armario et al., 1986). Pro-oestrus females have higher resting levels of corticosterone and higher levels of corticosterone in response to stress than di-oestrus females (Critchlow et al., 1963; Viau \& Meaney, 1991).

However, the trends apparent in my data and from the hormonal studies are in conflict with tests of anxiety across the oestrous cycle. Using tests of anxiety such as the elevated plus maze pro-oestrus females show less anxious behaviour than females at other stages of the oestrous cycle (Frye et al., 2000; Marcondes et al., 2001). However these tests may not be appropriate measures of anxiety in female rats as they were designed and tested for male rats. A factor analysis study by Fernandes et al. (1999) suggests that females' behaviour on the elevated plus maze is more influenced by exploration factors, unlike the males' behaviour, which as expected, is driven by anxiety.

To conclude, I think it would be useful to repeat this study with a larger sample size, and also to train the rats on the water maze for multiples of four days in order to try to control for any oestrous cycle effects on learning. This would enable a more powerful test for effects of oestrous cycle on recall of a reference memory task, and 
Chapter 3: Oestrous cycle effects in a reference memory water maze task? also allow tests to see if anxiety-related behaviour in the water maze changes across the oestrous cycle, as the hormonal evidence suggests that it might. 


\section{Chapter 4: The effects of acute restraint stress on the performance of male and female laboratory rats in the radial arm maze}

\subsection{Introduction}

One possible explanation for the observed sex differences in spatial ability in laboratory rats is that they are caused by differences in male and female rats' response to stress (section 1.4). Stress can be defined as a state that occurs

"when an animal encounters adverse physical or emotional conditions which cause a
disturbance of its normal physiological and mental equilibrium" (Manser, 1992)

Stress can be experimentally induced using aversive experiences such as electric shocks or physical restraint.

Stress can be classified as either acute or chronic: acute stress is short-term and usually an adaptive response to the situation in which the animal finds itself; chronic stress, on the other hand, is long-term and is often considered to be maladaptive as the sustained stress response has adverse effects (Bowman et al., 2001). There is evidence that chronic stress can affect spatial ability, as male rats that were confined in a narrow tube (restraint stress) for 6hr a day over 21 days had impaired performance in both Y and radial arm mazes (Luine et al., 1994; Conrad et al., 1996).

Acute stress can also affect spatial ability. Stillman et al. (1998) restrained male rats in either warm or cold water for $15 \mathrm{~min}$ before testing them in a radial arm maze (RAM). They found an additive effect of stress such that the rats that were restrained in cold water performed worse than the subjects that were restrained in warm water, which in turn were impaired compared to an unstressed control group. Similarly, tail-shock stress impaired learning of a working memory RAM task in male rats (Shors \& Dryer, 1992). 
Stress hormones such as the fast-acting catecholamines (e.g. adrenaline) and sloweracting glucocorticoids (e.g. corticosterone) are known to affect attention and memory consolidation processes (reviewed in McEwen \& Sapolsky, 1995; Mendl, 1999). Excessively high or prolonged elevations of glucocorticoids, similar to those present during chronic stress, result in more glucocorticoid binding to low-affinity type II receptors in the hippocampus, which inhibits long-term potentiation. Such levels also cause damage to hippocampal neurons, such as atrophy of the apical dendrites of pyramidal cells in the CA3 region of the hippocampus (figure 1.3), and probably mediate the deleterious effects of chronic stress on spatial ability (e.g. Woolley et al., 1990b; Watanabe et al., 1992).

The effects of stress hormones can also explain the negative effects of acute stress. When a subject is given an injection of adrenaline shortly after an event the formation of memories of that event are enhanced. However, increasing the dose decreases this facilitating effect. Catecholamines do not cross the blood-brain barrier, so it is thought that their effects on peripheral glucose mobilisation and cerebral perfusion rate (which result in more glucose reaching the brain), may mediate their effects on memory. Like adrenaline, glucose also enhances memory at intermediate but not high levels, probably by stimulating neurotransmitter and receptor synthesis (Mendl, 1999).

There are reports of sex differences in the effects of both acute and chronic stressors on cognition, although the direction of the effect varies between studies. For example, although $6 \mathrm{hr}$ of restraint stress a day for 21 days impairs RAM performance in males, it appears to improve performance in female rats (Bowman et al., 2001). Acute stress in the form of electric shocks to the tail of a restrained rat facilitates the acquisition of classical conditioning in males, but impairs acquisition in females (Wood \& Shors, 1998). Conversely, the same stressor impairs instrumental learning in males, but has little effect on female rats (reviewed in Shors, 1998).

To summarise, stress has been shown to affect spatial ability, and there are sex differences in the effects of stress on performance of various cognitive tasks. There 
are three lines of evidence that suggest that sex differences in stress response could cause sex differences in spatial ability (reviewed in section 1.4). Briefly: first, sex differences in spatial ability in rats are often only apparent during the initial stages of the task, when the novelty of the task would make it more stressful (Manser, 1992); second, sex differences appear to be less likely to occur when extensive pretraining is given, and therefore novelty and stress are reduced (Bucci et al., 1995; Perrot-Sinal et al., 1996; Warren \& Juraska, 1997; Healy et al., 1999; Markowska, 1999); third, studies in meadow voles and deer mice have shown that when endogenous opiate activity is inhibited sex differences in spatial ability are reduced (Galea et al., 1994c; Kavaliers et al., 1996). Increased opiate activity is associated with stress. Therefore, it is possible that female rodents are more stressed than males by some maze tasks and that this impairs their apparent spatial ability, especially during the early stages of testing when the task is novel.

If the amount of stress associated with the spatial test varies between laboratories, then stress could also potentially explain why some studies find sex differences in spatial ability in rats while others do not. The amount of handling, previous experience with similar tasks, or familiarity with the test room could all influence how stressful a task is, and therefore possibly affect whether sex differences are found or not.

The uneaten reward errors that resulted in apparent sex differences in spatial ability during my previous RAM experiment (section 2.2) may also be related to stress. These errors were most frequent at the beginning of the experiment before decreasing rapidly to low frequency, a pattern that that would be expected of a stressrelated behaviour induced by novelty. It is possible that if the spatial task induces a higher stress response in females this could reduce their motivation to eat, as stress has been shown to suppress appetite (Pare, 1965).

In this experiment the effects of sex and stress on performance in a partially rewarded RAM were studied. $15 \mathrm{~min}$ in a restraint tube was used to induce stress immediately prior to each trial in the maze. A relatively mild acute stressor was used because I thought that it would more accurately replicate the stress induced in the 
rats by the novel environment provided by the maze. Restraint stress was chosen because it has previously been shown to result in greatly elevated corticosterone levels in rats (up to 20 times higher than in unstressed controls; Armario et al., 1986; Kant et al., 1986). Corticosterone is the main glucocorticoid hormone released in response to stress in rats (Manser, 1992).

Previous work carried out in my lab had shown that this level of restraint stress is enough to alter behaviour in the black-white box (Beveridge, 2001). The blackwhite box test involves a box with two compartments, one painted white and the other black. An archway allows access from one compartment to the other. The box is placed in a dark room and the white compartment is illuminated while the black compartment remains dark (figure 5.2). Rats placed in the box are assumed to experience conflicting motivations: exploring the whole box versus avoiding the bright compartment. The amount of time that an individual spends in the dark side is a measure of how anxious they are (e.g. Bilkei-Gorzó et al., 1998; Henniger et al., 2000). Restrained rats showed more anxious behaviour and in this context anxiety can be equated with stress.

Four groups of rats were used in the experiment: restrained males, control males, restrained females and control females. Although this RAM task had not previously resulted in sex differences in spatial ability (section 2.2), the experimental design allowed a direct comparison within each sex. I hypothesised that females would be more stressed by the novel maze environment than males, and that this would reduce their spatial ability, either directly, or indirectly by making them less likely to eat the rewards. If the hypothesis was correct, I predicted that the male restrained group would display reduced spatial ability compared to the control males, but I did not expect there to be such a large difference between the female groups as the females would already be maximally stressed by the task and the additional stress would not make much difference.

\subsection{Materials and methods}

\section{Subjects and maze}


The experiment was carried out during February-April 2001. 12 male and 12 female experimentally naïve Sprague-Dawley rats, weighing between 175-200g, were supplied by B\&K Universal Ltd. They were housed in same sex-pairs in the same animal house and conditions described in section 2.2.2. The subjects were allowed to settle into the animal house for seven days before being weighed and put on the food restriction regime described in section 2.2.2. Prior to pretraining the rats were introduced to the food cups used in the maze by leaving one in each cage for two days, and to the food rewards (Sainsburys Honey Hoops) by feeding each subject two rewards after weighing for five days before pretraining began. The RAM and the room in which it was housed are described in section 2.2.2. One of each pair of cage-mates was randomly assigned to the restraint group.

Each subject was given one trial in the maze per day, seven days a week. The order in which the subjects went in the maze on each day was pseudorandomised, so at least two but not more than four subjects from each of the four groups appeared in each half of the order (i.e. the first 12 subjects to go in the maze, and the second 12 subjects). This was to control for the fact that the endocrine response to stress in rats is known to vary with time of day, it being largest at the beginning of the light period (Kant et al., 1986). The trials started between 0900 and 1000 and finished at approximately 1430 . The basic RAM procedure is described in section 2.2.2.

\section{Restraint tube}

The restraint tube was locally made based on details from Waynforth and Flecknell (1992). It consisted of a 300 $\mathrm{mm}$ long, $65 \mathrm{~mm}$ diameter piece of plastic tubing (figure 4.1). Five slots were cut into the tubing; the first was $185 \mathrm{~mm}$ from the end, the others were at $25 \mathrm{~mm}$ intervals further in. This allowed a metal plate with holes to be inserted to shut off one end of the tube; the slot used depended on the size of the subject. After the rat had been inserted headfirst into the tube the other end was blocked using a rubber cork that had a notch cut in it to allow the rats' tail to hang outside. The entire apparatus was supported with a clamp and stand. 
Figure 4.1: The restraint tube.

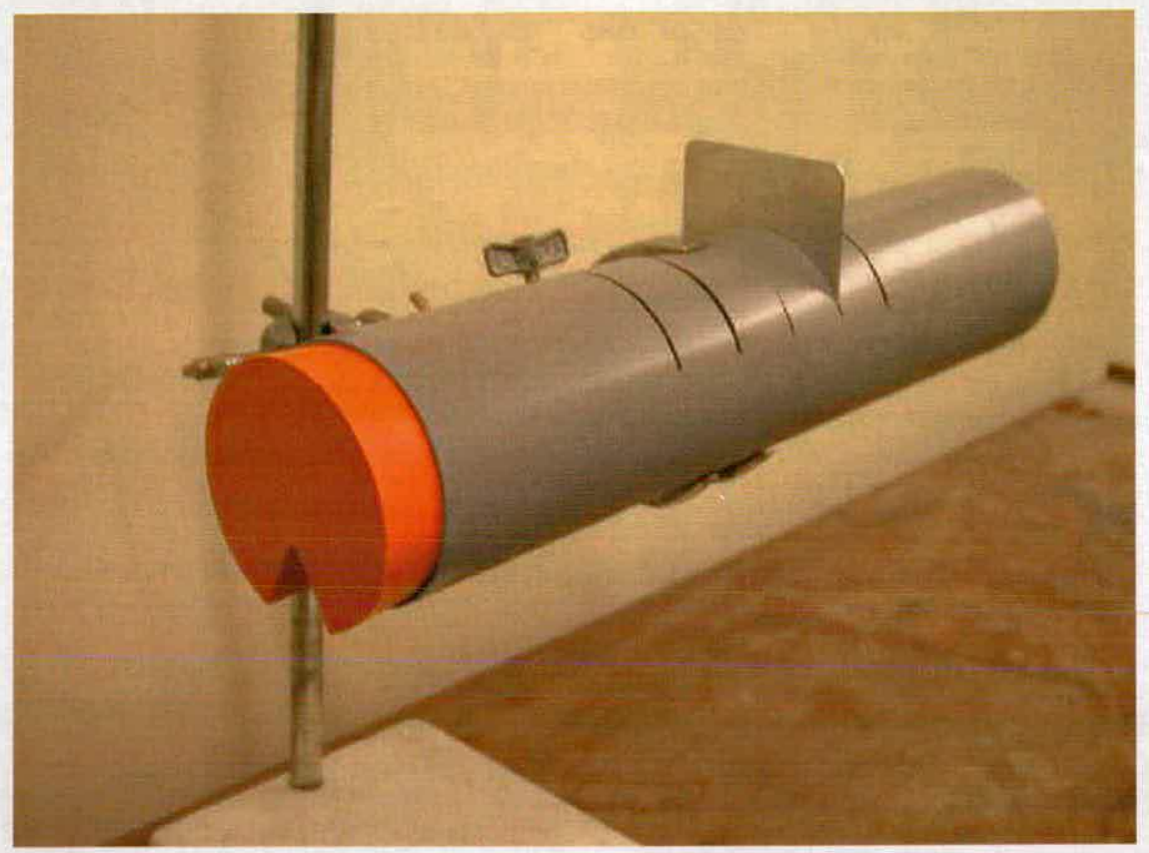

\section{Pretraining}

Pretraining in the radial arm maze began after 12 days, when the rats had stabilised at their target weights. None of the rats were restrained during pretraining. During pretraining all eight cups in the maze contained a reward, resulting in a working memory task designed to familiarise the rats with the procedure and the maze itself. After the release chamber was raised the subject was allowed to explore the maze for $10 \mathrm{~min}$, or until it had eaten all the rewards. The number of rewards eaten by each subject was recorded. One trial was given per day, after 10 days of pretraining all of the rats except one female were eating all the rewards within $10 \mathrm{~min}$. This rat was given extra pretraining sessions on a day when the rest of the rats did not go in the maze. It took six further trials until it ate all eight rewards. The main experiment was started on the next day.

\section{Experiment}

This consisted of a reference memory task where four out of eight RAM arms contained food rewards. Each rat was assigned an individual pattern of four 
rewarded arms. These were pseudorandomly generated and were different for each rat. Patterns with more than two adjacent rewarded arms, and patterns where every other arm was rewarded were excluded in order to discourage the rats from developing strategies of visiting every arm or every adjacent arm. The rats were placed in the maze and allowed to explore until they had eaten all the rewards or until $20 \mathrm{~min}$ had elapsed. They were each given 30 trials. The rats assigned to the restraint group were put in the restraint tube for $15 \mathrm{~min}$ immediately before being placed in the maze. On two occasions different rats from the female restraint group had an adverse reaction to the restraint tube and remained motionless when placed in the maze. In these cases the trial was adjourned while the subject was removed from the maze and handled for a few minutes. After handling the subject was replaced in the maze and the trial proceeded as normal.

\subsection{Results and analysis}

\section{Repeated-measures analysis}

Repeated-measures analyses of variance (ANOVAs) were carried out on the number of reference memory errors made, number of working memory errors, and average time per arm (calculated by dividing the total time taken to complete the maze by the number of arms entered). The data were blocked into blocks of five trials. The following general linear model (GLM) was applied to the data, using Minitab:

Response $=\operatorname{sex}+$ restraint + sex*restraint + rat $($ sex restraint $)+$ block + sex*block + restraint*block + sex $*$ restraint*block

There were two between-subjects effects (sex and restraint) and one within-subject effect (block). As none of the block interactions were significant for any of the response variables they were removed from the model, and all the results reported below are taken from the minimal model. The sex by restraint interaction could not be removed from the model because it was nested in 'rat'. F-ratios for the withinsubject effects were calculated using the mean square (MS) for the 'rat(sex restraint)' 
term as the denominator. The data were checked to see if they met GLM assumptions, and transformations were applied as appropriate.

The block term was significant for both working and reference memory errors (table 4.1). This was due to performance improving over time as the subjects learnt the task (figure 4.2.a\&b). Neither sex nor restraint had a significant effect on either measure of spatial ability (table 4.1).

For time per arm the sex by restraint interaction was approaching significance (table 4.1). Tukey simultaneous tests showed that the only significant difference between the pairs of groups was between the male restraint and female restraint groups $(t=2.863, p=0.0438)$. The restrained females tended to take longer per arm, and the restrained males tended to take less time per arm, than control rats of either sex (figure 4.2.c). The main effect of block was also significant for time per arm (table 4.1), the mean time spent in each arm appeared to decrease as the experiment progressed (figure 4.2.c).

Table 4.1: F-ratios for the repeated-measures ANOVAs including uneaten reward errors. Degrees of freedom for sex, restraint, and sex ${ }^{\star}$ restraint $=1,20$; for block $=$ $5,115 .{ }^{*}=p<0.1,{ }^{* *}=p<0.05,{ }^{* * *}=p<0.01$. Working memory errors were inverse transformed, 'time per arm' was square-root transformed.

\begin{tabular}{l|llll}
\multirow{2}{*}{ Response } & \multicolumn{3}{l}{ Effect } \\
\cline { 2 - 5 } & sex & restraint & sex*restraint & block \\
\hline Reference memory errors & 0.29 & 0.20 & 0.73 & $5.30^{\star \star \star}$ \\
Working memory errors & 0.48 & 2.08 & 1.28 & $10.21^{\star \star \star}$ \\
Time per arm & $5.22^{\star \star}$ & 0.18 & $3.16^{\star}$ & $4.75^{\star \star \star}$
\end{tabular}


Figure 4.2: Performance of the four groups of rats in the radial maze with four arms rewarded out of eight $+/$ - standard error of the mean (SEM). One block is the mean of five trials. $\mathrm{N}=6$ for each group.

a) Reference memory errors (entries into arms that never contain food rewards).

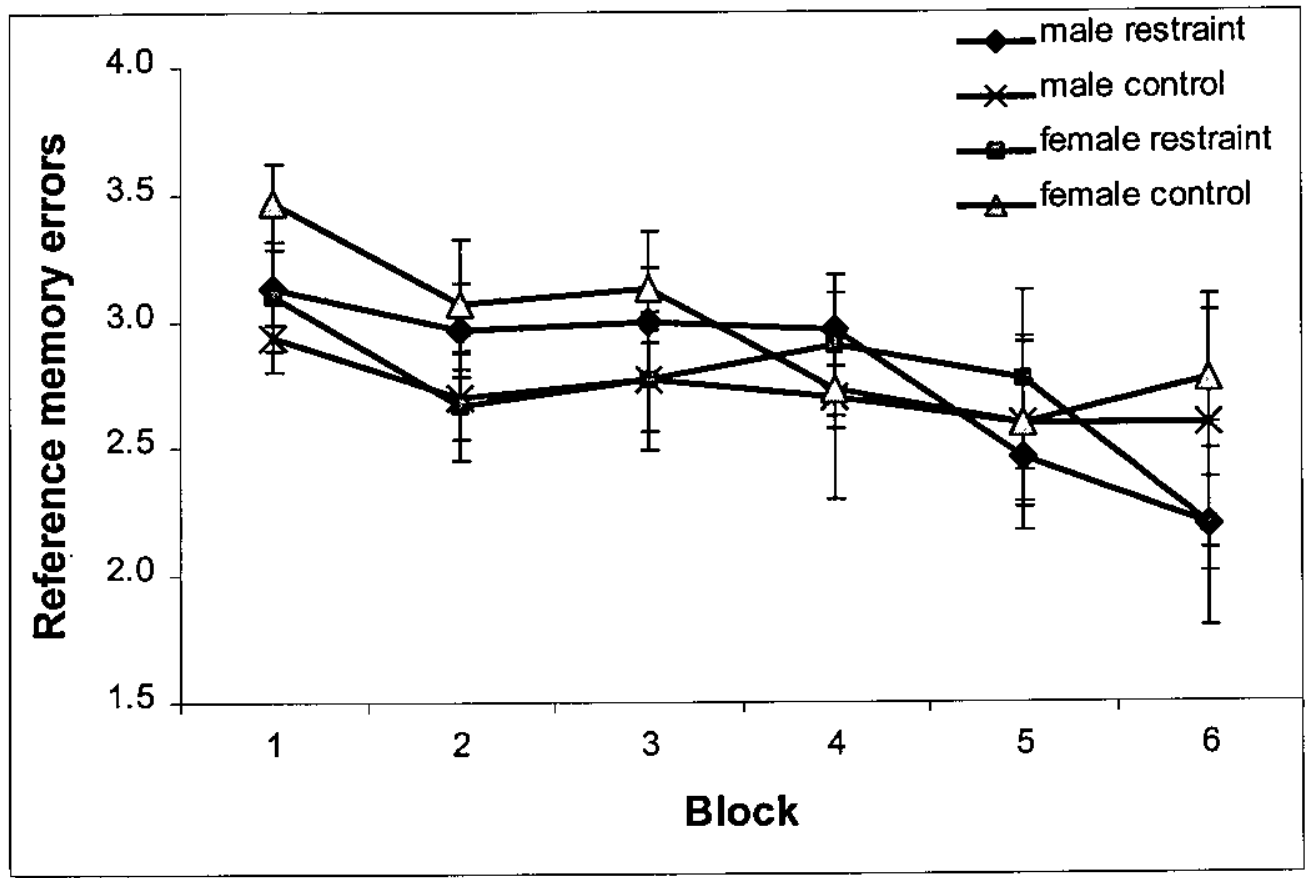

b) Working memory errors (entries into rewarded arms previously visited on that trial that did not contain a reward).

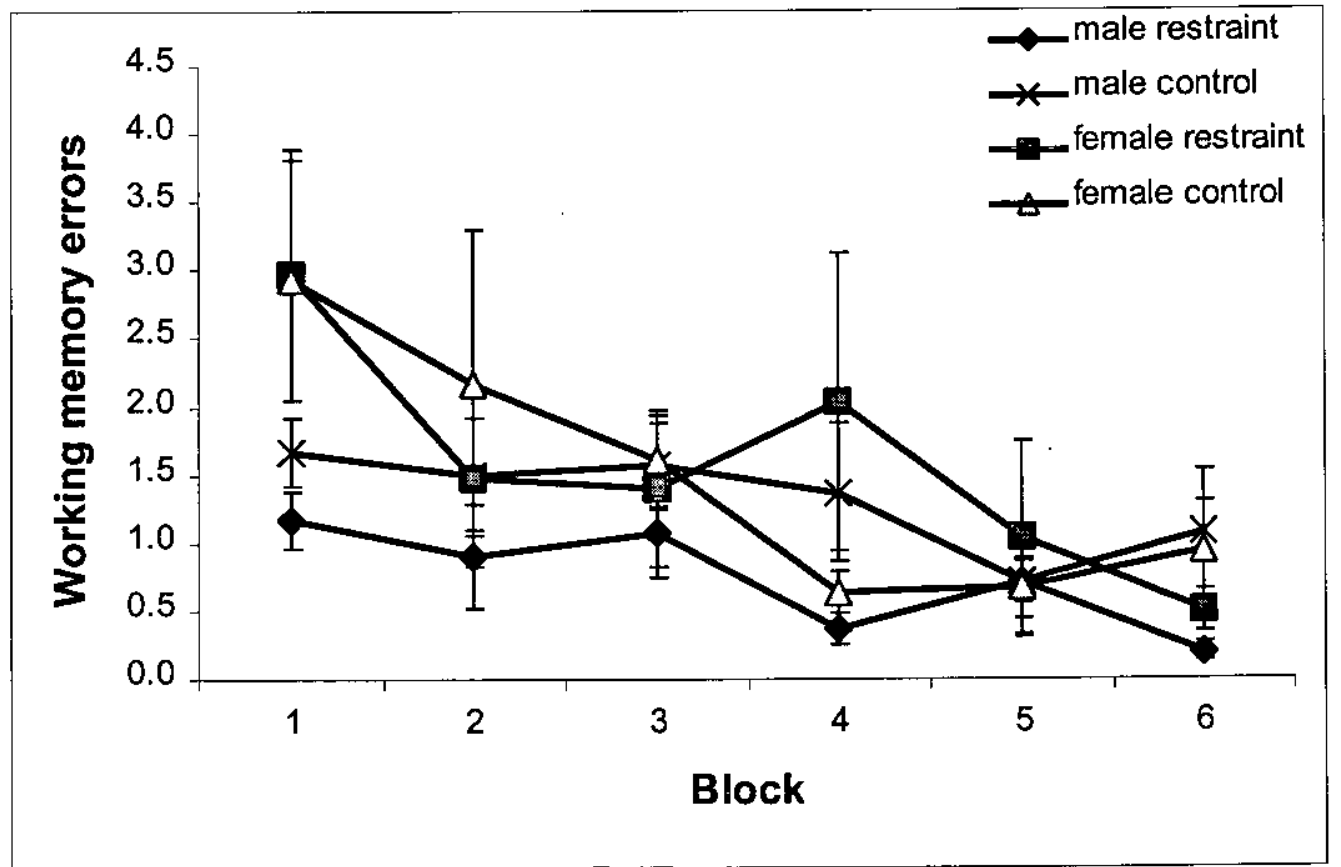


c) Time per arm (total time divided by number of arms entered).

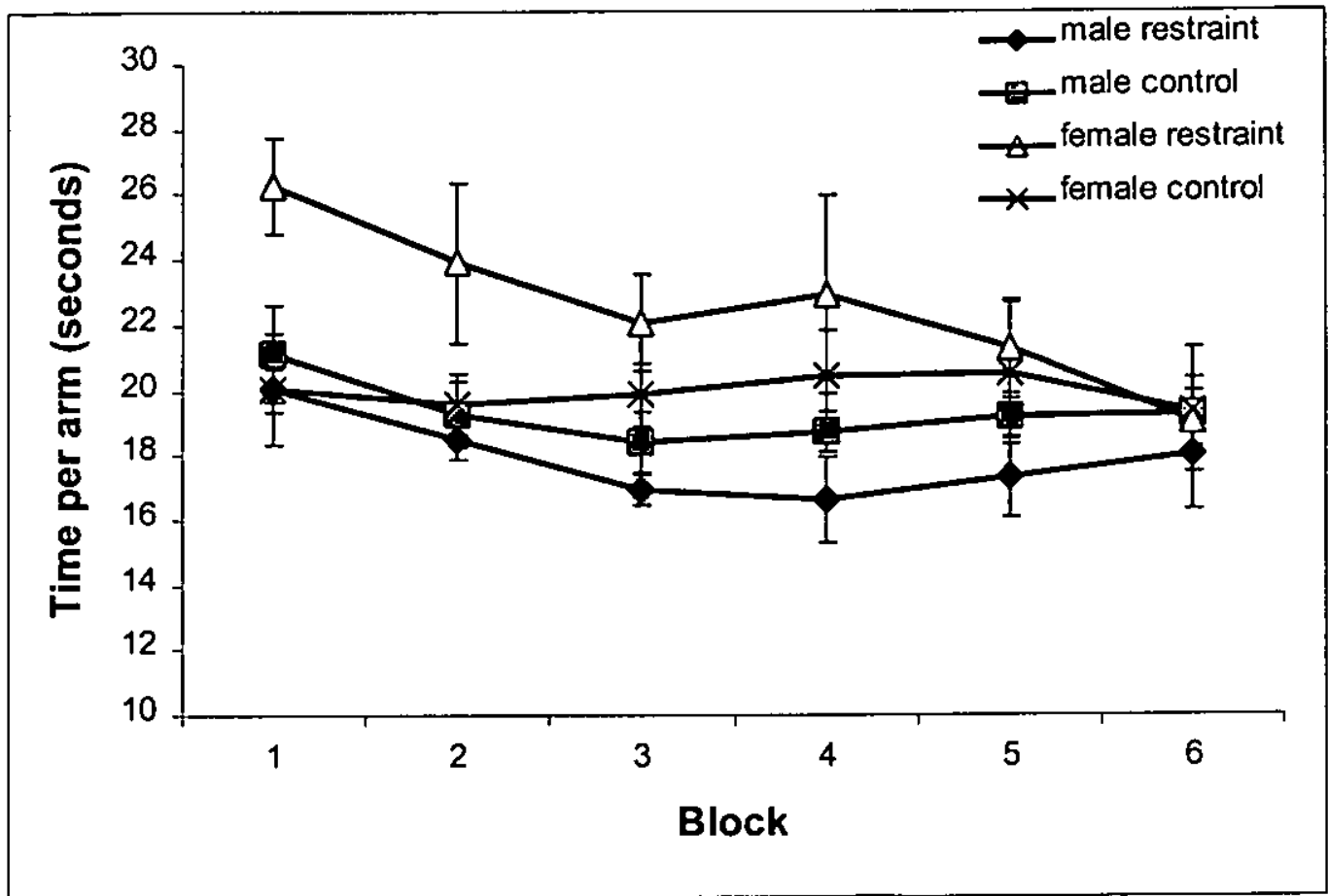

\section{Analysis of first blocks}

Unlike in the RAM experiment in chapter 2 there was no consistent trend for sex differences in the spatial ability measures. Any sex differences were expected to be most apparent at the beginning of the experiment so statistical tests were carried out on the first block of data for both of the spatial ability measures using the following GLM:

Response $=\operatorname{sex}+$ restraint + sex ${ }^{*}$ restraint

None of the interaction terms were significant therefore all of the reported results are taken from the minimal model. The effect of restraint was not significant for either of the spatial ability measures (table 4.2). The effect of sex was not significant for reference memory errors, but was significant for working memory errors (table 4.2). The females made more working memory errors than the males during the first block (figure 4.2.b). 
Table 4.2: F-ratios for 2-way ANOVAs on the first block only. When the interaction term was not significant (ns) it was not included in the analysis. Degrees of freedom for sex and restraint when the interaction was not included $=1,21$. Degrees of freedom for all effects when the interaction was included $=1,20 .{ }^{*}=p<0.1,{ }^{\star *}=p<0.05$, $\star \star \star x=p<0.01$. None of the response variables were transformed.

\begin{tabular}{l|lll|lll}
\multirow{2}{*}{ Response } & \multicolumn{4}{|l|}{ With uneaten reward errors } & \multicolumn{3}{l}{$\begin{array}{l}\text { Without uneaten reward } \\
\text { errors }\end{array}$} \\
\cline { 2 - 7 } & sex & restraint & sex*restraint & sex & restraint & sex*restraint \\
\hline $\begin{array}{l}\text { Reference } \\
\text { memory } \\
\text { errors }\end{array}$ & 2.06 & 0.23 & ns & 0.27 & 1.32 & $6.81^{* \star}$ \\
$\begin{array}{l}\text { Working } \\
\text { memory } \\
\text { errors }\end{array}$ & $5.72^{* *}$ & 0.13 & ns & 1.87 & $3.03^{*}$ & $\mathrm{~ns}$ \\
\end{tabular}

\section{Uneaten reward errors}

As in chapter 2 an uneaten reward error was counted if a subject went into a rewarded arm but failed to eat the reward. The uneaten reward errors data were nonnormal and did not fulfil the parametric test assumptions so they were tested using the Mann-Whitney non-parametric test. Separate Mann-Whitney tests for the effects of sex and restraint were performed on the mean number of uneaten reward errors made by each rat during the 30 trials. There was no effect of restraint on the number of uneaten reward errors made ( $W=148.5, p=0.9526$, adjusted for ties), but there was a significant effect of $\operatorname{sex}(W=78.0, p<0.0001$, adjusted for ties $)$, with females making more uneaten reward errors than males (figure 4.3). 
Figure 4.3: Uneaten reward errors made by the four groups of rats in the radial arm maze $+/-$ SEM. One block is the mean of five trials. $N=6$ for each group.

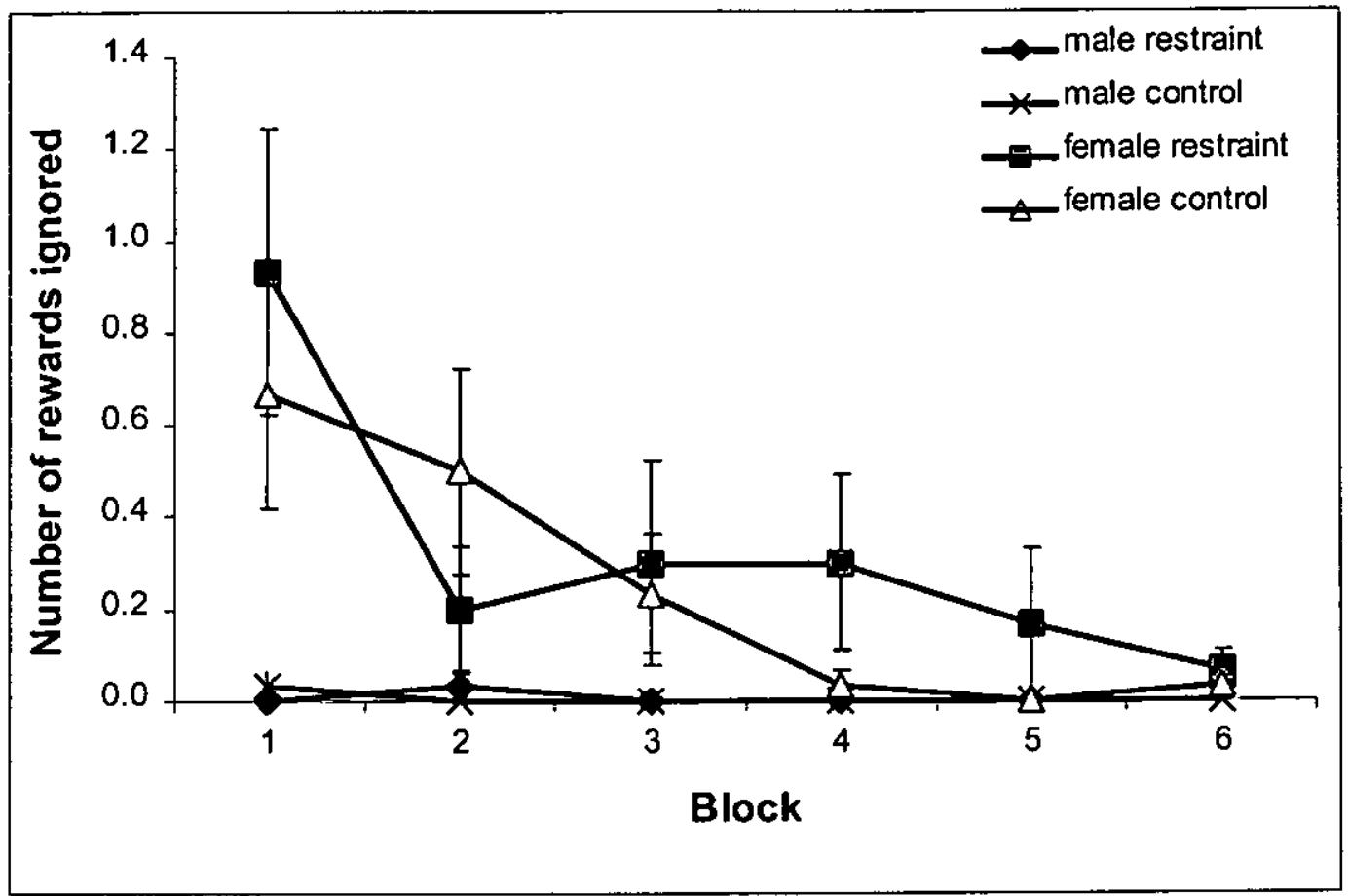

\section{Repeated-measures analysis excluding uneaten reward errors}

As in section 2.2.3, the uneaten reward errors were removed from the raw data and the two spatial ability measures were reanalysed, the repeated-measures ANOVA GLM model with sex and restraint as between-subjects effects and block as the within-subject effect was used. None of the interaction terms involving block were significant and so they were left out of the final analysis.

The results were similar to those from the first analysis (compare figures $4.2 \& 4.4$, also tables $4.1 \& 4.3$ ), with the exception that the effect of restraint became significant for working memory errors (table 4.2). This was due to restrained subjects making fewer errors than control animals (figure 4.4.b). 
Table 4.3: F-ratios for the repeated-measures ANOVAs excluding uneaten reward errors. Degrees of freedom for sex, restraint, and sex* ${ }^{*}$ estraint $=1,20$; for block = $5,115 .{ }^{\star}=p<0.1,{ }^{\star \star}=p<0.05,{ }^{\star \star \star}=p<0.01$. Working memory errors were inversed transformed.

\begin{tabular}{l|llll}
\multirow{2}{*}{ Response } & \multicolumn{3}{l}{ Effect } & \\
\cline { 2 - 5 } & sex & restraint & sex*restraint & block \\
\hline Reference memory errors & 0.17 & 0.29 & 0.79 & $2.93^{\star \star}$ \\
Working memory errors & 0.93 & $5.86^{\star \star}$ & 0.51 & $5.76^{\star \star \star}$
\end{tabular}

\section{Analysis of first blocks excluding uneaten reward errors}

The first blocks were again individually analysed for effects of sex and restraint. None of the data were transformed. The results from this analysis differed from those from the analysis including uneaten reward errors in several ways. The sex by restraint interaction term became significant for reference memory errors (table 4.2). However, Tukey simultaneous tests showed that the only difference between the groups that was nearing significance was between the female restraint group and the female control group $(t=-2.657, p=0.0666)$. The female restraint group tended to made fewer reference memory errors than the female control group (figure 4.4.a). The main effect of sex became non-significant for working memory errors, but the main effect of restraint was approaching significance (table 4.2). This appeared to be due to restrained animals tending to make fewer working memory errors than control (figure 4.4.b) 
Figure 4.4: Spatial performance excluding uneaten reward errors in the eight arm radial maze for the four groups of rats +/- SEM. One block is the mean of five trials. $\mathrm{N}=6$ for each group.

a) Reference memory errors (entries into arms that never contain food rewards).

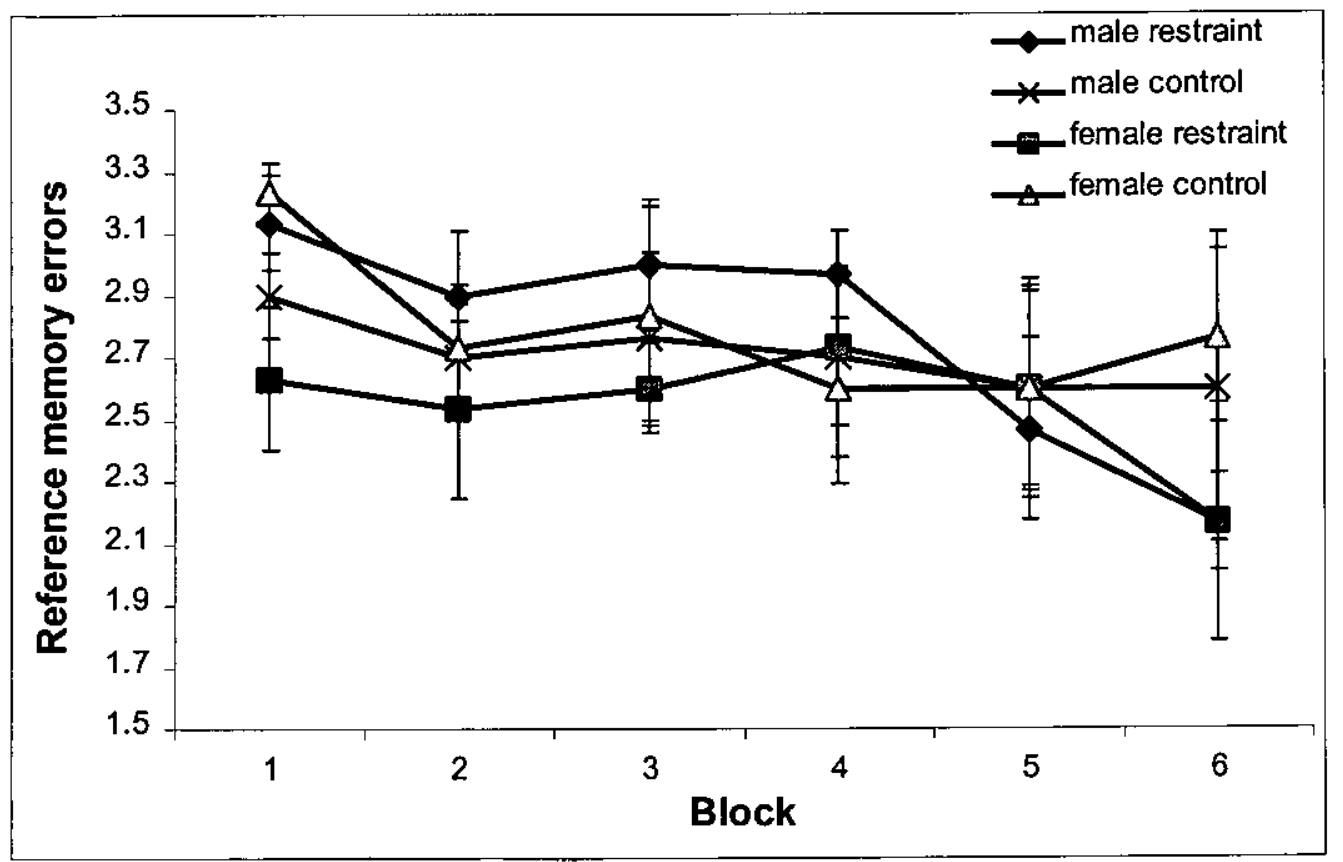

b) Working memory errors (entries into arms previously visited on that trial).

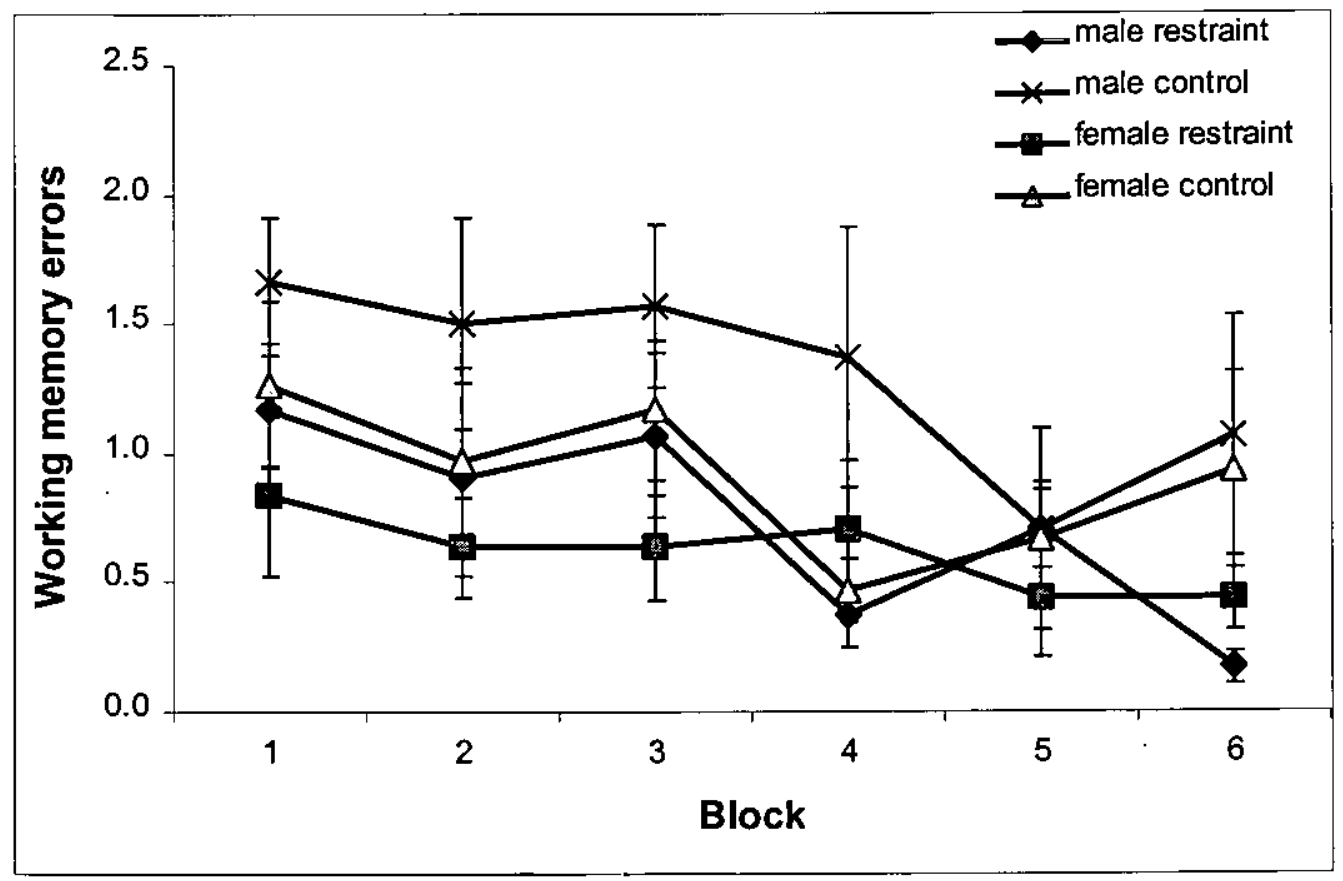




\section{Regressions}

As in the previous RAM experiment (section 2.2.3), the mean number of uneaten reward errors made by each subject during the experiment were regressed individually against sex and restraint, plus one of the following covariates:

a) 'Pretraining': the number of trials that it took the subject to reach the criterion of eating all eight rewards within $10 \mathrm{~min}$ during pretraining.

b) 'Time per arm': the mean time per arm over all 30 trials.

c) 'Weight gain': the weight gained by the subjects during the entire period of food restriction.

Uneaten reward errors were square-root transformed for all analyses. An outlying data-point (a subject from the female restraint group, which made more than twice as many uneaten reward errors as any other subject) was removed from the analysis. Removing this subject did not affect the significance of the effects of stress or restraint on the number of uneaten reward errors made.

Pretraining and time per arm did not have a significant effect on the number of uneaten reward errors made, allowing for sex and restraint (pretraining $\mathrm{F}_{1,19}=1.09$, $\mathrm{p}=0.309$; time per arm $\mathrm{F}_{1,19}=0.01, \mathrm{p}=0.909$ ). Weight gain had an effect approaching significance $\left(\mathrm{F}_{1,19}=3.13, \mathrm{p}=0.093\right)$. Within each group there was a trend for rats that gained more weight to make more uneaten reward errors, but the effect was not very large (figure 4.5).

\section{Effects of restraint on weight gain}

In order to test whether the amount of weight that the subjects gained during the experiment (from the start of food restriction until the last week of RAM training) was affected by the restraint regime the following GLM was used:

Weight gain $=$ sex + restraint + sex $*$ restraint 
The interaction term was not significant and so was left out of the final model. The effect of sex was significant $\left(F_{1,21}=136.01, p<0.001\right)$, males gained more weight than females (figure 4.6). This was to be expected as adult male Sprague-Dawley rats can weigh up to half as much again as similarly-aged females. The effect of restraint was also significant $\left(F_{1,21}=11.77, p=0.003\right)$, the restrained rats gained less weight than those that were not restrained (figure 4.6).

Figure 4.5: Regression plot of mean number of uneaten reward errors made against weight gain during the experiment.

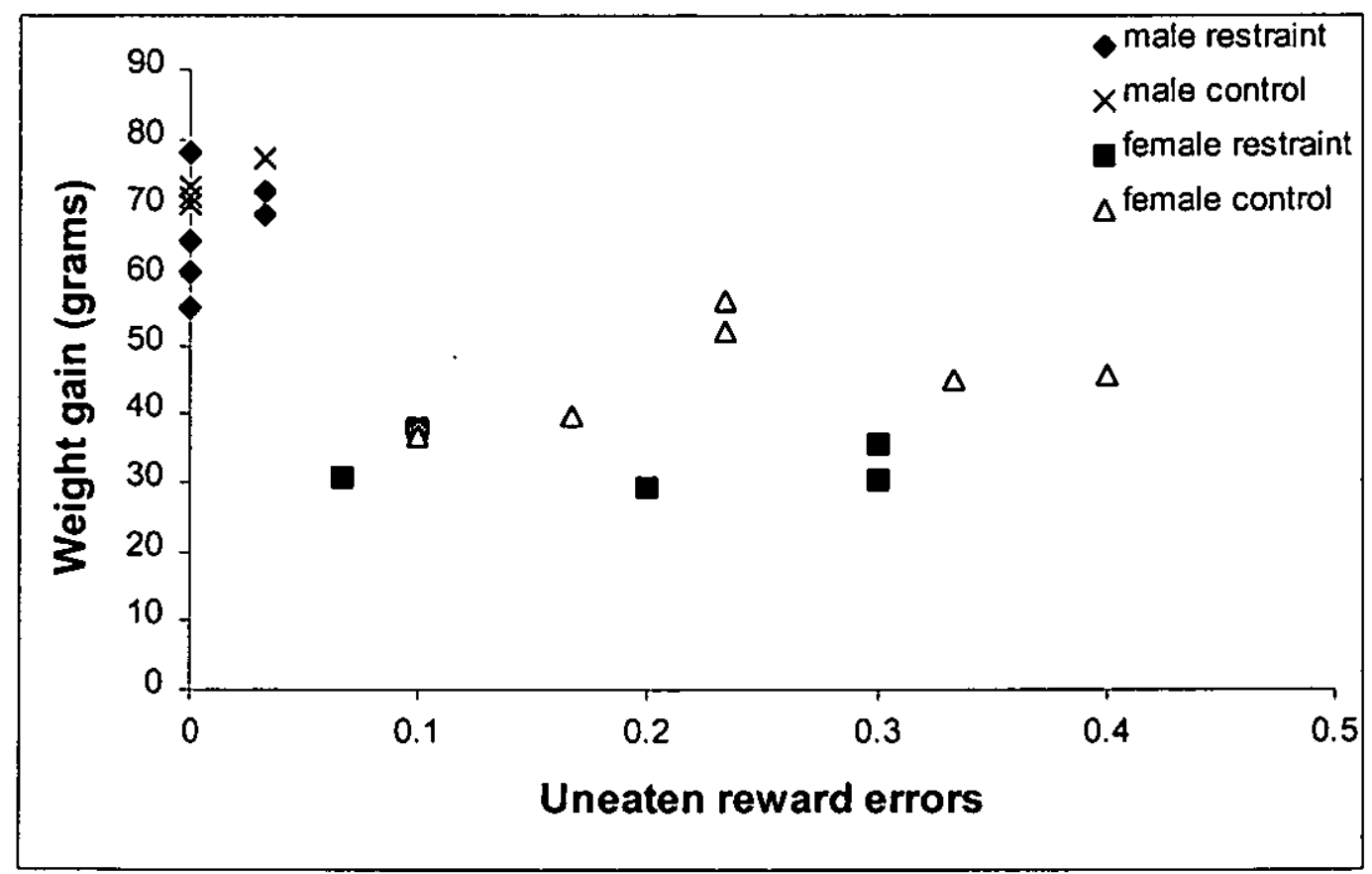


Figure 4.6: Weight gained by the restrained and unrestrained male and female rats during the experiment. $N=6$ for each group, $+/-$ SEM.

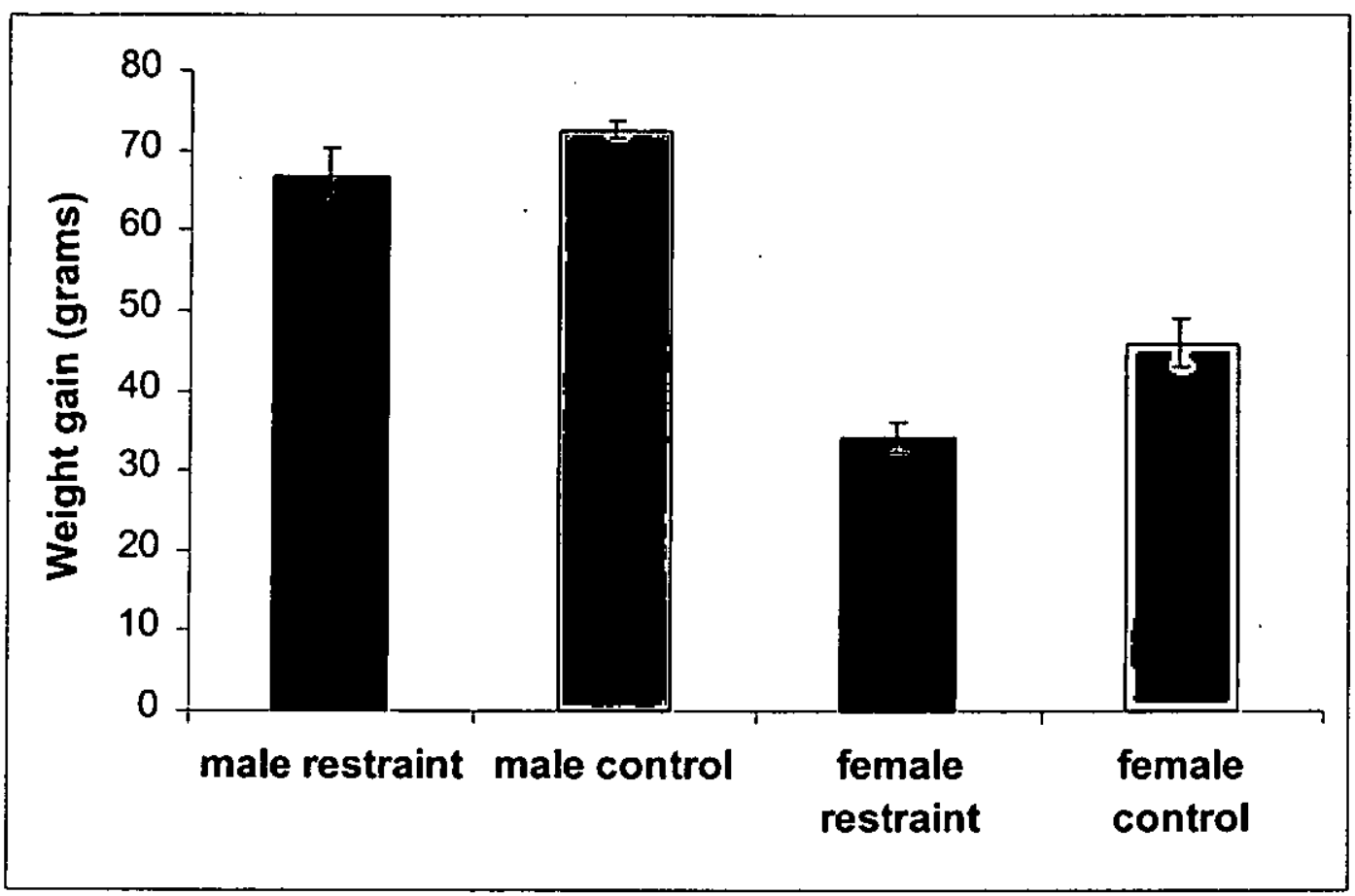

\section{$4.4 \cdot$ Discussion}

Female rats were more likely than male rats to enter a rewarded arm but fail to eat the reward, this replicated the findings of my previous RAM experiment (section 2.2.3). Restraint stress did not affect the subjects' tendency to make uneaten reward errors. This suggests that uneaten reward errors are unlikely to be related to stress, but does not rule out the possibility that they are a result of sex differences in motivation or exploration.

As in the earlier RAM experiment, the sex differences in uneaten reward errors appeared to account for sex differences in spatial ability, as sex differences in spatial ability were no longer apparent when the uneaten reward errors were removed from the data. However, unlike in the previous experiment, sex differences in spatial ability were apparent only during the first block, and not throughout the experiment as a whole. The frequency of uneaten reward errors decreased rapidly as the 
experiment progressed (figures $4.2 \& 2.4$ ); therefore sex differences only during the first block may be explained by the extra pretraining given in this experiment (a total of $100 \mathrm{~min}$ versus $85 \mathrm{~min}$ in the earlier experiment). The extra pretraining may have meant that fewer uneaten reward errors were made at the start, and that they decreased to negligible levels more quickly, in this experiment than in the earlier one. The extra time spent in pretraining during this experiment may also explain why trials to criterion during pretraining did not affect the tendency to make uneaten reward errors, unlike in the previous radial maze experiment.

Although there were no overall sex differences in spatial ability, there were sex differences in activity within the restrained rats. Female restrained rats took significantly longer per arm entry than restrained males. This finding replicates the results of a study by Faraday (2002) who restrained male and female SpragueDawley rats for $20 \mathrm{~min}$ before testing them in an open-field apparatus to measure activity. Faraday found that the stressed females showed reduced activity, but the males were unaffected, compared to control groups of unstressed rats. In my study the effect appeared to be reduced over time, but in Faraday's study activity after stress was only measured for 19 days, and the effect did not change during this time.

When the uneaten reward errors were removed, restrained animals of both sexes made fewer working memory errors than controls. A similar effect was seen in reference memory errors, but only within the females on the first block. These effects are in the opposite direction to my predictions. Although surprising, this facilitating effect of stress is not unprecedented. The 'Yerkes-Dodson law' is used in psychological literature to describe the relationship between stress or arousal and cognitive performance (Lupien \& McEwen, 1997; Mendl, 1999). The law states that the relationship has an inverse U-shape, with maximum performance at intermediate levels of stress. This law could explain these results, if the control rats were so unstressed by the procedure that their performance was below optimum, the additional stress experienced by the restrained rats may have pushed their performance towards the optimum. 
Studies using acute shock stressors have also found that stress can enhance spatial ability, but the facilitating effects were only seen under specific circumstances such as certain cue conditions, or a specific length of time after the stress (Wade \& Maier, 1986; Healy \& Drugan, 1996; Bowman et al., 2001). This study may have consistently found a facilitating effect of stress because the stressor used was relatively mild.

Restrained rats of both sexes gained less weight than the controls. This effect of stress has been reported in other studies (e.g. Bowman et al., 2001; Faraday, 2002), and may have been exaggerated here because restrained subjects may have had reduced ability to compete for restricted food with their control cage-mate. It is possible that this could account for the improved spatial ability in the restrained rats, as they may have been hungrier and therefore more motivated to find the food rewards. However, stressed rats put on less weight than control rats even when on an unrestricted diet suggesting that stress reduces hunger rather than ability to compete for food (Faraday, 2002). There were also no differences in the number of uneaten reward errors, which may be related to hunger, made by the restrained and control groups. These lines of evidence suggest that it is unlikely that there were any differences in food motivation between the restrained and control rats, but using the Morris water maze, which does not use food motivation, to test spatial ability could prove this. If the restraint stress improves spatial ability via the Yerkes-Dodson law, rather than by affecting motivation, its facilitating effect should still be apparent in the MWM.

To summarise: acute stress tended to improve spatial ability; it did not affect the tendency to make uneaten reward errors; but did decrease activity in female but not male rats. Although explainable, these findings were not predicted by my hypothesis. It is still possible that sex differences in spatial ability, and uneaten reward errors, could be caused by differences in motivation, this possibility will be addressed in chapter 5 . 


\section{Chapter 5: The effects of different levels of food restriction on the performance of male and female laboratory rats in the radial arm maze}

\subsection{Introduction}

Male and female laboratory rats differ substantially in food intake and body-weight. Adult male rats are consistently heavier, and eat more, than females (B\&K Universal Group Catalogue; Slob \& van der Werff ten Bosch, 1975). Therefore, it is possible that the sexes may experience different levels of motivation when experiencing the same level of diet restriction.

Radial arm maze (RAM) studies vary greatly in both the level of, and method used for, food restriction (e.g. Einon, 1980; Maier \& Pohrecky, 1986; Van Haaren et al., 1987; Schenk et al., 1990; Wilkie et al., 1992; Daniel et al., 1999; Fader et al., 1999; Olthof et al., 1999; Boakes et al., 2000). Food restriction regimes usually involve one of two methods (Hurwitz \& Davis, 1983): either the subjects are given ad libitum access to food for a limited amount of time, for example one hour a day; or the subjects have target weights calculated as a percentage of the body-weight of an unrestricted control group - in this case they are weighed regularly and their food intake is adjusted to allow them to meet their target weights. The target weight method is more commonly used, but even within this method there is variation in the percentage body-weight used to calculate the target weight. A review by Weinstock (1972) suggests that different food restriction methods can affect the results of behavioural experiments.

Van Hest et al. (1988) looked for motivational differences between male and female rats exposed to progressive ratio schedules of reinforcement. In such schedules the subject is required to make an increasing number of responses in order to obtain food rewards. The maximum number of responses that a subject will make is an indicator of their motivation. Although Van Hest et al. (1988) were unable to find any 
differences between male and female rats, they only tested subjects at a single level of food restriction ( $80 \%$ free-feeding body-weight). This level is unusually strict when compared to the $85-90 \%$ restriction levels commonly used in RAM studies. It is possible that other levels of food restriction and different tasks may affect the sexes differently.

If different levels of food restriction do affect performance on an appetitivelymotivated task the effect is probably mediated via the level of motivation to perform the task (reviewed in Weinstock, 1972; Toth \& Gardiner, 2000). Increased food restriction is expected to increase motivation and improve performance on the cognitive task because the subject is motivated to obtain greater or faster food rewards in order to assuage its hunger. However, motivational state may have different effects on different measures of spatial performance. For example, psychologists generally agree that there is a trade-off between speed and accuracy such that increased speed results in reduced accuracy and vice versa (e.g. Fitts, 1966; Lachman et al., 1979). A study testing several strains of rats in a water maze has also reported this trade-off. Strains of rats that swam slower tended to find the platform more accurately than faster swimming strains (Andrews et al., 1995). If such a trade-off exists in the RAM then increased speed may result in a decrease in accuracy and therefore less food reward. Under such circumstances increased food restriction may be expected to increase accuracy (and therefore reward) by decreasing speed.

Several studies have found the expected positive correlation between food restriction and cognitive performance: Vawter and Van Ree (1989) found that male Wistar rats with a higher level of food restriction (22.2\% weight loss, as opposed to $16.4 \%$ ) performed better on a spatial task where the subjects were trained to remember the location of food hidden in holes in the floor of an arena. Gaulin and Wartell (1990) showed that prairie voles (Microtus ochrogaster) deprived of food for $24 \mathrm{hr}$ prior to a symmetrical maze task (similar to the Davenport maze; figure 1.2.c) made fewer errors than voles that only had $15 \mathrm{hr}$ of food deprivation. They tested both males and females but did not find any sex by deprivation interactions. Timberlake and White 
(1990) compared $85 \%$ food deprived female rats with a non-deprived group on a RAM working memory task. They found that the deprived rats performed better than the non-deprived rats, however the maze contained no food rewards and so this finding may have more to do with motivation to explore the maze, rather than simply to find food. Similarly, Haga (1995) found that food deprived male rats made fewer errors in a rewarded RAM working memory task than non-deprived rats.

A couple of studies have not found an effect of increasing food deprivation on cognitive performance, although both used non-rodent species. Healy and Cleland (submitted) gave coal tits (Parus ater) and great tits ( $P$. major) a delayed nonmatching to sample task after 0,2 or $4 \mathrm{hr}$ of food deprivation. They found that deprivation duration did not affect spatial performance of either species, but the coal tits completed more trials as deprivation duration increased. Meyer (1951) found that increasing periods of food deprivation $(1,23$ and $47 \mathrm{hr})$ did not affect rhesus monkeys' activity or performance on a discrimination-learning task.

In this experiment I looked at the effects of sex and food deprivation on a reference memory task in a RAM, using laboratory rats. I hypothesised that subjects with stricter food restriction would perform better, and that this effect would be more noticeable in females because of their smaller body mass. Therefore, sex differences in spatial ability would be more likely to be found when less severe food restriction was used. Half of the subjects of each sex were put on restricted diets at $95 \%$ of their free-feeding weight and half were kept at $85 \%$ of their free-feeding weight. This resulted in four groups of subjects, namely, 'male strict diet', 'male moderate diet', 'female strict diet', and 'female moderate diet'. This also provided the opportunity to look for any effects that the different levels of food restriction might have on the uneaten reward errors previously observed in the RAM (chapters 2 and 4).

Subjects were tested in a black-white box prior to starting training on the maze in order to see whether there were differences in stress response between the four groups, as this is a possible way that food deprivation could affect performance in the maze (Galea et al., 1994c; Kavaliers et al., 1996; Perrot-Sinal et al, 1996). The black-white box is a commonly used behavioural test of anxiety (Crawley \& 
Chapter 5: The effects of food restriction on rats' radial maze performance

of a brightly illuminated white compartment linked to a dark, black compartment. The subject is placed within the box and its behaviour is observed. The subject can be described as having two conflicting motivations: exploring the whole box versus avoiding the white side. The amount of time spent in the white side is positively correlated with the subjects' anxiety levels (e.g. Bilkei-Gorzó et al., 1998; Henniger et al., 2000). Previous work carried out in my lab has found that control female rats display less anxious behaviour than males in the black-white box, and that it is also sensitive to restraint stress, with restrained animals showing more anxious behaviour than controls (Beveridge, 2001).

\subsection{Materials and methods}

The experiment was carried out during October-December 2001. The maze room was the same as that described in chapter 2 except that the water maze had been removed. The RAM was placed on a wooden platform raised $400 \mathrm{~mm}$ off the floor in the middle of the room (figure 5.1).

Figure 5.1: The radial arm maze set-up.

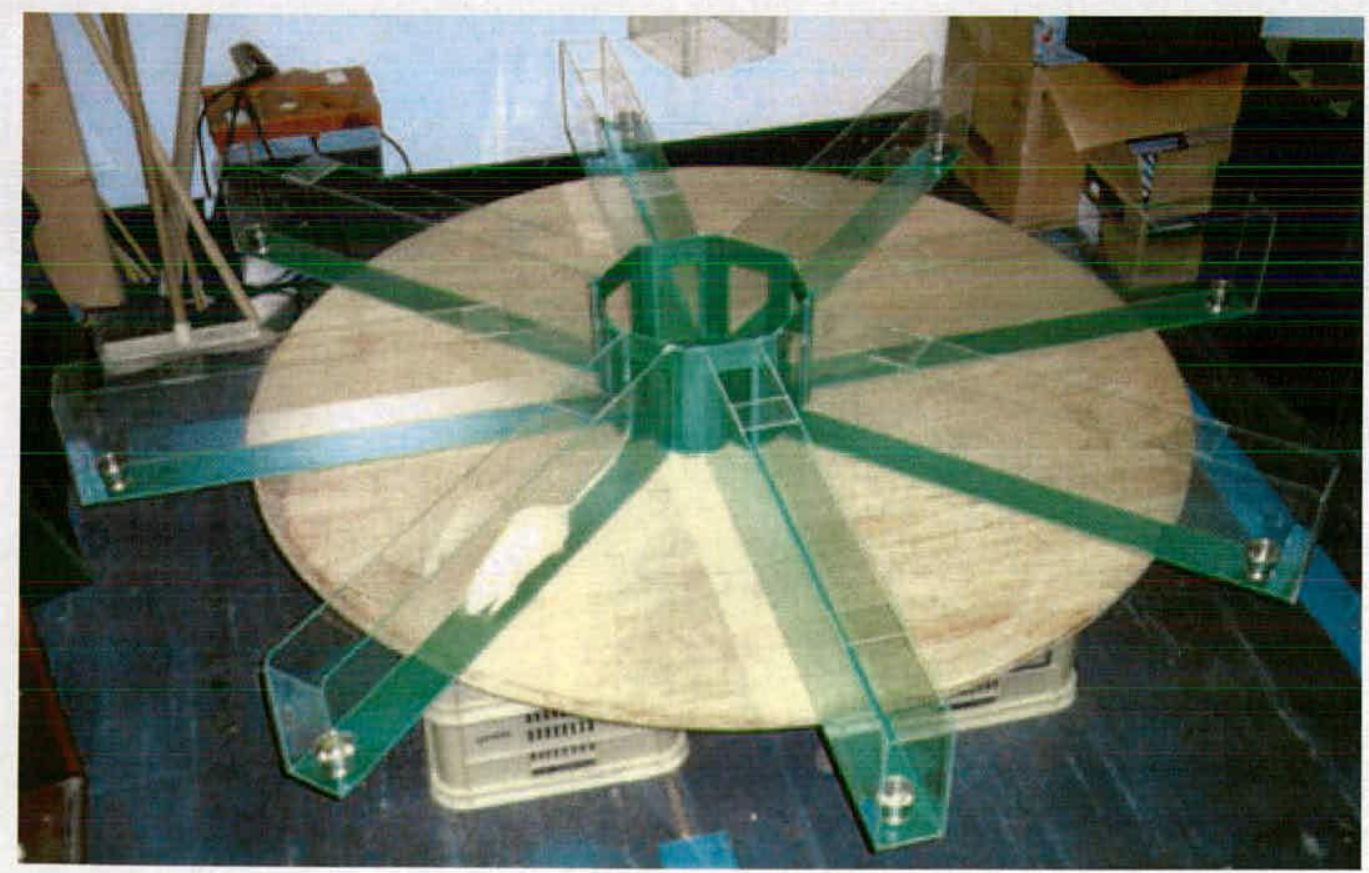


Subjects were 12 male and 12 female experimentally naïve Sprague-Dawley rats, weighing $175-200 \mathrm{~g}$ when supplied by B\&K Universal Ltd. They were housed in same-sex pairs in the animal house under the conditions described in chapter 2. Half of the female and half of the male cages were randomly assigned to the strict diet group; the remaining cages were assigned to the moderate diet group.

\section{Food restriction}

Four days after arriving in the animal house the subjects were weighed and put on a food restriction regime aimed at keeping them at $85 \%$ (strict group) or $95 \%$ (moderate group) of their free-feeding weight, compared to weight-gain charts from B\&K (B\&K Universal Group Catalogue). Target weights for the next week were calculated by adding a growth allowance calculated from the supplier's growth curves to $85 \%$ or $95 \%$ of the subjects' initial weight. They were weighed every day during the first fortnight and the amount of food provided was adjusted as necessary. For the second week the target weights were calculated by adding the next week's growth allowance onto the previous target weight, but on subsequent weeks the target weights were calculated by adding the growth allowance onto the subjects' present weight. This gave the rats two weeks to reach their target weights, but once the experiment was started it meant that all the rats within a group were similarly motivated as they all had the same difference between their actual weight at the start of the week and their target weight for the end of the week. During the experiment subjects were weighed three times a week in the morning before maze training, and fed in the afternoon on return to the animal house after the day's trials. Any food remaining the next morning was removed prior to starting the experiment.

\section{Black-white box}

Two weeks after food restriction began the subjects were tested in the black-white box. This consisted of an open-topped rectangular wooden box (figure 5.2), divided into two equal sized compartments by a board that had an archway cut into it to allow free access between the sides. One half of the box was painted black and the 
other white. An angle-poise lamp with a 40 watt bulb was positioned over the centre of the white side, which was the only source of illumination in the room.

Figure 5.2: The black-white box.

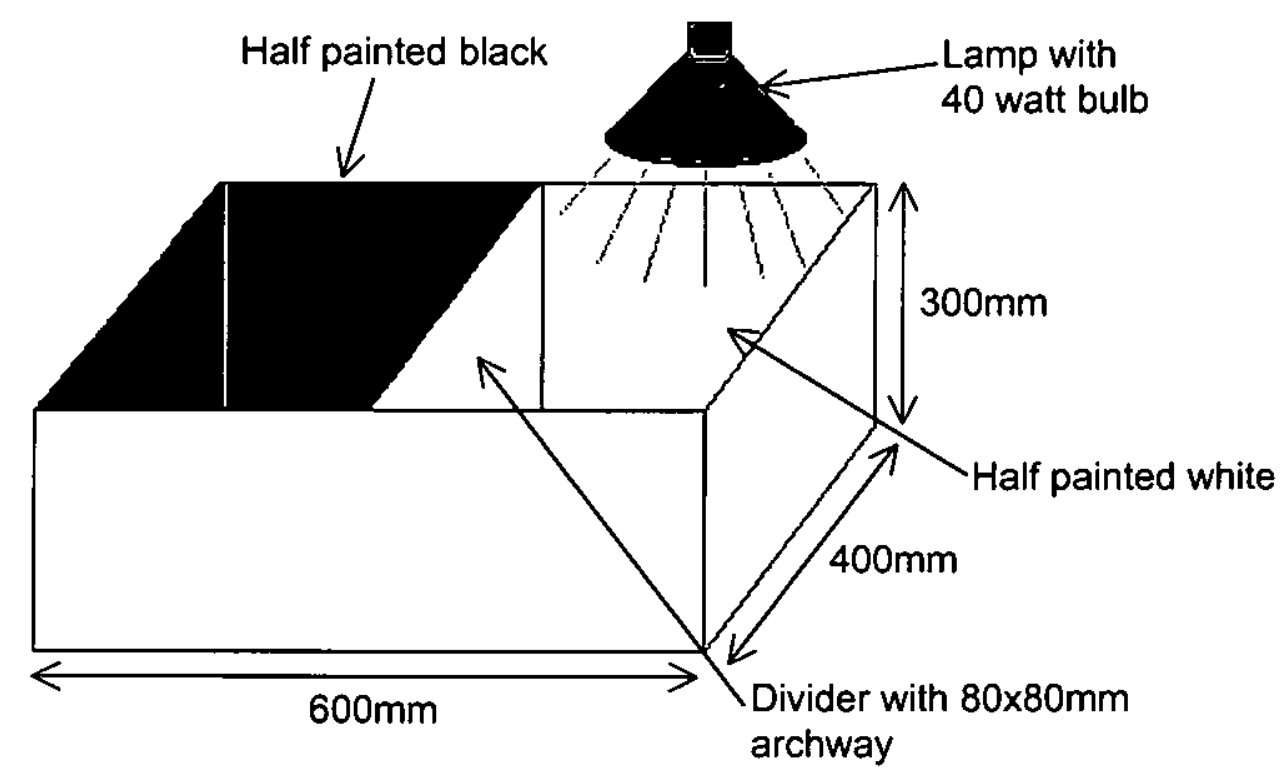

Testing began at 1130. Prior to testing the box was soiled by two rats that were not used in the experiment in order to reduce any effects of order. The experimental rats were placed in the box in a pseudorandom order, whereby each group of four trials within a day (i.e. the first four, trials 5-8, trials 9-12) included one subject from each of the four treatment groups. The order of the treatment groups within each set of four trials, and the order of subjects within a group was fully randomised. Each rat was placed in the centre of the black side facing away from the archway, and its behaviour was recorded on videotape for $5 \mathrm{~min}$. Faeces were removed between trials, but otherwise the box was not cleaned.

The total time that each subject spent in the white side and the number of crossings that it made between sides (a crossing was defined as more than three-quarters of the subject's body passing through the archway) were recorded from the videotapes. 
Chapter 5: The effects of food restriction on rats' radial maze performance

Each parameter was recorded on three separate occasions by the same experimenter, and the mean calculated.

\section{Pretraining in the RAM}

The RAM used in this experiment has already been described in section 2.2.2. For four days before pretraining started the subjects were given four of the food rewards (Kellogg's' Honey Loops) per cage and one eggcup was placed in each cage. This was done in order to allow the subjects to get used to these novel features of the maze. The first pretraining session started in the afternoon after black-white box testing (1500), but on subsequent days pretraining started at approx 1100 .

Each subject was placed, in a pseudorandom order (as described above), in the fully rewarded maze for $5 \mathrm{~min}$ or until they had eaten all of the rewards, once a day for four days. The maximum time in the maze was then increased to $15 \mathrm{~min}$ for a further eight days. After this time two subjects (one moderate diet male and one strict diet female) were still not eating all of the rewards within $15 \mathrm{~min}$. These subjects were given extra pretraining trials on a day when the rest of the subjects did not go into the maze. The moderate diet male took four extra trials and the strict diet female took two extra trials before they ate all of the rewards within $15 \mathrm{~min}$.

\section{Experiment}

The experiment was started on the day immediately after pretraining finished (four weeks after food restriction was started). The rats were given a reference memory task with four out of eight arms rewarded. The basic procedure was the same as that detailed in section 2.2.2. Each subject was given one trial a day, seven days a week, for 25 days. The trials started at approx 1230. The order in which the subjects were placed in the maze was pseudorandomised in the same way as for the black-white box test. 


\subsection{Results and analysis}

All analysis was carried out using Minitab. In each case the data were checked to see if they met general linear model (GLM) assumptions and transformations were applied as appropriate. Non-significant interactions were removed from the models.

\section{Weights}

The following GLM, equivalent to a 2-way analysis of variance (ANOVA), was applied to the subjects' weights at the beginning and end of the experiment:

Response $=\operatorname{sex}+\operatorname{diet}+\operatorname{sex} * \operatorname{diet}$

The interaction was non-significant for the weights at the start of the experiment and was left out of the final model. There was a significant difference between the male and female rats' weights before food restriction was started (week 1), but no difference between the two diet groups within each sex (figure 5.3: $\operatorname{sex} F_{1,21}=18.97$, $\mathrm{p}<0.001 ; \operatorname{diet} \mathrm{F}_{1,21}=0.19, \mathrm{p}=0.666$ ).

At the end of the experiment (week 8) the sex by diet interaction was significant $\left(\mathrm{F}_{1,20}=4.96, \mathrm{p}=0.038\right)$. Tukey simultaneous tests showed that there was a significant difference between the male strict diet and male moderate diet groups, but not between the two female groups (male moderate versus male strict $\mathrm{t}=-3.63, \mathrm{p}=0.008$; female moderate versus female strict $\mathrm{t}=-0.48, \mathrm{p}=0.963$ ). The male strict diet group weighed less than the male moderate diet group (figure 5.3).

I noticed that sometimes a cage of rats did not finish their food allowance. As whether or not the subjects were satiated at the start of the experiment could affect their motivation I decided to test whether there were differences in this behaviour between groups. I calculated the percentage of days on which each cage of rats finished their food allowance. As there were only three cages in each group a nonparametric Kruskal-Wallis test was used to test for differences between the groups. There were significant differences between the groups $(H=8.60, p=0.035$, adjusted for ties). The female moderate diet group finished their food on fewer days than the other three groups (figure 5.4). 
Chapter 5: The effects of food restriction on rats' radial maze performance

Figure 5.3: Mean weights of the four groups of rats during the experiment $+1-$ standard error of the mean (SEM). $n=6$ per group.

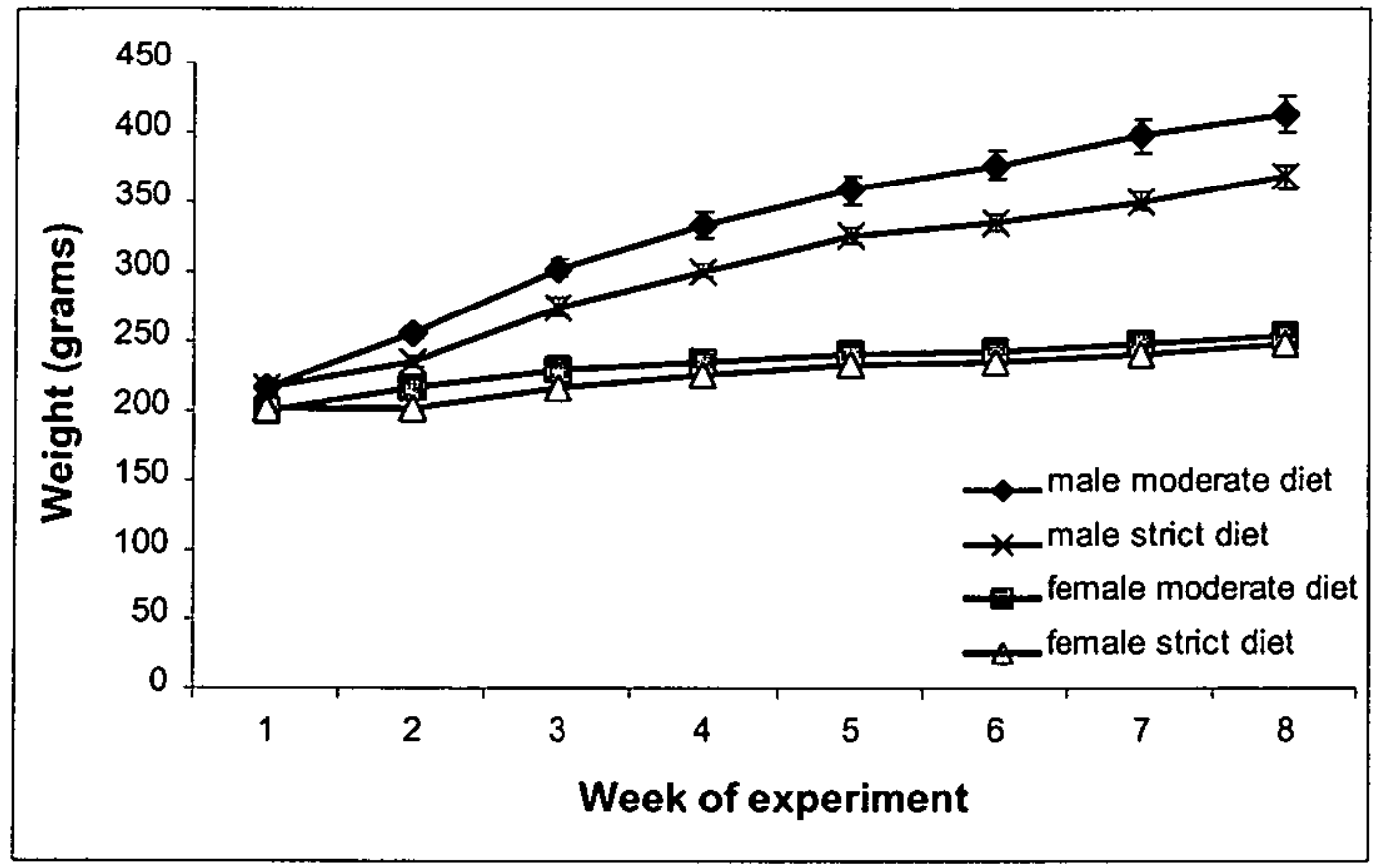

Figure 5.4: Percentage of days on which the cages of rats finished their food allowance $+/$ - SEM. $n=3$ per group.

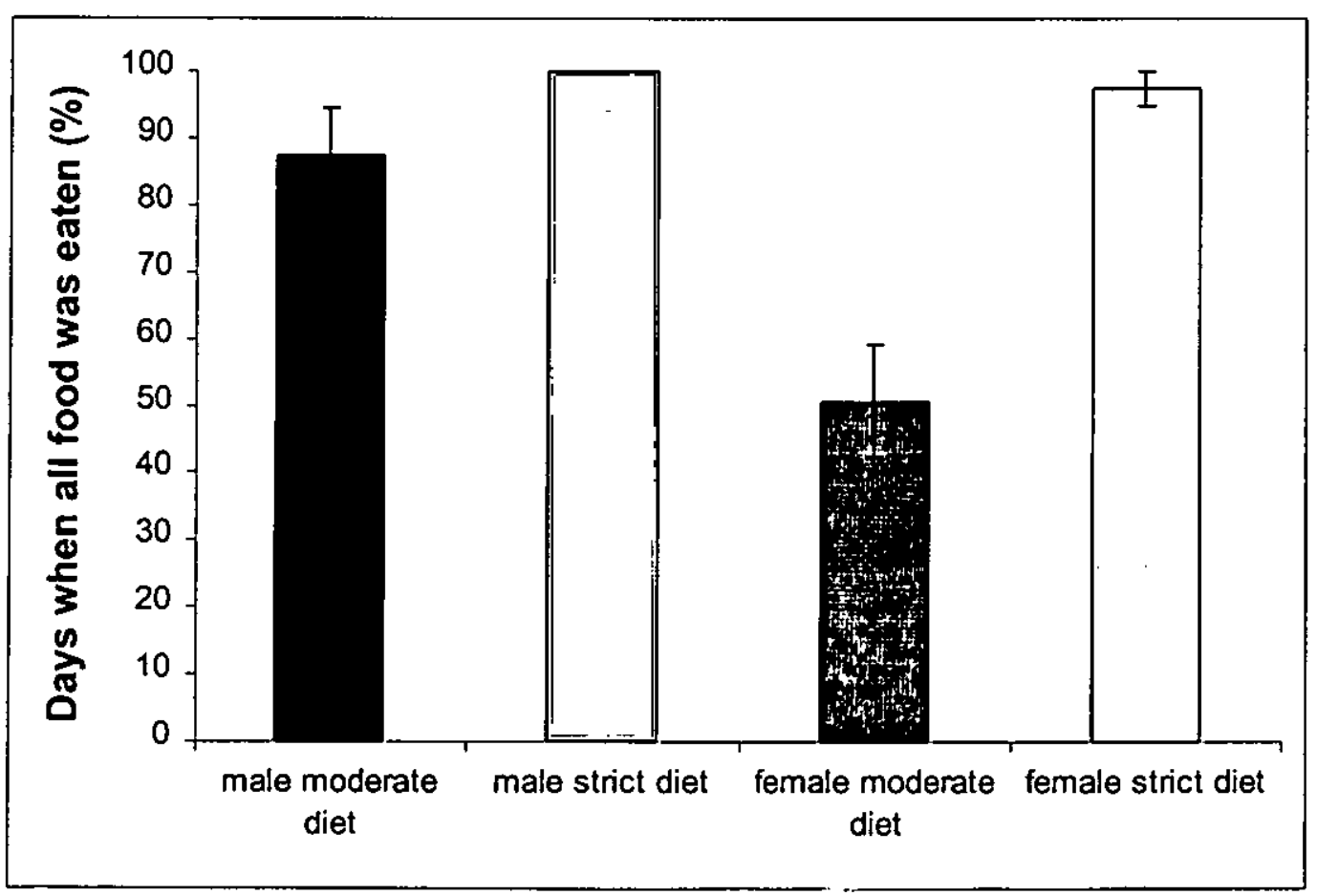




\section{Black-white box}

The following GLM was used to test for effects of sex and diet on total time that each subject spent in the white side and the number of crossings that it made between sides in the black-white box:

Response $=\operatorname{sex}+\operatorname{diet}+\operatorname{sex}{ }^{*} \operatorname{diet}$

The interaction term was not significant for the number of crossings was excluded from the final analyses. Sex had a significant effect $\left(F_{1,21}=5.70, p=0.026\right)$. Males made fewer crossings than females (figure 5.5.a). The main effect of deprivation was approaching significance $\left(\mathrm{F}_{1,21}=4.25, \mathrm{p}=0.052\right)$. Subjects on the strict diet tended to make fewer crossings than subjects on the moderate diet (figure 5.5.a).

The interaction term was approaching significance for the amount of time spent on the white side $\left(\mathrm{F}_{1,20}=3.09, \mathrm{p}=0.094\right)$. Tukey simultaneous tests showed that the male strict diet group spent less time on the white side than either of the female groups (versus female strict $t=3.139, p=0.0245$; versus female moderate $t=3.195, p=0.0217$; figure 5.5.b). 
Chapter 5: The effects of food restriction on rats' radial maze performance

Figure 5.5: Behaviour during $5 \mathrm{~min}$ in the black-white box $+/$ - SEM. $n=6$ per group.

a) Number of crossings from one side to the other.

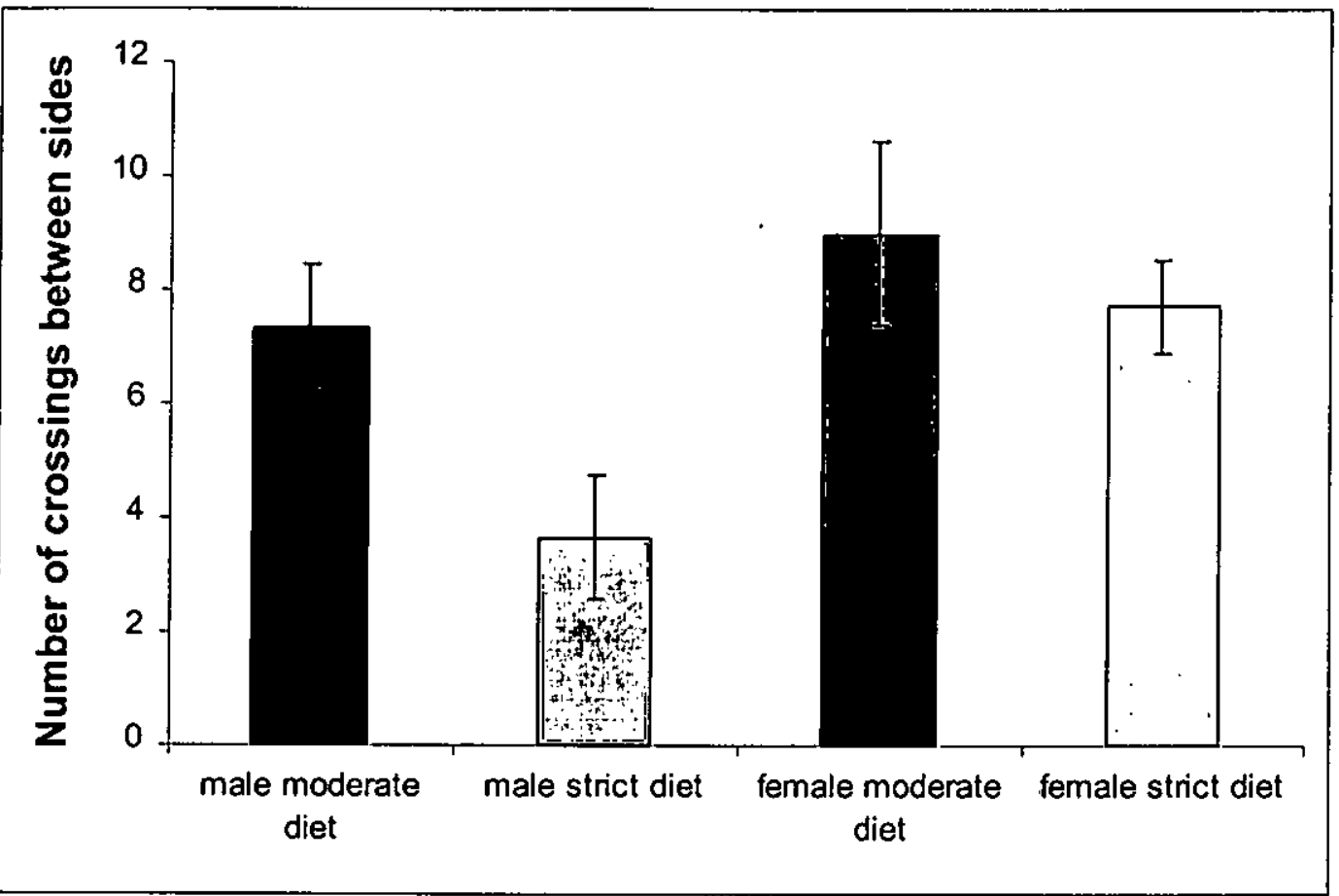

b) Time spent in the white side of the black-white box.

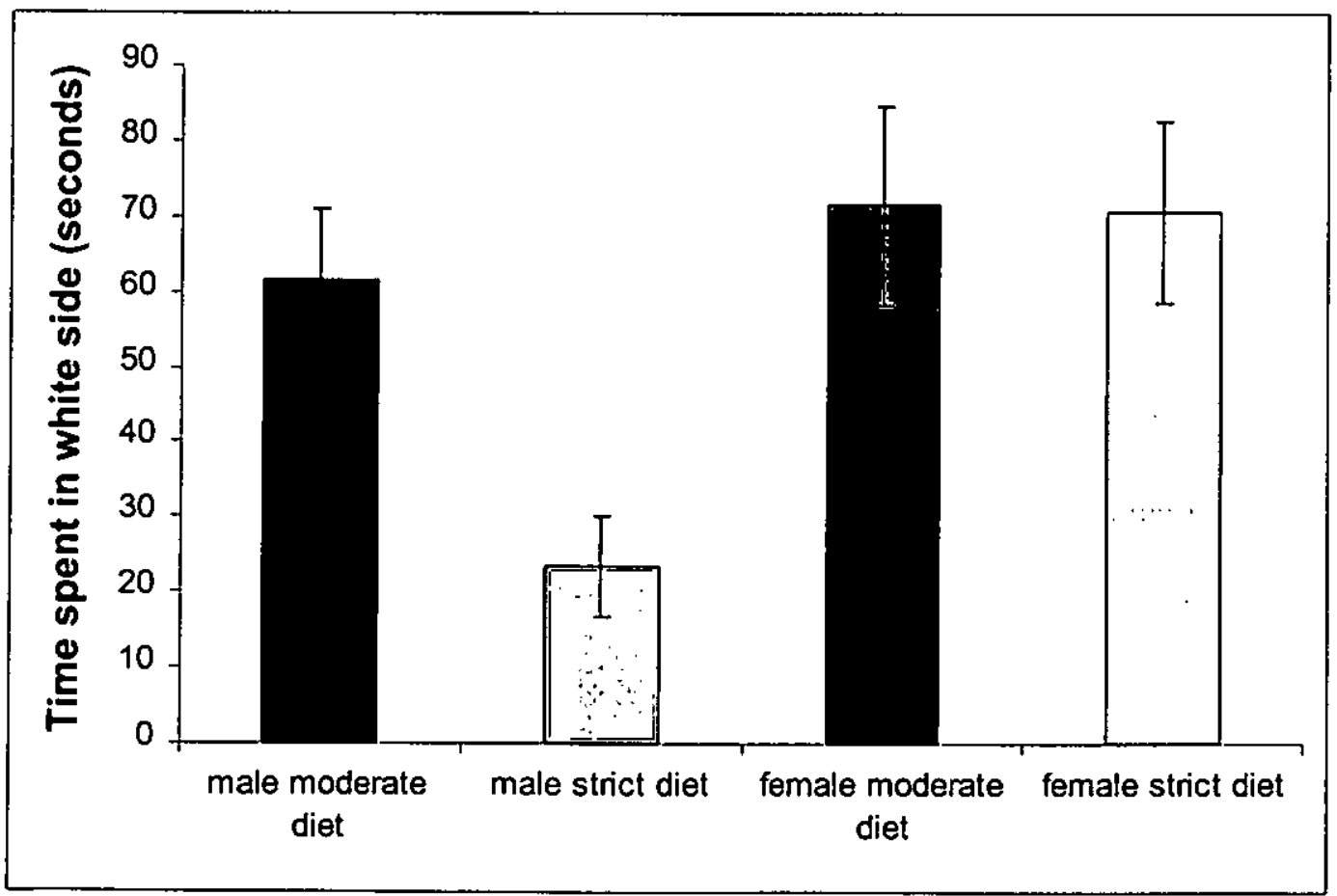




\section{RAM repeated-measures analysis}

The following parameters were calculated for each RAM trial:

1. 'Time per arm': the time in seconds that the subject took to eat all of the rewards in the maze divided by the number of arms that it entered.

2. 'Working memory errors': the number of times that the subject entered an arm that it had already visited during that trial, which did not contain a food reward.

3. 'Reference memory errors': the number of first entries into an arm that never contained a food reward.

All data were blocked by taking the mean of every five trials. The following GLM, equivalent to a repeated-measures ANOVA with two between-subjects effects (sex and diet) and one within-subject effect (block) was used:

Response $=\operatorname{sex}+$ diet + sex $*$ diet + rat $($ sex diet $)+$ block + block $*$ sex + block*diet + block*sex*diet

The 'rat(sex diet)' mean square was used as the error term to calculate the F-ratios for the between-subjects effects.

The block by sex by diet interaction was significant for time per arm (Figure 5.6.a $\mathrm{F}_{4,80}=2.77, \mathrm{p}=0.033$ ). Tukey simultaneous tests showed that this was due the female moderate group taking significantly less time per arm on the first two blocks than either the male moderate or the female strict groups (block 1 versus male moderate $t=-4.17, p=0.011$; versus female strict $t=4.28, p=0.008$ : block 2 versus male moderate $t=4.27 \mathrm{p}=0.008$; versus female strict $\mathrm{t}=4.185 \mathrm{p}=0.010$ ). None of the other block interactions were significant for any measure and so they were excluded from the final analyses.

The main effect of block was significant for both working and reference memory errors (table 5.1). Fewer working and reference memory errors were made as the experiment progressed (figure 5.6.b\&c). The sex by diet interaction term was 
Chapter 5: The effects of food restriction on rats' radial maze performance

significant for reference memory errors (table 5.1). Tukey simultaneous tests showed that the female strict group made more reference memory errors than both the female moderate and male strict groups (versus female moderate $t=4.09$, $\mathrm{p}=0.001$; versus male strict $\mathrm{t}=2.93, \mathrm{p}=0.022$ ). Neither the sex by diet interaction nor the main effect of diet were significant for working memory errors (table 5.1).

Table 5.1: F-ratios for the repeated-measures ANOVAs. Degrees of freedom for sex, restraint, and sex ${ }^{\star}$ restraint $=1,20$; for block $=4,92 .{ }^{*}=p<0.1{ }^{* *}=p<0.05$, $\star \star \star x=p<0.01$. Working memory errors were square-root transformed for both analyses.

\begin{tabular}{|c|c|c|c|c|c|c|c|c|}
\hline \multirow[t]{2}{*}{ Response } & \multicolumn{4}{|c|}{ With uneaten reward errors } & \multicolumn{4}{|c|}{$\begin{array}{l}\text { Without uneaten reward } \\
\text { errors }\end{array}$} \\
\hline & sex & diet & sex*diet & block & sex & diet & sex*diet & block \\
\hline $\begin{array}{l}\text { Reference } \\
\text { memory } \\
\text { errors }\end{array}$ & 0.04 & 1.54 & $6.11^{\star \star \star}$ & $10.94^{\star \star \star}$ & 0.01 & $3.41^{\star}$ & $7.50^{\star \star}$ & $7.01^{\star \star}$ \\
\hline $\begin{array}{l}\text { Working } \\
\text { memory } \\
\text { errors }\end{array}$ & 0.01 & 0.08 & 1.17 & $7.47^{\star \star \star}$ & 1.17 & 1.01 & 2.57 & $3.31^{\star}$ \\
\hline
\end{tabular}

In previous experiments any sex differences were most apparent at the beginning of the training (chapters $2 \& 4$ ), and so statistical tests were carried out on the first block of data for working and reference memory errors using a 2-way ANOVA (effects of sex and diet). Working memory errors were square root transformed. The interaction was not significant for either measure. There were no significant effects of sex or diet on the number of working or reference memory errors made during the first block (reference -memory errors: sex $F_{1,21}=0.14, p=0.717$; diet $F_{1,21}=2.73$, $p=0.113$; working memory errors: $\operatorname{sex} F_{1,21}=0.17, p=0.685$; diet $F_{1,21}=0.02, p=0.881$ ). 
Figure 5.6: Performance of the four groups of rats in the eight-arm radial arm maze with four rewarded arms +/- SEM. One block is the mean of five trials. $N=6$ for each group.

a) Time per arm (total time divided by number of arms entered).

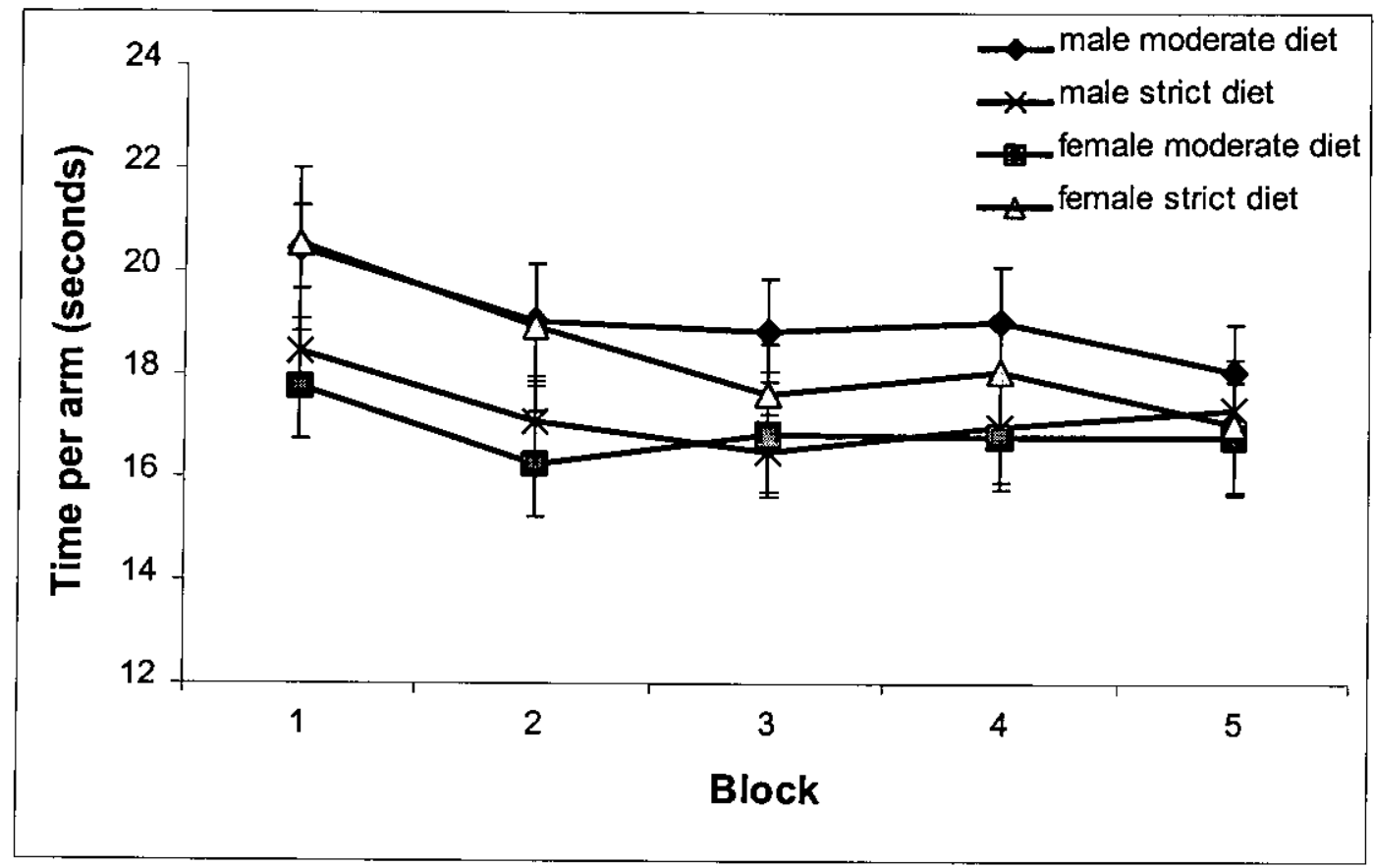

b) Reference memory errors (entries into arms that never contain food rewards).

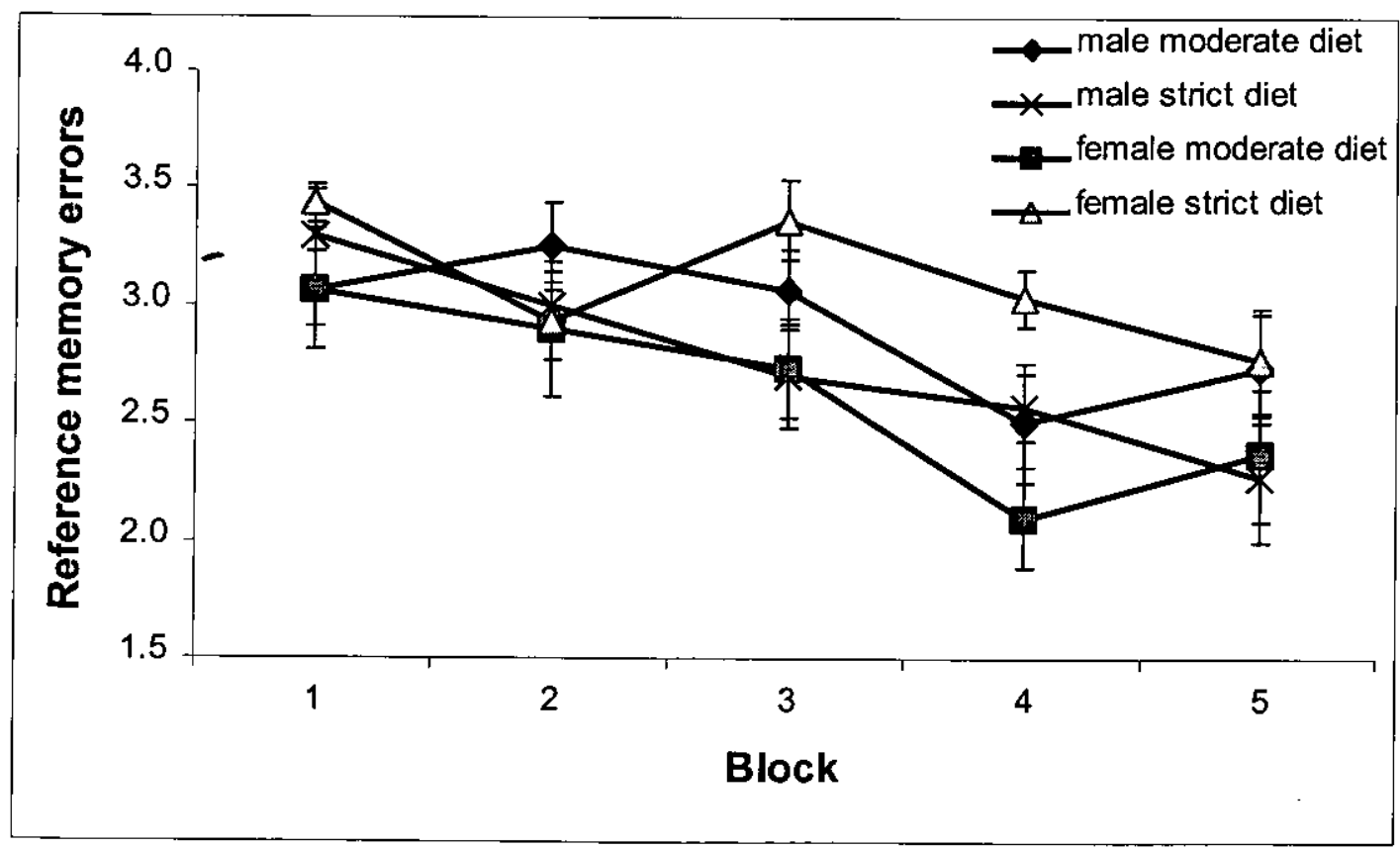


Chapter 5: The effects of food restriction on rats' radial maze performance

c) Working memory errors (entries into unrewarded arms previously visited on that trial).

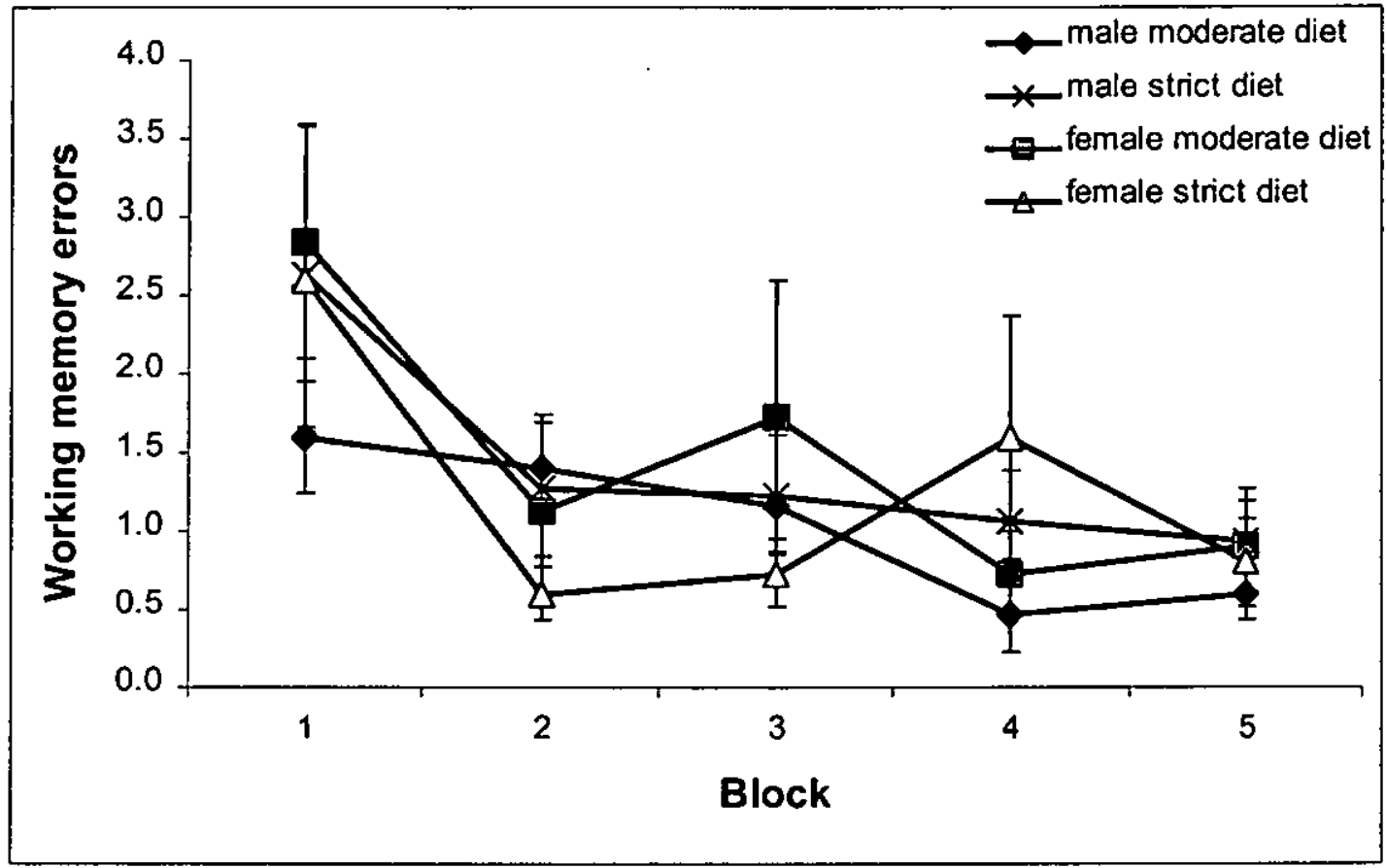

\section{RAM uneaten reward errors}

As the uneaten reward errors data did not meet the GLM assumptions they were analysed using non-parametric tests. Separate Mann-Whitney tests for the effects of sex and diet were performed on the mean number of uneaten reward errors made by each rat during all 25 trials. There was no statistically significant effect of either sex or diet on the mean number of uneaten reward errors made (figure 5.7; sex $\mathrm{W}=122.5$, $\mathrm{p}=0.1074$; diet $\mathrm{W}=144.5, \mathrm{p}=0.7656$, both adjusted for ties).

In previous RAM experiments the female subjects had made more uneaten reward errors than males, and this effect was particularly noticeable at the beginning of the experiment (figures $2.4 \& 4.4$ ). Therefore, separate Mann-Whitney tests for the effects of sex and diet were carried out on the first block. The results from these tests were also non-significant $(\operatorname{sex} \mathrm{W}=128.5, \mathrm{p}=0.2057$; diet $\mathrm{W}=141.0, \mathrm{p}=0.6085$, both adjusted for ties). 
Figure 5.7: Uneaten reward errors made by the four groups of rats in the RAM +/SEM. One block is the mean of five trials. $N=6$ for each group.

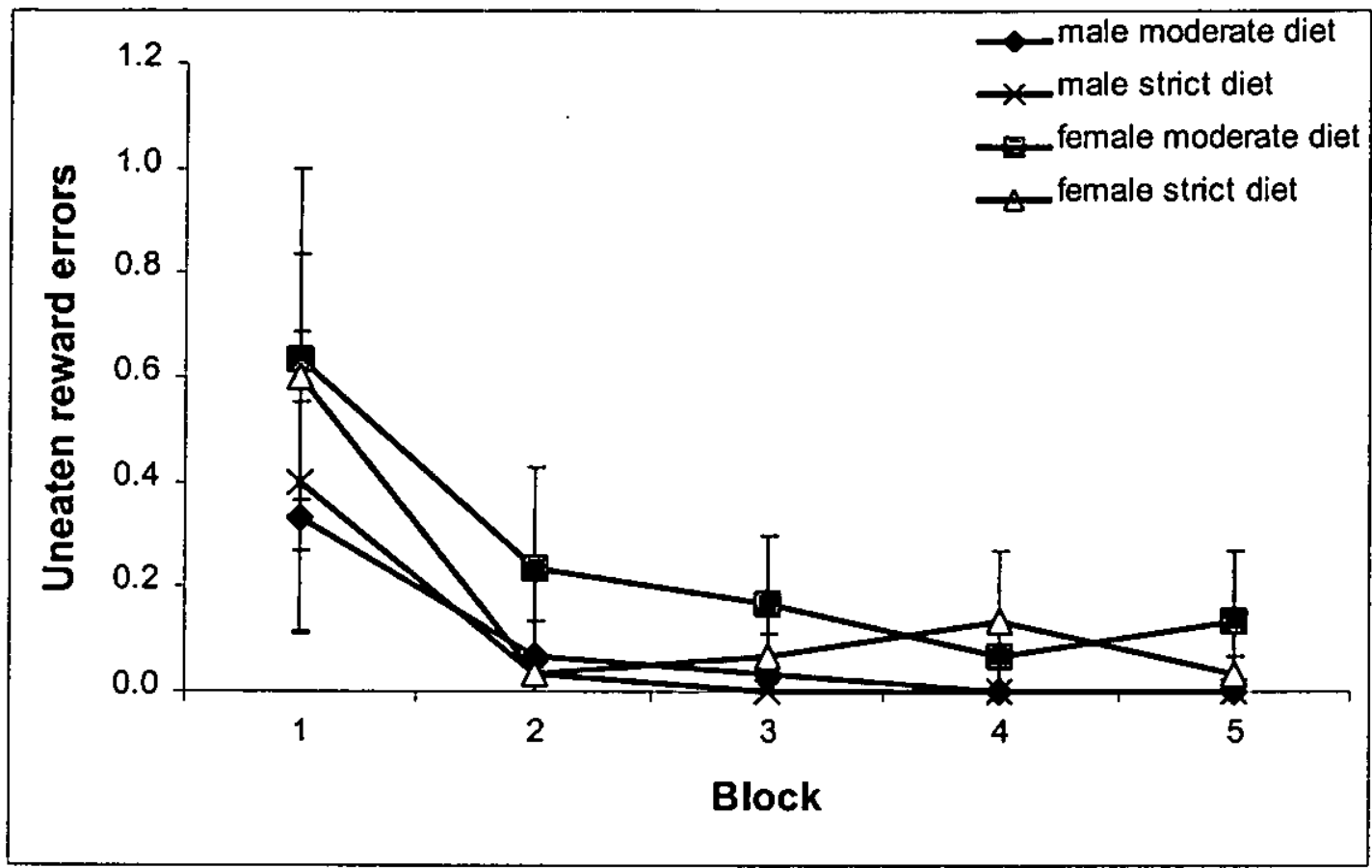

\section{RAM analysis excluding uneaten reward errors}

As in previous chapters, the uneaten reward errors were removed from the raw data and the number of working and reference memory errors (figure 5.8) were reanalysed with repeated-measures ANOVAs. As in the initial analysis none of the interaction terms involving block were significant and so were left out of the final analysis. The only change in significance between the analyses was for the working memory errors block term, which became non-significant when the uneaten reward errors were removed (table 5.1). 
Figure 5.8: Spatial performance excluding uneaten reward errors in the eight arm RAM for the four groups of rats +/- S.E.M. One block is the mean of five trials. $N=6$ for each group.

a) Reference memory errors (entries into arms that never contain food rewards).

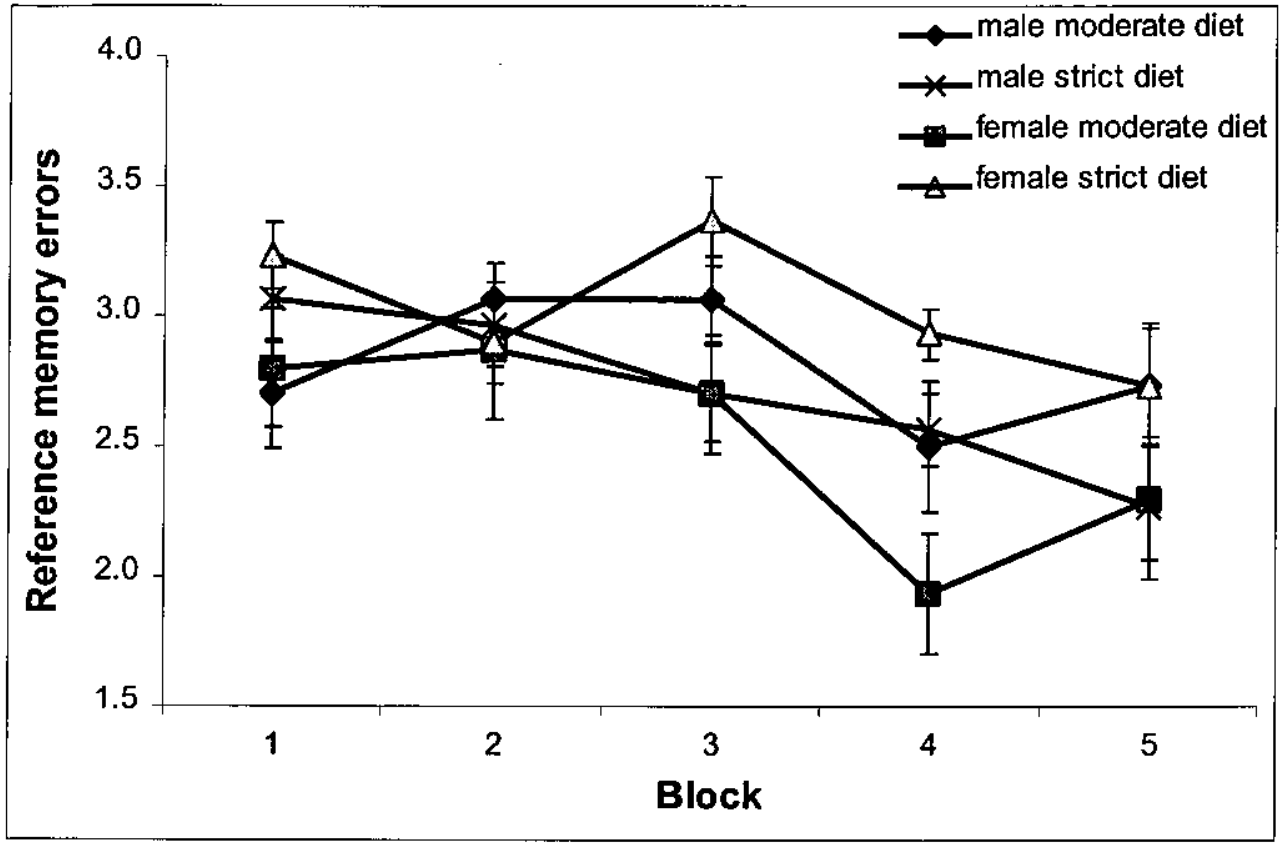

b) Working memory errors (entries into arms previously visited on that trial).

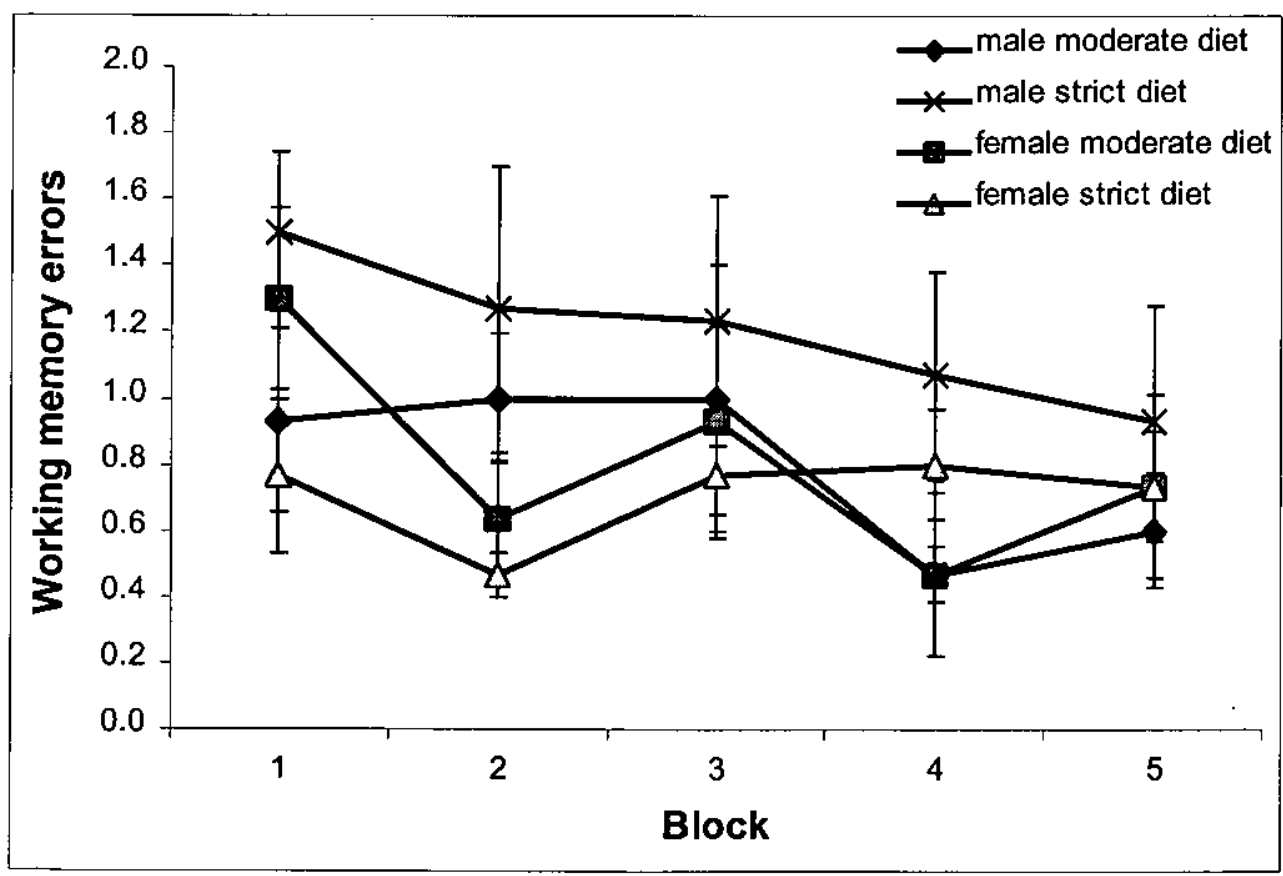


The first blocks were again analysed for effects of sex and diet. No transformations were necessary. The interaction was not significant for reference memory errors and so was removed from the model. The effect of sex was not significant, but the effect of diet was ( $\operatorname{sex} F_{1,21}=0.53, p=0.475$; diet $\left.F_{1,21}=4.76, p=0.041\right)$. The rats on the strict diet made more reference memory errors than the subjects on the moderate diet (figure 5.8.a). The sex by diet interaction was significant for working memory errors $\left(\mathrm{F}_{1,20}=4.60, \mathrm{p}=0.044\right)$, and although none of the Tukey tests were significant the biggest difference was between the male strict and female strict groups, with the latter making fewer working memory errors (figure 5.8. $\mathrm{b} ; \mathrm{t}=-2.023, \mathrm{p}=0.213$ ).

\section{Regression analysis}

As in the previous RAM studies the number of trials taken to reach criterion during pretraining, weight gain during the period of food restriction, and mean time per arm over all 25 trials were regressed against the mean number of uneaten reward errors made by each subject over all 25 trials. The following GLM was used:

\section{Uneaten reward errors $=$ sex + diet + covariate}

Uneaten reward errors were square-root transformed for all analyses. None of the covariates had a significant effect on the number of uneaten reward errors made when sex and diet were taken into account (pretraining $\mathrm{F}_{1,20}=2.57, \mathrm{p}=0.125$; weight gain $\mathrm{F}_{1,20}=2.25, \mathrm{p}=0.149$; time per arm $\mathrm{F}_{1,20}<0.01, \mathrm{p}=0.986$ ). These results were different to those found in the previous RAM experiments; in the first experiment pretraining had a significant effect and time per arm had an effect approaching significance (section 2.2.3), and in the experiment described in chapter 4 weight gain had an effect approaching significance (section 4.3).

As this experiment examined the effects of food deprivation on spatial performance, working and reference memory errors (with uneaten reward errors removed) were regressed against weight gain, allowing for sex and diet. This tested whether different weight gains within each group were related to spatial performance. Weight gain had a significant effect on the number of reference memory errors made $\left(F_{1,20}=13.16, p=0.002\right)$. Rats that gained more weight within a group tended to make 
Chapter 5: The effects of food restriction on rats' radial maze performance

more errors (figure 5.9). There was no effect of weight gain on the number of working memory errors made $\left(\mathrm{F}_{1,20}=0.22, \mathrm{p}=0.644\right)$.

Figure 5.9: Regression plot of weight gain during the experiment against mean number of reference memory errors made.

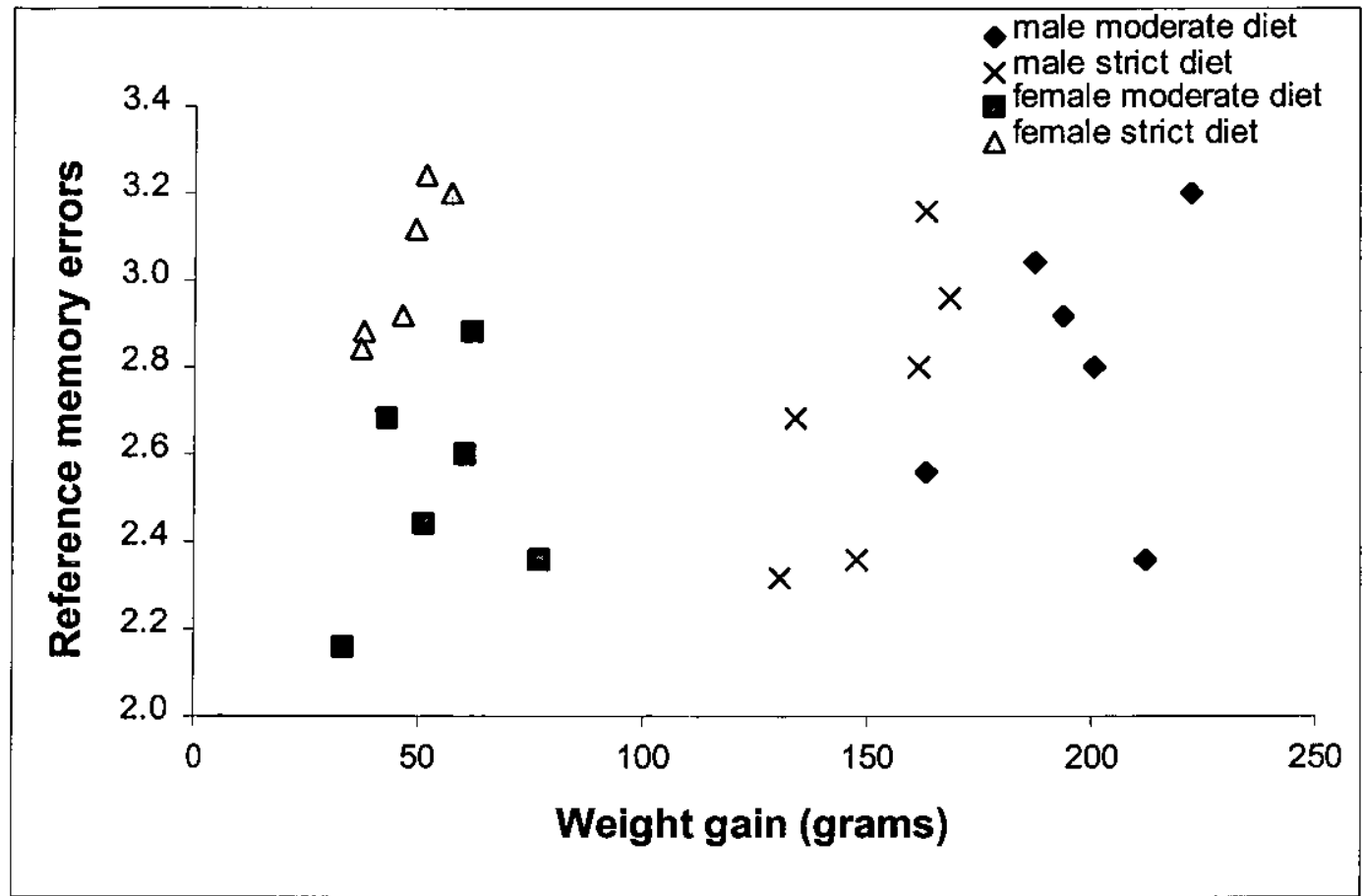

The data from the previous two RAM experiments were also tested for a correlation between spatial ability and weight gain, there were no significant effects (first experiment: weight gain effect on reference memory allowing for sex $F_{1,17}=0.37$, $\mathrm{p}=0.552$; effect on working memory $\mathrm{F}_{1,17}=0.13, \mathrm{p}=0.727$ : restraint stress experiment: weight gain effect on reference memory allowing for sex and restraint $F_{1,20}=1.61$, $\mathrm{p}=0.218$; effect on working memory $\mathrm{F}_{1,20}<0.01, \mathrm{p}=0.965$ ). 


\subsection{Discussion}

Female rats on the strict diet ( $85 \%$ free-feeding body weight) made significantly more reference memory errors than female rats on the moderate diet $95 \%$ freefeeding body weight), an effect that was in the opposite direction to that predicted, and resulted in a sex difference in spatial ability in the rats on the strict diet. Diet did not affect the number of reference memory errors made by the male rats. A similar effect was seen in the number of working memory errors made on the first block, but not throughout the experiment. These results may have been due to the strict food restriction regime directly affecting the females' cognitive ability, rather than motivation to perform the task as I had expected.

Telegdy and Cohen (1971) also found that increased deprivation resulted in reduced cognitive ability. They deprived two groups of male rats of water: one group on a moderate ( $18 \mathrm{hr}$ a day) and one on a strict ( $21 \mathrm{hr}$ a day) deprivation schedule. The subjects were then given a simultaneous discrimination task rewarded with sucrose solution. They were trained to discriminate between two rectangular shapes: either black horizontal versus white vertical, or white horizontal versus black vertical. There were no differences between the groups in the acquisition rate of this task. However, when the subjects were tested with novel pairs of stimuli such as black horizontal versus white horizontal, it was found that the moderately deprived rats were better able to remember and utilize the multiple cue dimensions that had been present in the original task than the strictly deprived subjects.

Telegdy and Cohen's (1971) findings provide an explanation for my results when combined with two facts: first, good reference memory requires rats to remember the location of individual arms relative to cues outside the maze, whilst good working memory performance can be achieved using internal maze cues or a response chain to remember which arms have previously been visited on that trial; second, female rats are known to rely more on landmark cues to solve mazes than males (Williams et al., 1990; Kanit et al, 1998a; Roof \& Stein, 1999). In this way it is possible that the strict diet could result in impaired spatial reference memory only in females. 
Chapter 5: The effects of food restriction on rats' radial maze performance

There is some evidence that long-term dieting reduces cognitive performance in women, which may be relevant to my results (Green \& Rogers, 1995; Green et al., 1997). Attention span and working memory were reduced in a way that was directly proportional to self-rated "desire to eat". However, this effect was limited to spontaneously dieting women of normal weight, and was not apparent when diets were imposed on overweight women (Bryan \& Tiggemann, 2001). I have been unable to find any studies on the cognitive effects of dieting in men.

Other studies that have looked at sex differences in RAM performance do not display any obvious relationship between the level of food deprivation used and whether sex differences are found (table 5.2). However, none of these studies directly compared different levels of food restriction, and factors that may affect sex differences in spatial ability other than food restriction regime, may also have varied between the studies.

The female strict and moderate diet groups did not differ significantly in weight at the end of the experiment. This result was surprising as I expected the different diet regimes to result in the female groups having different weights. Female SpragueDawley rats do not gain weight very rapidly above 200g (B\&K Universal Group Catalogue), therefore the weight gains calculated from the suppliers' charts for the female groups were not very different for the two groups $(9.5 \mathrm{~g}$ per week for the moderate diet females compared to $8.5 \mathrm{~g}$ per week for the strict diet females). However, the moderate diet females were less likely than the strict diet females to finish their food allowance, i.e. they were more likely to be satiated at the start of the experiment. Therefore, although the female rats on the moderate diet did not have different weights to the female strict diet group, they were probably still differently motivated. It would have been useful to use an independent measure, such as progressive ratios schedules of reinforcement, to assess the level of food deprivation in the different groups. 
Table 5.2: The food restriction regimes used in studies of sex differences in laboratory rats' spatial ability. All comparisons were between control (unmanipulated) rats unless otherwise stated.

\begin{tabular}{|c|c|c|}
\hline Reference & Food restriction regime & $\begin{array}{l}\text { Sex differences in } \\
\text { spatial ability? }\end{array}$ \\
\hline $\begin{array}{l}\text { Boakes et al., } \\
2000\end{array}$ & Unrestricted food & No \\
\hline $\begin{array}{l}\text { Kolb \& Cioe, } \\
1996\end{array}$ & $18 \mathrm{~g}$ of food per day & No \\
\hline $\begin{array}{l}\text { Seymoure et al., } \\
1996\end{array}$ & $\begin{array}{l}85 \% \text { compared to unrestricted control } \\
\text { rats }\end{array}$ & Yes \\
\hline $\begin{array}{l}\text { Luine \& } \\
\text { Rodriguez, } 1994\end{array}$ & $\begin{array}{l}80-85 \% \text { normal body-weight with } \\
\text { growth allowance of } 5 \mathrm{~g} \text { per week }\end{array}$ & $\begin{array}{l}\text { Yes (gonadectomised } \\
\text { rats) }\end{array}$ \\
\hline Roof, 1993b & $85 \%$ normal body-weight & Yes \\
\hline $\begin{array}{l}\text { Williams et al., } \\
1990\end{array}$ & $85 \%$ free-feeding body-weight & $\begin{array}{l}\text { Yes (gonadectomised } \\
\text { rats) }\end{array}$ \\
\hline $\begin{array}{l}\text { Van Haaren et } \\
\text { al., } 1987\end{array}$ & 23hr food deprivation & No \\
\hline $\begin{array}{l}\text { Maier \& } \\
\text { Pohorecky, } \\
1986\end{array}$ & $\begin{array}{l}\text { Restricted food "sufficient to maintain } \\
\text { body-weight with moderate weight } \\
\text { gain" }\end{array}$ & No \\
\hline $\begin{array}{l}\text { Juraska et al., } \\
1984\end{array}$ & $\begin{array}{l}85 \% \text { compared to unrestricted control } \\
\text { rats }\end{array}$ & No \\
\hline Tees et al., 1981 & $\begin{array}{l}85 \% \text { pre-deprivation body-weight with } \\
\text { growth allowance of } 3 \mathrm{~g} \text { per week }\end{array}$ & Yes \\
\hline Einon, 1980 & Unrestricted food & Yes \\
\hline
\end{tabular}

Male rats showed more anxious behaviour than females in the black-white box, confirming previous data from my lab (Beveridge, 2001). I have been unable to find any other studies that directly compare male and female rats on the black-white box test of anxiety, but female rats are less anxious than males on other tests of anxiety such as the elevated plus maze (Johnston \& File, 1991; Fernandes et al., 1999). Males on the strict diet showed particularly anxious behaviour in the black-white 
box. Higher levels of food restriction may increase anxiety in male, but not female, rats, but further testing is necessary: the subjects should be tested in the black-white box before, as well as during, food restriction to be sure that any effect is not due to one group being randomly assigned more anxious individuals.

Within subjects on the moderate diet, females took less time per arm than males at the start of the experiment. In the experiments reported in chapters 2 and $4 \mathrm{I}$ did not find sex differences in the mean time that (unstressed) male and female rats spent in each arm, despite the fact that female rats have been shown to be more active than males using other measures (Barrett \& Ray, 1970; Dawson et al., 1973; Magalhaes \& Carlini, 1974; Dawson et al., 1975; Stewart et al., 1975; Krasnoff \& Weston, 1976). Male rats on stricter food restriction regimes have previously been shown to run faster to find food than subjects on less strict regimes (Zaretsky, 1966). This trend is apparent when comparing the activity of my male groups, but the effect of diet was in the opposite direction for the females. At present I do not have an explanation for this result.

Unlike in my previous two RAM experiments there were no significant sex differences in the number of uneaten reward errors made (although the trend was in the expected direction), nor were there sex differences in spatial ability when uneaten reward errors were included in the data. This could be due to subtle differences between the methods used in this experiment and those used in the earlier experiments. A possible example of this is the extra pretraining in this experiment (140min as opposed to $100 \mathrm{~min}$ and $85 \mathrm{~min}$ in the earlier experiments), which could have an effect because the frequency of uneaten reward errors decreases with training. There were no significant differences in the number of uneaten reward errors made between the strict and moderate diet groups. Therefore, the tendency to make uneaten reward errors does not seem to be related to the level of food motivation.

Within each of the groups, rats that gained more weight tended to make more reference memory errors. Although this effect is in the direction predicted (assuming that weight gain is inversely proportional to motivation), it is in the opposite 
direction to the only significant difference between the deprivation groups. This, combined with fact that it is not seen in the earlier experiments, suggests that this result may be an experimental artefact.

\section{Future work:}

1. The sex difference in the effects of food restriction level on reference memory could potentially explain some of the observed sex differences in spatial ability in laboratory rats. High levels of food restriction appear to reduce female reference memory, but do not affect that of male rats. This experiment should be replicated in order to see whether this somewhat surprising result is reproducible.

2. As the two female groups did not differ in weight despite being on different dietary regimes, it would be useful to compare groups of female rats that were on diets that resulted in different weights, and because the female moderate diet group only finished their food allowances on approximately half of the days it may also be useful to compare restricted and non-restricted females.

3. If high levels of food restriction are found to specifically decrease female spatial ability, I could test whether the effect is due to the level of food restriction affecting cognition, or motivation to find food in the maze. This could be done by manipulating the food motivation, such as by changing the palatability of the reward or the ease with which it is obtained; alternatively, the subjects could be kept on food restriction regimes but tested in the adversely motivated Morris water maze.

4. If reduced female spatial ability is found to be due to strict food restriction, further experiments could involve the manipulation of landmark cues. This would test whether the effect is mediated by reduced cue learning in rats on strict food restriction regimes as suggested by Telegdy and Cohen's (1971) study.

However, sex differences in the effects of different food restriction regimes cannot provide a complete explanation of the observed sex differences in spatial ability in rats, as sex differences have also been found on working memory RAM tasks and non-appetitively motivated tasks such as the water maze (table 1.2). 


\section{Chapter 6: Sex differences in spatial ability and hippocampal volume in Mongolian gerbils (Meriones unguiculatus)?}

\subsection{Introduction}

One of the best-supported hypotheses to explain the evolution of sex differences in spatial ability is the 'range size' hypothesis (Gray \& Buffery, 1971). This hypothesis states that spatial ability is directly proportional to range size and that there will only be sex differences in spatial ability when there are also sex differences in range size (section 1.2.5). There is a correlation between sex differences in spatial ability and range size for all of the species for which there are data available (table 6.1).

Table 6.1: The correlation between sex differences in range size and spatial ability.

\begin{tabular}{|c|c|c|c|}
\hline Species & $\begin{array}{l}\text { Sex differences } \\
\text { in range size? }\end{array}$ & $\begin{array}{l}\text { Sex differences in } \\
\text { spatial ability? }\end{array}$ & References \\
\hline $\begin{array}{l}\text { Meadow } \\
\text { vole }\end{array}$ & Male >female & Male>female & $\begin{array}{l}\text { Gaulin \& Fitzgerald, 1986; } \\
1989\end{array}$ \\
\hline Pine vole & No & No & Gaulin \& Fitzgerald, 1986 \\
\hline Prairie vole & No & No & Gaulin \& Fitzgerald, 1989 \\
\hline $\begin{array}{l}\text { Laboratory } \\
\text { mouse }\end{array}$ & Male>female & Male>female & $\begin{array}{l}\text { Chambers et al., 2000; } \\
\text { Mishima et al., } 1986\end{array}$ \\
\hline $\begin{array}{l}\text { Kangaroo } \\
\text { rat }\end{array}$ & No & No & Langley, 1994 \\
\hline Deer mouse & Male>female & Male>female & Galea et al., 1994a \\
\hline Human & Male>female & Male>female & Gaulin \& Hoffman, 1998 \\
\hline
\end{tabular}

The earliest test of the range size hypothesis was a comparison between two closely related vole species: promiscuous meadow voles (Microtus pennsylvanicus) and 
monogamous pine voles (M. pinetorum, Gaulin \& Fitzgerald, 1986). Using radio transmitters to measure the range sizes of the voles in the wild, Gaulin and Fitzgerald found that male meadow voles had larger ranges than conspecific females, but there were no differences in range size between male and female pine voles. They then captured individuals from their study site and tested them in the Tolman sunburst maze. The Tolman sunburst maze is a laboratory test of spatial ability where the subjects are trained to find food in a certain location in the maze, they are then tested to see if they can find an alternative route to the food location when the route that they have been trained to use is blocked. As predicted, male meadow voles performed better on the spatial task than female meadow voles, but there was no difference between the performance of the male and female pine voles.

A second study using monogamous prairie voles (M. ochrogaster) instead of pine voles, and symmetrical mazes (similar to the Davenport maze, figure 1.2.c) to test spatial ability, confirmed the earlier findings by showing that male meadow voles had larger ranges and better spatial ability than female meadow voles, while there were no sex differences in either range size or spatial ability in prairie voles (Gaulin \& Fitzgerald, 1989).

Wild male Mongolian gerbils (Meriones unguiculatus) have ranges that are approximately twice as large as those of conspecific females (Agren et al., 1989). Therefore the range size hypothesis predicts that male gerbils should perform better on spatial tasks than females.

The hippocampal region of the brain is associated with spatial ability (e.g. Morris et al., 1982; Sherry \& Vaccarino, 1989; Hampton \& Shettleworth, 1996; Strasser et al., 1998). Both between and within species larger hippocampi are associated with higher spatial demands (e.g. Rehkämper et al., 1988; Healy \& Krebs, 1992; Jacobs \& Spencer, 1994; Reboreda et al., 1996; table 6.2). Sherry et al. (1996) found that male gerbils have significantly larger hippocampal volumes relative to the rest of the telencephalon than females. This correlates with the observed sex difference in range size and adds to the prediction that male Mongolian gerbils will exhibit better spatial ability than conspecific females. 
Chapter 6: Sex differences in spatial ability and the hippocampus in gerbils?

Table 6.2: The intra-specific relationship between space-use and hippocampal volume. See text or reference for Latin names.

\begin{tabular}{|c|c|c|c|}
\hline Reference & Species & $\begin{array}{l}\text { Sex differences in } \\
\text { space-use? }\end{array}$ & $\begin{array}{l}\text { Sex differences in } \\
\text { relative hippocampal } \\
\text { volume? }\end{array}$ \\
\hline \multirow[t]{2}{*}{$\begin{array}{l}\text { Jacobs et al., } \\
1990\end{array}$} & Meadow vole & $\begin{array}{l}\text { Yes, males have } \\
\text { larger home-ranges }\end{array}$ & Yes, male>female \\
\hline & Pine vole & $\begin{array}{l}\text { No sex differences in } \\
\text { range size }\end{array}$ & No \\
\hline \multirow[t]{2}{*}{$\begin{array}{l}\text { Jacobs \& } \\
\text { Spencer, } 1994\end{array}$} & $\begin{array}{l}\text { Merriam's } \\
\text { kangaroo rat }\end{array}$ & $\begin{array}{l}\text { Yes, males have } \\
\text { larger home-ranges }\end{array}$ & Yes, male>female \\
\hline & $\begin{array}{l}\text { Bannertail } \\
\text { kangaroo rat }\end{array}$ & $\begin{array}{l}\text { Yes, males have } \\
\text { larger home-ranges }\end{array}$ & Yes, male>female \\
\hline $\begin{array}{l}\text { Sherry et al., } \\
1996\end{array}$ & $\begin{array}{l}\text { Mongolian } \\
\text { gerbil }\end{array}$ & $\begin{array}{l}\text { Yes, males have } \\
\text { larger range sizes }\end{array}$ & Yes, male>female \\
\hline \multirow[t]{3}{*}{$\begin{array}{l}\text { Sherry et al., } \\
1993\end{array}$} & $\begin{array}{l}\text { Brown-headed } \\
\text { cowbird }\end{array}$ & $\begin{array}{l}\text { Yes, only females } \\
\text { search for host nests }\end{array}$ & Yes, female>male \\
\hline & $\begin{array}{l}\text { Red-winged } \\
\text { blackbird }\end{array}$ & $\begin{array}{l}\text { No, species is not a } \\
\text { brood parasite }\end{array}$ & No \\
\hline & $\begin{array}{l}\text { Common } \\
\text { grackle }\end{array}$ & $\begin{array}{l}\text { No, species is not a } \\
\text { brood parasite }\end{array}$ & No \\
\hline \multirow[t]{3}{*}{$\begin{array}{l}\text { Reboreda et } \\
\text { al., } 1996\end{array}$} & Shiny cowbird & $\begin{array}{l}\text { Yes, only females } \\
\text { search for host nests }\end{array}$ & Yes, female>male \\
\hline & $\begin{array}{l}\text { Screaming } \\
\text { cowbird }\end{array}$ & $\begin{array}{l}\text { No, both sexes } \\
\text { search for host nests }\end{array}$ & No \\
\hline & $\begin{array}{l}\text { Bay-winged } \\
\text { cowbird }\end{array}$ & $\begin{array}{l}\text { No, species is not a } \\
\text { brood parasite }\end{array}$ & No \\
\hline $\begin{array}{l}\text { Petersen \& } \\
\text { Sherry, } 1996\end{array}$ & $\begin{array}{l}\text { Black-capped } \\
\text { chickadee }\end{array}$ & $\begin{array}{l}\text { No sex differences in } \\
\text { food-storing } \\
\text { behaviour }\end{array}$ & No \\
\hline
\end{tabular}

Studies in rats have directly linked the spatial ability of individual subjects to a parameter in their brains, namely the width of the dentate gyrus granule cell layer (DG-GCL) in the hippocampus. The DG-GCL is sexually dimorphic in rats, meadow voles in breeding condition, and mice, with that of males being wider than 
females (Roof \& Havens, 1992; Galea et al., 1999; Tabibnia et al., 1999). In rats there is a positive correlation between the width of the DG-GCL and spatial ability in the Morris water maze (MWM; Roof \& Havens, 1992; Roof, 1993a).

I predicted that male gerbils would have better spatial ability than female gerbils, because male gerbils have larger ranges and hippocampi than females. In order to test this I trained male and female gerbils on both the radial arm maze (RAM) and MWM. Gerbils are able to learn both of these tasks (e.g. Wilkie \& Slobin, 1983; Carney et al., 1991; Kondo et al., 1997; Zhou et al., 2001; Iqbal et al., 2002). Both RAM and MWM tasks were used because, although they both test spatial ability, they use different motivations and rewards and may give different results (Hodges, 1996). Although the MWM task is commonly used to test spatial ability in gerbils, this species is not a natural swimmer and the stress associated with the task may affect the results. By comparing the MWM with the RAM I intended to validate its results.

In order to use a suitably palatable food reward in the RAM I carried out a food preference test prior to testing in the RAM. All behavioural experiments were carried out between February-May 2002. After testing the gerbils were sacrificed and their brains removed, sectioned and mounted onto slides. This was an attempt to replicate Sherry et al.'s (1996) finding, and to make a direct comparison between DG-GCL width and spatial ability within individual gerbils.

\subsection{Food preference test}

\subsubsection{Materials and methods}

Subjects were 10 female and 10 male gerbils, locally bred and approximately 3 months old. The males weighed between $65 \mathrm{~g}$ and $90 \mathrm{~g}$ (mean $76 \mathrm{~g}$ ), and the females weighed between $55 \mathrm{~g}$ and $100 \mathrm{~g}$ (mean $74 \mathrm{~g}$ ) when they arrived in the animal house at the end of January 2002. They were housed in same-sex groups of five in the same cages and animal house conditions described in section 2.2.2. In addition to a floor covering of wood-chips their cages also contained shredded tissue paper and a piece of cardboard (toilet paper tube or half an egg box), which the gerbils shredded to 
make nesting material. The cage bases were replaced once a week and the gerbils were given fresh supplies of wood-chips, tissue paper and cardboard with the new base. They had ad libitum access to water and food (RM3 irradiated diet from Special Diet Services) in their home cages. Each subject was identifiable by coloured ink markings on their tails, which were reapplied every 3-4 days.

The food rewards used were shelled sunflower seeds (Helianthus annuus), half pinenuts (Pinus pinea) and Sugar Puffs breakfast cereal (Quaker). These potential food rewards were chosen because they were thought likely to be especially palatable to gerbils.

As gerbils can be prone to seizures brought on by the stress of being handled or put in novel environments (Norris, 1987), all subjects were handled daily for a couple of minutes for seven days prior to the food preference test in order to reduce stress. After handling, 10 pieces of each of the food types were placed in each cage. Two of the metal eggcups used in the test were left in each cage for four days. This was an attempt to reduce stress by allowing the subjects to become familiar with some of the novel aspects of the test before testing.

Due to a mistake in sexing the gerbils before they arrived in the animal house a male was housed with the females. This male was excluded from the experiment, as were two females that gave birth. It was unknown whether any, or all, of the remaining females were pregnant. Seven females and 10 males were used in the food preference tests.

For the food preference test a clean standard sized cage was used. It had wood-chips on the floor but no water bottle or food pellets. Three metal eggcups were placed at equal intervals along the short side of the cage at the opposite end to the food hopper (figure 6.1). Each eggcup contained 10 pieces of one of the three foods. Each cup's content was varied in the order sugar puffs:sunflower seeds:pine-nuts between trials in order to control for possible location biases.

The test was carried out on $12 / 2 / 02$ between 1330 and 1600 in a laboratory within the animal house. Subjects were given the test in a pseudorandom order so that roughly 
Chapter 6: Sex differences in spatial ability and the hippocampus in gerbils?

equal numbers of each sex were tested in each half of the experiment (it was not possible to use exactly equal numbers of each sex because more males were tested than females). Each subject was placed in the testing cage on its own and the number of pieces of each type of food eaten were recorded, after $5 \mathrm{~min}$ had elapsed the subject was returned to its home cage. The eggcups were replaced if they had been moved and were refilled before the next subject was put in the cage.

Figure 6.1: Elevated view of the set-up used for the food preference test. Not to scale.

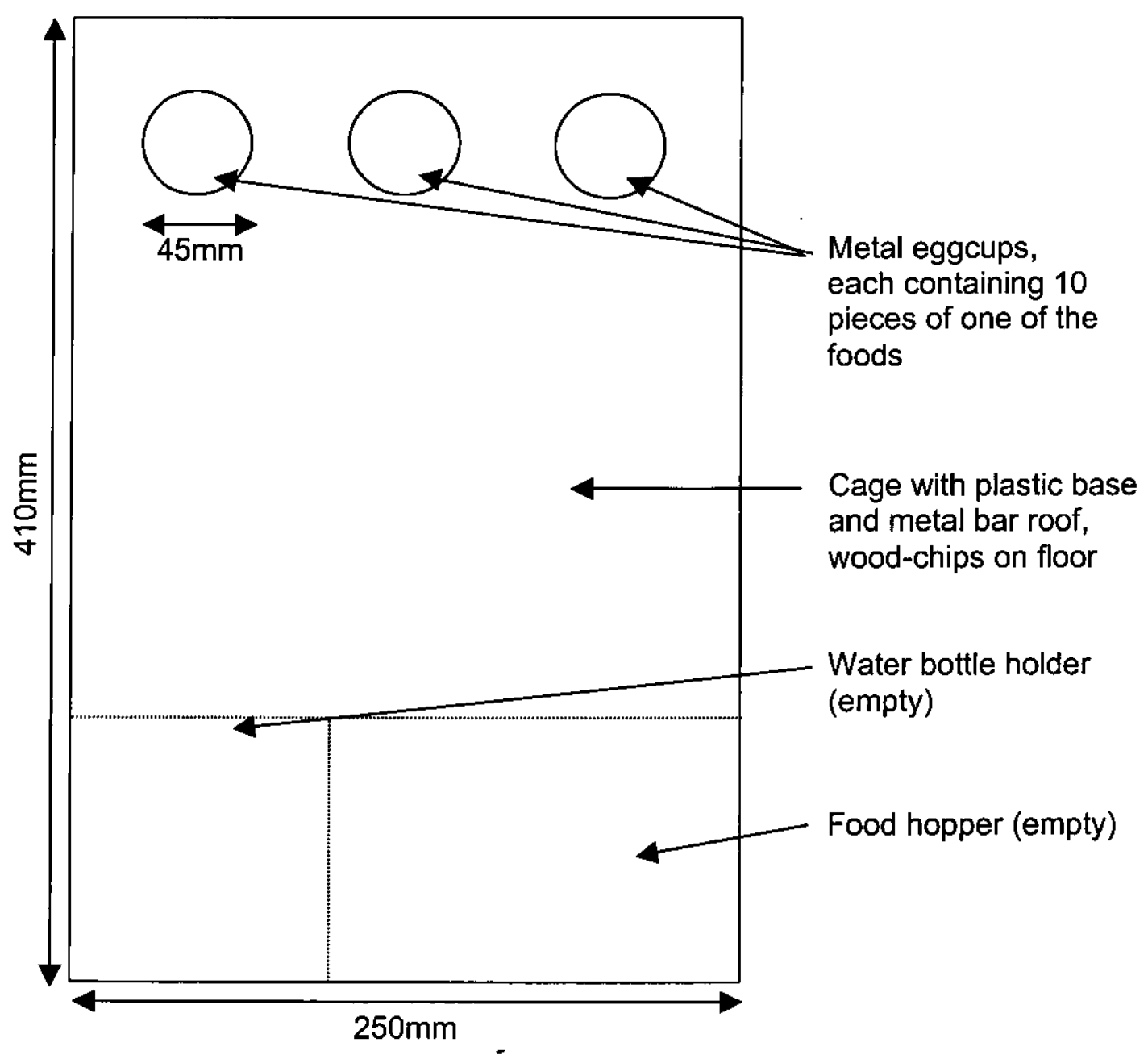




\subsubsection{Results and analysis}

The number of pieces eaten of each of the food types was analysed in Minitab using the following general linear model (GLM):

Number of pieces eaten $=\operatorname{sex}+$ gerbil $(\operatorname{sex})+$ food type + food type*sex

This model was equivalent to a repeated-measures analysis of variance (ANOVA) with one between-subjects variable (sex) and one within-subject variable (food type). The F-ratio for sex was calculated using the 'gerbil(sex)' mean square (MS) as the denominator. The interaction term was non-significant and so was removed from the analysis. There was no significant effect of sex on the number of pieces of food eaten $\left(\mathrm{F}_{1,15}=0.37, \mathrm{p}=0.552\right)$, but food type had a significant effect on the number of pieces eaten $\left(\mathrm{F}_{2,32}=13.98, \mathrm{p}<0.001\right)$. Tukey simultaneous tests showed that more pine-nut halves were eaten than either sunflower seeds or Sugar Puffs (pine-nuts versus Sugar Puffs $\mathrm{T}=-5.285, \mathrm{p}<0.0001$; versus sunflower seeds $\mathrm{T}=-2.779$, $\mathrm{p}=0.0239)$. More sunflower seeds were eaten than Sugar Puffs $(T=-2.506$, $\mathrm{p}=0.0449$ ). Pine-nuts were the preferred food type (figure 6.2).

Figure 6.2: Results of the food preference test. Male $n=10$, female $n=7,+/-$ standard error of the mean (SEM).

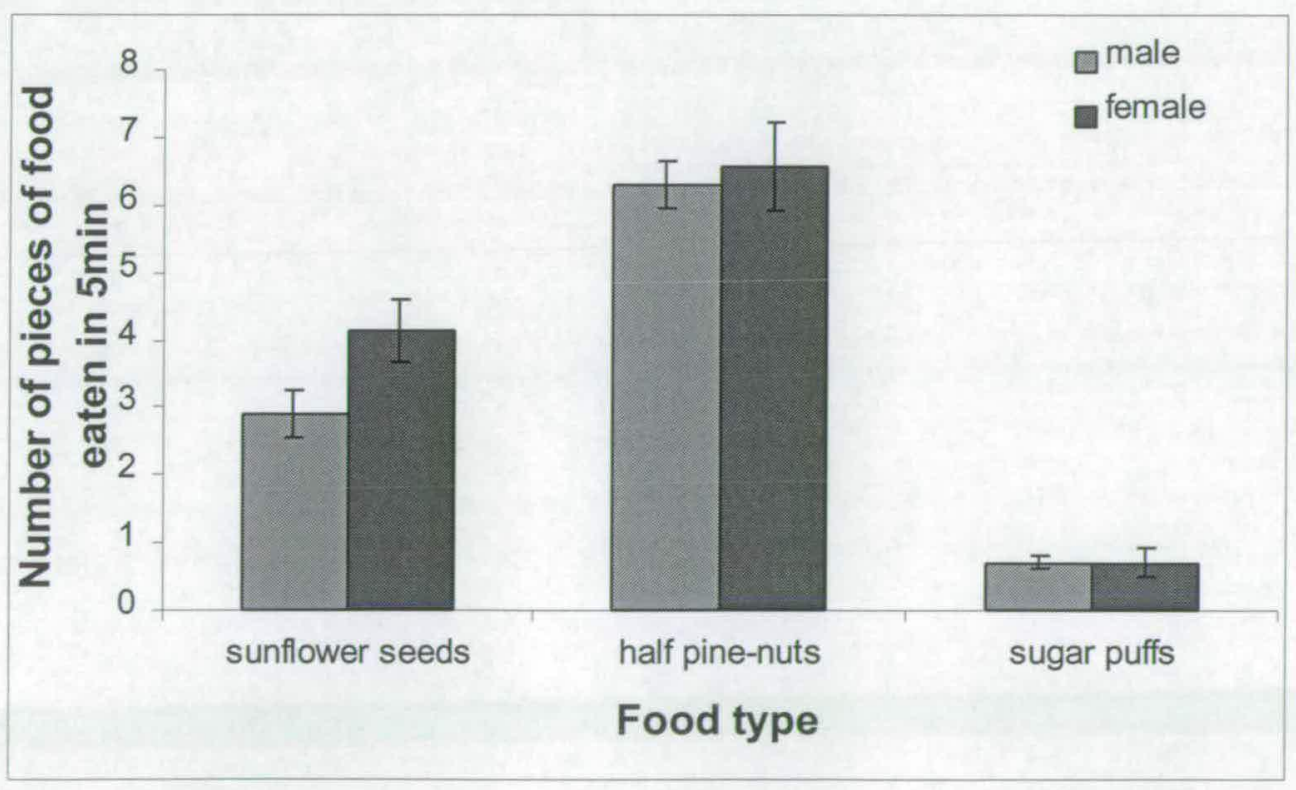




\subsubsection{Discussion}

As pine-nuts were the most preferred food these were chosen as food rewards for the RAM task. There were no sex differences in the overall number of food pieces eaten during the food preference test, nor was one food preferred more by one sex than the other. This suggests that both sexes preferred pine-nuts equally and were equally motivated to eat. Therefore, there were no sex differences in motivation that could potentially cause sex differences in spatial ability in the RAM (chapter 5).

It is possible that these results may have been affected by the fact that some of the female gerbils may have been pregnant, as pregnancy can affect both motivation to eat and food preferences in humans (e.g. Durnin, 1991; Pope et al., 1992). However, the gerbils had had ad libitum access to a nutritionally complete diet immediately prior to the food preference test, meaning that subjects of both sexes should not have been either food or nutrient deprived. Therefore, it was assumed that any effects of pregnancy were minimal.

It was not known whether any of the females were pregnant, and if so what trimester they were in. As spatial ability is reduced during the third trimester of pregnancy (Woodfield, 1984; Galea et al., 2000) new gerbils were obtained for the spatial testing. In order to ensure that the gerbils were not pregnant they were bought from a supplier outside the university. Due to time constraints the new gerbils were not given the food preference test, and it was assumed that the food preferences found in this test could be generalised to the new gerbils.

\subsection{Radial arm maze task}

\subsubsection{Materials and methods}

Subjects were eight male and eight female gerbils, bought from B\&K Universal Ltd. They were 12 weeks old when they arrived in the animal house on $14 / 2 / 02$. The males weighed between $60 \mathrm{~g}$ and $71 \mathrm{~g}$ (mean $64 \mathrm{~g}$ ) and the females weighed between $50 \mathrm{~g}$ and $69 \mathrm{~g}$ (mean 59g). They were housed in same-sex groups of four under the conditions described in section 6.2.1. Subjects were individually identifiable by 
coloured ink on their tails that was reapplied every 3-4 days. Each subject was weighed weekly during the experiment.

The gerbils were habituated to some of the features of the maze procedure before training began in order to reduce the stress associated with the maze training. Starting four days after arrival in the animal house all subjects were handled daily for seven days prior to being put in the RAM. After handling, 32 pine-nut halves were placed in each cage and two empty metal eggcups (used as food cups in the maze) were left in each cage for six days during the handling. For two days immediately before pretraining started the gerbils were taken on a trolley to the maze room, which was some distance from the animal house. They were left in the maze room in their home cages for $30 \mathrm{~min}$ before being returned to the animal house.

The eight-arm RAM was the same as that described in chapter 2, but it was housed in a different room. The new maze room measured approximately $3 \mathrm{~m}$ by $5 \mathrm{~m}$ and contained the water-maze already described in chapter 2. The RAM was placed on a platform inside the water-maze as previously described. The room also contained a number of visual and auditory cues, including two fluorescent lighting strips, a blacked-out window, a still, a radio continuously broadcasting music, shelving, and brightly-coloured posters (figure 6.3). Simple shapes such as a square and triangle, measuring approximately $200 \mathrm{~mm}$ by $200 \mathrm{~mm}$, were cut from coloured card and attached to the inside of the water-maze to provide additional visual cues because the sides of the water maze might obscure the gerbils' view of the landmarks in the room. It was hoped that by adding these additional landmarks the gerbils would be able to learn the maze faster and more accurately, because they would have more cues available to use (i.e. both local and distant cues). Studies in rats have suggested that females pay more attention to local cues than males (e.g. Williams et al., 1990; Roof \& Stein, 1999). Therefore, including local cues may have made sex differences in spatial ability more difficult to detect. However, I decided to include local cues for several reasons: first, it would potentially speed up training; second, using both cue types would be the best approximation of the conditions likely to be available in the gerbils' natural habitat; third, there is no evidence for sex differences in cue use 
Chapter 6: Sex differences in spatial ability and the hippocampus in gerbils?

in meadow voles, another species in which the range size hypothesis clearly predicts sex differences in spatial ability (section 1.2.5).

Figure 6.3: Diagram from above showing some of the features of the room in which the radial arm and Morris water maze experiments were carried out. Not to scale.

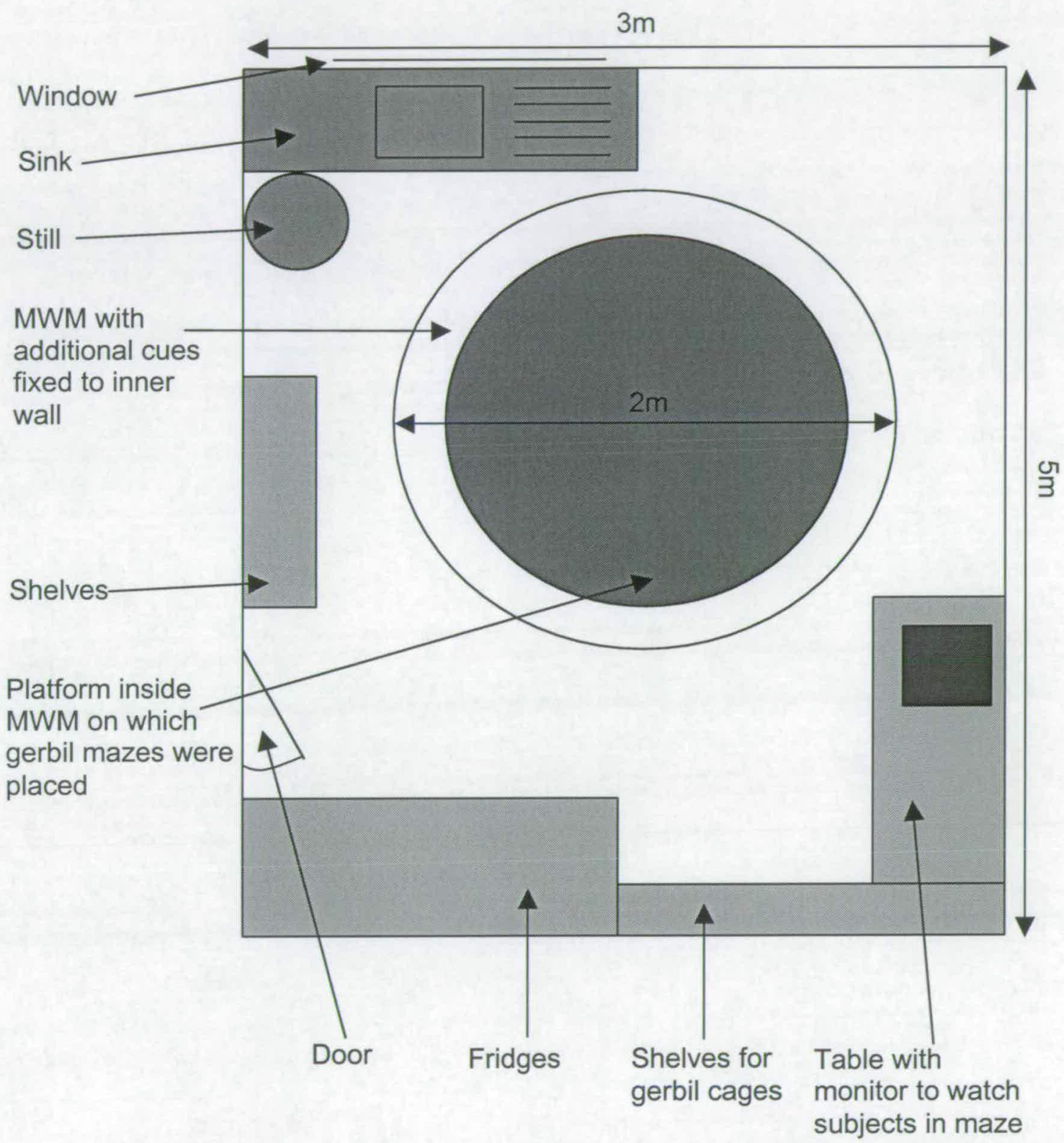




\section{Pretraining}

Pretraining in the RAM took six days and was designed to introduce the subjects to the apparatus with minimum stress.

Days $1 \& 2$ : subjects were put in the maze in groups of cage-mates for $15 \mathrm{~min}$ along with their daily ration of pine-nuts ( 32 halves). After $15 \mathrm{~min}$ the gerbils and remaining pine-nuts were put back in the home cage.

Day 3: two sessions (am \& pm). Subjects were put in the maze individually for $5 \mathrm{~min}$ in a pseudorandom order so that equal numbers of each sex were tested in each quarter of the experimental order. Eight pine-nut halves were put in the centre of the maze before each subject was placed in it. Each subject was then left in the maze for $5 \mathrm{~min}$ before being returned to its home cage.

Days 4, 5 \& 6: the release chamber and mild food deprivation were introduced. All of the food was removed from the cage hoppers upon arrival in the maze room. The gerbils were then left for $30 \mathrm{~min}$ before the first trial started. Before each trial eight pine-nut halves were put in the centre of the maze. Each subject was placed in the release chamber for $30 \mathrm{sec}$. The release chamber was then raised to start the trial, which lasted for $5 \mathrm{~min}$. Two sessions (am \& pm) were carried out each day. There was one rest day between days 4 and 5 and only the morning session was given on day 4.

\section{Working memory task}

Training started on the working memory RAM task on the day immediately following pretraining. Each subject was given two trials a day (am \& pm), for nine continuous days. The subjects were placed in the maze in a pseudorandom order, as described above. The morning trials started within $30 \mathrm{~min}$ of 1000 and the afternoon trials within 30min of 1400 . Upon arrival in the maze room the food was removed from the cage hoppers and the gerbils were left for $30 \mathrm{~min}$ before the trials started. The food was replaced in the hoppers before the gerbils were returned to the animal house at the end of the day. Prior to each trial half a pine-nut was placed in each of 
the eight food cups at the end of each maze arm. Each subject was placed in the release chamber for $30 \mathrm{sec}$ before it was raised and the trial started. The experimenter sat with her back to the maze and watched the subject on a monitor. The arm choices made by the subject were recorded, as was whether the reward was eaten. An arm choice was defined as an entry of more than $100 \mathrm{~mm}$ down an arm. The trial continued until all eight rewards were eaten, or for $10 \mathrm{~min}$. Each trial was recorded on videotape. The maze was wiped after every session and the arms relocated in a random order, the food cups were also washed and dried.

Two male gerbils developed the habit of escaping from the release chamber. When this happened the release chamber was raised and the trial was timed from when the gerbil climbed out. Occasionally gerbils escaped from the maze onto the platform supporting it. When this happened they were quickly replaced back into the arm from which they had escaped and the trial continued. On 24 trials out of 288 subjects climbed out of the maze and dropped into the empty water-maze below the RAM. When this happened the trial was stopped because of the time needed to recapture the subject. The data from these trials were included in the analysis, as the gerbils always entered all eight arms before escaping.

\section{Reference memory task}

Training started on the reference memory task three days after the working memory task training ended. Subjects were given two trials a day for 15 consecutive days. The method was the same as that used for the working memory task except that only four of the arms were rewarded. Each subject was assigned a different pattern of four rewarded arms that remained constant throughout the training. The patterns were chosen so that each one had exactly two adjacent arms so as to discourage subjects from forming response chains such as entering every arm, or entering every alternate arm, in order to solve the maze. The maximum time spent in the maze was 5 min as it was noted during the working memory task that if a subject was going to eat the rewards it did so within the first $5 \mathrm{~min}$. This also reduced the incidence of maze escapes. 


\subsubsection{Results and analysis}

There were eight missing data-points for the working memory task and six for the reference memory task, due to subjects not entering all of the rewarded arms within the allotted time. When data were missing the data-point was calculated by taking the mean of the previous and next trials. When the missing data-point occurred on the first or last trial the mean of the next two, or the previous two, trials was taken.

Data were analysed using Minitab. When parametric tests were used, the residuals and fitted values were checked to make sure that they met the assumptions of normality of error and homogeneity of residuals. Transformations were applied as appropriate. When general linear models were used full models were initially applied to the data, but any non-significant interaction terms were removed from the final analysis. The following GLM was used for repeated-measures tests:

Response $=\operatorname{sex}+$ gerbil $(\operatorname{sex})+$ day $+\operatorname{sex} *$ day

Sex was the between-subjects factor and day was the within-subject factor. The Fratio for the effect of sex was calculated by dividing 'sex' MS by 'gerbil(sex)' MS so that the appropriate degrees of freedom were used.

\section{Working memory task}

The mean of every two trials was calculated, so that the data were blocked by day. This removed effects of time of day on the trials; for example the subjects may have performed better in the afternoon because they were hungrier. The following response variables were analysed using the above GLM.

\section{1) Number of rewards eaten within $10 \mathrm{~min}$}

The number of pine-nut halves eaten during the $10 \mathrm{~min}$ trials was analysed for an effect of sex and day. The maximum number that could be eaten was eight. As the residuals from the repeated-measures ANOVA were non-normal, the GLM model 'number of rewards eaten $=$ sex' was applied to the mean number of rewards eaten for each individual over the 18 trials. There was not a statistically significant 
difference between the number of rewards eaten by the males and females $\left(F_{1,14}=0.65, p=0.435\right.$; figure 6.4.a). A directional Wilcoxon signed rank test was used to test whether the number of rewards eaten on day nine minus the number of rewards eaten on day one was greater than zero. The gerbils did not eat significantly more rewards on the last day of the experiment than on the first ( $\mathrm{N}$ used for test $=11$, Wilcoxon statistic $=36.5, \mathrm{p}=0.395$; figure 6.4.a).

In this way the gerbils' behaviour is unlike that of the rats. Although the rats sometimes went down an arm and failed to eat the reward, they never left rewards uneaten after $10 \mathrm{~min}$ in the maze and the incidence of these errors decreased rapidly with time (chapters $2,4 \& 5$ ). The gerbils did not seem to be highly motivated to eat the rewards (figure 6.4.a). There was a lot of variation among individuals (some subjects never ate a reward while others always ate all eight), but the average number of rewards eaten during a trial was 4.9 for females and 3.7 for males.

\section{2) Time per arm}

The mean time that each subject spent in each arm of the maze was calculated for each trial by dividing the total number of arms that the gerbil entered by the length of time that it was in the maze to give an estimate of activity in the maze. This measure assumed that the time spent in each arm did not change during the trial. The data were $\log 10$ transformed. The sex by day interaction was significant $\left(\mathrm{F}_{8,112}=2.79\right.$, $\mathrm{p}=0.007$ ). Tukey simultaneous tests showed that this was due to the females spending less time per arm than the males on days five and six (day $5 \mathrm{~T}=3.911$, $\mathrm{p}=0.0174$; day $6 \mathrm{~T}=3.977, \mathrm{p}=0.0140$; figure 6.4.b).

\section{3) Patrolling behaviour}

As the gerbils did not consistently eat the rewards, it did not seem appropriate to use any reward-motivated measures of working memory. Instead an explorationmotivated measure of working memory was used. This assumed that the subject was motivated to visit previously unvisited arms irrespective of food rewards. The number of novel arms entered during the first eight arm visits was calculated for each subject for each trial. 
If the subjects were choosing arms at random they would be expected to visit a mean of 5.25 rewarded arms during the first eight choices (sampling with replacement, Tillé et al., 1996). On average the subjects visited 7.0 novel arms in the first eight entries. A one-sample t-test was used to test whether the mean number of novel arms entered during the first eight entries was different from random. There was a significant difference $(\mathrm{n}=16, \mathrm{t}=22.97, \mathrm{p}<0.0001)$. The subjects entered more novel arms than would be expected if they were choosing arms at random.

I analysed the data using the repeated-measures GLM model. The sex by day interaction was not significant and was removed from the model. There was no difference between the sexes' performances $\left(F_{1,14}=1.02, p=0.330\right)$. The effect of day approached significance $\left(\mathrm{F}_{8,120}=1.73, \mathrm{p}=0.098\right)$. This seemed to be due to day-to-day variation, rather than any overall trend (figure 6.4.c). A paired t-test showed that there was no difference between the number of novel arm entries made on days one and nine $(\mathrm{T}=-0.70, \mathrm{p}=0.495)$.

Figure 6.4: Parameters measured during the working memory radial arm maze task, grouped by day and sex. Male $n=8$, female $n=8$. Each day is the mean of two 10 min trials. +/-SEM.

a) Mean number of rewards eaten.

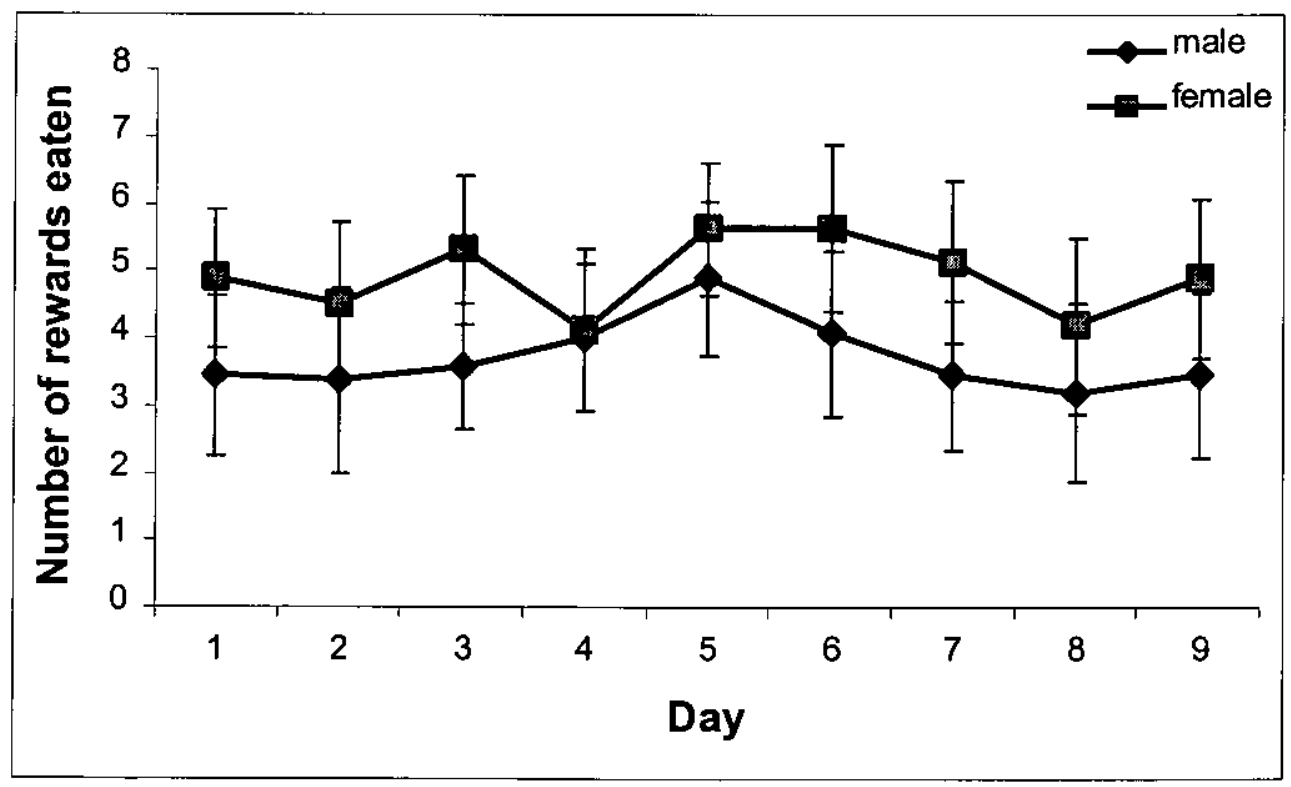


Chapter 6: Sex differences in spatial ability and the hippocampus in gerbils?

b) Average time spent in each arm.

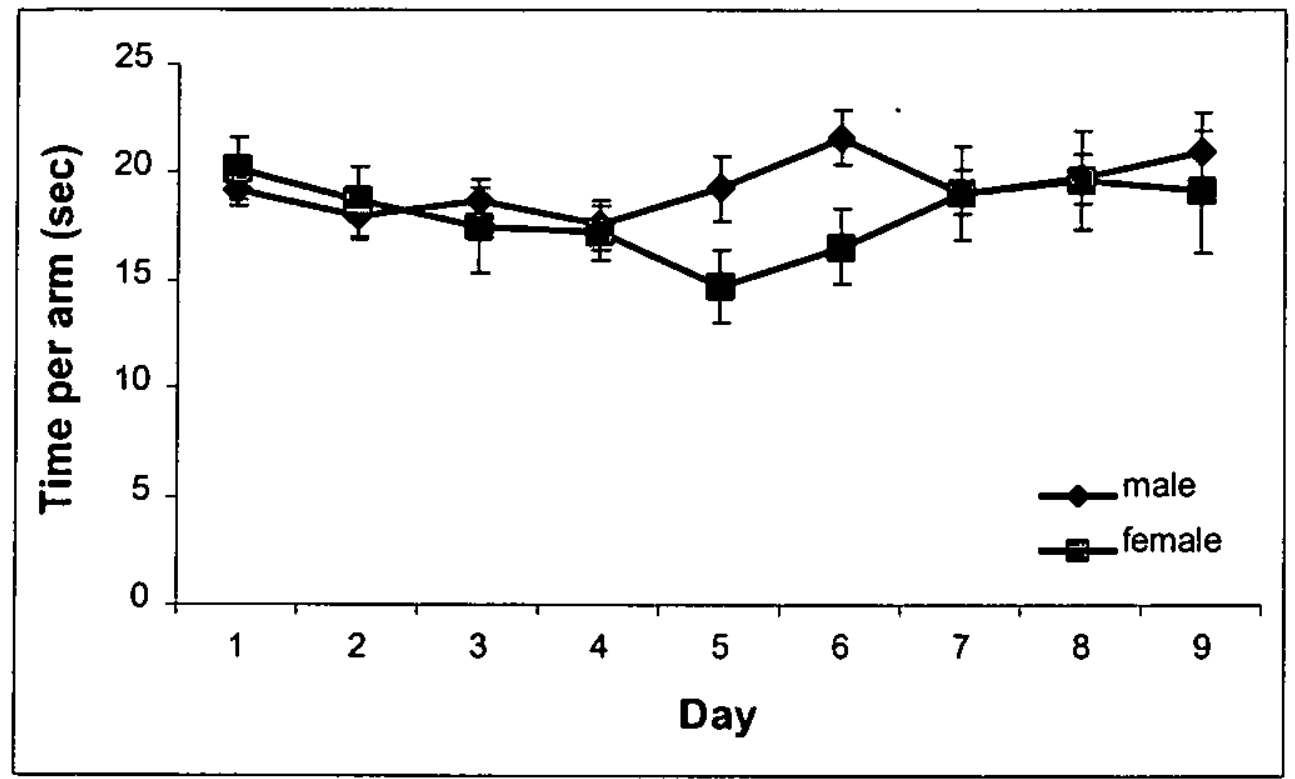

c) Number of novel arm entries made in the first eight arm entries.

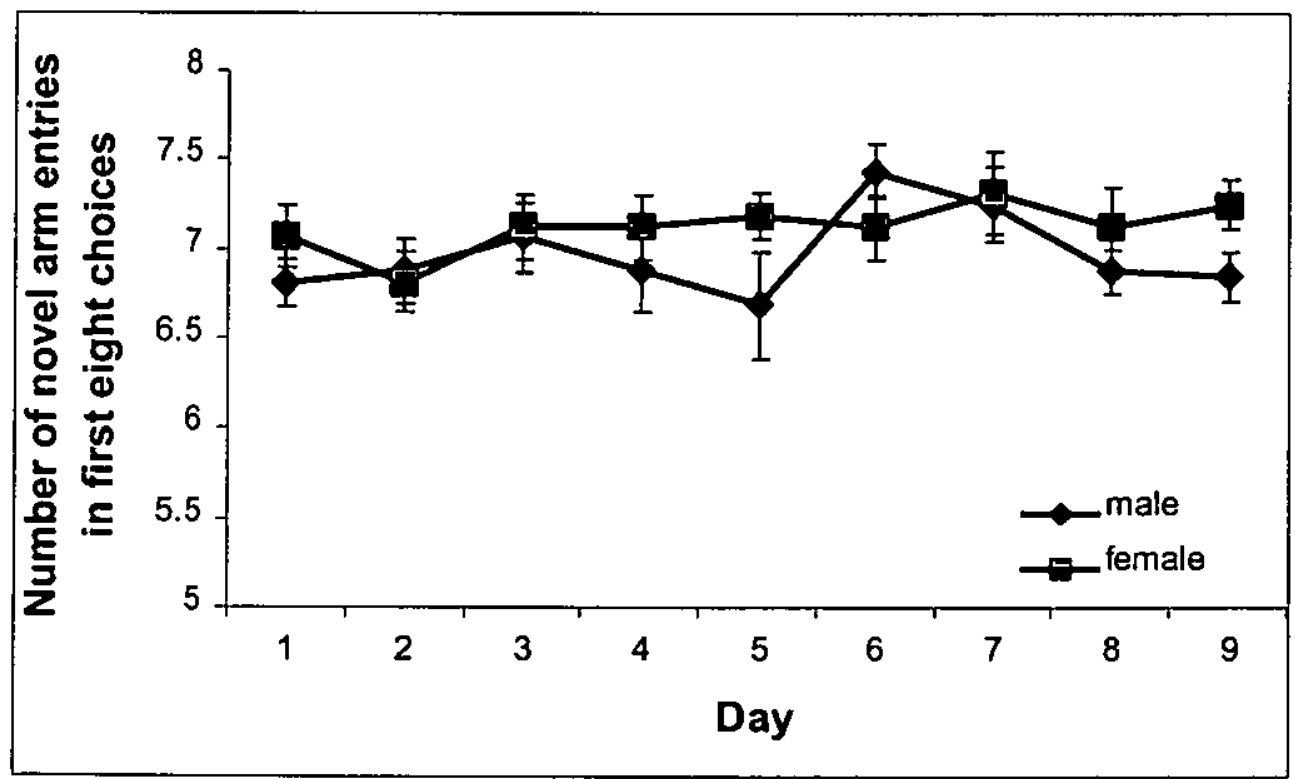




\section{Reference memory task}

Four of the subjects (three males and one female) were excluded from reference memory training because they consistently failed to eat the rewards during the working memory task. All of the reported data are from the remaining seven females and five males. The repeated-measures data were blocked by day (the mean of the am and pm trials).

Unlike in the working memory task, all of the rewards were eaten on most trials (301 out of 360; figure 6.5.a). As the number of rewards eaten data did not fulfil the parametric assumptions a non-parametric Mann-Whitney test was used to test for sex differences in the mean number of rewards that each individual ate per trial, averaged over all 30 trials. There were no differences between the sexes $(\mathrm{W}=45.0, \mathrm{p}=1.0000$ adjusted for ties). A directional Wilcoxon signed rank test was used to test whether the number of rewards eaten on day 15 minus the number of rewards eaten on day one was greater than zero. The effect was close to significance, the gerbils tended to eat more rewards on the last day of the experiment than on the first ( $\mathrm{N}$ used for test $=4$, Wilcoxon statistic $=10, \mathrm{p}=0.050$; figure 6.5.a).

The number of reference and working memory errors made were analysed using a repeated-measures ANOVA with one within-subject effect (day) and one betweensubjects effect (sex). Working memory errors (visits to an arm previously visited on a trial) and reference memory errors (visits to an arm that never contained food) were calculated for each subject on each trial. An entry into a rewarded arm was counted as 'correct' even if the reward was not eaten (i.e. this analysis was equivalent to the analyses excluding uneaten reward errors in chapter 2, $4 \& 5$ ). For the reference memory errors this method assumes that the gerbils were motivated by, and paying attention to, the pine-nuts even though they often did not eat them.

The working memory data were square-root transformed. The sex by day interaction was not significant for either measure and was removed from both analyses. The effect of day was significant for both working and reference memory errors (working $F_{14,154}=1.91, p=0.029$; reference $F_{14,154}=2.26, p=0.008$ ). The number of reference 
memory errors made decreased as the training progressed (figure 6.5.b). The number of working memory errors made was very variable throughout the experiment (figure 6.5.c). There was no effect of sex on the number of reference memory errors $\left(F_{1,10}=0.09, p=0.779\right)$. The effect of sex on the number of working memory errors made approached significance $\left(F_{1,10}=3.92, p=0.076\right)$. There was a tendency for males to make more working memory errors than females (figure 6.5.c).

The numbers of novel entries into a rewarded arm made during the first four arm choices were also analysed using the repeated-measures GLM. The sex by day interaction was non-significant. The performance of the sexes did not differ $\left(F_{1,10}=0.25, p=0.625\right)$, but there was a significant effect of day $\left(F_{14,154}=2.12\right.$, $\mathrm{p}=0.013$ ). This seems to be due to performance improving (i.e. more novel entries being made) as the experiment progressed (figure 6.5.d). If the subjects entered arms at random, but avoided previously visited arms (sampling without replacement) they would be expected to visit on average 2.0 novel rewarded arms during the first four choices (Tillé et al., 1996). This provided a conservative test of a departure from random behaviour, because the subjects' did sometimes visit previously visited arms (i.e. make working memory errors). The gerbils had already been shown to perform better than sampling with replacement in the working memory task analysis. As the number of novel rewarded arm entries increased as the experiment progressed, the averages of the two trials on day 1 , and the two trials on day 15 were tested to see if they differed from random. One-sample t-tests showed that the subjects did not perform differently to random on day 1 when they entered on average 2.3 novel rewarded arms $(\mathrm{n}=12, \mathrm{~T}=1.68, \mathrm{p}=0.1200)$, but they did perform better than random on day 15 when they entered an average of 2.7 novel rewarded arms $(n=12, T=3.96$, $\mathrm{p}=0.0022$ ).

A directional, paired t-test was used to test whether the gerbils made fewer reference memory errors on the last day of training than on the first. The gerbils did make significantly fewer reference memory errors on day 15 than on day $1(n=12, T=2.12$, $\mathrm{p}=0.030$ ). 
Figure 6.5: Parameters derived from the radial arm maze reference memory task, by day and sex. Male $n=5$, female $n=7$. Each day is the mean of two trials. $+/$-SEM.

a) Average number of rewards eaten.

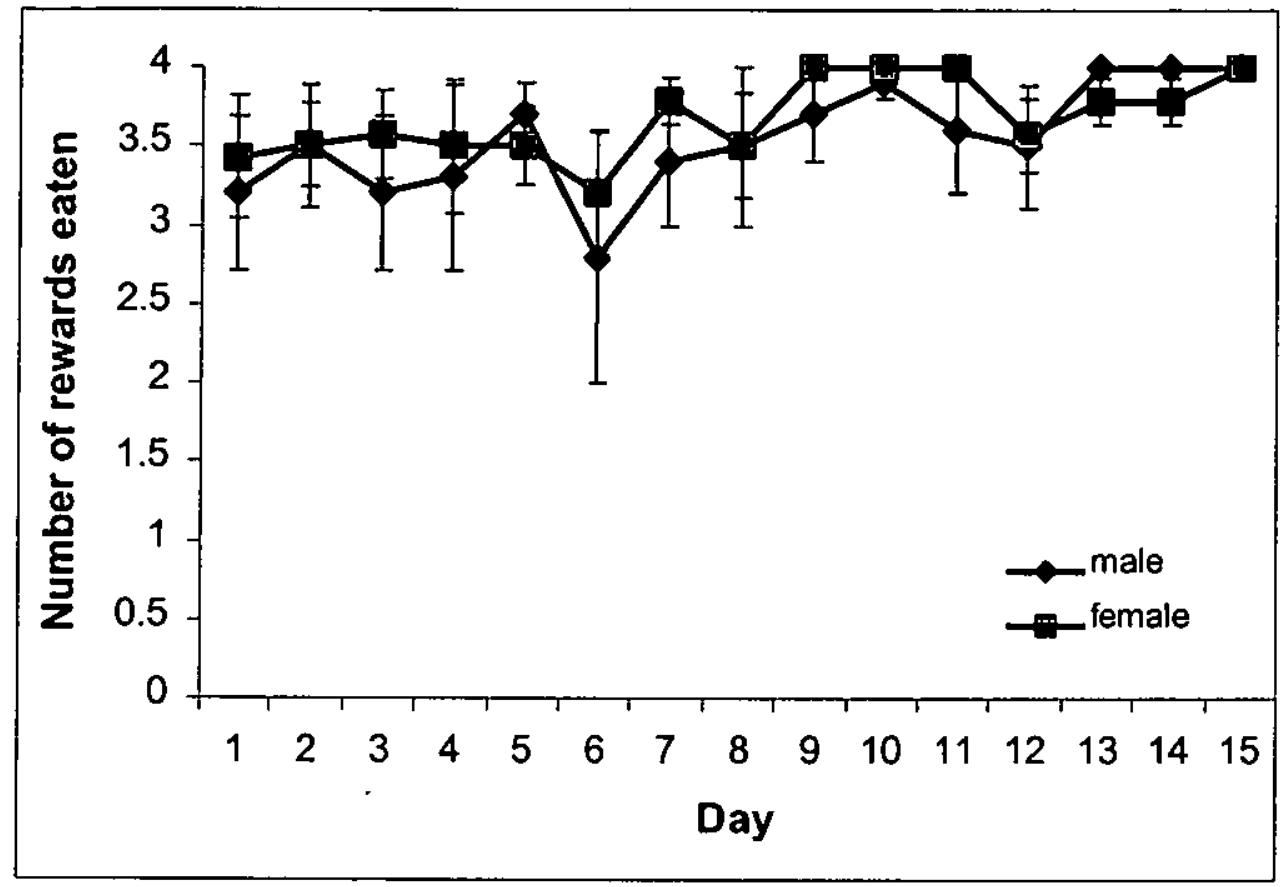

b) Reference memory errors (visits to an arm that never contains a food reward).

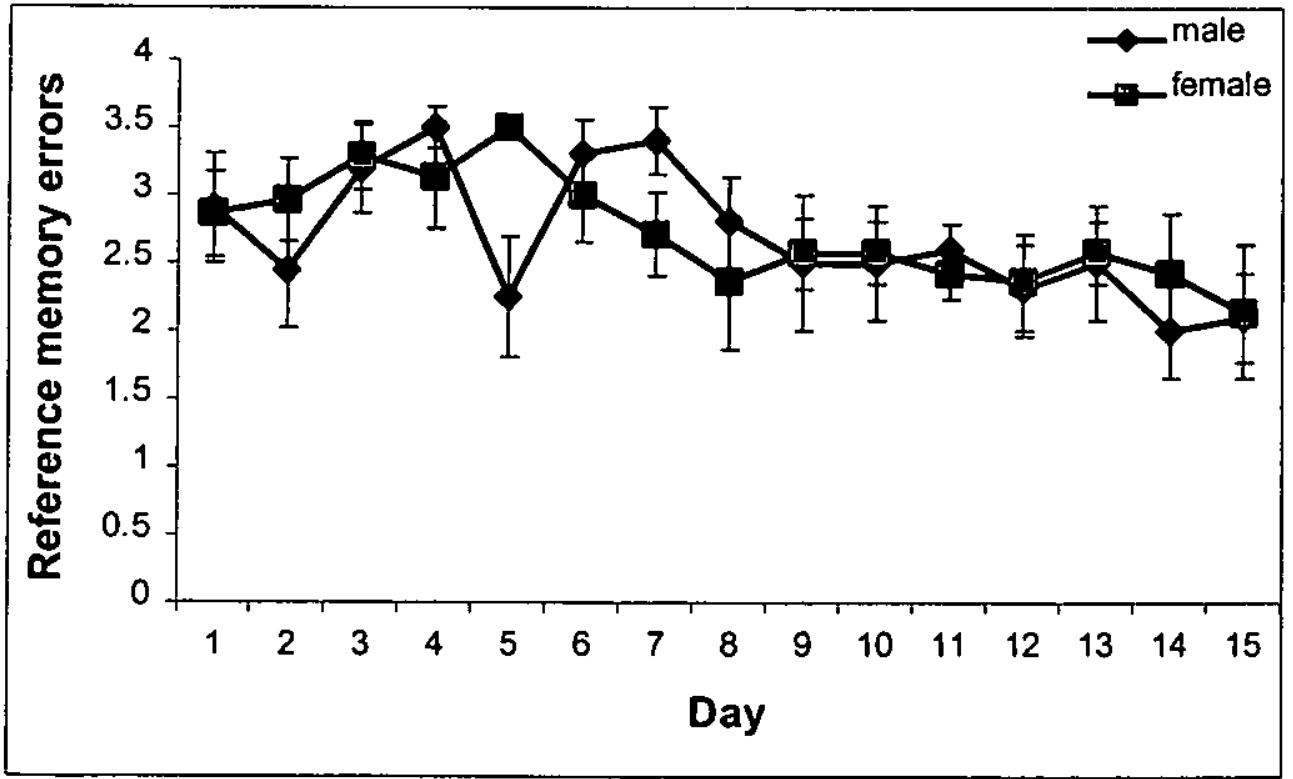


Chapter 6: Sex differences in spatial ability and the hippocampus in gerbils?

c) Working memory errors (visits to an arm previously visited on that trial).

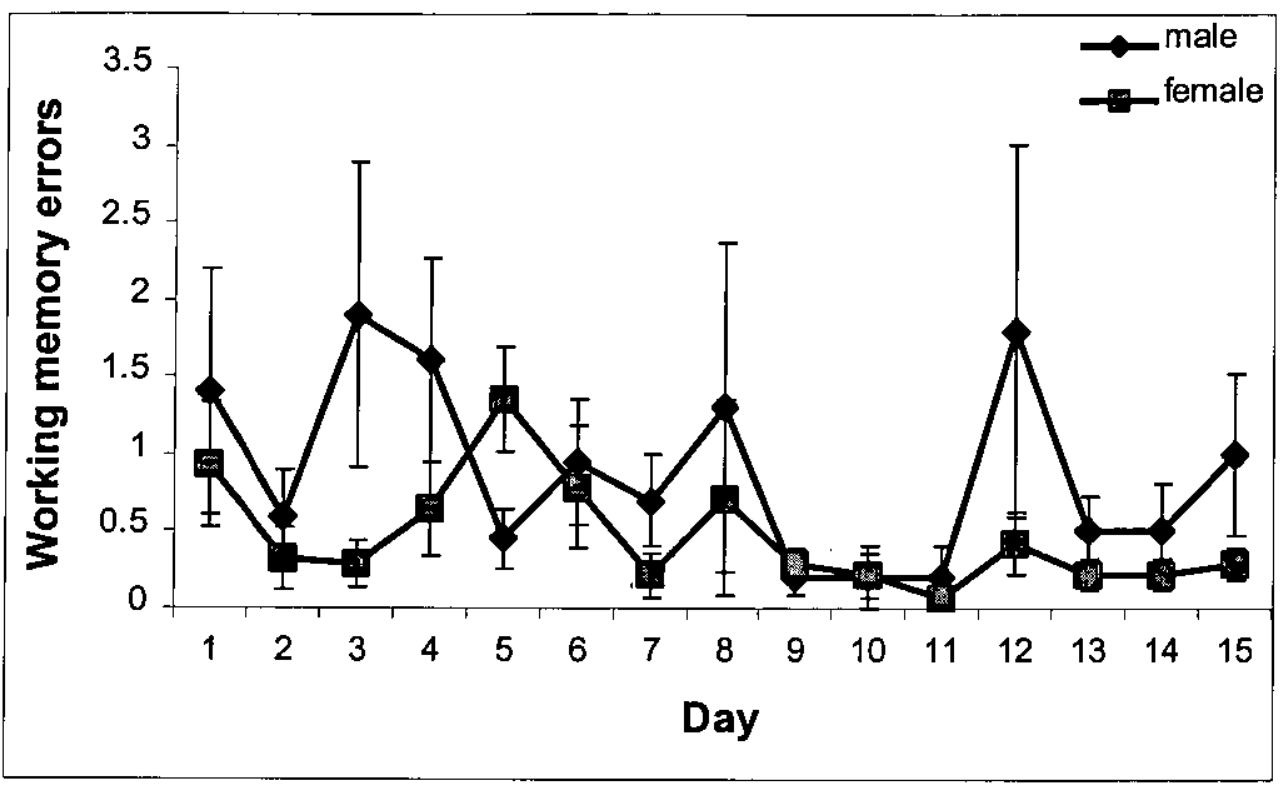

d) Number of novel entries to a rewarded arm out of the first four arm choices. Dashed line represents average random performance if subjects are sampling without replacement (i.e. do not make working memory errors).

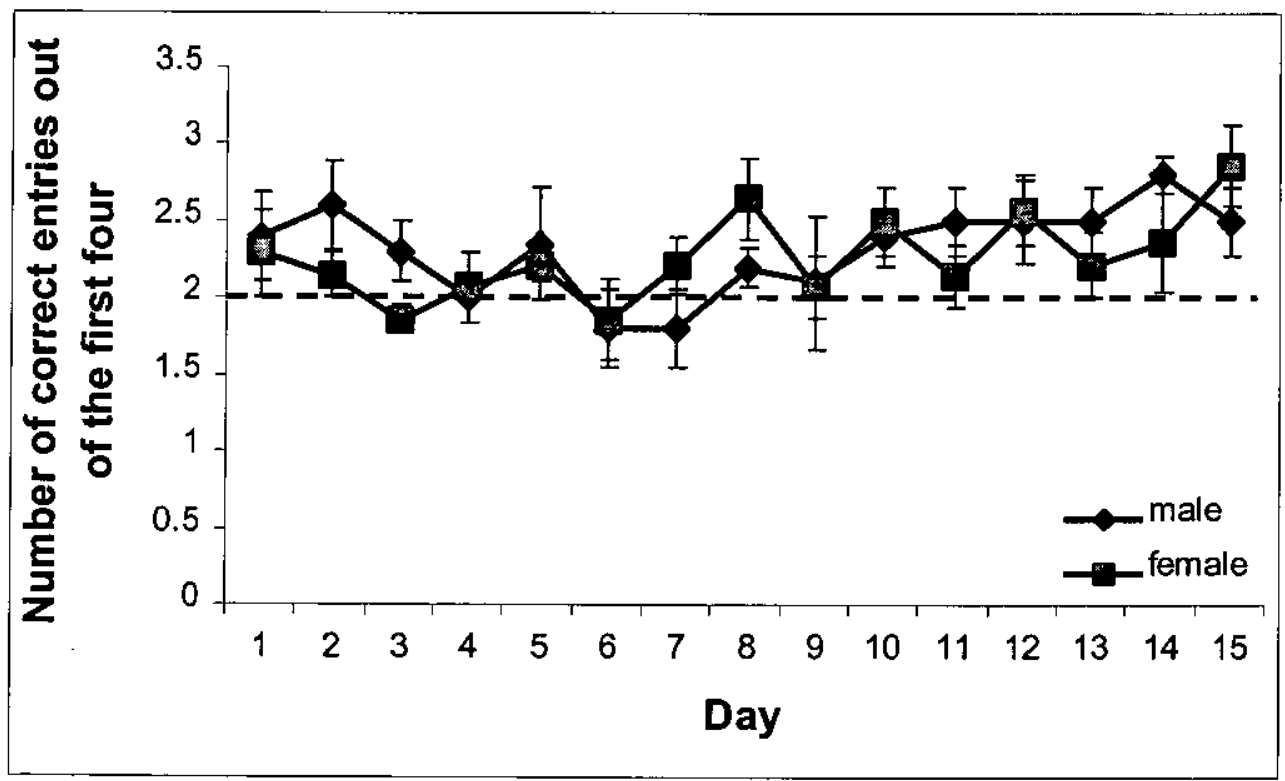




\subsubsection{Discussion}

\section{Working memory task}

As the gerbils did not consistently eat the rewards in the RAM working memory task I decided to analyse their spatial behaviour irrespective of whether the rewards were eaten. This assumed that they were motivated to explore the maze by something other than food. The gerbils spontaneously avoided previously visited arms. The subjects in my study entered more novel arms in the first eight choices than would be expected if they chose arms entirely at random, even though they did not consistently eat the food rewards.

This result replicated work with laboratory rats. Rats visit more novel arms in the RAM than expected by chance, even if they are not food deprived and the maze does not contain food rewards. Food-deprived rats in an unrewarded eight-arm RAM enter significantly more novel arms in their first eight choices than expected by chance, and perform as well as food-deprived rats in a rewarded maze (Timberlake \& White, 1990). Non-deprived rats in an unrewarded eight-arm RAM also enter arms in a non-random pattern, suggesting that they remember and avoid arms they have recently visited (Fitzgerald et al., 1985; Wilkie et al., 1992). Wilkie et al. (1992) gave non-deprived rats one trial a day in an unrewarded RAM for 36 days, and found that the 'patrolling' behaviour did not decrease over time. These studies suggest that rats use their working memory to spontaneously avoid places recently visited and that this results in efficient traversal of the RAM even in the absence of food motivation.

There were no differences between the male and female gerbils' explorationmotivated working memory behaviour in this study. There were also no sex differences in motivation, as measured by the average number of rewards eaten during a trial, on either task. There were only sex differences in the average time spent in each arm on days 5 and 6 , when males spent longer in each arm than females. On the other seven days there were no sex differences in the average time spent in each arm, which replicates the findings of Mead et al. (1995) who found no 
sex differences in spontaneous locomotor activity in gerbils tested in an automated activity monitoring system.

\section{Reference memory task}

Despite often ignoring the food, two lines of evidence suggest that the gerbils learned the reference memory task. First, they entered more novel rewarded arms during the first four arm choices on the last day of training than would be expected if they were randomly choosing arms without replacement, their choices did not differ from random on the first day of the experiment, when they were already performing well on the working memory task. Second, the gerbils made significantly fewer reference memory errors on day 15 than on day 1 . These results suggest that the gerbils were motivated by the food rewards, and by the end of the training had learnt the location of the rewarded arms (i.e. the reference memory task). The gerbils in my study reached a level of performance roughly equivalent to that described in a previous study where control (un-manipulated) male gerbils where trained on a RAM task with five out of eight arms rewarded (Liang et al., 1997).

In my study there was no sex difference in the number of reference memory errors made, but females tended to make fewer working memory errors than males. This non-significant trend is in the opposite direction to that predicted by the range size hypothesis and hippocampus size data.

The results of this study are inconclusive because the gerbils did not consistently eat the food rewards in the maze. Although the food rewards were palatable, the gerbils may not have been sufficiently food deprived. Previous studies testing gerbils in the RAM have used target weight food restriction, which involves the calculation of a target weight as a percentage of unrestricted weight. The subjects were put on restricted diets and weighed regularly (e.g. Katoh et al., 1992; Amano et al., 1993; Liang et al., 1997; Schwartz-Bloom et al., 1998). However, I was wary about using this form of food restriction because of possible welfare problems. Gerbils are much smaller than rats, which means that percentage weight loss may be difficult to maintain accurately and which in turn may result in underweight subjects. A second 
concern was that the subjects in this experiment were housed in groups of four and so a dominant individual might have monopolised the food ration resulting in severe weight loss in less dominant cage-mates.

The gerbils were next tested in the MWM, a spatial test that is aversively motivated and so may be expected to give different results to the appetitive-motivated RAM.

\subsection{Morris water maze task}

\subsubsection{Materials and methods}

The same subjects were used as in the RAM study; they were housed under the conditions described above. One female subject was excluded from all MWM training due to a physical deformity that might have affected its swimming ability. The subjects were identified by coloured ink on their tails to identify each of the four cages, and ear-punches to identify each of the four gerbils within a cage. The tail marks were reapplied every 3-4 days. The subjects had their ears punched on $5^{\text {th }}$ April. The MWM experiment was carried out from $23^{\text {rd }}$ April $-13^{\text {th }}$ May 2002. The subjects were approximately 22 weeks old at the start of the MWM training, the males weighed between $64 \mathrm{~g}$ and $73 \mathrm{~g}$ (mean $67 \mathrm{~g}$ ), and the females weighed between $53 \mathrm{~g}$ and $72 \mathrm{~g}$ (mean $65 \mathrm{~g}$ ).

The same room was used for the MWM task as for the RAM training. The water maze used to test the gerbils was a circular blue plastic children's paddling pool (Intex Recreation Corp., $255 \mathrm{~mm}$ high, $1.2 \mathrm{~m}$ diameter). This was placed on the wooden platform inside the MWM that the RAM had previously stood on. As for the RAM training, extra visual cues were fixed to the inner wall of the large MWM. Every effort was made to keep the objects in the room that may have provided spatial cues for the gerbils in a constant position throughout the experiment.

\section{Pretraining}

Pretraining was designed to familiarise the subjects with the MWM and allow them to gradually learn to swim (Von Lubnitz et al., 1996; Kondo et al., 1997). Fairly 
extensive pretraining was necessary because gerbils are prone to seizures when introduced to novel situations and have to be slowly familiarised with the water, rather than swimming spontaneously as rats do. Each session consisted of one swim for each subject given in a pseudorandom order, so that approximately equal numbers of each sex swam in the first and second halves of the session (exactly equal numbers could not be achieved as there were more males than females).

No platform was present in the maze during pretraining, and clear water at $27^{\circ} \mathrm{C}(+/-$ $1^{\circ} \mathrm{C}$ ) was used. The subjects were introduced to the maze from release positions that were constant for all animals within a session but varied between sessions in a pseudorandom way, so that each of the four release positions was never used more than once each day. After each $60 \mathrm{sec}$ swim the subject was removed, towel dried, and put under a heat lamp for $5 \mathrm{~min}$, to allow it to warm up and be monitored for adverse effects, before being returned to its home cage.

The subjects were closely monitored while they were in the MWM. If a gerbil had a seizure it was removed from the maze (this only happened twice, both times on the first day). The water depth was increased gradually. While the water was still so shallow that the subjects could touch the bottom of the maze (110 mm or less), they sometimes jumped out of the maze. When this happened they were placed back in the water. The subjects were unable to escape from the maze when the water was at its final depth of $165 \mathrm{~mm}$.

Pretraining took six days, as detailed below. There was a break of two days between pretraining days 1 and 2, and a break of one day between days 3 and 4 .

Day 1. Two sessions with water depth $30 \mathrm{~mm}$, at this depth the subjects paddled.

Day 2. One session with water depth $30 \mathrm{~mm}$ followed by two sessions with water depth $70 \mathrm{~mm}$, at which depth the subjects could still stand up but began to swim.

Day 3. One session with water depth $70 \mathrm{~mm}$ followed by two sessions with water depth $110 \mathrm{~mm}$, at which depth subjects could still touch the bottom with their hind legs. 
Day 4. Two sessions with water depth $110 \mathrm{~mm}$ followed by one session with water depth $165 \mathrm{~mm}$, at which depth the subjects had to swim.

Days $5 \& 6$. Four sessions with water depth $165 \mathrm{~mm}$.

Between sessions faeces were removed from the maze with a net. At the end of each day the paddling pool was drained and allowed to dry out. The final pretraining trial was recorded on videotape to provide baseline data on swim speed.

\section{Main experiment}

Only gerbils that were able to swim freely in the maximum depth $(165 \mathrm{~mm})$ for $60 \mathrm{sec}$ were included in the experiment. Six females and six males took part in the main experiment. All of the trials were recorded on videotape. The same MWM was used as was used for the pretraining. It was filled with $27^{\circ} \mathrm{C}\left(+/-1^{\circ} \mathrm{C}\right)$ water to a depth of $165 \mathrm{~mm}$. $500 \mathrm{~g}$ of coffee whitener (Sainsburys Coffeeplus) was dissolved in warm water and added to the maze.

The platform was painted white and measured $155 \mathrm{~mm}$ high with a diameter of $110 \mathrm{~mm}$. It was located in the NW quadrant, $250 \mathrm{~mm}$ from the side of the maze, throughout the experiment. The release position was constant for all individuals within a session, each position used once a day in a random order. The water was stirred between each subject to eliminate odour trails that the subjects could possibly follow (Means et al., 1992).

Four sessions of trials were run each day for 10 consecutive days. A session consisted of one trial for each subject. The sessions started at 1000, 1130, 1330 and $1500+/-30 \mathrm{~min}$. The gerbils were allowed to swim until they climbed onto the platform, or for a maximum of $60 \mathrm{sec}$. Climbing onto the platform, rather than just touching it, was chosen as the endpoint of the trials because it demonstrated that the subject knew where the platform was. This is a commonly used endpoint for such an experiment (Shuaib et al., 1996; Li et al., 1999; Iqbal et al., 2002). If the subject touched the platform and continued swimming it was unclear whether it just bumped into the platform by accident and was not looking for it, or whether the subject was 
searching for the platform but was unable to climb onto it. No subject was consistently unable to climb onto the platform. If the subject did not climb onto the platform within $60 \mathrm{sec}$ the experimenter guided it to the platform. Once on the platform the subject was left there for $15 \mathrm{sec}$ before being removed, towel dried, put under a heat lamp and returned to its home cage.

On the day immediately following the final day of MWM training a probe trial was carried out. The platform was removed from the maze and each subject was allowed to swim for $60 \mathrm{sec}$.

\subsubsection{Results and analysis}

A repeated-measures analysis was carried out on the latency to climb on to the platform blocked by day (one day was the mean of four trials), using the following GLM model:

Latency $=\operatorname{sex}+$ gerbil (sex $)+$ day + sex $*$ day

The interaction term was not significant and was removed from the analysis. The effect of day was significant $\left(\mathrm{F}_{9,99}=5.92, \mathrm{p}<0.001\right)$. The latency to climb onto the platform decreased over time (figure 6.6). There was no significant difference between the sexes (figure 6.6; $\mathrm{F}_{1,10}=2.36, \mathrm{p}=0.155$ ).

Swim speed was calculated for each subject by measuring the distance that they travelled during the first $30 \mathrm{sec}$ of the last pretraining trial. This was measured by tracing the route taken by the subject onto a transparent sheet attached to the monitor. The length of this trail was then measured using a map measurer, and the distance left in arbitrary units. The GLM model 'speed = sex' revealed no sex differences in swim speed (figure $6.7 ; \mathrm{F}_{1,10}=0.57, \mathrm{p}=0.469$ ). 
Figure 6.6: Latency to climb onto the hidden platform during Morris water maze training, by sex and day. Male $n=6$, female $n=6$. Each day is the mean of four trials. $+/$ SEM.

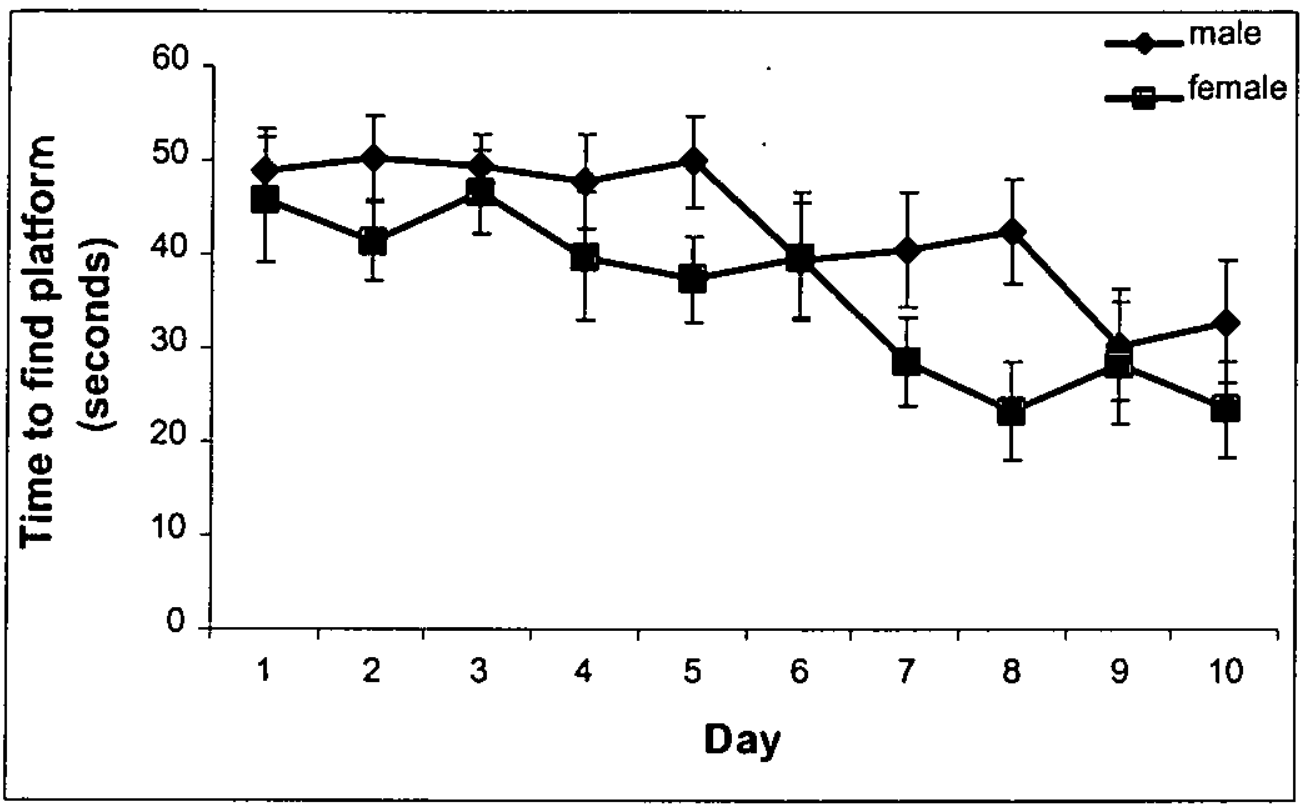

Figure 6.7: Distance swum (in arbitrary units) by the male and female gerbils during $30 \mathrm{sec}$ in the last Morris water maze pretraining trial. Male $n=6$, female $n=6$, + - SEM.

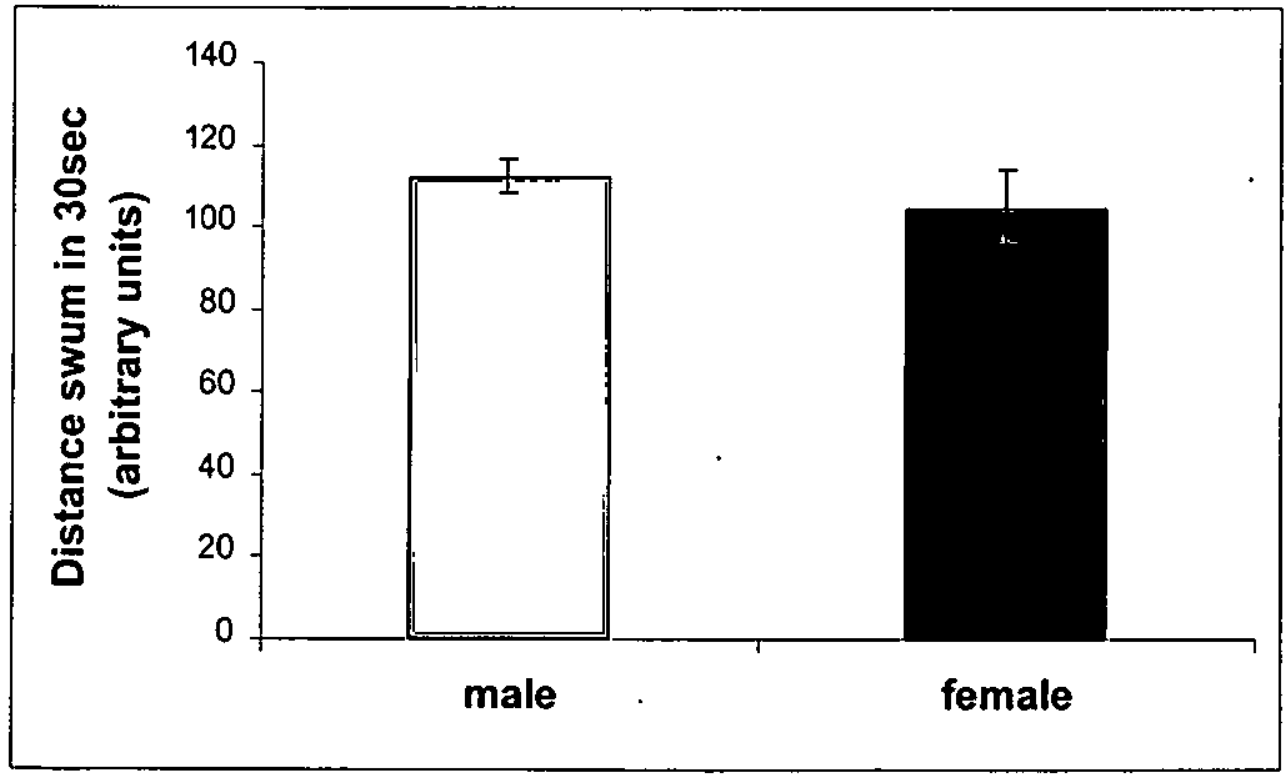


Chapter 6: Sex differences in spatial ability and the hippocampus in gerbils?

The time that each subject spent in the quadrant that had previously contained the platform during the probe trial ('correct' quadrant) was also recorded. The GLM model 'time $=$ sex' revealed no sex differences in this measure of spatial ability (figure 6.8; $\mathrm{F}_{1,10}=0.06, \mathrm{p}=0.815$ ). One-sample $\mathrm{t}$-tests showed that the female subjects spent more time in the correct quadrant than the random expectation of $15 \mathrm{sec}(n=6$, $\mathrm{t}=3.51, \mathrm{p}=0.017$ ). The male subjects tended to spend more time than expected by random in the correct quadrant $(\mathrm{n}=6, \mathrm{t}=2.33, \mathrm{p}=0.068)$. This effect became significant when a single outlying data-point was removed $(n=5, t=4.24, p=0.013)$. The subject whose data-point was removed spent only $4 \mathrm{sec}$ in the correct quadrant, the remaining five subjects spent between 21 and $44.5 \mathrm{sec}$ in the correct quadrant. Removal of this data-point did not change the significance of the effect of sex on time spent in the correct quadrant.

Figure 6.8: Time spent in the correct quadrant during the Morris water maze probe trial. Dashed line shows random performance of $15 \mathrm{sec}$. Male $n=6$, female $n=6,+/-$ SEM.

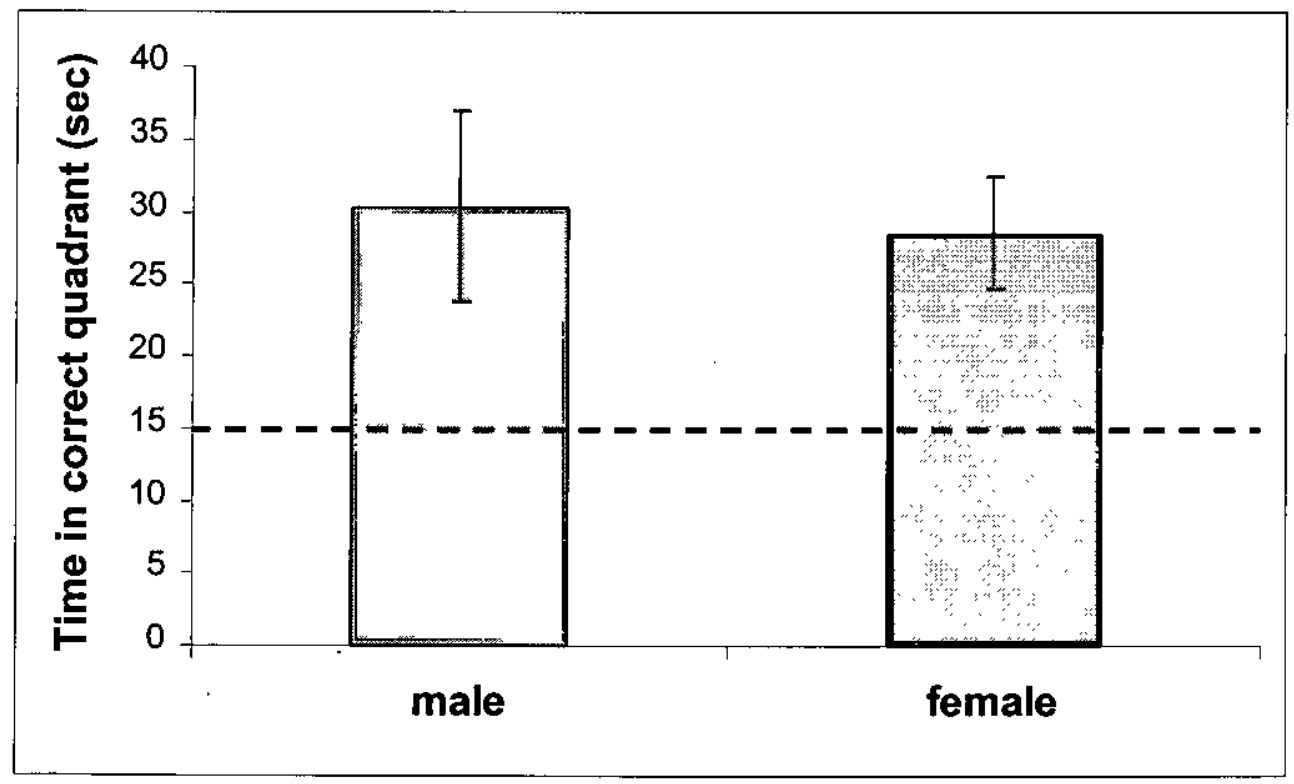




\subsubsection{Discussion}

There was no sex difference in the spatial ability of gerbils measured using the MWM. However, the gerbils did spend more time than would be expected by chance in the quadrant that had previously contained the platform during the probe trial. This suggests that they learnt the task.

The performance shown by un-manipulated (control) gerbils in other studies that have tested this species on the hidden platform MWM task tends to be better than the performance reached by my subjects. For example, Iqbal et al. (2002) trained male gerbils in the MWM, giving them five trials a day for seven days in a similar sized maze to that used in my study. The gerbils in Iqbal et al.'s (2002) study had learnt to climb onto the platform within a mean of $20 \mathrm{sec}$ by the third day of the study. This performance is better than that seen in my experiment (the males found the platform within a mean of $33 \mathrm{sec}$ on the $10^{\text {th }}$ day; figure 6.6), but may be explained by the larger platform used in Iqbal's study, which would have been easier to find $(220 \mathrm{~mm}$ diameter compared to $110 \mathrm{~mm}$ in my experiment). Zhou et al. (2001) gave gerbils three trials per day for five days, using a similarly sized MWM and platform to that used in my study (they do not state the gerbils' sex). On the last day of training the gerbils found the platform within an average of $20 \mathrm{sec}$. This performance is again better than that seen in my study. The two studies quoted above both gave their subjects all of their daily trials with shorter inter-trial intervals than were used in my study (Iqbal et al., 2002: 30min; Zhou et al., 2001: 30min; my study: approx. 1.5hr). This difference may explain why my gerbils did not learn as well as those in the other studies.

The results from the MWM study did not show superior male spatial ability, as predicted by the range size hypothesis and sex differences in hippocampus size. As only four male and five female subjects took part in both the water and RAM studies, the planned regressions to look for correlations between performance in the two mazes were not carried out. However, the similar results from the two studies suggest that the two tasks may be testing the same thing, despite the differences in 
motivation and training regime. This is somewhat surprising as the MWM is not an obviously appropriate spatial test for gerbils.

Mongolian gerbils are desert animals and would probably have few chances to swim in the wild. Unlike rats, they do not swim spontaneously and have to be introduced to water gradually. The MWM is a stressful spatial test, and laboratory mice and short-tailed opossums (Monodelphis domestica) have been shown to perform less well on it than rats, even though they perform as well as rats on spatial tasks such as the RAM (Kimble \& Whishaw, 1994; Whishaw \& Tomie, 1996). I do not have a control group of rats tested under the same conditions as the gerbils and so I am unable to directly compare my gerbils' performances on the two tasks, but they did not appear to be greatly impaired on the MWM relative to the RAM.

Although pre-training in a water maze without a platform, and in the RAM with the rewards in the central compartment, is fairly common in studies of sex differences in spatial ability (e.g. Juraska et al., 1984; Roof, 1993b; Von Lubitz et al., 1996; Isgor \& Sengelaub, 1998), it is possible that this might account for the very slow learning displayed by the gerbils in these two maze tasks. During pre-training, subjects learn about the reward (i.e. that the food is in the centre of the RAM, and that the only means of escape from the MWM is to swim until rescued) and this information will have to be relearned when the main experiment starts and the food is at the end of the RAM arms, or the platform provides a quick escape from the MWM. The pretraining methods should probably be altered in future experiments in order to avoid this apparent conflict.

It is possible that sex differences in spatial ability were not found because the additional landmarks affixed to the sides of the large MWM made the task easier for the females and improved their performance to male levels. There is evidence from humans, rats and kangaroo rats that females depend more on nearby landmark cues to solve spatial tasks, while males tend to use global geometric cues such as the shape of the room (e.g. Langley, 1994; Dabbs et al., 1998; Kanit et al., 1998a; Montello et al., 1999). There is no evidence that this is also true for gerbils, but it seems to be a general effect and so is a possibility. The type and stability of cues 
provided can affect whether or not sex differences are found in a task. For example, Roof and Stein (1999) did not find sex differences in the performance of rats in the MWM when the experimenter provided a stable landmark cue, but the female rats performed worse than the males when the experimenter did not always stand in the same position and so was an unreliable landmark.

Another possible reason why sex differences in spatial ability were not found, is that the sex difference in gerbil range size is not biologically significant, and therefore has had no effect on the evolution of spatial ability. In Agren et al.'s (1989) study of the social behaviour of wild Mongolian gerbils, gerbils were found to have a promiscuous mating system, and live in mixed-sex groups of individuals that shared a home territory and burrow system. Each group typically consisted of a malefemale pair plus offspring. The males in a group ranged over approximately twice as large an area as the females in the group visited. In Gaulin and Fitzgerald's original (1986) study the male meadow voles had ranges that were on average three times larger than the females', while in their later study the male meadow voles had home ranges that were nearly seven times as large as the females' (Gaulin \& Fitzgerald, 1989). It may be that the difference in range size between male and female gerbils is not biologically significant. It is also possible that there are only sex differences in range size in the population studied by Agren et al. (1989), and not in the species as a whole. Such intraspecific variation has been found in prairie voles: Gaulin and Fitzgerald (1989) did not find a difference between the range sizes of male and female prairie voles, but Swihart and Slade (1989), measuring the range sizes of a different population, found that males had ranges that were approximately twice as big as those of females. Gaulin and Fitzgerald $(1986 ; 1989)$ measured the range size and spatial ability of a single population of each species. The population used in my study and that tested by Agren et al. (1989) were not the same, not least because my gerbils were domesticated.

My next step was to examine the hippocampal volumes of my sample of gerbils. This was done in order to see if Sherry et al.'s (1996) finding that male gerbils have larger hippocampi than females could be replicated in this sample, despite the lack of 
sex differences in spatial ability, and to look for correlations between hippocampus parameters and spatial ability (Roof \& Havens, 1992; Roof, 1993a).

\subsection{Brain measurements}

\subsubsection{Materials and methods}

Three days after the MWM experiment finished (15 $5^{\text {th }}$ May 2002) the eight male and eight female gerbils used in the maze experiments were sacrificed by an overdose of $\mathrm{CO}_{2}$. After death was confirmed they were decapitated and their heads were placed in individual, labelled jars of $4 \%$ formalin for one week. The brains were then removed and again placed in individual, labelled jars of $4 \%$ formalin. Approximately three weeks later the brains were sliced into $30 \mu \mathrm{m}$ coronal sections from the anterior of the brain to the posterior of the hippocampus, using a cryostat. Every $5^{\text {th }}$ section was mounted onto a gelatin-coated slide and stained with $0.1 \%$ cresyl violet. This resulted in mounted sections at $150 \mu \mathrm{m}(0.15 \mathrm{~mm})$ intervals. Each slide was re-labelled with a dummy code so that I was blind to the sex of each subject. Each section was magnified by approximately 11 times (the magnification was kept constant for all the slides) using an Olympus SZX12 microscope with an Olympus ACH1X lens, and photographed with a digital camera (Olympus Camedia C-2020Z). The photographs were saved as JPEG files.

\section{Hippocampal volume}

The resolution of the files was scaled down from $1600 \times 1200$ pixels to $900 \times 675$ pixels and converted to bitmap format using Adobe Photoshop. This produced files of the size and format preferred by the image analysis software. The areas of the right and left hippocampus and telencephalon excluding the hippocampus were measured on each section using Scion Image (version beta 4.0.2, Scion Corp.); the measurements were taken in square pixels and later converted to square millimetres. Loskota et al.'s (1974) gerbil brain atlas and Paxinos and Watson's (1997) rat brain atlas were used to define brain regions. The hippocampus included the fimbria, dentate gyrus, subiculum, alveus hippocampi, and sulcus hippocampi (after Sherry et al., 1996). The telencephalon included the cerebral cortex, olfactory bulb, fornix, 
corpus callosum and nucleus caudatus, but not the thalamus, hypothalamus or optic tracts (figure 6.9). I attempted to estimate the area of all the brain sections, but some sections were damaged and this was not possible. As I only had sections from the front of the brain until approximately the end of the hippocampus, I measured hippocampus and telecephalon areas from the anterior of the brain until the last section on which the hippocampus was visible, or until the end of the available sections, whichever came first.

Figure 6.9: A section of a gerbil brain highlighting the measured regions.

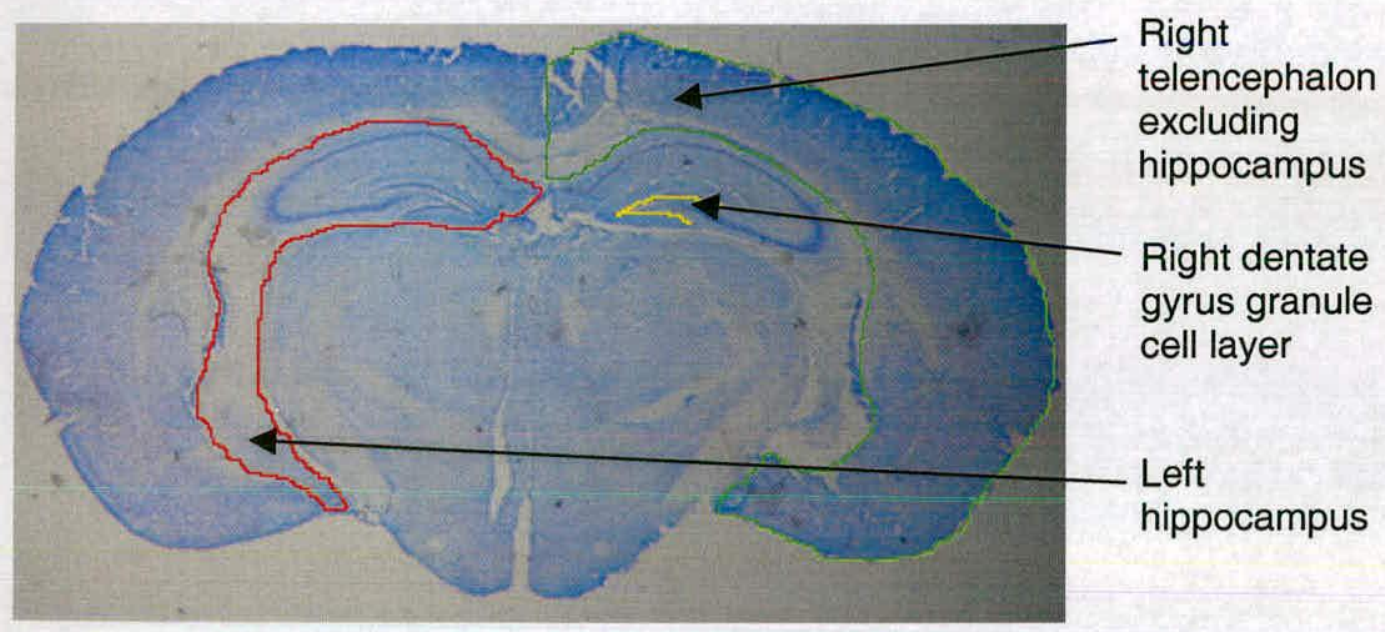

The volumes of the right and left hippocampus and right and left telencephalon were calculated for each subject using the equation to calculate the volume of a truncated cone:

$\mathrm{V}=\mathrm{I} / 3\left(\mathrm{~h}_{1}+\sqrt{\mathrm{h}_{1}} * \sqrt{ } \mathrm{h}_{2}+\mathrm{h}_{2}\right)$

Where $h_{1}$ and $h_{2}$ are areas from consecutive sections and $I$ is the interval between the sections.

As the posterior ends of the brain regions were not always present in the sections the volumes of the posterior end slices were not calculated. However, the volumes of the anterior end slices were calculated. When sections were missing the volume was 
calculated between the available sections. If the areas of end sections were missing (but it was apparent from the slides that the brain regions were still present in these sections) then the area of the end section was assumed to be the same as the last available area measurement. A mean of 19.8 (range 18-23) sections were analysed per subject to determine the hippocampal volume, and a mean of 37.1 (range 33-40) sections for the telencephalon.

\section{Dentate gyrus granule cell layer}

Following the method of Roof and Havens (1992) the section on which the posterior commissure first appeared (moving anterior to posterior) was designated section six and the five sections immediately anterior to section six were designated sections one to five. Keeping the original resolution, these six sections per subject were cropped and converted to bitmap files using Adobe Photoshop. The right and left DG-GCL widths were measured using Scion Image and converted into millimetres. As in Roof and Havens' (1992) study the mean of the left and right DG-GCL widths were calculated for each subject. However, some of the DG-GCL widths could not be measured because the sections were damaged. This caused a problem because the DG-GCL tends to increase in size moving anterior to posterior and missing sections may bias the data.

\subsubsection{Results and analysis}

\section{Hippocampal volume}

Volumes of the right and left hippocampus and telencephalon for the male and female subjects are shown in figure 6.10. The following GLM was applied separately to the hippocampus and telencephalon volumes:

Volume $=\operatorname{sex}+$ gerbil $($ sex $)+$ hemisphere + sex*hemisphere

This model was equivalent to a paired t-test allowing for sex. The interaction term was not significant and was removed from the final analysis. The data were tested for the parametric test assumptions of normality of error and homogeneity of residuals, in order to meet these the telencephalon volume data were cubed. The 
Chapter 6: Sex differences in spatial ability and the hippocampus in gerbils?

results indicated that there was no difference in the volume of the right and left sides of either brain region (hippocampus: $\mathrm{F}_{1,15}=1.00, \mathrm{p}=0.333$; telencephalon: $\mathrm{F}_{1,15}=2.52$, $\mathrm{p}=0.134$ ), therefore all further analysis was carried out on the total (left plus right) volume.

Hippocampal volume was then analysed relative to telencephalon volume using the following GLM:

Hippocampus $=\operatorname{sex}+$ telencephalon $+\operatorname{sex} *$ telencephalon

Telencephalon volume was included as a covariate. The parametric assumptions were met. The interaction term was not significant and was left out of the final model. Neither sex nor telencephalon volume had a significant effect on hippocampal volume (sex $\mathrm{F}_{1,13}=0.11, \mathrm{p}=0.742$, telencephalon $\mathrm{F}_{1,13}=0.33, \mathrm{p}=0.578$; figure 6.11).

Figure 6.10: Volumes of the brain regions, by sex and hemisphere. +/- SEM. Male $n=8$, female $n=8$.

a) Hippocampal volume.

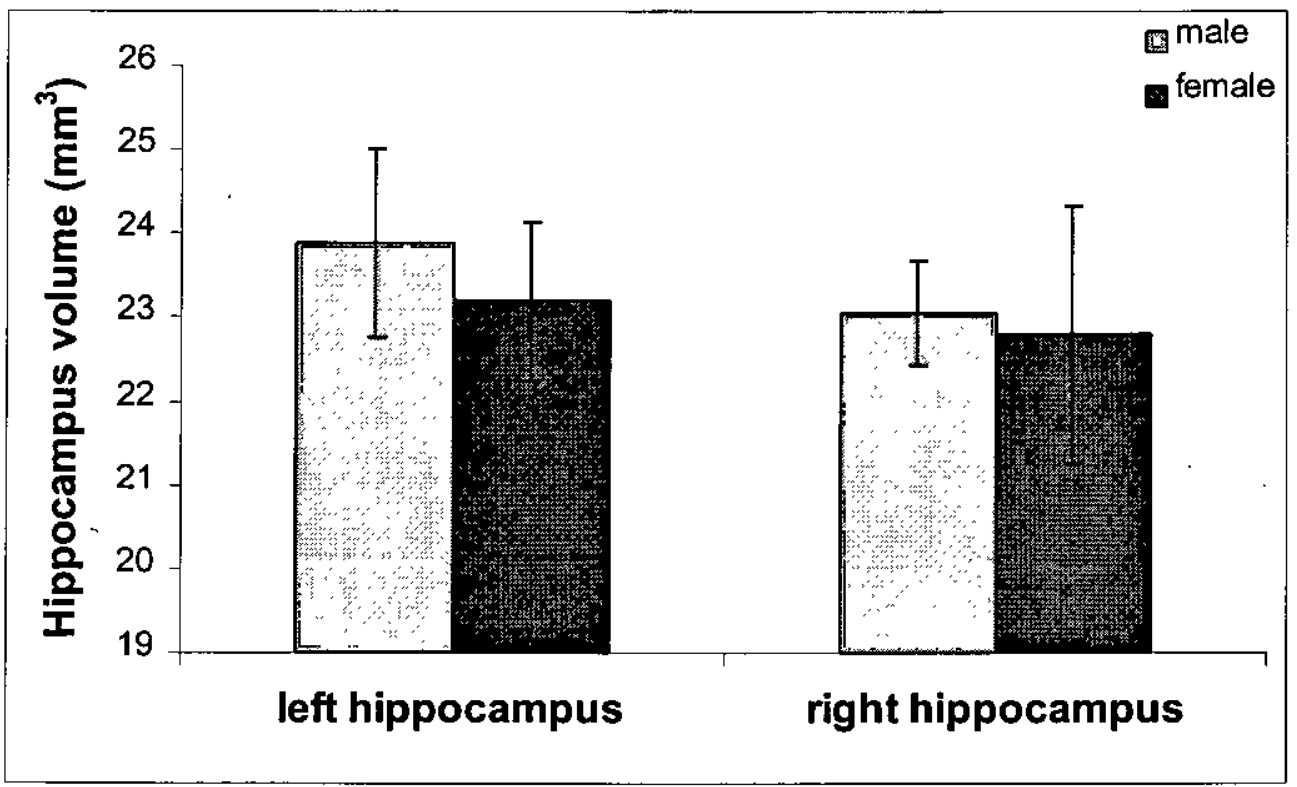


b) Telencephalon volume.

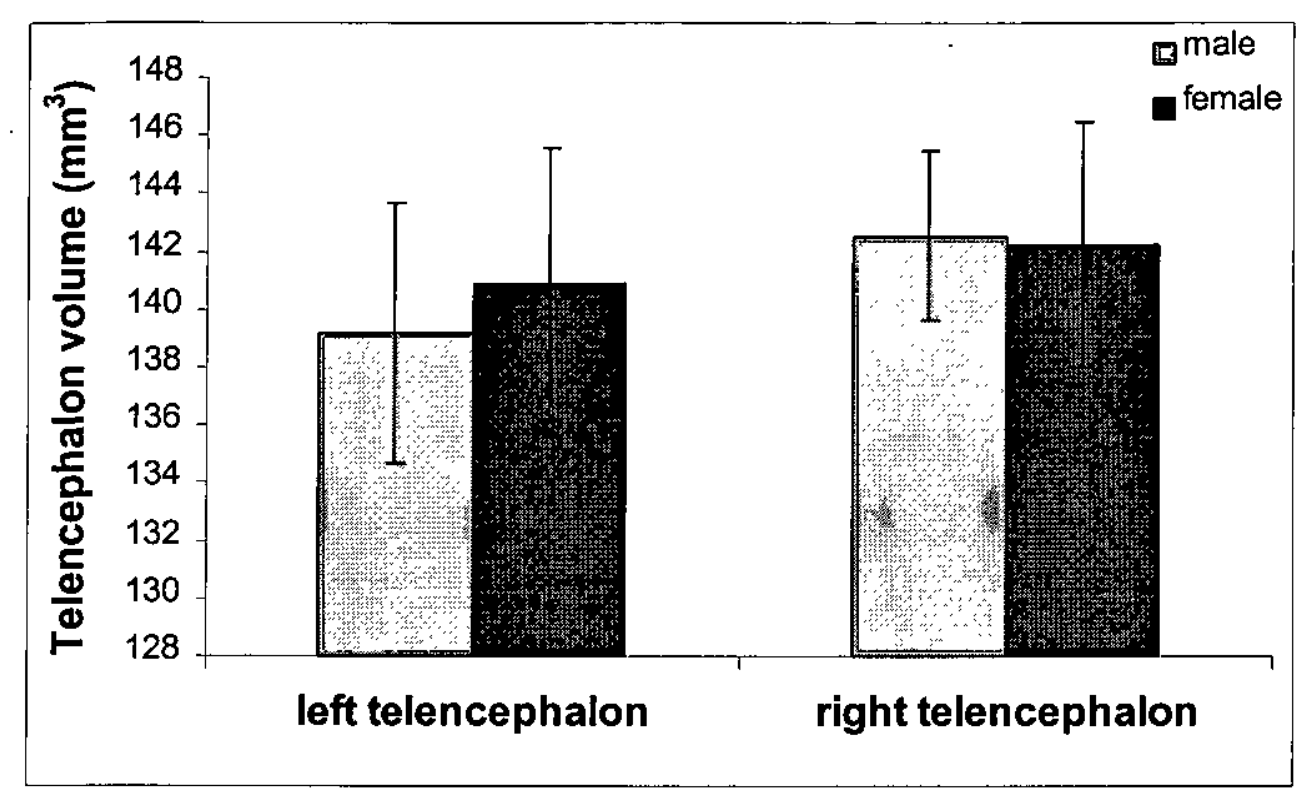

Figure 6.11: Hippocampal volume relative to the telencephalon volume of the male and female gerbils.

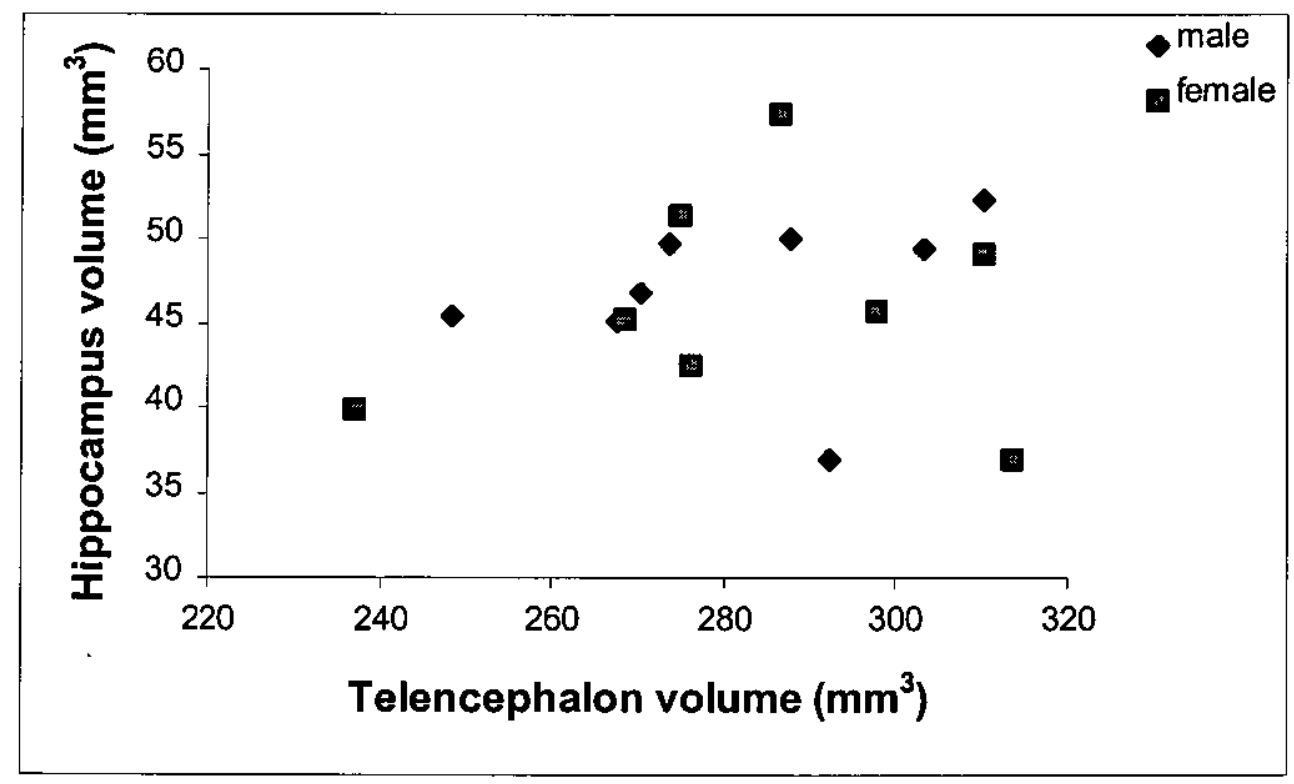




\section{Dentate gyrus granule cell layer}

The mean width of the available sections was calculated for each individual and the data were analysed using a repeated-measures ANOVA with 'sex' as the betweensubjects effect and 'hemisphere' as the within-subject effect. The interaction term was not significant and was removed from the model. The data met the parametric assumptions. The effect of sex was not significant $\left(\mathrm{F}_{1,14}=1.14, \mathrm{p}=0.305\right)$. The effect of hemisphere was approaching significance $\left(F_{1,15}=3.12, p=0.098\right)$, this seems to be due to a tendency for the left DG-GCL to be wider than the right one (figure 6.12). When the data were reanalysed using only the eight subjects that had a complete set of measurements the results did not change in significance ( $\operatorname{sex} F_{1,6}=2.87, p=0.142$, hemisphere $F_{1,7}=3.78, p=0.093$ ).

Figure 6.12: Dentate gyrus granule cell layer width, by sex and hemisphere. +/SEM. Male $n=8$, female $n=8$.

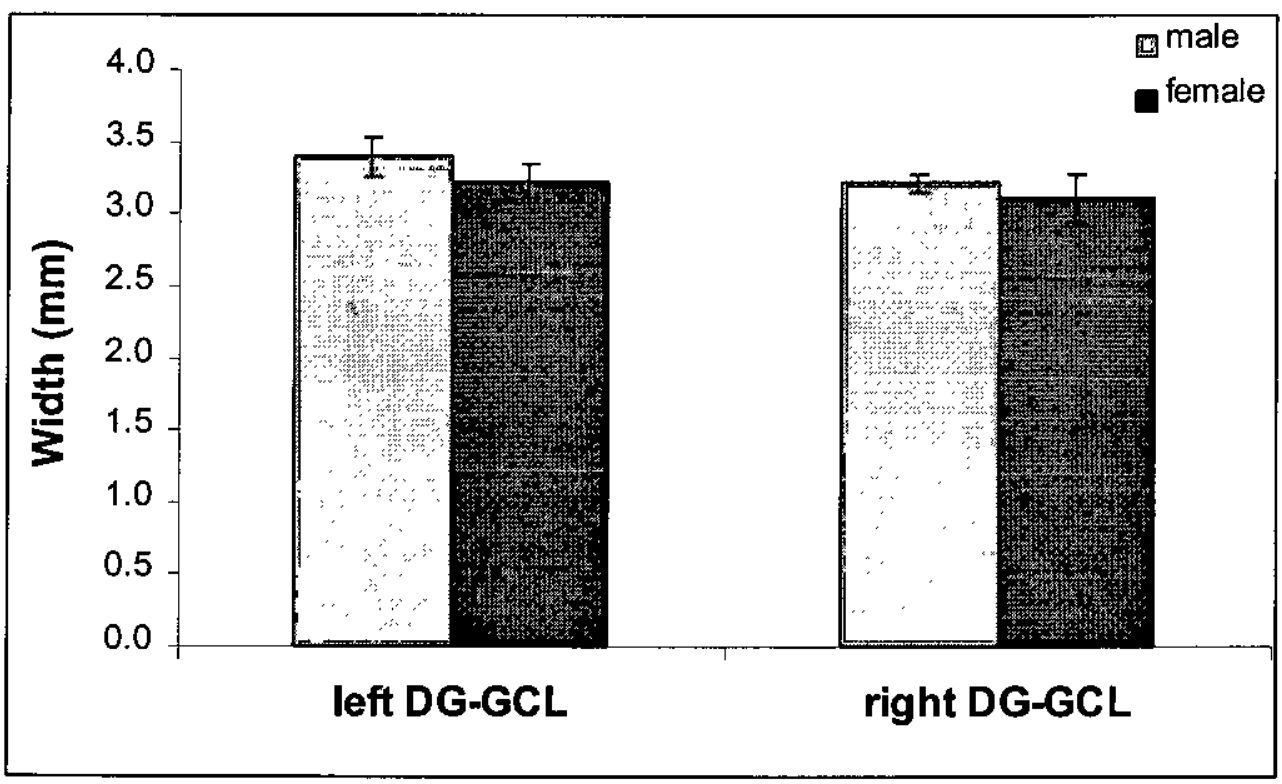




\section{Regressions}

Five measures of spatial ability were regressed against the mean left, mean right, and overall mean (of all 12 sections, both left and right) DG-GCL width, allowing for sex, using the GLM 'response $=$ sex + width + sex* width', where width was a covariate. Both right and left DG-GCL widths were used, despite there being no significant difference between them, because the correlation found by Roof and Havens (1992) was specifically between the right DG-GCL width and water maze performance. Subjects that did not have a complete set of measurements were included in the analysis because no spatial ability data was available for some of the subjects. If subjects with missing DG-GCL measurements were excluded the sample sizes would have been very small. The measures of spatial ability used were as follows:

1) Mean number of novel arm entries in the first eight choices during the working memory RAM task (all subjects).

2) Mean number of reference memory errors made during the reference memory RAM task (five males and seven females).

3) Mean number of working memory errors made during the reference memory RAM task (five males and seven females).

4) Mean latency, in seconds, to find the platform during the MWM training (six males and six females).

5) Time, in seconds, spent in the correct quadrant during the MWM probe trial (six males and six females).

See table 6.3 for the F-ratios. There were only two significant effects. Firstly, the sex by width interaction term was significant for the mean number of working memory errors made during the reference memory RAM task when either the left or mean, but not the right DG-GCL width was analysed (left $\mathrm{F}_{1,8}=18.39, \mathrm{p}=0.003$; mean $\mathrm{F}_{1,8}=12.53, \mathrm{p}=0.008$; right $\mathrm{F}_{1,8}=0.48, \mathrm{p}=0.507$ ). This seems to be due to a positive 
Chapter 6: Sex differences in spatial ability and the hippocampus in gerbils?

correlation between DG-GCL width and the number of working memory errors made only in the male subjects (figure 6.13.a). Secondly, the effect of width (but not the interaction term or the effect of sex) was significant for the mean latency to find the platform during the MWM training when either the right or mean, but not left DGGCL width was analysed (right $\mathrm{F}_{1,9}=5.39, \mathrm{p}=0.045$; mean $\mathrm{F}_{1,9}=9.42, \mathrm{p}=0.013$; left $\mathrm{F}_{1,9}=3.37, \mathrm{p}=0.100$ ). This seems to be due to subjects with wider DG-GCLs taking longer to find the platform in the MWM (figure 6.13.b).

The above analysis was repeated using right, left and total hippocampal volume instead of DG-GCL width. See table 6.4 for the F-ratios. The only significant effect was that the sex by volume interaction term was significant when the right hippocampal volume was regressed against the time spent in the correct quadrant during the MWM probe trial $\left(\mathrm{F}_{1,8}=6.22, \mathrm{p}=0.037\right)$. This seems to be due to a positive correlation between right hippocampal volume and time spent in the correct quadrant during the MWM probe trial only in female subjects (figure 6.14).

As the regression analysis involved generating multiple p-values Bonferroni's adjustment was applied (Samuels, 1989). Using a significance level of 0.05 and looking at $90 \mathrm{p}$-values (tables $6.3 \& 6.4$ ), a p-value is not sufficient evidence of an effect unless it is smaller than 0.0005 . None of the p-values in the regression analysis were smaller than 0.001 . 
Chapter 6: Sex differences in spatial ability and the hippocampus in gerbils?

Figure 6.13: Correlation between mean dentate gyrus granule cell layer width and measures of spatial ability.

a) Mean working memory errors during the reference RAM task.

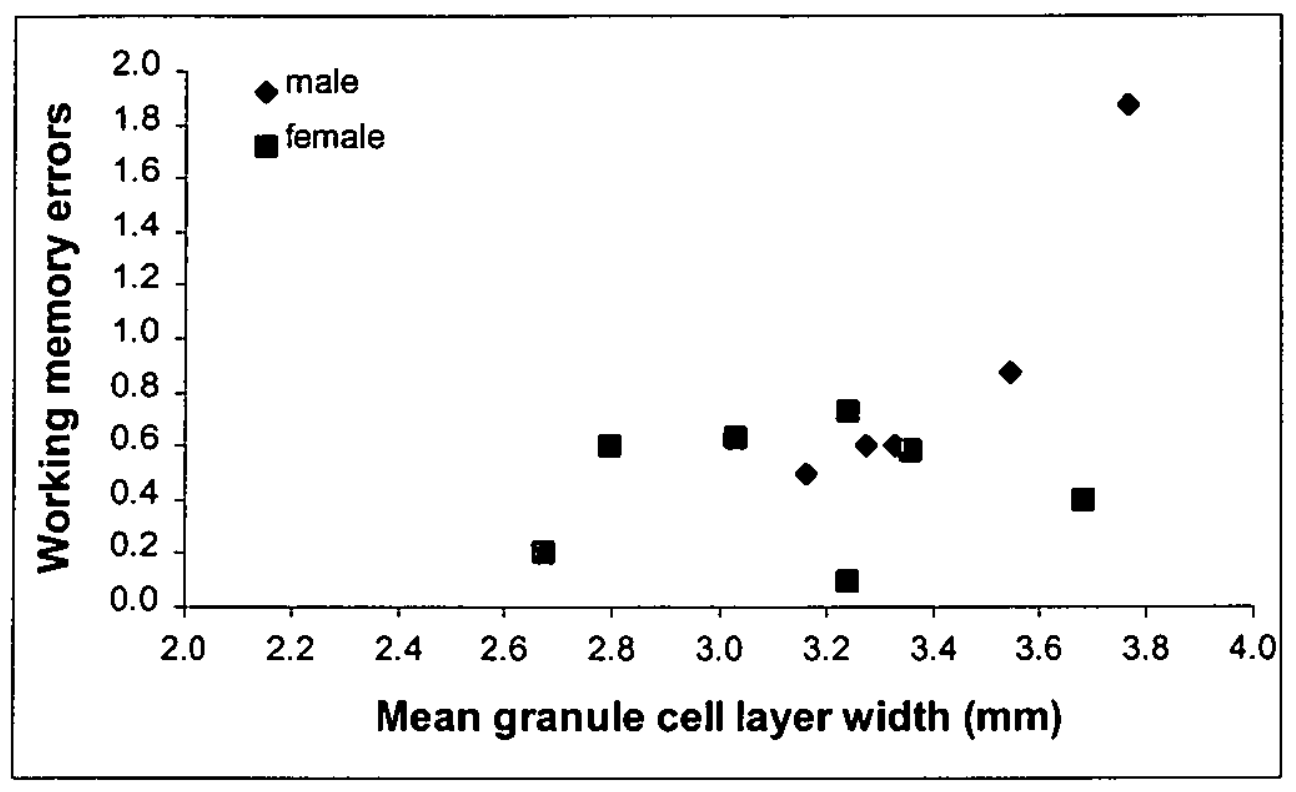

b) Mean latency to climb onto the hidden platform during MWM training.

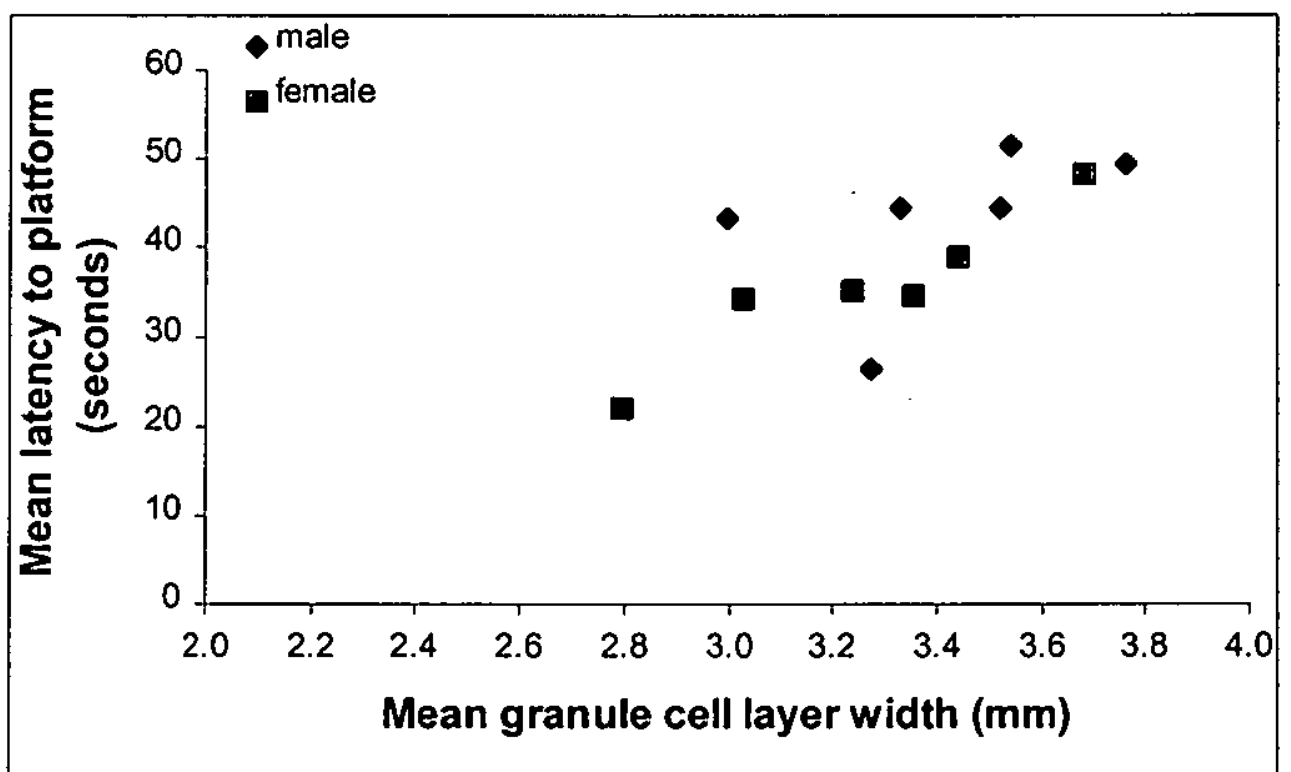


Chapter 6: Sex differences in spatial ability and the hippocampus in gerbils?

Figure 6.14: Correlation between right hippocampal volume and time spent in the correct quadrant during the MWM probe trial.

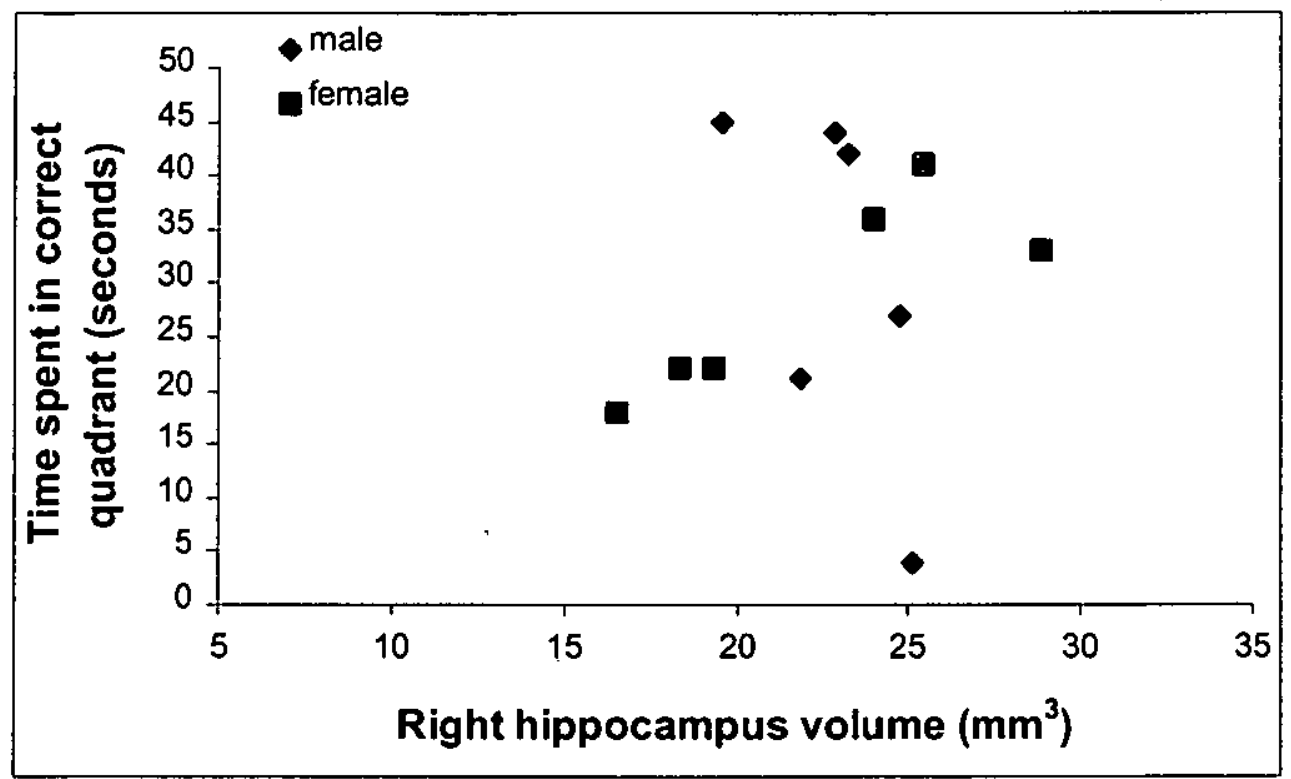


Table 6.3: F-ratios for the effects of sex and DG-GCL width on measures of spatial ability. ${ }^{*}=$ significant at $p<0.05,{ }^{* \star}=$ significant at $p<0.01$.

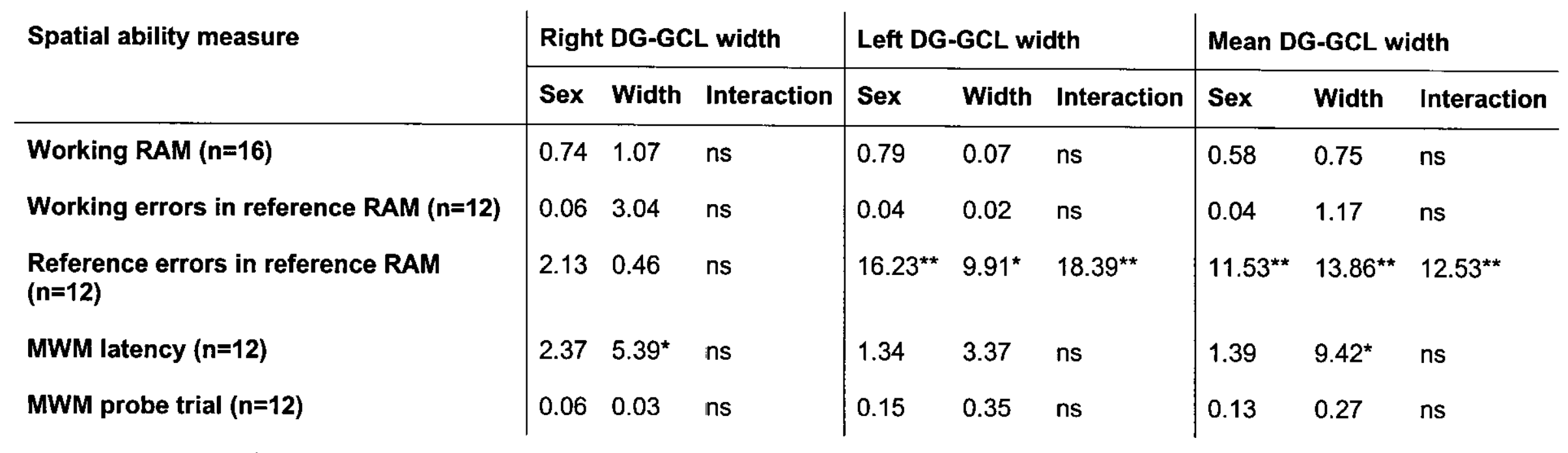


Table 6.4: F-ratios for the effects of sex and hippocampal volume on measures of spatial ability. ${ }^{*}=$ significant at $p<0.05$.

\begin{tabular}{|c|c|c|c|c|c|c|c|c|c|}
\hline \multirow[t]{2}{*}{ Spatial ability measure } & \multicolumn{3}{|c|}{ Right hippocampal volume } & \multicolumn{3}{|c|}{ Left hippocampal volume } & \multicolumn{3}{|c|}{ Total hippocampal volume } \\
\hline & Sex & Volume & Interaction & Sex & Volume & Interaction & Sex & Volume & Interaction \\
\hline Working RAM ( $n=16)$ & 0.97 & 0.42 & ns & 0.97 & $<0.01$ & ns & 0.93 & 0.14 & ns \\
\hline Working errors in reference RAM $(n=12)$ & 0.01 & 2.56 & ns & 0.03 & 2.21 & ns & $<0.01$ & 2.86 & ns \\
\hline $\begin{array}{l}\text { Reference errors in reference RAM } \\
(n=12)\end{array}$ & 2.80 & 0.10 & ns & 2.08 & 0.65 & $\mathrm{~ns}$ & 2.53 & 0.28 & ns \\
\hline MWM latency $(n=12)$ & 2.55 & 0.65 & ns & 2.20 & 0.07 & ns & 2.40 & 0.34 & ns \\
\hline MWM probe trial $(n=12)$ & $6.26^{\star}$ & 1.51 & $6.22^{*}$ & 0.07 & 0.07 & ns & 0.04 & 0.03 & ns \\
\hline
\end{tabular}




\subsubsection{Discussion}

There were no sex differences in hippocampal volume. This contradicts the results of Sherry et al. (1996), who found that male gerbils had larger hippocampal volumes than females. The sample sizes in this and Sherry's study were comparable (eight of each sex in this study, and nine males and 10 females in Sherry's study), so it is unlikely that I did not find sex differences simply due to small sample size. However, many of the sections that I measured were damaged and this may have contributed to the variation between individuals and possibly could have obscured any sex differences.

The subjects' age may also influence whether or not sex differences in hippocampal volume are found. Sherry et al.'s (1996) subjects were sacrificed at a mean age of 74.2 days, approximately the age at which gerbils reach sexual maturity. The subjects in this study were approximately 25 weeks old (175 days) when they were sacrificed. They were more than twice the age of Sherry et al.'s (1996) subjects, but were still well within the reproductive lifespan of a normal gerbil, as the average age for a female gerbil to have her last litter is 487 days (Norris, 1987). Brain regions including the hippocampus, have been found to grow at different rates in male and female rats (Diamond, 1987). As the subjects in Sherry et al.'s (1996) study were so young it is possible that the female gerbils' hippocampus grows more slowly than the males' and had not yet reached full size. This hypothetical scenario could result in the observed sex differences in hippocampus size in Sherry et al.'s young gerbils, but not in my fully mature adults. It could be tested by comparing the hippocampal volumes of male and female gerbils at a range of ages.

Whether or not subjects are in breeding condition may also affect whether or not sex differences in hippocampal volume are found. In meadow voles, sex differences in hippocampal volume are only found during the breeding season, when sex differences in spatial ability and range size are also apparent (Gaulin \& Fitzgerald, 1986; Galea et al., 1995; Galea et al., 1999). However, I have no reason to believe that the gerbils used in this study were not in reproductive condition, as gerbils breed throughout the year under laboratory conditions (Norris, 1987). 
The extensive spatial training that my gerbils underwent could have differentially affected the sexes' hippocampus size. There is evidence that spatial experience can affect the hippocampus at both the neuronal and gross morphological level: rats kept in a spatially enriched environment have increased dendritic spine density on hippocampus CA1 pyramidal cells compared to controls (Moser et al., 1994); similarly, seasonal spatial demands are correlated with an increase in neurogenesis in the hippocampus of black-capped chickadees and meadow voles (Barnea \& Nottebohm, 1994; Galea \& McEwen, 1999); marsh tits (Parus palustris) with experience of storing and retrieving food have larger hippocampi relative to the rest of the telencephalon and have more neurons in the hippocampus than controls with no storing experience (Clayton \& Krebs, 1994); finally, in humans, London taxidrivers (a spatially demanding job) have larger anterior hippocampi than non-taxidrivers (Maguire et al., 2000). This evidence suggests that spatial experience can increase hippocampal volume. The brains measured by Sherry et al. (1996) came from laboratory-bred gerbils, presumably with little spatial experience. However, it is unclear why spatial training should increase the hippocampus size of female gerbils more than that of males.

Male rats and mice have wider DG-GCLs than conspecific females (Roof \& Havens, 1992; Tabibnia et al., 1999). The same is true when DG-GCL widths of male and female meadow voles in breeding condition are compared (Galea et al., 1999). In all of these cases the males also tend to be better at spatial tasks than conspecific females (e.g. Mishima et al., 1986; Gaulin \& Fitzgerald, 1989; Seymoure et al., 1996; Kavaliers et al., 1998; Cimadevilla et al., 1999). There were no sex differences in DG-GCL width in gerbils. It is possible that the poor quality of the sections obscured any sex differences, but as there were no sex differences in spatial ability in these gerbils there was no a priori reason to expect the males to have a wider DG-GCL than the females.

There were three correlations between spatial ability and hippocampus parameters:

1) Males, but not females, with wider DG-GCLs made more working memory errors on the reference RAM task. 
2) Females, but not males, with larger right hippocampal volumes spent more time in the correct quadrant during the MWM probe trial.

3) In both sexes, individuals with wider DG-GCLs took longer to find the hidden platform during MWM training. This effect is in exactly the opposite direction to that found by Roof and Havens (1992) in rats.

As none of the p-values in the regression analysis were significant after the Bonferroni adjustment I am wary of attaching too much significance to these results, especially as they were not consistent between measures of spatial ability and did not tend to be in the predicted direction or apply to both sexes.

Hemispheric functional asymmetry, that is different functions for equivalent regions of the right and left brain hemispheres, is widely accepted in humans (e.g. Kimura, 1999). The fact that males tend to have more asymmetrical brain functions than females is an explanation for sex differences in spatial ability in humans (section 1.3.1.2). This functional asymmetry may be related to the morphological asymmetry seen in both rodents and humans, which tends to be greater in males than females (Diamond, 1987; Diamond, 1991; Juraska, 1991). In male rats, mice and voles the right DG-GCL is larger than the left DG-GCL, but this difference is not seen in females of these species (Roof \& Havens, 1992; Galea et al, 1999; Tabibnia et al., 1999). Sex differences in hemispheric asymmetry were not found in the gerbils in this study, although there was a non-significant trend for the left DG-GCL to be wider than the right in both sexes.

\subsection{General discussion and future work}

I did not find any sex differences in hippocampus morphology or spatial ability in Mongolian gerbils. My results are counter to Sherry et al.'s (1996) report of sex differences in hippocampal volume in this species, and the prediction from the range size hypothesis that there should be sex differences in spatial ability. There are a number of possible explanations for this:

1. The gerbils were not in breeding condition. 
This explanation has already been partially discussed in section 6.6.3. As well as sex differences in hippocampal volume being found only during the breeding season in meadow voles, sex differences in range size and spatial ability are also only apparent during the breeding season in both meadow voles and deer mice (Gaulin \& Fitzgerald, 1986; Galea et al., 1994a; 1994b; Galea et al., 1995; Kavaliers et al., 1998). If the gerbils in this study were not in breeding condition this could explain why I did not find any sex differences in spatial ability or hippocampal volume. The gerbils in Agren et al.'s (1989) range size study were reproductively active when sex differences in range size were recorded. However, as I have already stated, I have no reason to believe that the gerbils used in this study were not in reproductive condition. Gerbils are sexually mature at 12 weeks of age (B\&K Universal Group Catalogue; Norris, 1987) and the gerbils used in this study were 12 weeks old when' they first arrived in the animal house, they were tested in the RAM several weeks later.

\section{Artificial selection has eliminated sex differences.}

Domestication may also have affected gerbil spatial ability. Gerbils have been bred in captivity since 1935 (Norris, 1987). During this time they will have been exposed to numerous artificial selection pressures that are likely to have significantly altered their behaviour from that of wild gerbils, for example selection for easy breeding under laboratory conditions and for docility. Domestication can also affect brain morphology: it has reduced the size of various brain regions, including the hippocampus, in geese and pigs (Kruska \& Röhrs, 1974; Ebinger \& Löhmer, 1987). Sampling error may also have had an influence, as all domesticated gerbils are derived from only 20 pairs taken from a single population (Norris, 1987). However, Sherry et al. (1996) found sex differences in hippocampus size in domesticated gerbils.

There may be differences in the behaviour and brain morphology of different strains of domesticated gerbils. Large differences in behaviour and brain morphology are often observed when different strains of laboratory rats and mice are compared (e.g. Andrews, 1996; Ramos et al., 1997; Hyde et al., 1998; Tabibnia et al., 1999; 
Faraday, 2002). Therefore, it is possible that sex differences in hippocampal volume and spatial ability may be found if a different strain of domesticated gerbils was tested.

\section{Lack of sex-specific spatial experience.}

It is possible that the sex differences in spatial ability observed in meadow voles by Gaulin and Fitzgerald $(1986 ; 1989)$ were due to their wild-caught males and females having had different spatial experiences. Ranging over larger areas could cause the development of better spatial ability. However, there is little evidence that it is the cause of the observed sex differences in spatial ability as sex differences in spatial ability have been observed in captive-bred rodents that have not had variation in spatial experience (e.g. Mishima et al., 1986; Galea et al., 1994a; Seymoure et al., 1996; Cimadevilla et al., 1999; LaBuda et al., 2002). A lack of sex differences in experience seems to be an unlikely explanation as to why the gerbils in this study did not exhibit sex differences in spatial ability.

As I have already discussed (section 6.6.6), spatial experience can affect hippocampus size and therefore sex-specific spatial experiences may be necessary for sex differences in hippocampus size (Clayton \& Krebs, 1994; Maguire et al., 2000). However, Sherry et al. (1996) found sex differences in hippocampus size in a sample of laboratory-bred gerbils. Therefore, the lack of sex-specific spatial experience is as unlikely an explanation as to why I did not find sex differences in hippocampus size, as it is as to why I did not find sex differences in spatial ability.

4. There are no sex differences in spatial ability, range size or hippocampal volume in gerbils.

The sex difference in gerbil range size may not be biologically significant, and therefore not have had an effect on the evolution of spatial ability. As I have already discussed in section 6.4.3 it may be that the difference in range size between male and female gerbils is not very large, or there may only be sex differences in range size in the population studied by Agren et al. (1989), and not in the species as a whole. The population used in this study and that tested by Agren et al. (1989) were 
Chapter 6: Sex differences in spatial ability and the hippocampus in gerbils?

not the same. Another concern is that the evidence for sex differences in range size and hippocampal volume in gerbils come from single papers.

To conclude, it seems unlikely that the reproductive state of my subjects, or their lack of natural spatial experiences could have been the reason why I did not find sex differences in spatial ability or hippocampal volume in gerbils. It is possible that I did not find sex differences because the gerbils that I used were domesticated or because there are differences between gerbil strains as to whether sex differences are found or not. 


\section{Chapter 7: Sex differences in spatial and object memory in humans, and the 'hunter-gatherer' hypothesis.}

\subsection{Introduction}

The 'female foraging' hypothesis complements the 'male foraging' hypothesis as an explanation of the evolution of sex differences in spatial ability in humans (Silverman \& Eals, 1992; chapter 1). The pair of hypotheses state that in the hypothetical "environment of evolutionary adaptedness" humans had a division of foraging labour between the sexes, so that men hunted for animals while women foraged for stationary food items such as plants. This leads to the prediction that men and women will have evolved to excel at the spatial tasks involved in each of their foraging styles. The female foraging hypothesis states that women should be better than men at tasks involved in locating food sources embedded within complex arrays of vegetation and finding them in ensuing growing seasons (Silverman \& Eals, 1992; Eals \& Silverman, 1994).

The female foraging hypothesis emphasises the spatial nature of gathering, describing gatherers as needing:

"the capacity to rapidly learn and remember the contents of object arrays, and the spatial relationships of the objects to one another" (Silverman \& Eals, 1992; my emphasis)

Eals and Silverman also stress that the male spatial superiority is not universal, and that women should excel at some spatial tasks:

"if the disposition for the development of specific spatial attributes evolved in the male in conjunction with hunting, females may have developed parallel spatial specializations associated with foraging." (Eals \& Silverman, 1994)

The female foraging hypothesis has been tested using object-location tasks. 
The first test of the female foraging hypothesis involved a pencil-and-paper task (Silverman \& Eals, 1992). Subjects were given a 'stimulus array', which consisted of a piece of paper showing pictures of familiar objects such as a guitar, bird and teapot. After the subjects had examined the objects for $1 \mathrm{~min}$ the stimulus array was put away. Subjects were then given a copy of the 'response array'. This contained the same objects as the stimulus array, but some of them had exchanged locations. Subjects were asked to mark items that were not in their original location. Women performed better than men on this task.

Since this original test women have been found to score higher than men on several other object-location tasks. Women remembered the locations of common objects, such as chalk and shoes, in a real room more accurately than men (Montello et al., 1999). Women have also been shown to perform better than men at the card game Memory ${ }^{\mathrm{TM}}$ (McBurney et al., 1997). This game involves a set of cards with pictures of objects on them, each picture appears on two cards. The cards are randomly placed facedown on a flat surface. The player is allowed to turn over any two cards; when two identical cards have been turned over they are removed from the game, otherwise they are turned facedown again. The object of the game is to find all of the pairs of cards in the minimum number of turns.

Women also score higher than men on an object-location task similar to the Memory game, except that flaps are lifted rather than cards turned in order to reveal the objects. Women scored higher irrespective of whether the objects to be remembered were coloured dots or line drawings of geometric forms such as circles, squares and crosses (Duff \& Hampson, 2001).

However, object-location tasks are not entirely spatial; rather they involve two components. The subjects have to remember both what the objects were (object or feature memory) and where they are located (spatial location memory). In objectlocation tasks that emphasise the location memory component more, women no longer outperform men. In Silverman and Eals' original pencil-and-paper objectlocation task, the moved objects in the response array had simply exchanged locations and the pattern of empty space was the same as in the stimulus array 
(Silverman \& Eals, 1992). When the task was made more spatial by moving some objects in the response array to locations that were previously unoccupied, women no longer outperformed men (James \& Kimura, 1997). Similarly, there were no differences between men and women's performance on an object-location task where the subject was given pictures of the objects present in the stimulus array and had to replace them in the correct locations (Postma et al., 1998).

These results suggest that it is the object memory part of the object-location task, rather than the location component, at which women excel. Women tend to be better than men at remembering objects (e.g. Silverman \& Eals, 1992; Galea \& Kimura, 1993; Chipman \& Kimura, 1998; Duff \& Hampson, 2001). The location component of object-location tasks is the truly spatial part, and therefore these studies suggest that women may never have better spatial ability than men, even on object-location tasks.

I developed two simple memory tasks that were similar to each other in all respects except that one could only be solved using location cues, while the other could only be solved using visual features. I predicted that if the female foraging hypothesis were correct, then women would perform better than men on both tasks. As an alternative hypothesis I predicted that women would perform better than men on the feature task, but the well-documented male advantage in spatial ability would result in the men performing better than the women on the location task.

\subsection{Pilot study}

\subsubsection{Materials and methods}

The tasks were developed from a computer program previously used to test the feature and location memory of songbirds (Parus spp.). Earlier work had suggested that it might also be suitable for testing humans. The version of the program that $I$ used utilised a normal computer mouse as the input device, rather than the touchscreen that the birds used to interact with the program. Unlike the birds, the human subjects did not get a reward for a correct choice, but they were told when they got a trial correct. 
The program was written in BBC Basic and run on an Acorn emulator (Red Squirrel 0.4.4) on a Windows PC. The feature and location versions of the program were similar except that the feature task could only be solved using feature cues, while the location task could only be solved with location cues (figure 7.1).

Figure 7.1: Diagrammatic representation of a single trial of each of the tasks used in the pilot study. The coloured squares in the feature task represent photographs. After an inter-trial interval of $5 \mathrm{sec}$ the trial was repeated using randomly generated locations and pictures.

Location task
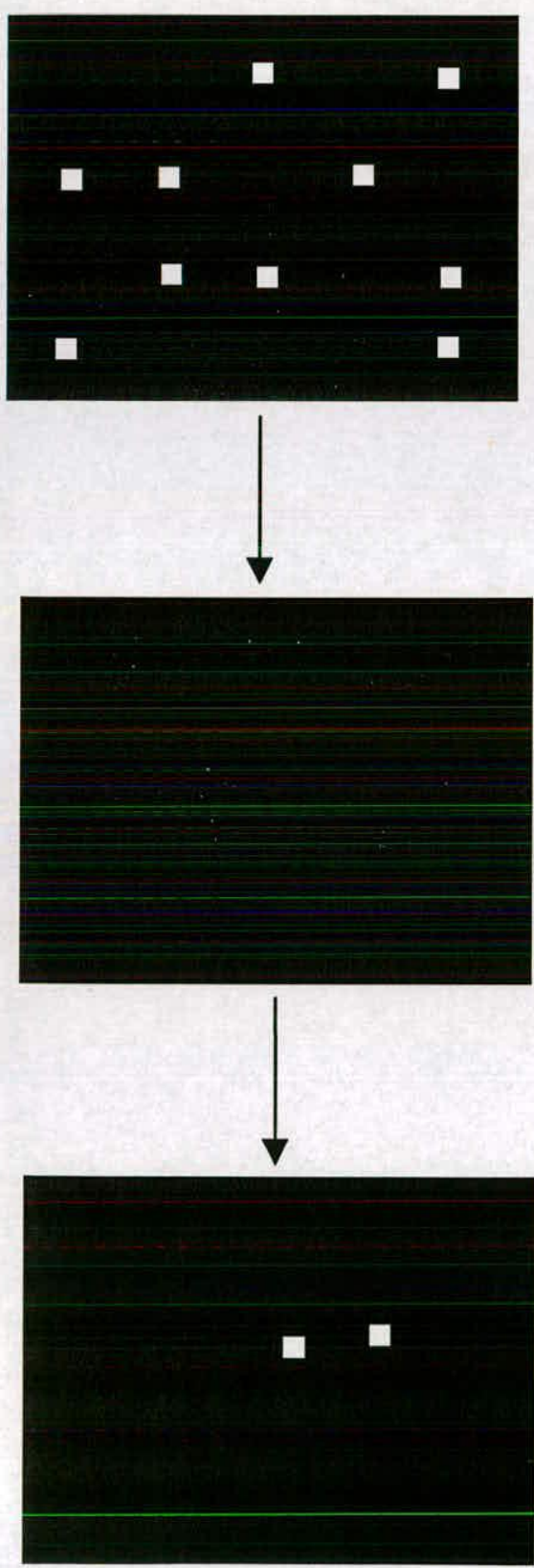

Feature task

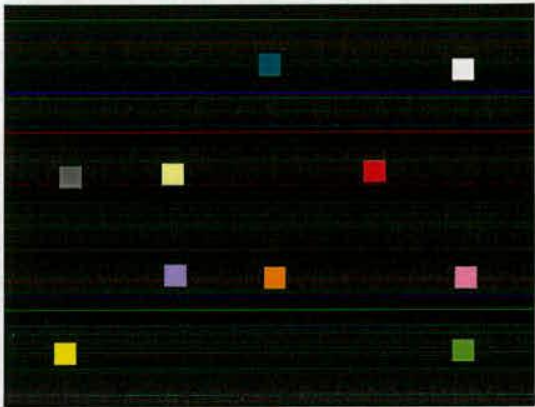

Retention interval $20 \mathrm{sec}$

Choice phase 
The initial sample phase of both tasks consisted of ten square items displayed in random locations in a $5 \times 4$ grid on the computer screen for $10 \mathrm{sec}$. There was then a retention interval of $20 \mathrm{sec}$ during which the screen was blank except for ' $\mathrm{RI}$ ' followed by the trial number in the top left corner. After the retention interval had elapsed the subject was presented with two items, one of which was the same as one previously seen and the other different from those previously seen (choice phase). The subject had to use the mouse to click on the different item. If they chose the different item the word "correct" was briefly displayed on the screen. There was a $5 \mathrm{sec}$ inter-trial interval, during which the screen was blank except for the phrase "next trial is" followed by the number of the next trial displayed in the top left corner. 20 trials were given of each of the tasks.

The items in the feature task were colour photographs randomly chosen from a selection of 20 man-made and natural objects such as a flower, motorbike, dolphin, starfish and hot-air balloon. Different locations were used for the choice phase than were used in the sample phase, so that location could not be used to solve the feature trials. In the choice phase the two photographs consisted of one of the original photos and a previously unseen photo of a similar object.

The items in the location task were all plain white squares. In the choice phase one square appeared in a location previously occupied during the sample phase, while the other square appeared in a previously unoccupied location that was adjacent to the other square.

At the end of the 20 trials the program saved information about the choices made and the speed of response as a spreadsheet file. While the subject was doing the task the experimenter sat quietly out of the subject's sight. Testing took approximately $30 \mathrm{~min}$ per subject. The two tasks were given to the subjects in a pseudorandom order so that approximately half of the males and half of the females received the feature task first.

Data were collected from 12-28 February 2002, between 1200 and 1730 . Two experimenters (A \& B) collected the data using separate rooms and computer 
equipment. The subjects were individually instructed in how to use the program immediately prior to testing. The subjects were all young adults who were studying for, or had recently completed, undergraduate degrees. Subjects who did not complete 20 trials on both the feature and location tasks, or who had missing data, were excluded from the analysis. One subject persistently chose the choice item that he had seen before rather than the new one, his data were excluded from the analysis. Data from 53 subjects (10 males and 13 females tested by experimenter A, and 12 males and 18 females tested by experimenter $B$ ) were analysed.

\subsubsection{Results and analysis}

The data were analysed using the general linear model (GLM) equivalent of a repeated-measures analysis of variance (ANOVA) with three between-subjects factors (sex, order and experimenter) and one within-subject factor (task). The following model was used:

Score $=$ sex + order + experimenter + sex*order + sex*experimenter + order*experimenter + sex*order*experimenter + subject(sex order experimenter $)+$ task + order*task

None of other interactions involving task were significant, and so they were excluded from the model. The between-subjects interactions could not be removed from the model because subject was nested within them. The F-ratios for the between subject effects were calculated using the 'subject(sex order experimenter)' mean square (MS) as the denominator. The untransformed data met the parametric assumptions of homogeneity of variance and normality of error.

The only significant effect was the order by task interaction $\left(\mathrm{F}_{1,51}=14.11, \mathrm{p}<0.001\right.$; figure 7.2.a). Subjects scored higher on the location task when they did the feature task first (Tukey simultaneous test $\mathrm{t}=-3.252, \mathrm{p}=0.0106$ ). Subjects tended to score higher on the feature task when they did the location task first, but this effect was not statistically significant (Tukey simultaneous test $\mathrm{t}=1.9811, \mathrm{p}=0.2085$ ). Neither the main effect of experimenter nor any of its interactions were significant $\left(F_{1,45}=0.38\right.$, $\mathrm{p}=0.539$ ). Neither the main effect of sex nor any of its interactions were significant 
$\left(\mathrm{F}_{1,45}=0.32, \mathrm{p}=0.577\right)$. However, the trends for the sex by task interaction were in the predicted direction with men tending to perform better on the location task than women, and women tending to perform better on the feature task than men (figure $7.2 . b)$.

Figure 7.2: Mean scores on the feature and location tasks during the pilot study. Error bars show standard error of the mean (SEM).

a) Effect of task order. Feature first $n=27$, location first $n=26$.

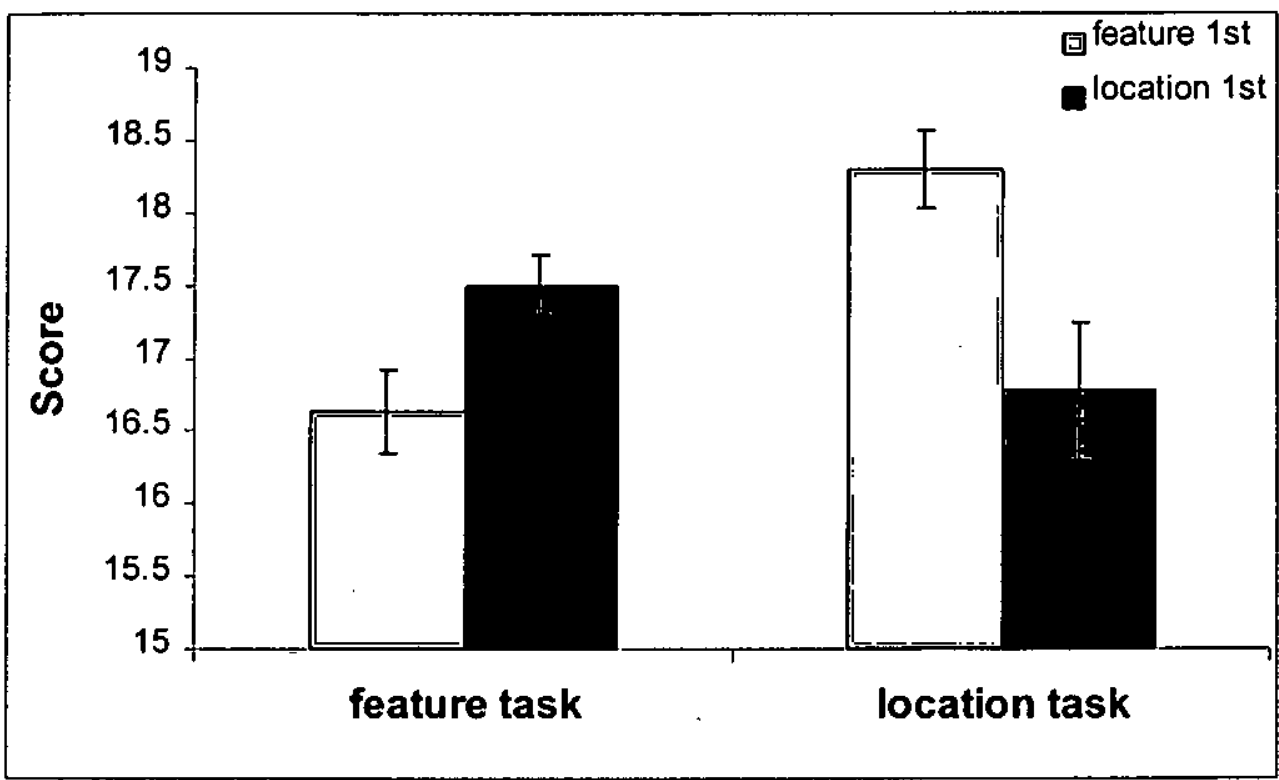


b) Effect of sex. Men $n=22$, women $n=31$.

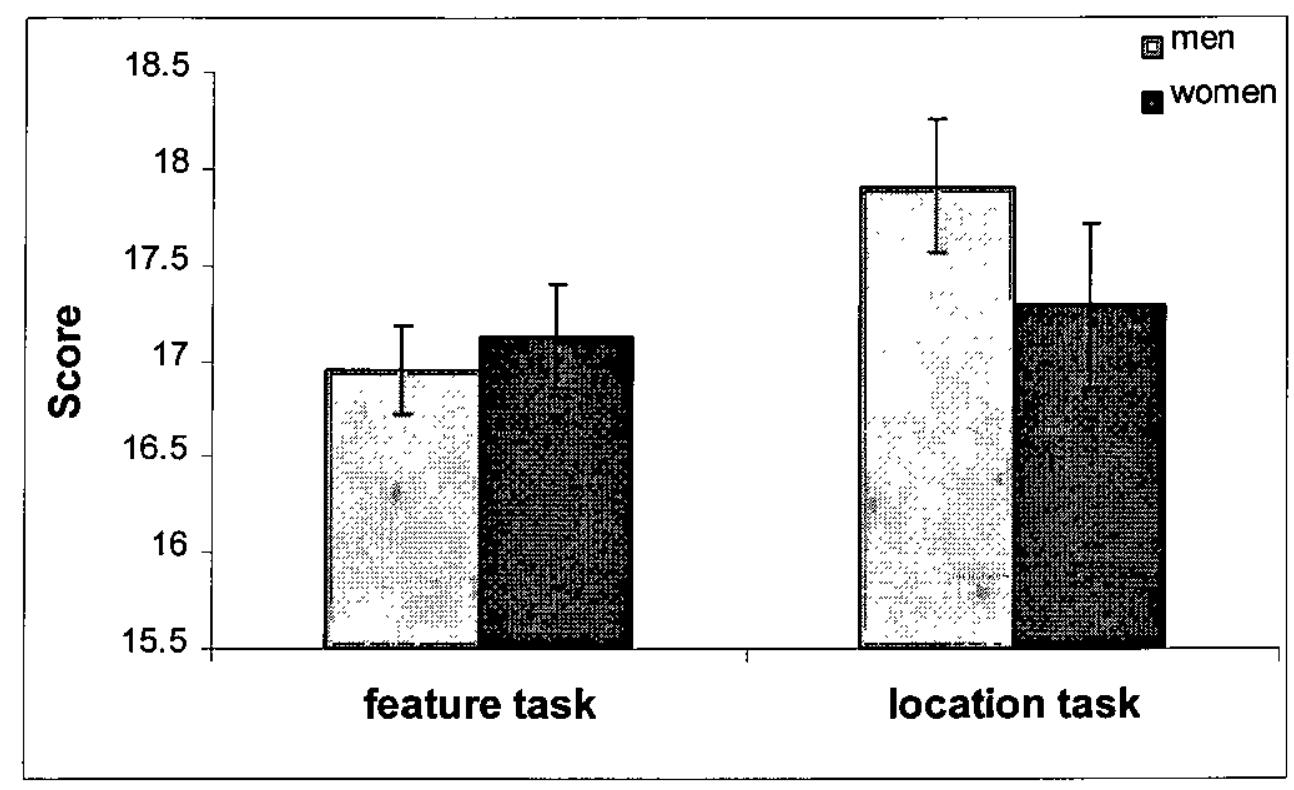

\subsubsection{Discussion}

There were no differences between the scores of subjects tested by the two experimenters. This suggests that any differences in the methods or the subjects used by the two experimenters did not affect the subjects' scores.

Subjects achieved higher scores on the second task irrespective of whether this was the feature or location task. This may have been because the subjects acquired some general knowledge about how to do the tasks. This effect could possibly have been removed by allowing the subjects to try out the task before actually testing them on it. However, this effect was controlled for in both the experimental design and analysis and so I decided not to introduce a training session, as this would have increased the amount of time that it took to test the subjects.

As predicted men tended to score higher on the location task than women and the opposite was true of the feature task scores. The subjects mentioned a couple of problems that may have affected the scores on the tasks. Subjects reported that they did not have time to look at all ten of the sample pictures in the feature task during 
the $10 \mathrm{sec}$ allowed. Some subjects also mentioned that they remembered the locations on the location task as a pattern on the screen rather than as individual items, this could have made the task less spatial and made it easier for the women. Both of these problems were addressed by changing the program before continuing testing.

\subsection{Main experiment}

\subsubsection{Materials and methods}

The two tasks were similar to those used in the pilot study, except that instead of getting $10 \mathrm{sec}$ to view the items before the screen went blank for the retention interval, the subjects used the computer's mouse to click on each item. When an item was clicked on, it disappeared and when all of the items had disappeared the retention interval started. The intention was to allow the subjects time to view each of the pictures in the feature task. It might also have made the subjects pay more attention to the location of individual squares in the location task rather than paying attention to the overall pattern that they formed. It is possible that this "point and click" method may have affected the sexes' performance differentially if the fact that the stimuli were disappearing one by one, and so were there for different amounts of time and could not be learned as an array, favoured one kind of memory over another and affected males and females differently. I have been unable to find any evidence for this in published studies, but it may be worth trying to test for such an effect in the future (for example, a study could be designed to test whether presenting the stimuli sequentially, rather than simultaneously as a complete array, affects the scores obtained by men and women on the two tasks).

The subjects took approximately $40 \mathrm{~min}$ to complete both tasks. I also introduced a short questionnaire that was completed before testing and recorded the subject's age, degree subject and year of study or number of years since graduating. All subjects were tested between 1100 and 1800 .

Apart from the changes described above, the methods used were the same as those in the pilot study. A total of 47 subjects were tested, none of whom took part in the 
pilot study. Experimenter A tested 18 subjects ( 9 men and 9 women) from 5 March 25 April 2002. Experimenter B tested 29 subjects (12 men and 17 women) on 6 and 7 March 2002.

\subsubsection{Results and analysis}

The same repeated-measures ANOVA was used to analyse the data as was used in the pilot experiment, except that the experimenter by task and sex by task interactions were significant and so were retained in the final model, while the order by task interaction was not. The data were square-root transformed in order to meet parametric test assumptions.

The experimenter by task interaction was significant $\left(\mathrm{F}_{1,44}=7.31, \mathrm{p}=0.010\right.$; figure 7.3.a). The subjects tested by experimenter $B$ performed significantly higher on the feature task than those tested by experimenter A (Tukey simultaneous test $t=3.408$, $\mathrm{p}=0.0074$ ). There was no significant difference between the location scores of the subjects tested by the two experimenters (Tukey simultaneous test $\mathfrak{t}=-0.3986$, $\mathrm{p}=0.9783$ ).

The sex by task interaction was significant $\left(\mathrm{F}_{1,44}=16.13, \mathrm{p}<0.001\right.$; figure 7.3.b). This was largely due to the men scoring significantly higher on the location task than the women (Tukey simultaneous test $\mathrm{t}=4.164, \mathrm{p}=0.0005$ ). The women tended to score higher than men on the feature task, but this effect was not statistically significant (Tukey simultaneous test $\mathrm{t}=-1.111, \mathrm{p}=0.6845$ ). 
Figure 7.3: Mean scores on the feature and location tasks during the main experiment $+/$ - SEM.

a) Effect of experimenter. Experimenter $A n=18$, experimenter $B n=29$.

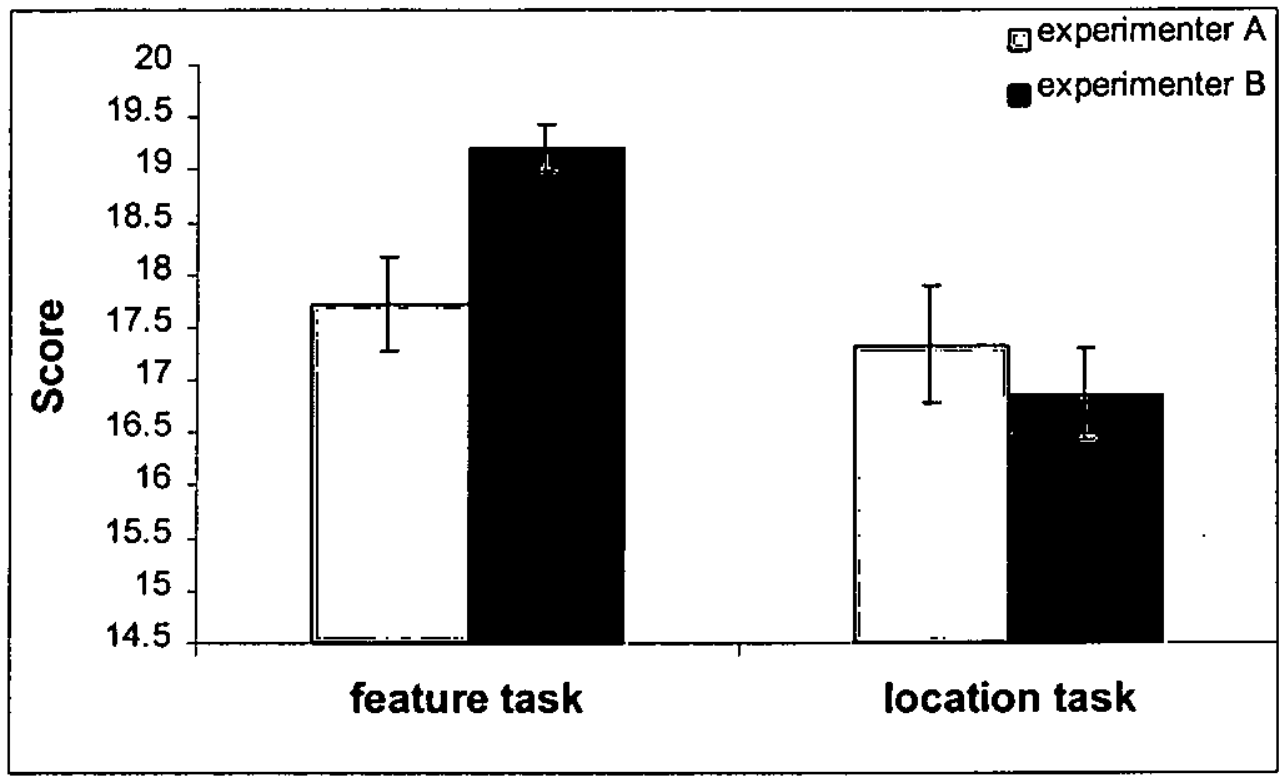

b) Effect of sex. Men $n=21$, women $n=26$.

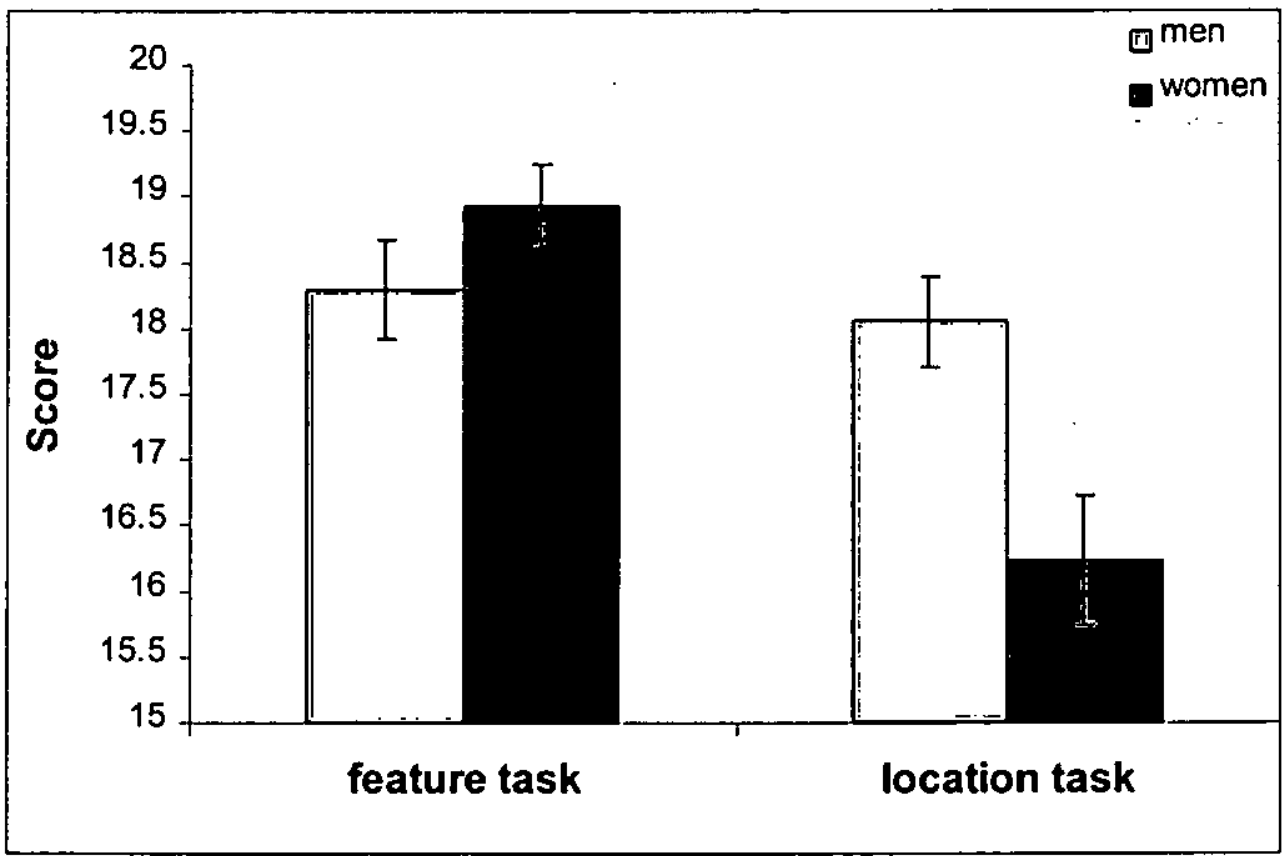


The mean age of the subjects was 21.81 years (range 20 to 24). The data were checked to see if there were any differences in age between the sexes or the subjects tested by each experimenter, using one-way ANOVAs. The ages of the men and women were not significantly different $\left(\mathrm{F}_{1,45}=2.94, \mathrm{p}=0.093\right)$, although the men did tend to be older than the women (men: mean 22.14, range 20 to 24; women: mean 21.54 , range 20 to 24 ). The two experimenters tested subjects of significantly different ages $\left(\mathrm{F}_{1,45}=9.24, \mathrm{p}=0.004\right)$. The subjects tested by experimenter $\mathrm{A}$ were older than the subjects tested by experimenter B (A mean 22.44, range 21 to 24; B mean 21.41 , range 20 to 24 ).

In order to test whether age affected score I fitted the GLM 'score = age + sex + experimenter', with age as a covariate, separately to the feature and location scores. This tested whether there was a significant effect of age on score within the four groups (sexes and experimenters). The effect of age was not significant for either feature or location scores (figure 7.4 ; feature: $F_{1,43}=1.36, p=0.249$; location: $\mathrm{F}_{1,43}=0.45, \mathrm{p}=0.504$ ). Therefore, the observed differences in spatial ability were unlikely to be due to differences in the age of the subjects in each group.

Figure 7.4: Regression plots of score on age, by group.

a) Location scores.

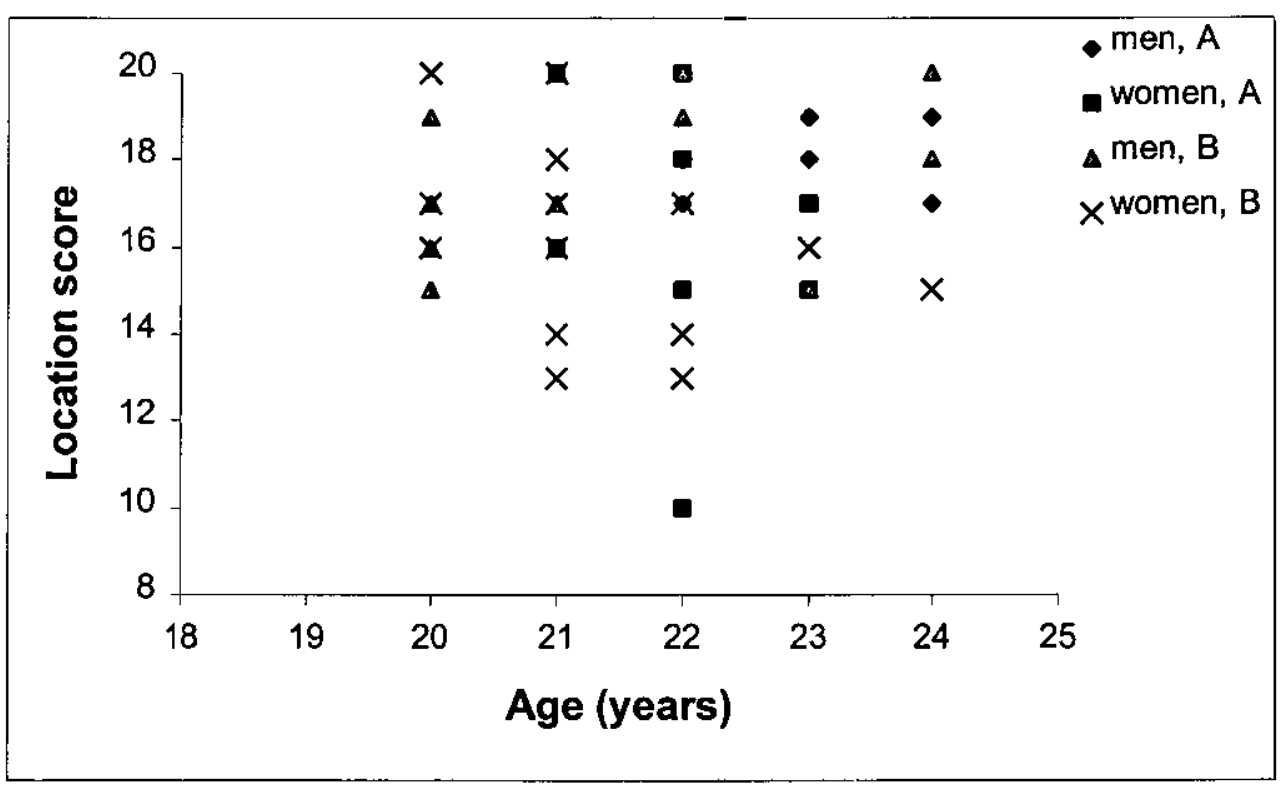


b) Feature scores.

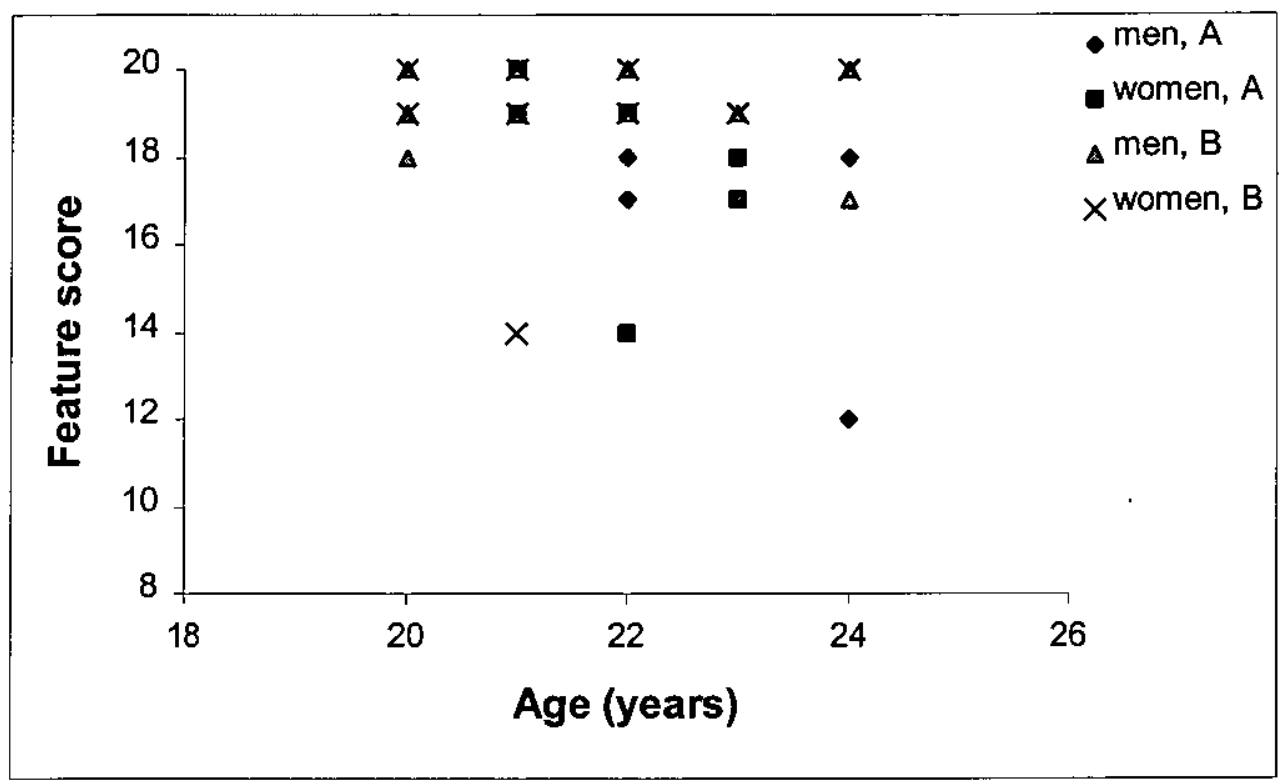

\subsection{Discussion}

The men performed better than the women on the location task and the women tended to perform better than men on the feature task. These differences were not explained by differences in the ages of the men and women tested. The sex difference may only have been significant for the location task because all of the subjects scored highly on the feature task and therefore a difference between the sexes may have been obscured by a ceiling effect. There are several ways in which the task could be made more difficult in order to determine whether this was the case:

1. Extend the retention interval. However, when this was tried during initial calibration of the tasks, subjects complained of boredom and having to spend too much time on the tasks.

2. Increase interference levels by inserting more objects into either the sample or the choice phase. 
3. Introduce a distracter task into the retention interval. This is likely to make it more difficult for the subjects to remember the objects in the sample phase. Initial studies suggested that when a calculation distracter task is used, subjects show less than perfect performance with only four samples. However, it is unclear what sort of task would make an appropriate distracter. Using a task that is known to display sex differences in performance, such as mental rotation, could bias the results if one of the sexes finds it more difficult and is therefore more distracted by it. Also, distracter tasks that involve feature or location memory may interfere more with one of the tasks than the other.

The effect of experience on performance seen in the pilot study was not present in the main experiment. The learning effect in the pilot study may have been due to the subjects having to learn to remember all of the objects within the 10sec allowed in the sample phase. In the task used in the main experiment the subjects had as long as they wanted to view the samples before going on to the retention interval and the choice phase.

There were differences in the scores obtained from subjects tested by the two experimenters. Subjects tested by experimenter B scored higher than those tested by experimenter A, but only on the feature task. This difference was not present in the pilot study. It is not explained by age differences between the subjects tested by each experimenter, but there may have been other differences between the subjects, such as in general intelligence, that could explain this difference. It is also possible that the experimenters' methods differed. It is not clear why this effect is only seen on the feature task.

The finding that men outperform women on a spatial location task is in the opposite direction to the predictions made by the female foraging hypothesis, and suggests that men are better than women at all spatial tasks, while women are better than men at remembering features. At the very least, women need some sort of feature information in order to excel at object-location tasks. I propose that the female foraging hypothesis is still a valid explanation of women's superior feature memory, 
and therefore better ability at object-location tasks, but the spatial component of the hypothesis is not consistent with the data.

My findings, and the male and female foraging hypotheses, can be integrated with the observation that the sexes tend to solve spatial tasks in different ways (sexspecific cognitive styles, section 1.3.4). Women use proximate landmark cues, while men rely on distant abstract cues such as geometry and compass direction (e.g. Galea \& Kimura, 1993; Dabbs et al., 1998; Sandstrom et al., 1998; Montello et al., 1999; Saucier et al., 2002). Women may utilise landmark cues because they are good at remembering features, while men use the more truly spatial abstract cues. These cognitive styles may relate to the foraging hypotheses if the sex-specific approach is the best way of solving the relevant foraging task. The spatial method may be the most effective way of solving navigation tasks, resulting in men being better at navigation than women, while the object/landmark method may be the more efficient way of solving object-location tasks, causing women to excel at these tasks.

Further work on this topic should include giving men and women an object-location task to see whether there is a sex difference on such a task when given on the computer. If a sex difference is not found, then it would be worth manipulating the task to see whether small changes may result in a sex difference. Manipulating the relative value of feature and location cues in solving the task may make it easier for one sex to solve than the other. If the task relies heavily on feature cues women may be better at solving it than men. The feature and location cues could be manipulated in several ways, for example by making the different features and/or locations more similar and therefore harder to distinguish; by using location switches rather than new locations in the choice phase; or by using unfamiliar items may be more difficult to remember because verbal labels are not readily available.

If a sex difference is found on the object-location task, the components of the task could be 'unpacked' in order to find out where the sex difference occurs. This could involve testing whether there are sex differences in object memory, location memory, or the ability to combine the two ('object in place' memory). Men and women could be tested to see whether they use different cues to solve object-location tasks. The 
tasks used in this chapter could be combined into an object-location task with choice phase choices between a previously seen picture in the same location as in the sample phase, and a new picture in a new location (the correct choice). I could test whether the sexes use different cues to solve the task by introducing probe trials. On a probe trial the choice phase choices would be between a previously-seen picture in a new location and a new picture in a previously-occupied location. I would expect the men to choose the new location and the women to choose the new picture as being the different and therefore correct choice. This could also be tested with laboratory rats, as these animals have also been shown to use sexually dimorphic strategies to solve navigation tasks (Williams et al., 1990; Kanit et al., 1998a; Roof \& Stein, 1999; Tropp \& Marcus, 2001b). 


\section{Chapter 8: General discussion}

\subsection{Sex differences in spatial ability in laboratory rats}

In chapter 2 I gave laboratory rats two commonly used maze tests (the radial arm maze and the Morris water maze), and was unable to find sex differences in spatial ability using either task. After these experiments I decided to concentrate on external factors that might cause in sex differences in spatial ability in laboratory rats. However, it is still possible that there are sex differences in spatial ability in laboratory rats and that by manipulating the tasks I could have found the exact conditions under which these occur. For example, I could have made the tasks more difficult by increasing the number and/or length of arms in the RAM, I could also have changed the training schedule, the type of cues available, or tried using different strains. If a spatial task could be found on which male lab rats consistently outperform females this would have provided a baseline from which I could have made changes to see how the sex differences were affected. This would have been almost the opposite approach to the one used in this thesis, where I used a task with no sex differences and manipulated the subjects to see if sex differences would develop.

I have been unable to find a sex difference in spatial ability in laboratory rats, or to conclusively prove that the observed sex differences can be caused by experimental factors. However, my research has gone some way towards defining the question, and has produced four possible explanations that may be productive areas for future work:

\section{Uneaten reward errors}

Differences in the frequencies with which males and females made uneaten reward errors (entering a rewarded arm but failing to eat the reward) resulted in an apparent sex difference in spatial ability in the radial arm maze experiment in chapter 2. This 
could explain the sex differences in spatial ability under specific circumstances (i.e. if a study used a particular correct arm entry criterion in the radial arm maze). Further experiments (chapters $4 \& 5$ ) suggested that stress and food restriction regime did not influence these errors. I hypothesised that they were due to higher exploration motivation in female than in male rats, but this is presently untested.

\section{Oestrous cycle effects}

I was unable to find any effect of oestrous cycle stage on female rats' recall of a reference memory water maze task (chapter 3 ), however my experiment suffered from low power and so I was unable to satisfactorily conclude that there was no effect. As there is already some evidence for an oestrous cycle effect on spatial ability working memory tasks (Frye, 1994; Warren \& Juraska, 1997) I think that it would be useful to repeat this experiment with a larger sample size. This would allow me to test whether the oestrous cycle could also be a possible cause of sex differences in reference memory spatial tasks.

\section{Stress}

$15 \mathrm{~min}$ of restraint stress immediately prior to testing in the radial maze did not differently affect males and females, although it did improve both sexes' working memory. I had hypothesised that female rats were more stressed than males by the novelty of maze testing and that this reduced the females' spatial ability. However, using restraint stress to apply additional stress to the experimental group may not have been an appropriate way to test this hypothesis. I assumed that different types of stress would have similar, additive, effects (e.g. Stillman et al., 1998), but it is possible that this is not the case and that acute restraint stress is not equivalent to stress caused by a novel task. Another way to test my hypothesis would be to experimentally manipulate the novelty of the task and see if this affected males and females differently.

\section{Food restriction}


I found that level of food restriction did differentially affect the sexes' performance in the radial maze. Female rats displayed impaired reference memory when maintained at $85 \%$ of their free-feeding body-weight, compared to females maintained at $95 \%$ and males maintained at $85 \%$ of their free-feeding body-weight. This result was in the opposite direction to that predicted, and suggests that food restriction may directly affect spatial cognition in female rats. I would like to carry out further experiments in order to see whether this result is replicable, whether it can be found using different spatial tests, and to determine why it is only present in females.

The explanation for an inconsistently found sex difference in spatial ability in laboratory rats does not appear to be simple. It is likely that there are multiple factors that influence whether or not a sex difference is found, including the above, as well as effects such as organisational hormone levels, the amount of pretraining given, and the age and strain of the subjects.

A general problem with the RAM tasks was that reference memory errors were generally high, raising the question of how well the task had been learnt. Learning of the task may be more clearly demonstrated by using the number of correct entries in the first four visits as a measure of spatial ability (as in chapter 6). The reference memory task may not have been learnt because there was not enough incentive for the subjects to learn it, i.e. there was not a significant cost involved in checking the arms for rewards and so it was easier for the subjects to check every arm for rewards rather than remember which arms contained rewards. The RAM arms could have been made longer, or doors put on the arms so that the subjects would have to push them open. Either of these alterations would mean that the subjects experienced a higher cost for entering an unrewarded arm, and might encourage then to learn which arms contain rewards. Using a more palatable reward might have a similar effect.

\subsection{Sex differences in spatial ability in Mongolian gerbils}

I was unable to find sex differences in spatial ability in Mongolian gerbils, despite the 'range size' hypothesis predicting that males should be better at spatial tasks than 
females. I was also unable to find sex differences in hippocampal volume, this was in agreement with the results of my spatial tests, but conflicted with the findings of an earlier study (Sherry et al., 1996). These results may be due to domestication effects. The ideal way to test whether Mongolian gerbils have sex differences in range size, hippocampal volume and spatial ability would be to travel to Mongolia and measure the range size of gerbils in breeding condition in the wild. The same individuals could then be captured and given a spatial ability test before being sacrificed and having their hippocampal volumes measured.

\subsection{Sex differences in spatial ability in humans}

From the results of location and feature memory tasks given to human volunteers I concluded that it is likely that women's observed superiority on object-location tasks is due to superior object, rather than spatial, memory. This could be tested by looking at which cues men and women pay attention to when solving object-location tasks, something that I plan to do in the near future. If women are found to rely on their superior memory for features to solve object-location tasks the 'female foraging' hypothesis can be discounted as a possible explanation for the evolution of sex differences in spatial ability.

\subsection{Wider implications}

As well as the specific results discussed above, I think that the work in this thesis has several general implications for the study of sex differences in spatial ability:

One question that is raised is whether spatial ability is too broad a concept to be of use. Although in this thesis I have equated spatial ability with navigational ability, even navigational ability encompasses a wide range of different ways that an animal might use to find its way around the environment. It is possible that researchers should instead focus on components of spatial ability, as I attempted to do in chapters 2 and 7. Components of spatial ability such as memory for landmarks, ability to use compass directions, or recall of response chains, could be tested to see whether they 
vary between sexes. Evolutionary hypotheses could also be reformulated in these terms.

Another implication from this thesis is that studies of sex differences in spatial ability should be careful to choose spatial tests and study species that are appropriate to the question being investigated. For example, a highly domesticated species such as the laboratory rat and an artificial spatial test such as a radial arm maze, may not be the best way to test an evolutionary theory; an appropriate wild species and more natural spatial task might be better, depending on the circumstances. As I have already discussed, the partially-rewarded radial arm maze task that I used may not have been a very good test of spatial reference memory, and a different or modified task should be used in the future. Similarly, the water maze may not have been an appropriate test for the desert-living Mongolian gerbils.

In chapter 1 I analysed the evolutionary hypotheses that have been proposed to explain sex differences in spatial ability. I would like to reiterate that experiments are needed to specifically test and distinguish between the hypotheses that have been proposed. I think that more will be gained from step-by-step work testing current hypotheses, than by adding new untested hypotheses to the confusion. It would be particularly useful to do work to try to distinguish between the four hypotheses ('male foraging', 'range size', male warfare, and 'female choice') that all predict males will have better spatial ability than females because they travel over larger areas, but differ in the reason as to why the males travel further (hunting, polygyny, warfare and displaying to females, respectively). At the moment there is very little data to tell these hypotheses apart, but wild populations of species that vary in these factors could be tested, and this would provide evidence for or against individual hypotheses.

A theme that has recurred throughout this thesis is that of sex differences in cue use. This provides a possible cognitive mechanism for sex differences in spatial ability, and can potentially explain many experimental results. There is evidence that males and females use different cues to solve spatial tasks and this can provide an 
explanation as to why sex differences in rats are inconsistently found, and for the observed sex differences in navigational and object-location tasks in humans.

I think that more work should be carried out in this area, for example:

1. Do any of the evolutionary hypotheses predict sex differences in cue use?

2. In which species do sex differences in cognitive style occur? They have been reported in kangaroo rats (Langley, 1994) as well as in humans and laboratory rats. Can they explain the observed sex differences in spatial ability in meadow voles?

3. What is the mechanism of sex differences in cue use? Is cognitive style affected by gonadal hormones, in the same way as spatial ability is? How is cue use is controlled in the brain? How do cognitive styles develop within an individual, and are they influenced by the environment? 


\section{References}

Agren, G., Zhou, Q., \& Zhong, W. (1989). Ecology and social behaviour of Mongolian gerbils, Meriones unguiculatus, at Xilinhot, Inner Mongolia, China. Animal Behaviour, 37, 11-27.

Amano, M., Hasegawa, M., Hasegawa, T., \& Nabeshima, T. (1993). Characteristics of transient cerebral ischemia-induced deficits on various learning and memory tasks in male Mongolian gerbils. Japanese Journal of Pharmacology, 63, 469477.

Amaral, D. G., \& Witter, M. P. (1989). The three-dimensional organisation of the hippocampal formation: a review of the anatomical data. Neuroscience, 31, 571591.

Anderson, B. J., Rapp, D. N., Baek, D. H., McCloskey, D. P., Coburn-Litvak, P. S., \& Robinson, J. K. (2000). Exercise influences spatial learning in the radial arm maze. Physiology \& Behavior, 70, 425-429.

Andrews, J. S. (1996). Possible confounding influence of strain, age and gender on cognitive performance in rats. Cognitive Brain Research, 3, 251-267.

Andrews, J. S., Jansen, J. H. M., Linders, S., Princen, A., \& Broekkamp, C. L. E. (1995). Performance of four different rat strains in the autoshaping, two-object discrimination, and swim maze test of learning and memory. Physiology \& Behavior, 57(4), 785-790.

Armario, A., Montero, J. L., \& Balasch, J. (1986). Sensitivity of corticosterone and some metabolic variables to graded levels of low intensity stresses in adult male rats. Physiology \& Behavior, 37, 559-561.

Astie, A. A., Kacelnik, A., \& Reboreda, J. C. (1998). Sexual differences in memory in shiny cowbirds. Animal Cognition, 1, 77-82.

Baenninger, M., \& Newcombe, N. (1989). The role of experience in spatial test performance: a meta-analysis. Sex Roles, 20(5/6), 327-344.

Benhamou, S. (1997). On systems of reference involved in spatial memory. Behavioural Processes, 40, 149-163.

Barnea, A., \& Nottebohm, F. (1994). Seasonal recruitment of hippocampal neurons in adult free-ranging black-capped chickadees. Proceedings of the National Academy of Science USA, 91, 11217-11221.

Barnett, S. A., \& McEwan, I. M. (1973). Movements of virgin, pregnant and lactating mice in a residential maze. Physiology \& Behavior, 10, 741-746.

Barrett, R. J., \& Ray, O. S. (1970). Behavior in the open field, Lashley III maze, shuttle-box, and Sidman avoidance as a function of strain, sex, and age. Developmental Psychology, 3(1), 73-77.

Berger-Sweeney, J., Arnold, A., Gabeau, D., \& Mills, J. (1995). Sex differences in learning and memory in mice: effects of sequence of testing and cholonergic blockade. Behavioral Neuroscience, 109(5), 859-873.

Berry, B., McMahan, R., \& Gallagher, M. (1997). Spatial learning and memory at defined points of the estrous cycle: effects on performance of a hippocampaldependent task. Behavioral Neuroscience, 111(2), 267-274.

Beveridge, T. (2001). The effect of stress on the exploratory behaviour and spatial ability of rats. Unpublished Zoology Honours project, Edinburgh University, Edinburgh.

Bilkei-Gorzo, A., Gyertyan, I., \& Levay, G. (1998). mCPP-induced anxiety in the light-dark box in rats - a new method for screening anxiolytic activity.

Psychopharmacology, 136, 291-298. 
Bimonte, H. A., \& Denenberg, V. H. (1999). Estradiol facilitates performance as working memory load increases. Psychoneuroendocrinology, 24, 161-173.

Bimonte, H. A., Hyde, L. A., Hoplight, B. J., \& Denenberg, V. H. (2000). In two species, females exhibit superior working memory and inferior reference memory on the water radial-arm maze. Physiology and Behavior, 70, 311-317.

Bird, R. B., Smith, E. A., \& Bird, D. W. (2001). The hunting handicap: costly signaling in human foraging strategies. Behavioral Ecology and Sociobiology, 50, 9-19.

Boakes, R. A., Boot, B., Clarke, J. V., \& Carver, A. (2000). Comparing albino and hooded Wistar rats of both sexes on a range of behavioral and learning tasks. Psychobiology, 28(3), 339-359.

Boonstra, R., Krebs, C. J., Gaines, M. S., Johnson, M. L., \& Craine, I. T. M. (1987). Natal philopatry and breeding systems in voles (Microtus spp.). Journal of Animal Ecology, 56, 655-673.

Bowman, R. E., Zrull, M. C., \& Luine, V. N. (2001). Chronic restraint stress enhances radial arm maze performance in female rats. Brain Research, 904, 279-289.

Braithwaite, V. A., \& Guilford, T. (1991). Viewing familiar landscapes affects pigeon homing. Proceedings of the Royal Society of London B, 245(1314), 183-186.

Bruderer, B. (1982). Do migrating birds fly along straight lines? In F. Papi \& H. G. Wallraff (Eds.), Avian Navigation (pp. 3-14). Berlin: Springer.

Bryan, J., \& Tiggemann, M. (2001). The effect of weight-loss dieting on cognitive performance and psychological well-being in overweight women. Appetite, 36 , 147-156.

Bucci, D. J., Chiba, A. A., \& Gallagher, M. (1995). Spatial learning in male and female Long-Evans rats. Behavioral Neuroscience, 109(1), 180-183.

Buchner, A., Erdfelder, E., \& Faul, F. (1997). How to use G*Power (WWW document).

Burgess, N., Jeffery, K. J., \& O'Keefe, J. (1999). Integrating hippocampal and parietal functions: a spatial point of view. In N. Burgess, K. J. Jeffery, \& J. O'Keefe (Eds.), The Hippocampal and Parietal Foundations of Spatial Cognition (1st ed., pp. 3-29). Oxford: Oxford University Press.

Buss, D. M., \& Shackelford, T. K. (1997). Human aggression in evolutionary psychological perspective. Clinical Psychology Review, 17(6), 605-619.

Bussiere, L. F. (2002). A model of the interaction between 'good genes' and direct benefits in courtship-feeding animals: when do males of high genetic quality invest less? Philosophical Transactions: Biological Sciences, 357(1419), 309317.

Carney, J. M., Starke-Reed, P. E., Oliver, C. N., Landum, R. W., Cheng, M. S., Wu, J. F., \& Floyd, R. A. (1991). Reversal of age-related increase in brain protein oxidation, decrease in enzyme activity, and loss in temporal and spatial memory by chronic administration of the spin-trapping compound N-tert-butyl- $\alpha$ phenylnitrone. Proceedings of the National Academy of Sciences USA, 88, 36333636.

Chagnon, N. A. (1983). Yanomamo: The Fierce People. (3rd ed.). New York: Holt, Rinehart and Winston.

Chambers, L. K., Singleton, G. R., \& Krebs, C. J. (2000). Movements and social organization of wild house mice (Mus domesticus) in the wheatlands of northwestern Victoria, Australia. Journal of Mammalogy, 81(1), 59-69.

Cherrier, M. M., Asthana, S., Plymate, S., Baker, L., Matsumoto, A. M., Peskind, E., Raskind, M. A., Brodin, K., Bremner, W., Petrova, A., LaTendresse, S., \& Craft, 
S. (2001). Testosterone supplementation improves spatial and verbal memory in healthy older men. Neurology, 57, 80-88.

Chesler, E. J., \& Juraska, J. M. (2000). Acute administration of estrogen and progesterone impairs the acquisition of the spatial Morris water maze in ovariectomized rats. Hormones and Behavior, 38, 234-242.

Chiba, A. A., Kesner, R. P., \& Jackson, P. A. (2002). Two forms of spatial memory: a double dissociation between the parietal cortex and the hippocampus in the rat. Behavioral Neuroscience, 116(5), 874-883.

Chipman, K., \& Kimura, D. (1998). An investigation of sex differences on incidental memory for verbal and pictorial material. Learning and Individual Differences, 10(4), 259-272.

Cimadevilla, J. M., Gonzalez-Pardo, H., Lopez, L., Diaz, F., Cueto, E. G., GarciaMoreno, L. M., \& Arias, J. L. (1999). Sex-related differences in spatial learning during the early postnatal development of the rat. Behavioural Processes, 46 , 159-171.

Clayton, N. S., \& Krebs, J. R. (1994). Hippocampal growth and attrition in birds affected by experience. Proceedings of the National Academy of Science USA, $91,7410-7414$.

Collaer, M. L., Geffner, M. E., Kaufman, F. R., Buckingham, B., \& Hines, M. (2002). Cognitive and behavioral characteristics of Turner syndrome: exploring a role for ovarian hormones in female sexual differentiation. Hormones and Behavior, 41, 139-155.

Connor, J. M., Schackman, M., \& Serbin, L. A. (1978). Sex-related differences in response to practice on a visual-spatial test and generalization to a related test. Child Development, 49, 24-29.

Conrad, C. D., Galea, L. A. M., Kuroda, Y., \& McEwen, B. S. (1996). Chronic stress impairs rat spatial memory on the $Y$ maze, and this effect is blocked by tianeptine pretreatment. Behavioral Neuroscience, 110(6), 1321-1334.

Cooper, B. G., \& Mizumori, S. J. Y. (2001). Temporary inactivation of the retrosplenial cortex causes a transient reorganisation of spatial coding in the hippocampus. The Journal of Neuroscience, 21(11), 3986-4001.

Corey, S. M. (1930). Sex differences in maze learning by white rats. Journal of Comparative Psychology, 10, 333-338.

Crawley, J., \& Goodwin, F. K. (1980). Preliminary report of a simple animal behavior model for the anxiolytic effects of benzodiaepines. Pharmacology Biochemistry and Behavior, 13, 167-170.

Critchlow, V., Liebelt, R. A., Bar-Sela, M., Mountcastle, W., \& Lipscomb, H. S. (1963). Sex difference in resting pituitary-adrenal function in the rat. American Journal of Physiology, 205, 807-815.

Dabbs, J. M. J., Chang, E.-L., Strong, R. A., \& Milun, R. (1998). Spatial ability, navigation strategy, and geographic knowledge among men and women. Evolution and Human Behavior, 19, 89-98.

Daniel, J. M., Fader, A. J., Spencer, A. L., \& Dohanich, G. P. (1997). Estrogen enhances performance of female rats during acquisition of a radial arm maze. Hormones and Behavior, 32, 217-225.

Daniel, J. M., Roberts, S. L., \& Dohanich, G. P. (1999). Effects of ovarian hormones and environment on radial maze and water maze performance of female rats. Physiology \& Behavior, 66(1), 11-20.

Davenport, J. W., Hagquist, W. W., \& Rankin, G. R. (1970). The symmetrical maze: an automated closed-field test series for rats. Behavior Research Methods and Instrumentation, 2(3), 112-119. 
Dawson, J. L. M., Cheung, Y. M., \&.Lau, R. T. S. (1973). Effects of neonatal sex hormones on sex-based cognitive abilities in the white rat. Psychologia, 16, 1724.

Dawson, J. L. M., Cheung, Y. M., \& Lau, R. T. S. (1975). Developmental effects of neonatal sex hormones on spatial and activity skills in the white rat. Biological Psychology, 3, 213-229.

de Araujo, I. E. T., Rolls, E. T., \& Stringer, S. M. (2001). A view model which accounts for the spatial fields of hippocampal primate spatial view cells and rat place cells. Hippocampus, 11(6), 699-706.

D'Hooge, R., \& De Deyn, P. P. (2001). Applications of the Morris water maze in the study of learning and memory. Brain Research Reviews, 36, 60-90.

Diamond, M. C. (1987). Sex differences in the rat forebrain. Brain Research Reviews, 12, 235-240.

Diamond, M. C. (1991). Hormonal effects on the development of cerebral lateralization. Psychoneuroendocrinology, 16(1-3), 121-129.

Dice, L. R., \& Howard, W. E. (1951). Distance of dispersal by prairie deermice from birthplaces to breeding sites. Contributions from the Laboratory of Vertebrate Biology, 50, 1-15.

Drickamer, L. C., \& Vessey, S. H. (1973). Group changing in free-ranging male rhesus monkeys. Primates, 14(4), 359-368.

Dringenberg, H. C., Richardson, D. P., Brien, J. F., \& Reynolds, J. N. (2001). Spatial learning in the guinea pig: cued versus non-cued learning, sex differences, and comparison with rats. Behavioural Brain Research, 124, 97-101.

Dudchenko, P. A., Goodridge, J. P., Seiterle, D. A., \& Taube, J. S. (1997). Effects of repeated disorientation on the acquisition of spatial tasks in rats: dissociation between the appetitive radial arm maze and aversive water maze. Journal of Experimental Psychology: Animal Behavior Processes, 23(2), 194-210.

Duff, S. J., \& Hampson, E. (2000). A beneficial effect of estrogen on working memory in postmenopausal women taking hormone replacement therapy. Hormones and Behavior, 38, 262-276.

Duff, S. J., \& Hampson, E. (2001). A sex difference on a novel spatial working memory task in humans. Brain and Cognition, 47, 470-493.

Durnin, J. V. G. A. (1991). Energy requirements of pregnancy. Diabetes, 40(Suppl. 2), 152-156.

Eals, M., \& Silverman, I. (1994). The hunter-gatherer theory of spatial sex differences: proximate factors mediating the female advantage in recall of object arrays. Ethology and Sociobiology, 15, 95-105.

Ebinger, P., \& Loehmer, R. (1987). A volumetric comparison of brains between greylag geese (Anser anser L.) and domestic geese. J. Hirnforsch, 28(3), 291299.

Einon, D. (1980). Spatial memory and response strategies in rats: age, sex and rearing differences in performance. Quarterly Journal of Experimental Psychology, 32, 473-489.

Ellard, C. G. (2000). Landmark navigation in gerbils: the role of the posterior parietal cortex. Psychobiology, 28(3), 325-338.

Endo, Y., T., M., Fujita, K., Funabashi, T., \& Kimura, F. (1994). Soft-diet feeding during development enhances later learning abilities in female rats. Physiology \& Behavior, 56(4), 629-633.

English, R. M., \& Hitchcock, N. E. (1968). Nutrient intakes during pregnancy, lactation and after the cessation of lactation in a group of Australian women. British Journal of Nutrition, 22, 615-624. 
Epting, L. K., \& Overman, W. H. (1998). Sex-sensitive tasks in men and women: a search for performance fluctuations across the menstrual cycle. Behavioral Neuroscience, 112(6), 1304-1317.

Fader, A. J., Johnson, P. E. M., \& Dohanich, G. P. (1999). Estrogen improves working but not reference memory and prevents amnestic effects of scopolamine on a radial-arm maze. Physiology Biochemistry and Behavior, 62(4), 711-717.

Faraday, M. M. (2002). Rat sex and strain differences in response to stress. Physiology \& Behavior, 75, 507-522.

Faul, F., \& Erdfelder, E. (1992). GPOWER: a priori, post-hoc, and compromise power analyses for MS-DOS (Version 2.0). Bonn: Bonn University, Dep. of Psychology.

Fernandes, C., Gonzalez, M. I., Wilson, C. A., \& File, S. E. (1999). Factor analysis shows that female rat behaviour is characterized primarily by activity, male rats are driven by sex and anxiety. Pharmacology Biochemistry and Behavior, 64(4), 731-738.

Fitch, R. H., \& Denenberg, V. H. (1998). A role for ovarian hormones in sexual differentiation of the brain. Behavioral and Brain Sciences, 21, 311-352.

Fitts, P. M. (1966). Cognitive aspects of information processing: III. Set for speed versus accuracy. Journal of Experimental Psychology, 71, 849-857.

Fitzgerald, R. E., Isler, R., Rosenberg, E., Oettinger, R., \& Baettig, K. (1985). Maze patrolling by rats with and without food reward. Animal Learning \& Behavior, 13(4), 451-462.

Foreman, N., \& Ermakova, I. (1998). The radial arm maze: twenty years on. In N. Foreman \& R. Gillett (Eds.), Handbook of Spatial Research Paradigms and Methodologies (Vol. 2 Clinical and Comparative Studies, pp. 87-278). Hove: Psychology Press.

Frick, K. M., \& Berger-Sweeney, J. (2001). Spatial reference memory and neocortical neurochemistry vary with the estrous cycle in C57BL/6 mice. Behavioral Neuroscience, 115(1), 229-237.

Frick, K. M., Burlingame, L. A., Arters, J. A., \& Berger-Sweeney, J. (2000). Reference memory, anxiety and estrous cyclicity in C57BL/6NIA mice are affected by age sex. Neuroscience, 95(1), 293-307.

Frye, C. A., Petralia, S. M., \& Rhodes, M. E. (2000). Estrous cycle and sex differences in performance on anxiety tasks coincide with increases in hippocampal progesterone and 3 alpha, 5 alpha-THP. Pharmacology, Biochemistry and Behavior, 67, 587-596.

Frye, C. A. (1994). Estrus-associated decrements in a water maze task are limited to acquisition. Physiology \& Behavior, 57(1), 5-14.

Galea, L. A. M., Kavaliers, M., \& Ossenkopp, K.-P. (1996). Sexually dimorphic spatial learning in meadow voles, Microtus pennsylvanicus, and deer mice, Peromyscus maniculatus. Journal of Experimental Biology, 199, 195-200.

Galea, L. A. M., Kavaliers, M., Ossenkopp, K.-P., \& Hampson, E. (1995). Gonadal hormone levels and spatial learning performance in the Morris water maze in male and female meadow voles, Microtus pennsylvanicus. Hormones and Behavior, 29, 106-125.

Galea, L. A. M., Kavaliers, M., Ossenkopp, K.-P., Innes, D., \& Hargreaves, E. L. (1994a). Sexually dimorphic spatial learning varies seasonally in two populations of deer mice. Brain Research, 635, 18-26.

Galea, L. A. M., \& Kimura, D. (1993). Sex differences in route-learning. Personality and Individual Differences, 14(1), 53-65. 
Galea, L. A. M., \& McEwen, B. S. (1999). Sex and seasonal differences in the rate of cell proliferation in the dendritic gyrus of adult wild meadow voles. Neuroscience, 89(3), 955-964.

Galea, L. A. M., Ormerod, B. K., Sampath, S., Kostaras, X., Wilkie, D. M., \& Phelps, M. T. (2000). Spatial working memory and hippocampal size across pregnancy in rats. Hormones and Behavior, 37, 86-95.

Galea, L. A. M., Ossenkopp, K.-P., \& Kavaliers, M. (1994b). Performance (reacquisition) of a water-maze task by adult meadow voles: effects of age of initial task acquisition and in utero environment (litter sex-ratio). Behavioural Brain Research, 63, 177-185.

Galea, L. A. M., Perrot-Sinal, T. S., Kavaliers, M., \& Ossenkopp, K.-P. (1999). Relations of hippocampal volume and dentate gyrus width to gonadal hormone levels in male and female meadow voles. Brain Research, 821, 383-391.

Galea, L. A. M., Saksida, L., Kavaliers, M., \& Ossenkopp, K.-P. (1994c). Naloxone facilitates spatial learning in a water-maze task in female, but not male, adult nonbreeding meadow voles. Pharmacology Biochemistry and Behavior, 47, 265271.

Gaulin, S. J. (1995). Does evolutionary theory predict sex differences in the brain? In M. S. Gazzaniga (Ed.), The Cognitive Neurosciences (pp. 1211-1224). Cambridge, Mass.: MIT Press.

Gaulin, S. J. C., \& Fitzgerald, R. W. (1986). Sex differences in spatial ability: an evolutionary hypothesis and test. The American Naturalist, 127(1), 74-88.

Gaulin, S. J. C., \& Fitzgerald, R. W. (1989). Sexual selection for spatial learning ability. Animal Behaviour, 37, 322-331.

Gaulin, S. J. C., Fitzgerald, R. W., \& Wartell, M. S. (1990). Sex differences in spatial ability and activity in two vole species (Microtus ochrogaster and $M$. pennsylvanicus). Journal of Comparative Psychology, 104(1), 88-93.

Gaulin, S. J. C., \& Hoffman, H. A. (1998). Evolution and development of sex differences in spatial ability. In L. Betzig, M. B. Mulder, \& P. Turke (Eds.), Human Reproductive Behaviour: a Danwinian Perspective. Cambridge: Cambridge University Press.

Gaulin, S. J. C., \& Wartell, M. S. (1990). Effects of experience and motivation on symmetrical-maze performance in the prarie vole (Microtus ochrogaster). Journal of Comparatve Psychology, 104(2), 183-189.

Geary, D. C. (1995). Sexual selection and sex differences in spatial cognition. Learning and Individual Differences, 7(4), 289-301.

Gibbs, M. E., \& Ng, K. T. (1977). Psychobiology of memory: towards a model of memory formation. Biobehavioral Reviews, 1, 113-136.

Gibbs, R. B. (2000). Long-term treatment with estrogen and progesterone enhances acquisition of a spatial memory task by ovariectomized aged rats. Neurobiology of Aging, 21, 107-116.

Gonzalez-Solis, J., Sokolov, E., \& Becker, P. H. (2001). Courtship feedings, copulations and paternity in common terns, Sterna hirundo. Animal Behaviour, $61,1125-1132$.

Good, M., Day, M., \& Muir, J. L. (1999). Cyclical changes in endogenous levels of oestrogen modulate the induction of LTD and LTP in the hippocampal CA1 region. European Journal of Neuroscience, 11, 4476-4480.

Gordon, H. W., \& Lee, P. L. (1993). No differences in cognitive performance between phases of the menstrual cycle. Psychoneuroendocrinology, 18(7), 521531. 
Gould, S. J., \& Lewontin, R. C. (1979). The spandrels of San Marco and the Panglossian paradigm: a critique of the adaptationist programme. Proceedings of the Royal Society of London B, 205, 581-598.

Gray, J. A., \& Buffery, A. W. H. (1971). Sex differences in emotional and cognitive behaviour in mammals including man: adaptive and neural bases. Acta Psychologica, 35, 89-111.

Green, M. W., Elliman, N. A., \& Rogers, P. J. (1997). Impaired cognitive processing in dieters: failure of attention focus or resource capacity limitation? British Journal of Health Psychology, 2, 259-267.

Green, M. W., \& Rogers, P. J. (1995). Impaired cognitive functioning during spontaneous dieting. Psychological Medicine, 25, 1003-1010.

Gron, G., Wunderlich, A. P., Spitzer, M., Tomczak, R., \& Riepe, M. W. (2000). Brain activation during human navigation: gender-different neural networks as substrate of performance. Nature Neuroscience, 3(4), 404-408.

Haga, Y. (1995). Effects of food deprivation and food reward on the behavior of rats in the radial-arm maze. Japanese Psychological Research, 37(4), 252-257.

Halpern, D. F. (1991). Sex Differences in Cognitive Abilities. (2nd ed.). Hillsdale, New Jersey: Lawrence Erlbaum Associates.

Halpern, D. F., \& Tan, U. (2001). Stereotypes and steroids: using a psychobiosocial model to understand cognitive sex differences. Brain and Cognition, 45, 392-414.

Hampson, E. (1990). Variations in sex-related cognitive-abilities across the menstrual-cycle. Brain and Cognition, 14, 26-43.

Hampson, E., \& Kimura, D. (1988). Reciprocal effects of hormonal fluctuations on human motor and perceptual-spatial skills. Behavioral Neuroscience, 102(3), 456-459.

Hampson, E., Rovet, J. F., \& Altmann, D. (1998). Spatial reasoning in children with cogenital adrenal hyperplasia due to 21 -hydroxylase deficiency. Developmental Neuropsychology, 14(2), 299-320.

Hampton, R. R., \& Shettleworth, S. J. (1996). Hippocampal lesions impair memory for location but not color in passerine birds. Behavioral Neuroscience, 110(4), 831-835.

Harker, K. T., \& Whishaw, I. Q. (2002). Impaired spatial performance in rats with retrosplenial lesions: importance of the spatial problem and the rat strain in identifying lesion effects in a swimming pool. Journal of Neuroscience, 22(3), $1155-1164$.

Hascoet, M., \& Bourin, M. (1998). A new approach to the light/dark test procedure in mice. Pharmacology Biochemistry and Behavior, 60(3), 645-653.

Hausmann, M., Slabbbekoorn, D., Van Goozen, S. H. M., Cohen-Kettenis, P. T., \& Gunturkun, O. (2000). Sex hormones affect spatial abilities during the menstrual cycle. Behavioral Neuroscience, 114(6), 1245-1250.

Hawkes, K. (1991). Showing off: tests of an hypothesis about men's foraging goals. Ethology and Sociobiology, 12, 29-54.

Healy, D. J., \& Drugan, R. C. (1996). Escapable stress modulates retention of spatial learning in rats: preliminary evidence for involvement of neurosteroids. Psychobiology, 24(2), 110-117.

Healy, S. D., Braham, S. R., \& Braithwaite, V. A. (1999). Spatial working memory in rats: no differences between the sexes. Proceedings of the Royal Society of London B, 266(1435), 2303-2308.

Healy, S. D., \& Cleland, J. A. (submitted). Food deprivation and spatial memory in coal tits (Parus ater) and great tits (Parus major). Behaviour. 
Healy, S. D., \& Krebs, J. R. (1992). Comparing spatial memory in two species of tit: recalling a single positive location. Animal Learning and Behavior, 20(2), 121126.

Henniger, M. S. H., Ohl, F., Holter, S. M., Weissenbacher, P., Toschi, N., Lorscher, P., Wigger, A., Spanagel, R., \& Landgraf, R. (2000). Unconditioned anxiety and social behaviour in two rat lines selectively bred for high and low anxiety-related behaviour. Behavioural Brain Research, 111, 153-163.

Hodges, H. (1996). Maze procedures: the radial-arm and water maze compared. Cognitive Brain Research, 3, 167-181.

Hull, E. M., Franz, J. R., Snyder, A. M., \& Nishita, J. K. (1980). Perinatal progesterone and learning, social and reproductive behavior in rats. Physiology \& Behavior, 24, 251-256.

Hurwitz, H. M. B., \& Davis, H. (1983). Depriving rats of food: a reappraisal of two techniques. Journal of the Experimental Analysis of Behavior, 40, 211-213.

Hyde, L. A., Hoplight, B. J., \& Denenberg, V. H. (1998). Water version of the radialarm maze: learning in three inbred strains of mice. Brain Research, 785, 236244.

laccino, J. F. (1993). Left Brain-Right Brain Differences: Inquiries, Evidence, and New Approaches. Hillsdale, New Jersey: Lawrence Erlbaum Associates, Inc.

Iqbal, S., Baziany, A., Hussain, M., James,.S., Wright, S., Hemmings, S., Shuaib, A., \& Rajput, A. (2002). Trimetazidine as a potential neuroprotectant in transient global ischemia in gerbils: a behavioral and histological study. Brain Research, 928, 1-7.

Isgor, C., \& Sengelaub, D. R. (1998). Prenatal gonadal steroids affect adult spatial behavior, CA1 and CA3 pyramidal cell morphology in rats. Hormones and Behavior, 34, 183-198.

Jacobs, L. F., Gaulin, S. J. C., Sherry, D. F., \& Hoffman, G. E. (1990). Evolution of spatial cognition: sex-specific patterns of spatial behavior predict hippocampal size. Proceedings of the National Academy of Science USA, 87, 6349-6352.

Jacobs, L. F., \& Spencer, W. D. (1994). Natural space-use patterns and hippocampal size in kangaroo rats. Brain, Behavior, and Evolution, 44, 125-132.

James, T. W., \& Kimura, D. (1997). Sex differences in remembering the locations of objects in an array: location-shifts versus location-exchanges. Evolution and Human Behavior, 18, 155-163.

Johnston, A. L., \& File, S. E. (1991). Sex differences in animal tests of anxiety. Physiology \& Behavior, 49, 245-250.

Joseph, R. (1979). Effects of rearing and sex on maze learning and competitive exploration in rats. The Journal of Psychology, 101, 37-43.

Joseph, R., Hess, S., \& Birecree, E. (1978). Effects of hormone manipulations and exploration on sex differences in maze learning. Behavioral Biology, 24, 364-377.

Juraska, J. M. (1991). Sex differences in 'cognitive' regions of the rat brain. Psychoneuroendocrinology, 16(1-3), 105-119.

Juraska, J. M., Henderson, C., \& Muller, J. (1984). Differential rearing experience, gender and radial maze performance. Developmental Psychobiology, 17(3), 209215.

Kanit, L., Taskiran, D., Furedy, J. J., Kulali, B., McDonald, R., \& Pogun, S. (1998a). Nicotine interacts with sex in affecting rat choices between 'look-out' and 'navigational' cognitive styles in the Morris water maze place learning task. Brain Research Bulletin, 46(5), 441-445.

Kanit, L., Taskiran, D., Yilmaz, O. A., Balkan, B., Demirgoren, S., Furedy, J. J., \& Pogun, S. (2000). Sexually dimorphic cognitive style in rats emerges after puberty. Brain Research Bulletin, 52(4), 243-248. 
Kanit, L., Yilmaz, O., Taskiran, D., Balkan, B., Furedy, J. J., \& Pogun, S. (1998b). Intersession interval affects performance in the Morris water maze. International Journal of Neuroscience, 96, 197-204.

Kant, J., Mougey, E. H., \& Meyerhoff, J. L. (1986). Diurnal variation in neuroendocrine response to stress in rats: plasma $A C T H$, beta-endorphin, beta$\mathrm{LPH}$, corticosterone, prolactin and pituitary AMP responses. Neuroendocrinology, 43, 383-390.

Kaplan, J. R., Fontenot, M. B., Berard, J., Manuck, S. B., \& Mann, J. J. (1995). Delayed dispersal and elevated monoaminergic activity in free-ranging Rhesus monkeys. American Journal of Primatology, 35, 299-234.

Katoh, A., Ishibashi, C., Shiomi, T., Takahara, Y., \& Eigyo, M. (1992). Ischemiainduced irreversible deficit of memory function in gerbils. Brain Research, 577, 57-63.

Kavaliers, M., Ossenkopp, K.-P., Galea, L. A. M., \& Kolb, B. (1998). Sex differences in spatial learning and prefrontal and parietal cortical dendritic morphology in the meadow vole, Microtus pennsylvanicus. Brain Research, 810, 41-47.

Kavaliers, M., Ossenkopp, K.-P., Prato, F. S., Innes, D. G. L., Galea, L. A. M., Kinsella, D. M., \& Perrot-Sinal, T. S. (1996). Spatial learning in deer mice: sex differences and the effects of endogenous opioids and $60 \mathrm{~Hz}$ magnetic fields. Journal of Comparative Physiology A, 179, 715-724.

Kesner, R. P., \& Long, J. M. (1998). Parietal cortex and a spatial cognitive map. Psychology, 26(2), 162-166.

Kessels, R. P. C., Postma, A., Wijnalda, E. M., \& de Haan, E. H. F. (2000). Frontallobe involvement in spatial memory: evidence from PET, fMRI, and lesion studies. Neuropsychology Review, 10(2), 101-113.

Kimble, D., \& Whishaw, I. Q. (1994). Spatial behavior in the Brazilian short-tailed opossum (Monodelphis domestica): comparison with the Norway rat (Rattus norvegicus) in the Morris water maze and radial arm maze. Journal of Comparative Psychology, 108(2), 148-155.

Kimura, D. (1999). Sex and Cognition. Cambridge, Massachusetts: MIT Press.

Kimura, D., \& Hampson, E. (1994). Cognitive pattern in men and women is influenced by fluctuations in sex hormones. Current Directions in Psychological Science, 3, 57-61.

King, J. A. (1983). Seasonal dispersal in a seminatural population of Peromyscus maniculatus. Canadian Journal of Zoology, 61, 2740-2750.

Koenig, W. D. (1989). Sex-biased dispersal in the contemporary United States. Ethology and Sociobiology, 10, 263-278.

Kolb, B., Buhrmann, K., McDonald, R., \& Sutherland, R. J. (1994). Dissociation of the medial prefrontal, posterior parietal, and posterior temporal cortex for spatial navigation and recognition memory in the rat. Cerebral Cortex, 4(6), 664-680.

Kolb, B., \& Cioe, J. (1996). Sex-related differences in cortical function after medial frontal lesions in rats. Behavioral Neuroscience, 110(6), 1271-1281.

Kolb, B., \& Stewart, J. (1991). Sex-related differences in dendritic branching of cells in the prefrontal cortex of rats. Journal of Neuroendocrinology, 3(1), 95-99.

Kondo, Y., Suzuki, K., \& Sakuma, Y. (1997). Estrogen alleviates cognitive dysfunction following transient brain ischemia in ovariectomized gerbils. Neuroscience Letters, 238, 45-48.

Koolhaas, J. M. (1999). The laboratory rat. In T. Poole (Ed.), UFAW Handbook on the Care and Management of Laboratory Animals. Volume 1: Terrestrial Vertebrates (7th ed., Vol. 1, pp. 313-330). Oxford: Blackwell Science.

Krasnoff, A., \& Weston, L. M. (1976). Puberal status and sex differences: activity and maze behavior in rats. Developmental Psychobiology, 9(3), 261-269. 
Kritzer, M. F., \& Kohama, S. G. (1998). Ovarian hormones influence the morphology, distribution, and density of tyrosine hydrolase immunoreactive axons in the dorsolateral prefrontal cortex of adult rhesus monkeys. Journal of Comparative Neurology, 395, 1-17.

Kritzer, M. F., McLaughlin, P. J., Smirlis, T., \& Robinson, J. K. (2001). Gonadectomy impairs t-maze acquisition in adult male rats. Hormones and Behavior, 39, 167174.

Kruska, D., \& Roehrs, M. (1974). Comparative-quantitative investigations on brains of feral pigs from the Galapagos islands and of European domestic pigs. Z. Anat. Entwickl.-Gesch., 144, 61-73.

LaBuda, C. J., Mellgren, R. L., \& Hale, R. L. (2002). Sex differences in the acquisition of a radial maze task in the CD- 1 mouse. Physiology \& Behavior, 76(2), 213-217.

Lachman, R., Lachman, J. L., \& Butterfield, E. C. (1979). Cognitive Psychology and Information Processing: An Introduction. Hillsdale, New Jersey: Lawrence Erlbaum Associates.

Lacreuse, A., Herndon, J. G., Killiany, R. J., Rosene, D. L., \& Moss, M. B. (1999). Spatial cognition in Rhesus monkeys: male superiority declines with age. Hormones and Behavior, 36, 70-76.

Lacreuse, A., Verreault, M., \& Herndon, J. G. (2001). Fluctuations in spatial recognition memory across the menstrual cycle in female rhesus monkeys. Psychoneuroendocrinology, 26(6), 623-639.

Lacreuse, A., Wilson, M. E., \& Herndon, J. G. (2002). Estradiol, but not raloxifene, improves aspects of spatial working memory in aged ovariectomized rhesus monkeys. Neurobiology of Aging, 23, 589-600.

Langley, C. M. (1994). Spatial memory in the desert kangaroo rat (Dipodomys deserti). Journal of Comparative Psychology, 108(1), 2-14.

Lawton, C. A. (1994). Gender differences in way-finding strategies: relationship to spatial ability and spatial anxiety. Sex Roles, 30(11/12), 765-779.

Lehmann, O., Bertrand, F., Jeltsch, H., Morer, M., Lazarus, C., Will, B., \& Cassel, J.C. (2002). 5,7-DHT-induced hippocampal 5-HT depletion attenuates behavioural deficits produced by $192 \mathrm{lg}$ G-saporin lesions of septal cholinergic neurons in the rat. European Journal of Neuroscience, 15, 1991-2006.

Levy, J. (1969). Possible bias for the evolution of lateral specialization of the human brain. Nature, 224, 614-615.

Li, A.-J., Oomura, Y., Sasaki, K., Suzuki, K., \& Hori, T. (1999). Protective effect of acidic fibroblast growth factor against ischemia-induced learning and memory deficits in two tasks in gerbils. Physiology \& Behavior, 66(4), 577-583.

Liang, S.-P., Kanthan, R., Shuaib, A., \& Wishart, T. (1997). Effects of clomethiazole on radial-arm maze performance following global forebrain ischemia in gerbils. Brain Research, 751, 189-195.

Linn, M. C., \& Petersen, A. C. (1985). Emergence and characterization of sex differences in spatial ability: a meta-analysis. Child Development, 56, 1479-1498.

Loskota, W. J., Lomax, P., \& Verity, M. A. (1974). A Stereotaxic Atlas of the Mongolian Gerbil Brain (Meriones unguiculatus). Ann Arbor, Michigan: Ann Arbor Science.

Lovejoy, C. O. (1981). The origin of man. Science, 211, 341-350.

Loy, R. (1986). Sexual dimorphism in the septohippocampal system. In R. Isaacson \& K. Pribram (Eds.), The Hippocampus Volume 3 (pp. 301-321). New York: Plenum. 
Luine, V., \& Rodriguez, M. (1994). Effects of estradiol on radial arm maze performance of young and aged rats. Behavioral and Neural Biology, 62, 230236.

Luine, V., Villegas, M., Martinez, C., \& McEwen, B. S. (1994). Repeated stress causes reversible impairments of spatial memory performance. Brain Research, $639,167-170$.

Lupien, S. J., \& McEwan, B. S. (1997). The acute effects of corticosteriods on cognition: integration of animal and human model studies. Brain Research Reviews, 24, 1-27.

Macdonald, D. W., \& Fenn, M. G. P. (1995). Rat ranges in arable areas. Journal of Zoology, 236(2), 349-353.

Machin, P., Vann, S. D., Muir, J. L., \& Aggleton, J. P. (2002). Neurotoxic lesions of the rat perirhinal cortex fail to disrupt the acquisition or performance of testes of allocentric spatial memory. Behavioral Neuroscience, 116(2), 232-240.

Madeira, M. D., Sousa, N., \& Paula-Barbosa, M. M. (1991). Sexual dimorphism in the mossy fiber synapses of the rat hippocampus. Experimental Brain Research, $87,537-545$.

Magalhaes, H. M., \& Carlini, E. L. A. (1974). Effects of perinatal testosterone treatment on body-weight, open field behavior and Lashley III maze performance of rats. Acta Physiol. Latinoam., 24, 317-327.

Maguire, E. A., Gadian, D. G., Johnsrude, I. S., Good, C. D., Ashburner, J., Frackowiak, R. S. J., \& Frith, C. D. (2000). Navigation-related structural change in the hippocampi of taxi drivers. Proceedings of the National Academy of Sciences USA, 97(8), 4398-4403.

Maier, D. M., \& Pohorecky, L. A. (1986). The effect of ethanol and sex on radial arm maze performance in rats. Pharmacology Biochemistry and Behavior, 25, 703709.

Manser, C. E. (1992). The Assessment of Stress in Laboratory Animals: R.S.P.C.A.

Marcondes, F. K., Miguel, K. J., Melo, L. L., \& Spadari-Bratfisch, R. C. (2001). Estrous cycle influences the response of female rats in the elevated plus-maze test. Physiology \& Behavior, 74, 435-440.

Markowska, A. L. (1999). Sex dimorphisms in the rate of age-related decline in spatial memory: relevance to alterations in the estrous cycle. The Journal of Neuroscience, 19(18), 8122-8133.

McBurney, D. H., Gaulin, S. J. C., Devineni, T., \& Adams, C. (1997). Superior spatial memory of women: stronger evidence for the gathering hypothesis. Evolution and Human Behavior, 18(3), 165-174.

McCormick, C. M., \& Teillon, S. M. (2001). Menstrual cycle variation in spatial ability: relation to salivary cortisol levels. Hormones and Behavior, 39, 29-38.

McDaniel, W. F., Williams, L. B., Attaway, C. M., \& Compton, D. M. (1998). Turnsignal utilization by rats with either unilateral or bilateral posterior parietal cortex injuries. Psychobiology, 26(2), 143-152.

McEwan, B. S., \& Sapolsky, R. M. (1995). Stress and cognitive function. Current Opinions in Neurobiology, 5, 205-216.

Mead, L. A., Hargreaves, E. L., Ossenkopp, K.-P., \& Kavaliers, M. (1995). A multivariate assessment of spontaneous locomotor activity in the Mongolian gerbil (Meriones unguiculatus): influences of ages and sex. Physiology \& Behavior, 57(5), 893-899.

Mealey, L. (2000). Sex Differences: Developmental and Evolutionary Strategies. San Diego: Academic Press. 
Means, L. W., Alexander, S. R., \& O'Neal, M. F. (1992). Those cheating rats: male and female rats use odor trails in a water-escape 'working memory' task. Behavioral and Neural Biology, 58, 144-151.

Mendl, M. (1999). Performing under pressure: stress and cognitive function. Applied Animal Behaviour Science, 65, 221-244.

Meyer, D. R. (1951). Food deprivation and discrimination reversal learning by monkeys. Journal of Experimental Psychology, 41, 10-16.

Miller, K. A., \& Dess, N. K. (1996). Dissociation of stress and food-deprivation effects on spatial performance. Psychobiology, 24(1), 38-43.

Mishima, N., Higashitani, F., Teraoka, K., \& Yoshioka, R. (1986). Sex differences in appetitive learning of mice. Physiology and Behavior, 37, 263-268.

Mitchell, A. S., Dalrymple-Alford, J. C., \& Christie, M. A. (2002). Spatial working memory and the brainstem cholinergic innervation to the anterior thalamus. Journal of Neuroscience, 22(5), 1922-1928.

Moeller, H., Goecke, B., \& Herter, F. (1988). Seasonal and diurnal changes of prostatic androgen receptor and circulating testosterone in young mature rats. Research in Experimental Medicine, 188, 451-462.

Moffat, S. D., \& Hampson, E. (1996). A curvilinear relationship between testosterone and spatial cognition in humans: possible influence of hand preference. Psychoneuroendocrinology, 21, 323-337.

Moffat, S. D., Hampson, E., \& Hatzipantelis, M. (1998). Navigation in a 'virtual' maze: sex differences and correlation with psychometric measures of spatial ability in humans. Evolution and Human Behavior, 19, 73-87.

Montello, D. R., Lovelace, K. L., Golledge, R. G., \& Self, C. M. (1999). Sex-related differences and similarities in geographic and environmental spatial abilities. Annals of the Association of American Geographers, 80(3), 515-534.

Moody, M. S. (1997). Changes in test scores on the mental rotations test during the menstrual cycle. Perceptual and Motor Skills, 84, 955-961.

Morris, R. G. M., Garrud, P., Rawlins, J. N. P., \& O'Keefe, J. (1982). Place navigation impaired in rats with hippocampal lesions. Nature, 297, 681-683.

Moser, M.-B., Trommald, M., \& Anderson, P. (1994). An increase in dendritic spine density on hippocampal CA1 pyramidal cells following spatial learning in adult rats suggests the formation of new synapses. Proceedings of the National Academy of Science USA, 91, 12673-12675.

Muir, G. M., \& Bilkey, D. K. (2001). Instability in the place field location of hippocampal place cells after lesions centred on the perirhinal cortex. The Journal of Neuroscience, 21(11), 4016-4025.

Mumenthaler, M. S., O'Hara, R., Taylor, J. L., Friedman, L., \& Yesavage, J. A. (2001). Relationship between variations in estradiol and progesterone levels across the menstrual cycle and human performance. Psychopharmacology, 155, 198-203.

Neave, N., Managed, M., \& Weightman, D. R. (1999). Sex differences in cognition: the role of testosterone and sexual orientation. Brain and Cognition, 41, 245-262.

Nilsson, M., Perfilieva, E., Johansson, U., Orwar, O., \& Eriksson, P. S. (1999). Enriched environment increases neurogenesis in the adult rat dentate gyrus and improves spatial memory. Journal of Neurobiology, 39, 569-578.

Nolte, J. (1999). The Human Brain: an Introduction to its Functional Anatomy. (4th ed.). St. Louis: Mosby.

Norris, D. O. (1997). Vertebrate Endocrinology. (3rd ed.). San Diego: Academic Press. 
Norris, M. L. (1987). Gerbils. In T. B. Poole (Ed.), The UFAW handbook on the care and management of laboratory animals (6th ed.). Harlow: Longman Scientific \& Technical.

Nyborg, H. (1983). Spatial ability in men and women: review and new theory. Advances in Behaviour Research and Therapy, 5(2), 89-140.

O'Keefe, J., \& Dostrovsky, J. (1971). The hippocampus as a spatial map. Preliminary evidence from unit activity in the freely moving rat. Brain Research, 34, 171-175.

O'Keefe, J., \& Nadel, L. (1978). The Hippocampus as a Cognitive Map. Oxford: Oxford University Press.

Olson, D. M., Eliot, J., \& Hardy, R. C. (1988). Relationships between activities and sex-related differences in performance on spatial tests. Perceptual and Motor Skills, 67, 223-232.

Olthof, A., Sutton, J. E., Slumskie, S. V., D'Addetta, J., \& Roberts, W. (1999). In search of the cognitive map: can rats learn an abstract pattern of rewarded arms on the radial maze? Journal of Experimental Psychology, 25(3), 352-362.

Olton, D. S., \& Samuelson, R. J. (1976). Remembrance of places passed: spatial memory in rats. Journal of Experimental Psychology: Animal Behavior Processes, 2(2), 97-116.

Papi, F., \& Wallraff, H. G. (1992). Birds. In F. Papi (Ed.), Animal Homing (pp. 263319). London: Chapman \& Hall.

Pare, W. P. (1965). Stress and consummatory behavior in the albino rat. Psychological Reports, 16, 399-405.

Paxinos, G., \& Watson, C. (1997). The rat brain in stereotaxic coordinates. (Compact 3rd ed.). San Diego: Academic Press.

Pearce, J. M., Roberts, A. D. L., \& Good, M. (1998). Hippocampal lesions disrupt navigation based on cognitive maps but not heading vectors. Nature, 396, 75-77.

Perrot-Sinal, T. S., Kostenuik, M. A., Ossenkopp, K.-P., \& Kavaliers, M. (1996). Sex differences in performance in the Morris water maze and the effects of initial nonstationary hidden platform training. Behavioral Neuroscience, 110(6), 13091320.

Petersen, K., \& Sherry, D. F. (1996). No sex difference occurs in hippocampus, food-storing, or memory for food caches in black-capped chickadees. Behavioural Brain Research, 79, 15-22.

Phillips, K., \& Silverman, I. (1997). Differences in the relationship of menstrual cycle phase to spatial performance on two- and three-dimensional tasks. Hormones and Behavior, 32, 167-175.

Pope, J. F., Skinner, J. D., \& Carruth, B. R. (1992). Cravings and aversions of pregnant adolescents. Journal of the American Dietetic Association, 92(12), 1479-1482.

Postma, A., Izendoorn, R., \& De Haan, E. H. F. (1998). Sex differences in object location memory. Brain and Cognition, 36, 334-345.

Postma, A., Meyer, G., Tuiten, A., van Honk, J., Kessels, R. P. C., \& Thijssen, J. (2000). Effects of testosterone administration on selective aspects of objectlocation memory in healthy young women. Psychoneuroendocrinology, 25, 563575.

Postma, A., Winkel, J., Tuiten, A., \& van Honk, J. (1999). Sex differences and menstrual cycle effects in human spatial memory. Psychoneuroendocrinology, 24, 175-192.

Poucet, B., \& Benhamou, S. (1997). The neuropsychology of spatial cognition in the rat. Critical reviews in neurobiology, 11(2\&3), 101-120. 
Prentice, A. M., Spaaij, C. J. K., Goldberg, G. R., Poppitt, S. D., Van Raaij, J. M. A., Totton, M., Swann, D., \& Black, A. E. (1996). Energy requirements of pregnant and lactating women. European Journal of Clinical Neuroscience, 50(Suppl. 1), S82-S111.

Ramos, A., Berton, O., Mormede, P., \& Chaoloff, F. (1997). A multiple-test study of anxiety-related behaviours in six inbred rat strains. Behavioural Brain Research, 85, 57-69.

Reboreda, J. C., Clayton, N. S., \& Kacelnik, A. (1996). Species and sex differences in hippocampus size in parasitic and non-parasitic cowbirds. NeuroReport, 7, 505-508.

Rehkämper, G., Haase, E., \& Frahm, H. D. (1988). Allometric comparison of brain weight and brain structure volumes in different breeds of domestic pigeon, Columba livia f.d. (fantails, homing pigeons, strassers). Brain Behaviour and Evolution, 31, 141-149.

Roof, R. L. (1993a). The dentate gyrus is sexually dimorphic in prepubescent rats: testosterone plays a significant role. Brain Research, 610, 148-151.

Roof, R. L. (1993b). Neonatal exogenous testosterone modifies sex difference in radial arm and Morris water maze performance in prepubescent and adult rats. Behavioural Brain Research, 53, 1-10.

Roof, R. L., \& Havens, M. D. (1992). Testosterone improves maze performance and induces development of a male hippocampus in females. Brain Research, 572, 310-313.

Roof, R. L., \& Stein, D. G. (1999). Gender differences in Morris water maze performance depends on task parameters. Physiology and Behavior, 68, 81-86.

Roof, R. L., Zhang, Q., Glasier, M. M., \& Stein, D. G. (1993). Gender-specific impairment on Morris water maze task after entorhinal cortex lesion. Behavioural Brain Research, 57, 47-51.

Rosenzweig, M. R., Breedlove, S. M., \& Leiman, A. L. (2002). Biological Psychology. (3rd ed.). Sunderland, Massachusetts: Sinauer Associates, Inc.

Rosenzweig, M. R., \& Leiman, A. L. (1982). Physiological Psychology. Lexington, Massachusetts: D. C. Heath \& Company.

Samuels, M. L. (1989). Statistics for the Life Sciences. Englewood Cliffs, New Jersey: Prentice-Hall.

Sandstrom, N. J., \& Williams, C. L. (2001). Memory retention is modulated by acute estradiol and progesterone replacement. Behavioral Neuroscience, 115(2), 384393.

Saucier, D. M., Green, S. M., Leason, J., MacFadden, A., Bell, S., \& Elias, L. J. (2002). Are sex differences in navigation caused by sexually dimorphic strategies or by differences in the ability to use the strategies? Behavioral Neuroscience, $116(3), 403-410$.

Save, E., \& Poucet, B. (2000). Involvement of the hippocampus and associative parietal cortex in the use of proximal and distal landmarks for navigation. Behavioural Brain Research, 109, 195-206.

Save, E., Poucet, B., Foreman, N., \& Thinus-Blanc, C. (1998). The contribution of the associative parietal cortex and hippocampus to spatial processing in rodents. Psychology, 26(2), 153-161.

Schenk, F., Contant, B., \& Werffeli, P. (1990). Intrahippocampal cholinergic grafts in aged rats compensate impairments in a radial maze and in a place learning task. Experimental Brain Research, 82, 641-650.

Schwartz-Bloom, R., McDonough, K. J., Chase, P. J., Chadwick, L. E., Inglefield, J. R., \& Levin, E. D. (1998). Long-term neuroprotection by benzodiazepine: full 
versus partial agonists after transient cerebral ischemia in the gerbil. Journal of Cerebral Blood Flow and Metabolism, 18, 548-558.

Seymoure, P., Dou, H., \& Juraska, J. M. (1996). Sex differences in radial maze performance: influence of rearing environment and room cues. Psychobiology, 24(1), 33-37.

Sherman, J. A. (1967). Problem of sex differences in space perception and aspects of intellectual functioning. Psychological Review, 74, 290-299.

Sherry, D. F., Forbes, M. R. L., Khurgel, M., \& Ivy, G. O. (1993). Females have a larger hippocampus than males in the brood-parasitic brown-headed cowbird. Proceedings of the National Academy of Sciences USA, 90,7839-7843.

Sherry, D. F., Galef, B. G., \& Clark, M. M. (1996). Sex and intrauterine position influence the size of the gerbil hippocampus. Physiology and Behavior, 60(6), 1491-1494.

Sherry, D. F., \& Hampson, E. (1997). Evolution and the hormonal control of sexually-dimorphic spatial abilities in humans. Trends in Cognitive Sciences, 1(2), 50-56.

Sherry, D. F., Jacobs, L. F., \& Gaulin, S. J. C. (1992). Spatial memory and adaptive specialization of the hippocampus. TINS, 15(8), 298-303.

Sherry, D. F., \& Vaccarino, A. L. (1989). Hippocampus and memory for food caches in black-capped chickadees. Behavioral Neuroscience, 103(2), 308-318.

Shors, T. J. (1998). Stress and sex effects on associative learning: for better or for worse. The Neuroscientist, 4(5), 353-364.

Shors, T. J., \& Dryver, E. (1992). Stress impedes exploration and the acquisition of spatial information in the eight-arm radial maze. Psychobiology, 20(4), 247-253.

Shuaib, A., Waqaar, T., ljaz, M. S., Kanthan, R., Wishart, T., \& Howlett, W. (1996). Neuroprotection with felbamate: a 7-and 28-day study in transient forebrain ischemia in gerbils. Brain Research, 727, 65-70.

Silverman, I., Choi, J., Mackewn, A., Fisher, M., Moro, J., \& Olshansky, E. (2000). Evolved mechanisms underlying wayfinding: further studies on the huntergatherer theory of spatial sex differences. Evolution and Human Behavior, 21, 201-213.

Silverman, I., \& Eals, M. (1992). Sex differences in spatial abilities: evolutionary theory and data. In J. Barkow, L. Cosmides, \& J. Tooby (Eds.), The Adapted Mind: Evolutionary Psychology and the Generation of Culture (pp. 533-549). Oxford: Oxford University Press.

Silverman, I., Kastuk, D., Choi, J., \& Phillips, K. (1999). Testosterone levels and spatial ability in men. Psychoneuroendocrinology, 24, 813-822.

Silverman, I., Phillips, K., \& Silverman, L. K. (1996). Homogeneity of effect sizes for sex across spatial tests and cultures: implications for hormonal theories. Brain and Cognition, 31, 90-94.

Slob, A. K., \& van der Werff ten Bosch, J. J. (1975). Sex differences in body growth in the rat. Physiology \& Behavior, 14, 353-361.

Stackman, R. W., Blasberg, M. E., Langan, C. J., \& Clark, A. S. (1997). Stability of spatial working memory across the estrous cycle of Long-Evans rats. Neurobiology of Learning and Memory, 67, 167-171.

Stewart, C. A., \& Morris, R. G. M. (1993). The watermaze. In A. Sahgal (Ed.), Behavioural Neuroscience (Vol. 1 A Practical Approach, pp. 107-122). Oxford: Oxford University Press.

Stewart, J., Skvarenina, A., \& Pottier, J. (1975). Effects of neonatal androgens on open-field behavior and maze learning in the prepubescent and adult rat. Physiology and Behavior, 14, 291-295. 
Stillman, M. J., Shukitt-Hale, B., Levy, A., \& Lieberman, H. R. (1998). Spatial memory under acute cold and restraint stress. Physiology \& Behavior, 64(5), 605609.

Strasser, R., Bingman, V. P., loale, P., Casini, G., \& Bagnoli, P. (1998). The homing pigeon hippocampus and the development of landmark navigation. Developmental Psychobiology, 33, 305-315.

Swihart, R. K., \& Slade, N. A. (1989). Differences in home-range size between sexes of Microtus ochrogaster. Journal of Mammalogy, 70(4), 816-820.

Tabibnia, G., Cooke, B. M., \& Breedlove, S. M. (1999). Sex difference and laterality in the volume of mouse dentate gyrus granule cell layer. Brain Research, 827, 41-45.

Taylor, K. D. (1978). Range of movement and activity of common rats (Rattus norvegicus) on agricultural land. Journal of Applied Ecology, 15, 663-677.

Tees, R. C., Midgeley, G., \& Nesbit, J. C. (1981). The effect of early visual experience on spatial maze learning in rats. Developmental Psychobiology, 14(5), 425-438.

Telegdy, G. A., \& Cohen, J. S. (1971). Cue utilization and drive level in albino rats. Journal of Comparative and Physiological Psychology, 75(2), 248-253.

Tillé, Y., Newman, J. A., \& Healy, S. D. (1996). New tests for departures from random behavior in spatial memory experiments. Animal Learning \& Behavior, 24(3), 327-340.

Timberlake, W., \& White, W. (1990). Winning isn't everything: rats need only food deprivation and not food reward to efficiently traverse a radial arm maze. Learning and Motivation, 21, 153-163.

Toth, L. A., \& Gardiner, T. W. (2000). Food and water restriction protocols: physiological and behavioral considerations. Contemporary Topics in Laboratory Animal Science, 39(6), 9-17.

Tropp, J., \& Markus, E. J. (2001a). Effects of mild food deprivation on the estrous cycle of rats. Physiology \& Behavior, 73, 553-559.

Tropp, J., \& Markus, E. J. (2001b). Sex differences in the dynamics of cue utilization and exploratory behavior. Behavioural Brain Research, 119, 143-154.

Van Goozen, S. H. M., Cohen-Kettenis, P. T., Gooren, L. J. G., Frijda, N. H., \& Van de Poll, N. E. (1995). Gender differences in behaviour: activating effects of crosssex hormones. Psychoneuroendocrinology, 20(4), 343-363.

Van Haaren, F., Wouters, M., \& Van de Poll, N. (1987). Absence of behavioral differences between male and female rats in different radial-maze procedures. Physiology \& Behavior, 39, 409-412.

Van Hest, A., Van Haaren, F., \& Van de Poll, N. (1988). The behavior of male and female Wistar rats pressing a lever for food is not affected by sex differences in food motivation. Behavioural Brain Research, 27, 215-221.

Vandenbogaerde, A., Zanoli, P., Puia, G., Truzzi, C., Kamuhabwa, A., De Witte, P., Merlevede, W., \& Baraldi, M. (2000). Evidence that total extract of Hypericum perforatum affects exploratory behavior and exerts anxiolytic effects in rats. Pharmacology Biochemistry and Behavior, 65(4), 627-633.

Vawter, M. P., \& Van Ree, J. M. (1989). Hole board food search task in rats: effects of hole depths and food deprivation. Physiology \& Behavior, 45, 891-895.

Viau, V., \& Meaney, M. J. (1991). Variations in the hypothalamic-pituitary-adrenal response to stress during the estrous cycle in the rat. Endocrinology, 129(5), 2503-2511.

Von Lubnitz, D. K. J. E., Beenhakker, M., Lin, R. C.-S., Carter, M. F., Paul, I. A., Bischofberger, N., \& Jacobson, K. A. (1996). Reduction of postischemic brain 
damage and memory deficits following treatment with the selective adenosine $A_{1}$ receptor agonist. European Journal of Pharmacology, 302, 43-48.

Wade, S. E., \& Maier, S. F. (1986). Effects of individual housing and stressor exposure upon acquisition of watermaze escape. Learning and Motivation, 17, 287-310.

Warren, S. G., Humpreys, A. G., Juraska, J. M., \& Greenough, W. T. (1995). LTP varies across the estrous cycle: enhanced synaptic plasticity in proestrus rats. Brain Research, 703, 26-30.

Warren, S. G., \& Juraska, J. M. (1997). Spatial and nonspatial learning across the rat estrous cycle. Behavioral Neuroscience, 111(2), 259-266.

Watanabe, Y., Gould, E., \& McEwan, B. S. (1992). Stress induces atrophy of apical dendrite of hippocampal CA3 pyramidal neurons. Brain Research, 588, 341-345.

Watson, N. V., \& Kimura, D. (1991). Nontrivial sex differences in throwing and intercepting: relation to psychometrically-defined spatial functions. Personality and Individual Differences, 12(5), 375-385.

Waynforth, H. B., \& Flecknell, P. A. (1992). Experimental and Surgical Technique in the Rat. (2 ed.). London: Academic Press.

Weinstock, R. B. (1972). Maintenance schedules and hunger drive: an examination of the rat literature. Psychological Bulletin, 78(4), 311-320.

Whishaw, I. Q., \& Tomie, J.-A. (1996). Of mice and mazes: similarities between mice and rats on dry land but not water mazes. Physiology \& Behavior, 60(5), 1191-1197.

Wilkie, D. M., Mumby, D. G., Needham, G., \& Smeele, M. (1992). Sustained arm visiting by nondeprived, nonrewarded rats in a radial maze. Bulletin of the Psychonomic Society, 30(4), 314-316.

Wilkie, D. M., \& Slobin, P. (1983). Gerbils in space: performance on the 17-arm radial maze. Journal of the Experimental Analysis of Behavior, 40, 301-312.

Williams, C. L., Barnett, A. M., \& Meck, W. H. (1990). Organizational effects of early gonadal secretions on sexual differentiation in spatial memory. Behavioral Neuroscience, 104(1), 84-97.

Williams, C. L., \& Meck, W. H. (1991). The organizational effects of gonadal steroids on sexually dimorphic spatial ability. Psychoneuroendocrinology, 16(1-3), 155176.

Wood, G. E., \& Shors, T. J. (1998). Stress facilitates classical conditioning in males, but impairs classical conditioning in females through activational effects of ovarian hormones. Proceedings of the National Academy of Science USA, 95, 4066-4071.

Woodfield, R. L. (1984). Embedded figures test performance before and after childbirth. British Journal of Psychology, 75, 81-88.

Woolley, C. S., Gould, E., Frankfurt, M., \& McEwan, B. S. (1990a). Naturally occurring fluctuations in dendritic spine density on adult hippocampal pyramidal neurons. Journal of Neuroscience, 10(12), 4035-4039.

Woolley, C. S., Gould, E., \& McEwan, B. S. (1990b). Exposure to excess glucocorticoids alters dendritic morphology of adult hippocampal pyramidal neurons. Brain Research, 531, 225-231.

Wynn, T. G., Tierson, F. D., \& Palmer, C. T. (1996). Evolution of sex differences in spatial cognition. Yearbook of Physical Anthropology, 39, 11-42.

Zaretsky, H. H. (1966). Learning and performance in the runway as a function of the shift in drive and incentive. Journal of Comparative and Physiological

Psychology, 62(2), 218-221. 
Zhou, J., Zhang, H. Y., \& Tang, X. C. (2001). Huperzine A attenuates cognitive deficits and hippocampal neuronal damage after transient global ischemia in gerbils. Neuroscience Letters, 313, 137-140.

Zielinski, W. J., vom Saal, F. S., \& Vandenbergh, J. G. (1992). The effect of intrauterine position on the survival, reproduction and home range size of female house mice (Mus musculus). Behavioral Ecology and Sociobiology, 30, 185-191. 